\title{
Catalytic, Transition Metal-Free Semireduction of Propiolamide Derivatives: Scope and Mechanistic Investigation
}

\author{
R. Justin Grams, Christopher J. Garcia, Connor Szwetkowski, and Webster L. Santos* \\ Department of Chemistry, Virginia Tech, 900 West Campus Drive, Blacksburg, VA 24061 (USA) \\ *E-mail: santosw@vt.edu
}

Table of Contents

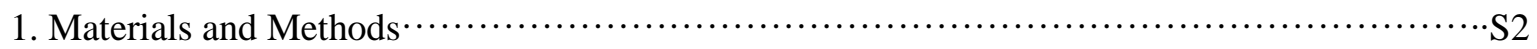

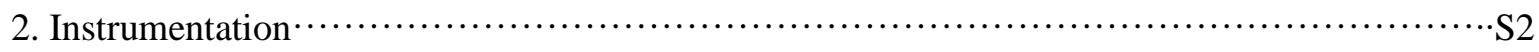

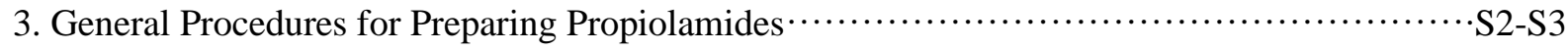

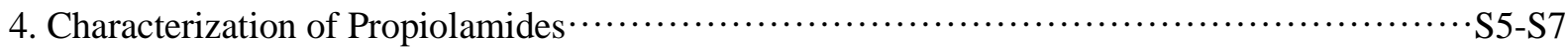

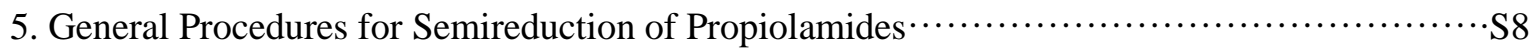

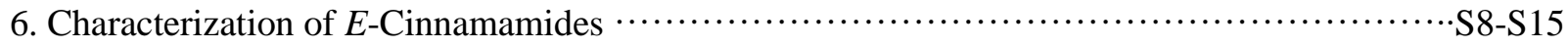

7. Synthesis and Characterization of FK866 Analog: Intermediates and Final E-Cinnamamide $\cdots$ S16-S18

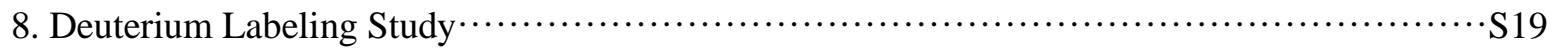

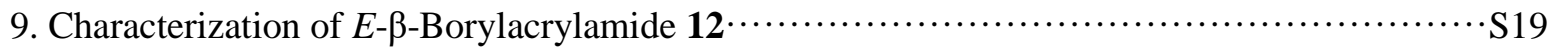

10. Procedures for Control Reactions with $\mathrm{BH}_{3}$ (Scheme $7 \mathrm{C}$ in the manuscript) $\cdots \cdots \cdots \cdots \cdots \cdots \cdots \cdot \mathrm{S} 20$

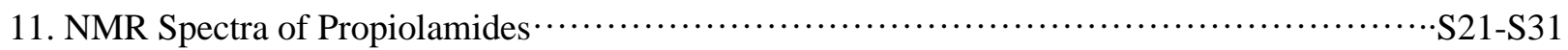

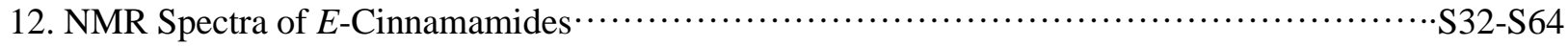

13. NMR Spectra of FK866 Analog: Intermediates and Final E-Cinnamamide $\cdots \cdots \cdots \cdots \cdots \cdots \cdots \cdots \cdot$ S65-S68

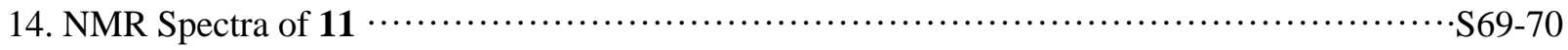

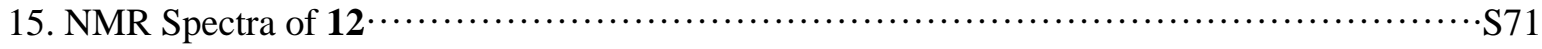

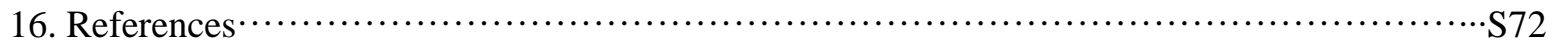




\section{Materials and Methods}

Reactions were performed using Schlenk technique under Argon or Nitrogen atmosphere. All glassware used was flame-dried or oven-dried overnight. Chemicals were obtained from commercial sources unless otherwise noted. THF and DCM were dried using the Innovative Technology Pure SolvMD solvent purification system. Column chromatography was performed using SiliaFlash P60 40-63 $\mu \mathrm{m}, 60 \AA$. TLC analyses were performed using Silicycle aluminum backed silica gel F-254 plates.

\section{Instrumentation}

NMR spectroscopic experiments were performed using an Agilent 400-MR $400 \mathrm{MHz}$, an Agilent U4-DD2 $400 \mathrm{~Hz}$, or a Bruker Avance II $500 \mathrm{MHz}$ spectrometer. Chemical shifts are reported in $\delta \mathrm{ppm}$ and ${ }^{1} \mathrm{H}$ and ${ }^{13} \mathrm{C}$ NMR are referenced to an internal standard $\left(\mathrm{CDCl}_{3}, \mathrm{CD}_{3} \mathrm{OD}\right.$, DMSO- $d_{6}$, TMS, or Acetone- $\left.d_{6}\right)$. Data are reported as follows: chemical shift, multiplicity $(\mathrm{s}=$ singlet, $\mathrm{d}=$ doublet, $\mathrm{t}=$ triplet, $\mathrm{q}=$ quartet, $\mathrm{dd}=$ doublet of doublets, $\mathrm{dt}=$ doublet of triplets, $\mathrm{ddd}=$ doublet of doublet of doublets, $\mathrm{m}=$ multiplet), coupling constants $(\mathrm{Hz})$, and integration. All propiolamides existed as a 9:1 mixture of rotamers and rotamer peaks are assigned an asterisk. In the case where a cinnamamide was isolated and contained rotamers, the rotamer peaks are assigned an asterisk. $E$ - or Z-isomers of cinnamamides are reported as major (E/trans-isomer) and minor (Z/cis-isomer). ESI mass spectra were acquired using an Agilent 6220 TOF LC-MS.

\section{General Procedures for Preparing Propiolamides}

Propiolamides 1a-c, 1e-k, 1m-o, 3a-b, 3d-e, 5a-c, and $\mathbf{5 f}$ were prepared in accordance with previous literature and references for the spectra of previously known propargylamides are provided in Table S1. Propiolamides 1d, 1l, 1p, 3c, 3f, 5d-e and 5g-i were prepared according to the following general procedures unless noted otherwise. All propiolamides existed as a 9:1 mixture of rotamers and rotamer peaks are assigned an asterisk.

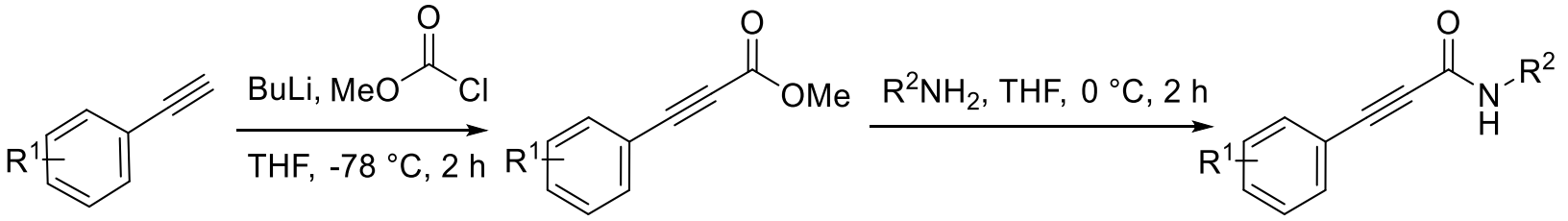

Procedure 3.1. To a flame- or oven-dried round bottom flask fixed with a stir bar was added dry THF (20 $\mathrm{mL}$ ) and the terminal acetylene $(5.0 \mathrm{mmol})$ under inert atmosphere and cooled to $-78{ }^{\circ} \mathrm{C}$ (dry ice/acetone bath). $n$-Butyllithium (5.0 mmol, $2.5 \mathrm{M}$ in hexanes), was then added dropwise to the mixture at $-78{ }^{\circ} \mathrm{C}$ for 15 minutes. Methyl chloroformate $(5.0 \mathrm{mmol})$ was then added dropwise to the mixture at $-78{ }^{\circ} \mathrm{C}$ and the reaction was stirred for 2 hours. Upon completion, the reaction was diluted with water and extracted with ethyl acetate. The organic layer was washed with brine and dried over anhydrous sodium sulfate. Concentration in vacuo yielded a yellow oil which upon purification by silica gel column chromatography (1:10, EtOAc:hexanes) yielded the methyl propiolate ester as a colorless oil. The methyl propiolate ester $(2.0 \mathrm{mmol})$ was added to a round-bottom flask fixed with a stir bar and diluted with THF $(2 \mathrm{~mL})$ which was then added dropwise to a solution of methylamine ( $8 \mathrm{M}$ in $\mathrm{EtOH}, 4.0 \mathrm{mmol}$ ) or ammonia ( $7 \mathrm{~N}$ in $\mathrm{MeOH}, 4.0$ $\mathrm{mmol}$ ) at $0{ }^{\circ} \mathrm{C}$ (brine/ice bath). After 2 hours, the reaction was diluted with water and extracted with ethyl acetate. The organic layer was washed with brine and dried over anhydrous sodium sulfate. Concentration in vacuo yielded the crude product as a yellow oil, which was then purified by silica gel column chromatography (eluted with a 2:3 ethyl acetate:hexanes solvent system) to afford the pure propiolamide as a white solid, which was dried by dissolving in toluene $(3 \mathrm{~mL}, 3 \mathrm{x})$ and concentrating the product in vacuo. 
Procedure 3.2. To an oven-dried $25 \mathrm{~mL}$ 2-neck round bottom flask with stir bar was fixed a $15 \mathrm{~mL}$ liquid addition funnel. To the round bottom flask was added potassium carbonate (14 mmol), $\mathrm{PdCl}_{2}\left(\mathrm{PPh}_{3}\right)_{3}(5 \mathrm{~mol}$ $\%$ ), and copper iodide (5 mol \%), which was then purged with $\mathrm{N}_{2}$ via Schlenk technique. 1,2dimethoxyethane (DME) and aryl iodide $(7.1 \mathrm{mmol})$ were then added, followed by an additional 20 minutes of purging the solution with $\mathrm{N}_{2}$. To the addition funnel was added propiolate ester (14 mmol) and DME (to make a $0.6 \mathrm{M}$ solution). The solution in the round bottom flask was heated to $70{ }^{\circ} \mathrm{C}$. Once heated, the propiolate ester solution was slowly added dropwise over the course of 1 hour. The product was purified by silica gel column chromatography (eluted with a 2:3 ethyl acetate in hexanes solvent system) yielding the propiolate as a clear oil. The propiolate was then subjected to amide forming conditions as described in Procedure 3.1.

Procedure 3.3. To a $15 \mathrm{~mL}$ round bottom flask fixed with a stir bar was added DCC $(0.99 \mathrm{mmol})$ and DMAP $(0.090 \mathrm{mmol})$ and dichloromethane $(0.90 \mathrm{~mL})$. The round bottom flask was submerged in an ice/brine bath and the solution was allowed to cool to $0{ }^{\circ} \mathrm{C}$ over 15 minutes. Propiolic acid ( $\left.0.90 \mathrm{mmol}\right)$ was diluted with dichloromethane $(1.6 \mathrm{~mL})$ and was added to the DCC/DMAP solution. The reaction was stirred at $0{ }^{\circ} \mathrm{C}$ for $c a .10$ minutes. Propargylamine $(0.99 \mathrm{mmol})$ was diluted with dichloromethane $(0.99 \mathrm{~mL})$ and added dropwise to the reaction mixture. The reaction was allowed to slowly warm to room temperature and was stirred overnight. The reaction mixture was diluted with ethyl acetate and filtered through a short bed of silica. The organic layer was washed with water and brine, then dried over sodium sulfate. The organic layer was concentrated in vacuo and purified by silica gel column chromatography (eluted with a 2:3 ethyl acetate in hexanes solvent system) to afford the pure propiolamide as a white solid, which was dried by dissolving in toluene $(3 \mathrm{~mL}, 3 \mathrm{x})$ and concentrating the product in vacuo. 
Table S1. References for Propargylamide Substrates

\begin{tabular}{|c|c|c|c|}
\hline Substrate & $\mathbf{R}^{1}$ & $\mathbf{R}^{2}$ & Reference \\
\hline 1a & $\mathrm{H}$ & $\mathrm{CH}_{3}$ & J. Org. Chem. $1985,50,3979$ \\
\hline 1b & $\mathrm{H}$ & $\mathrm{CH}\left(\mathrm{CH}_{3}\right)_{2}$ & $\begin{array}{l}\text { Angew. Chem. Int. Ed. 2017, 56, } \\
5111\end{array}$ \\
\hline 1c & $\mathrm{H}$ & $\mathrm{Ph}$ & $\begin{array}{l}\text { Angew. Chem. Int. Ed. 2017, 56, } \\
5111\end{array}$ \\
\hline $1 \mathrm{e}$ & $\mathrm{H}$ & $\mathrm{CH}_{2} \mathrm{CCH}$ & Tetrahedron, 2019, 75, 3586 \\
\hline 1f & $p-\mathrm{OCH}_{2} \mathrm{CH}_{3}$ & $\mathrm{CH}_{3}$ & $\begin{array}{l}\text { Angew. Chem. Int. Ed. 2017, 56, } \\
5111\end{array}$ \\
\hline $1 \mathrm{~g}$ & $o-\mathrm{OCH}_{3}$ & $\mathrm{CH}_{3}$ & $\begin{array}{l}\text { Angew. Chem. Int. Ed. 2017, 56, } \\
5111\end{array}$ \\
\hline 1h & $p$-OBn & $\mathrm{CH}_{3}$ & $\begin{array}{l}\text { Angew. Chem. Int. Ed. 2017, 56, } \\
5111\end{array}$ \\
\hline 1i & $m-\mathrm{CH}_{3}$ & $\mathrm{CH}_{3}$ & $\begin{array}{l}\text { Angew. Chem. Int. Ed. 2017, 56, } \\
5111\end{array}$ \\
\hline $\mathbf{1 j}$ & $p-\mathrm{C}\left(\mathrm{CH}_{3}\right)_{3}$ & $\mathrm{CH}_{3}$ & $\begin{array}{l}\text { Angew. Chem. Int. Ed. 2017, 56, } \\
5111\end{array}$ \\
\hline $1 \mathrm{k}$ & $o-\mathrm{Cl}$ & $\mathrm{CH}_{3}$ & $\begin{array}{l}\text { Angew. Chem. Int. Ed. 2017, 56, } \\
5111\end{array}$ \\
\hline $1 \mathrm{~m}$ & 3,5-difluoro & $\mathrm{CH}_{3}$ & $\begin{array}{l}\text { Angew. Chem. Int. Ed. 2017, 56, } \\
5111\end{array}$ \\
\hline 1n & $p$-CN & $\mathrm{CH}_{3}$ & Chem. Eur. J. 2017, 23, 15534 \\
\hline 10 & thiophen-3-yl & $\mathrm{CH}_{3}$ & $\begin{array}{l}\text { Angew. Chem. Int. Ed. 2017, 56, } \\
5111\end{array}$ \\
\hline $3 \mathbf{a}$ & cyclohex-1-en-1-yl & $\mathrm{CH}_{3}$ & Org. Lett. 2019, 21, 6795 \\
\hline 3b & cyclohexan-1-yl & $\mathrm{CH}_{3}$ & Org. Lett. 2019, 21, 6795 \\
\hline 3d & cycloprop-1-yl & $\mathrm{CH}_{3}$ & Org. Lett. 2019, 21, 6795 \\
\hline $\mathbf{3 e}$ & $\mathrm{H}$ & $\mathrm{CH}_{3}$ & J. Org. Chem. 1985, 50, 3979 \\
\hline $5 \mathbf{a}$ & $\mathrm{H}$ & $\mathrm{H}$ & Commercially Available \\
\hline $5 \mathbf{b}$ & $p-\mathrm{C}\left(\mathrm{CH}_{3}\right)_{3}$ & $\mathrm{H}$ & Org. Lett. 2019, 21, 6795 \\
\hline $\mathbf{5 c}$ & $p-\mathrm{CF}_{3}$ & $\mathrm{H}$ & Org. Lett. 2019, 21, 6795 \\
\hline $5 f$ & $\begin{array}{l}\text { 5-methylthiophen- } \\
\text { 2-yl }\end{array}$ & $\mathrm{H}$ & Org. Lett. 2019, 21, 6795 \\
\hline
\end{tabular}




\section{Characterization of Propiolamides:}

tert-butyl (3-(2-chlorophenyl)propioloyl)glycinate (1d)<smiles>CC(C)(C)OC(=O)CNC(=O)C#Cc1ccccc1Cl</smiles>

Prepared according to Procedure 3.3. White solid (36 mg, $0.123 \mathrm{mmol}$, $14 \%) . \mathbf{R}_{\mathbf{f}}(3: 2$ hexanes: ethyl acetate $)=0.41 .{ }^{1} \mathbf{H} \mathbf{~ N M R}\left(\mathrm{CDCl}_{3}, 400 \mathrm{MHz}\right)$ $\delta 7.54-7.49(\mathrm{dd}, \mathrm{J}=7.6,1.7 \mathrm{~Hz}, 1 \mathrm{H}), 7.39-7.34(\mathrm{~m}, 1 \mathrm{H}), 7.33-7.27$ $(\mathrm{dt}, \mathrm{J}=7.4,1.8 \mathrm{~Hz}, 1 \mathrm{H}), 7.23-7.18(\mathrm{dt}, \mathrm{J}=7.5,1.5 \mathrm{~Hz}, 1 \mathrm{H}), 6.66-6.61$ $(\mathrm{t}, \mathrm{J}=5.3 \mathrm{~Hz}, 1 \mathrm{H}), * 6.42-6.36(\mathrm{t}, \mathrm{J}=6.0 \mathrm{~Hz}, 1 \mathrm{H}), * 4.21-4.18(\mathrm{~d}, \mathrm{~J}=$ $6.2 \mathrm{~Hz}, 2 \mathrm{H}), 4.04-3.98(\mathrm{~d}, \mathrm{~J}=5.0 \mathrm{~Hz}, 2 \mathrm{H}), 1.48-1.44(\mathrm{~s}, 9 \mathrm{H}), * 1.44-$ 1.41 (s, 9H). ${ }^{13} \mathbf{C} \mathbf{N M R}\left(\mathrm{CDCl}_{3}, 100 \mathrm{MHz}\right) \delta 168.3, * 168.1, * 155.7,152.9, * 137.0,136.9, * 134.7,134.4$, $* 131.6,131.2,129.5, * 126.9,126.7,120.3, * 87.2,87.0,82.8,81.8, * 45.5,42.3,28.1$. HRMS (ESI-TOF) $\mathrm{m} / \mathrm{z}:[\mathrm{M}+\mathrm{H}]^{+} \mathrm{Calcd}$ for $\mathrm{C}_{15} \mathrm{H}_{17} \mathrm{ClNO}_{3}{ }^{+}$294.0891; Found 294.0894.

\section{$N$-methyl-3-(4-(trifluoromethoxy)phenyl)propiolamide (11)}

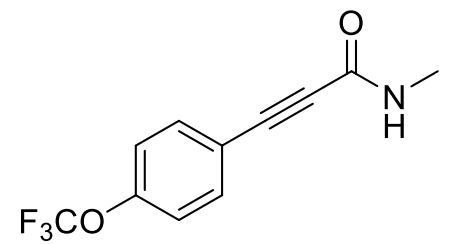

Prepared according to Procedure 3.2. Off-white solid $(64.2 \mathrm{mg}, 0.264$ mmol, 32\%). $\mathbf{R}_{\mathbf{f}}(3: 2$ dichloromethane:ethyl acetate $)=0.76 .{ }^{1} \mathbf{H}$ NMR (Acetone- $\left.d_{6}, 500 \mathrm{MHz}\right) \delta 7.79(\mathrm{~s}, 1 \mathrm{H}), * 7.79-7.76(\mathrm{~m}, 2 \mathrm{H}), 7.72-7.64(\mathrm{~m}$, $2 \mathrm{H}), * 7.45-7.42(\mathrm{~d}, \mathrm{~J}=9.0 \mathrm{~Hz}, 2 \mathrm{H}), 7.43-7.37(\mathrm{~d}, \mathrm{~J}=8.0 \mathrm{~Hz}, 2 \mathrm{H}), * 3.08$ $-3.07(\mathrm{~d}, \mathrm{~J}=4.8 \mathrm{~Hz}, 3 \mathrm{H}), 2.82-2.80(\mathrm{~d}, \mathrm{~J}=4.9 \mathrm{~Hz}, 3 \mathrm{H}) ;{ }^{19} \mathbf{F}$ NMR (Acetone- $\left.d_{6}, 376 \mathrm{MHz}\right) \delta-58.59 ;{ }^{13} \mathbf{C}$ NMR (Acetone- $\left.d_{6}, 126 \mathrm{MHz}\right) \delta 153.7$, $150.7(\mathrm{q}, \mathrm{J}=1.9 \mathrm{~Hz}), * 135.3,135.2,122.2,121.3(\mathrm{q}, \mathrm{J}=257 \mathrm{~Hz}), 120.7,85.2, * 82.9,81.8,26.3$. HRMS (ESI-TOF) m/z: [M+H] $]^{+}$Calcd for $\mathrm{C}_{11} \mathrm{H}_{9} \mathrm{~F}_{3} \mathrm{NO}_{2}{ }^{+}$244.0579; Found 244.0571.

\section{$N$-methyl-3-(pyridin-3-yl)propiolamide (1p)}

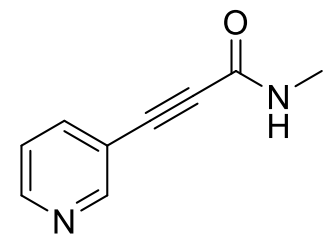

Substrate 5h was synthesized using an adapted protocol. ${ }^{1} \mathrm{~N}$-methylpropiolamide (223 mg, $2.69 \mathrm{mmol}$ ) was added to a 6 dram vial, purged with $\mathrm{N}_{2}$ via Schlenk technique, and then diluted with THF $(3.4 \mathrm{~mL})$. To a $10 \mathrm{~mL}$ round bottom flask was added $\mathrm{Pd}\left(\mathrm{PPh}_{3}\right)_{4}(141 \mathrm{mg}, 0.122 \mathrm{mmol})$ and 3-iodopyridine $(500 \mathrm{mg}, 2.44 \mathrm{mmol})$ which was subsequently purged with $\mathrm{N}_{2}$ via Schlenk technique. To this mixture was added THF (1.6 mL) and DBU $(4.88 \mathrm{mmol}, 0.729 \mathrm{~mL})$. The mixture was purged with $\mathrm{N}_{2}$ for an additional 30 minutes. The $N$-methylpropiolamide solution was then added to the $10 \mathrm{~mL}$ round bottom flask and the resulting mixture was stirred at $35^{\circ} \mathrm{C}$ for 18 hours. The reaction mixture was diluted with ethyl acetate $(10 \mathrm{~mL})$. The organic layer was washed with water and brine then dried over anhydrous sodium sulfate. The organic layer was filtered and concentrated in vacuo. The resulting crude product was purified by silica gel column chromatography (eluted with a $30-60 \%$ gradient ethyl acetate in dichloromethane). Light orange solid (221 mg, $1.38 \mathrm{mmol}, 56 \%)$. $\mathbf{R}_{\mathbf{f}}(3: 2$ dichloromethane:ethyl acetate) $=0.15 .{ }^{1} \mathrm{H}$ NMR $\left(\mathrm{CD}_{3} \mathrm{OD}, 400 \mathrm{MHz}\right) \delta * 8.79-8.78(\mathrm{dd}, \mathrm{J}=2.1,1.0 \mathrm{~Hz}, 1 \mathrm{H}), 8.76-8.69(\mathrm{dd}, \mathrm{J}=2.4$, $0.9 \mathrm{~Hz}, 1 \mathrm{H}), * 8.64-8.62(\mathrm{dd}, \mathrm{J}=8.6,1.7 \mathrm{~Hz}, 1 \mathrm{H}), 8.62-8.57(\mathrm{dd}, \mathrm{J}=5.0,1.7 \mathrm{~Hz}, 1 \mathrm{H})$, *8.08 - 8.05 (ddd, $\mathrm{J}=8.0,2.2,1.9 \mathrm{~Hz}, 1 \mathrm{H}), 8.03-7.98(\mathrm{ddd}, \mathrm{J}=8.0,2.1,2.0 \mathrm{~Hz}, 1 \mathrm{H}), * 7.53-7.49(\mathrm{ddd}, \mathrm{J}=7.8,5.0,1.0 \mathrm{~Hz}$, 1H), $7.51-7.46(\mathrm{ddd}, \mathrm{J}=8.1,5.0,1.0 \mathrm{~Hz}, 1 \mathrm{H}), * 3.08(\mathrm{~s}, 3 \mathrm{H}), 2.8(\mathrm{~s}, 3 \mathrm{H}) .{ }^{13} \mathbf{C}$ NMR (Acetone- $d_{6}, 100$ MHz) $\delta * 153.5,153.3, * 151.3,151.1, * 140.2,140.0,124.4,118.6,87.2, * 87.2$, *80.3, 80.3, 26.4, 26.2. HRMS (ESI-TOF) m/z: [M+H] $]^{+}$Calcd for $\mathrm{C}_{9} \mathrm{H}_{9} \mathrm{~N}_{2} \mathrm{O}^{+}$161.0709; Found 161.0709. 


\section{7-(benzyloxy)- $N$-methylhept-2-ynamide (3c)}

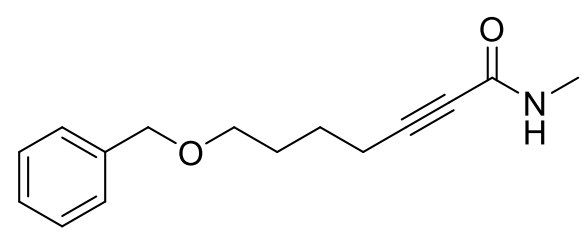

Prepared according to Procedure 3.1. Clear oil, (291 mg, 1.19 mmol, 71\%). $\mathbf{R}_{\mathbf{f}}(3: 2$ hexanes:ethyl acetate $)=0.08 .{ }^{1} \mathbf{H}$ NMR $\left(\mathrm{CDCl}_{3}, 400 \mathrm{MHz}\right) \delta 7.36-7.24(\mathrm{~m}, 5 \mathrm{H}), 5.95-5.81(\mathrm{~s}, 1 \mathrm{H}), * 5.78$ $-5.69(\mathrm{~s}, 1 \mathrm{H}), 4.48(\mathrm{~s}, 2 \mathrm{H}), 3.50-3.46(\mathrm{t}, \mathrm{J}=6.0 \mathrm{~Hz}, 2 \mathrm{H}), * 2.98-$ $2.96(\mathrm{~d}, \mathrm{~J}=5.2 \mathrm{~Hz}, 3 \mathrm{H}), 2.82-2.79(\mathrm{~d}, \mathrm{~J}=5.1 \mathrm{~Hz}, 3 \mathrm{H}), * 2.42-$ $2.38(\mathrm{t}, \mathrm{J}=7.0 \mathrm{~Hz}, 2 \mathrm{H}), 2.33-2.28(\mathrm{t}, \mathrm{J}=7.0 \mathrm{~Hz}, 2 \mathrm{H}), 1.75-1.60$ $(\mathrm{m}, 4 \mathrm{H}) .{ }^{13} \mathbf{C}$ NMR $\left(\mathrm{CDCl}_{3}, 100 \mathrm{MHz}\right) \delta 154.2,138.4,128.4,127.6,127.6,86.9,75.8,73.0,69.7, * 69.6$, $* 29.0,28.9,26.5$, *24.8, 24.7, 18.8, 18.5. HRMS (ESI-TOF) $\mathrm{m} / \mathrm{z}$ : $[\mathrm{M}+\mathrm{H}]^{+}$Calcd for $\mathrm{C}_{15} \mathrm{H}_{20} \mathrm{NO}_{2}{ }^{+} 246.1489$; Found 246.1477.

\section{3-(dimethyl(phenyl)silyl)- $N$-methylpropiolamide (3f)}<smiles>CNC(=O)C#C[Si](C)(C)c1ccccc1</smiles>

Substrate 3f was synthesized using an adapted protocol. ${ }^{2}$ To a flame-dried $15 \mathrm{~mL}$ round bottom flask was added $N$-methylpropiolamide $(415 \mathrm{mg}, 5.00 \mathrm{mmol})$ that was subsequently purged with $\mathrm{N}_{2}$ via Schlenk technique. THF $(9.0 \mathrm{~mL})$ was added and the mixture cooled to $-78^{\circ} \mathrm{C}$ with a dry ice/acetone bath. After cooling for 15 minutes, LiHMDS (5.00 mmol, 1.0 M in THF) was added dropwise. The mixture was stirred for $30 \mathrm{~min}$ at $-78{ }^{\circ} \mathrm{C}$, after which chlorodimethyl(phenyl)silane $(5.00 \mathrm{mmol}, 0.839 \mathrm{~mL})$ was added and the mixture allowed to warm to room temperature overnight. The mixture was poured into sat. $\mathrm{NH}_{4} \mathrm{Cl}(5 \mathrm{~mL})$ and was extracted with diethyl ether $(2 \times 5 \mathrm{~mL})$. The organic layer was washed with brine $(5 \mathrm{~mL})$, dried over anhydrous sodium sulfate, filtered, and concentrated in vacuo. The residue was purified by silica gel column chromatography (eluted with a 10-30\% gradient ethyl acetate in dichloromethane). Off-white solid, $(627 \mathrm{mg}, 5.00 \mathrm{mmol}, 58 \%)$. $\mathbf{R}_{\mathbf{f}}(3: 2$ dichloromethane:ethyl acetate $)=$ 0.32. ${ }^{1} \mathbf{H}$ NMR $\left(\mathrm{CDCl}_{3}, 400 \mathrm{MHz}\right) \delta 7.63-7.58(\mathrm{~m}, 2 \mathrm{H}), 7.44-7.35(\mathrm{~m}, 3 \mathrm{H}), 5.95-5.73(\mathrm{~s}, 1 \mathrm{H}), * 5.61-$ $5.53(\mathrm{~s}, 1 \mathrm{H}), * 3.05-3.03(\mathrm{~d}, \mathrm{~J}=5.4 \mathrm{~Hz}, 3 \mathrm{H}), 2.87-2.84(\mathrm{~d}, \mathrm{~J}=5.0 \mathrm{~Hz}, 3 \mathrm{H}), 0.48-0.46(\mathrm{~s}, 6 \mathrm{H}) .{ }^{13} \mathbf{C ~ N M R}$ $\left(\mathrm{CDCl}_{3}, 100 \mathrm{MHz}\right) \delta 153.2,135.0,133.7,129.9,128.1,99.0,89.4,26.6,-1.4$. HRMS (ESI-TOF) m/z: $[\mathrm{M}+\mathrm{H}]^{+}$Calcd for $\mathrm{C}_{12} \mathrm{H}_{16} \mathrm{NOSi}^{+}$218.0996; Found 218.0987.

\section{3-(4-(trifluoromethoxy)phenyl)propiolamide (5d)}

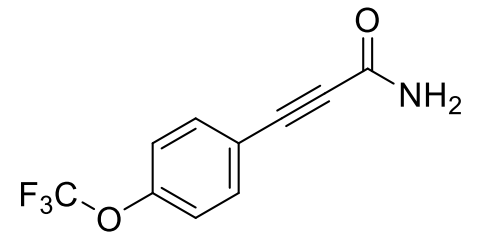

Prepared according to Procedure 3.2. Off-white solid, (58.3 mg, 0.254 mmol, 66\%). $\mathbf{R}_{\mathbf{f}}(3: 2$ dichloromethane:ethyl acetate $)=0.68 .{ }^{1} \mathbf{H}$ NMR (Acetone- $\left.d_{6}, 500 \mathrm{MHz}\right) \delta 7.72-7.68(\mathrm{~m}, 2 \mathrm{H}), 7.54(\mathrm{~s}, 1 \mathrm{H}), 7.41(\mathrm{~d}, \mathrm{~J}=$ $7.9 \mathrm{~Hz}, 2 \mathrm{H}), 7.03(\mathrm{~s}, 1 \mathrm{H}) ;{ }^{19} \mathbf{F}$ NMR (Acetone- $\left.d_{6}, 376 \mathrm{MHz}\right) \delta-58.20--$ $58.31(\mathrm{~m}) ;{ }^{13} \mathbf{C}$ NMR (Acetone- $\left.d_{6}, 126 \mathrm{MHz}\right) \delta 154.7,150.7$ (q, J = 2.2 $\mathrm{Hz}$ ), 135.2, 122.2, 121.3 (q, J = $257 \mathrm{~Hz}), 120.6$, 85.1, 82.3; HRMS (ESI-

TOF) m/z: $[\mathrm{M}+\mathrm{H}]^{+}$Calcd for $\mathrm{C}_{10} \mathrm{H}_{7} \mathrm{~F}_{3} \mathrm{NO}_{2}{ }^{+}$230.0423; Found 230.0417. 


\section{$N$-methyl-3-(4-nitrophenyl)propiolamide (5e)}<smiles>NC(=O)C#Cc1ccc([N+](=O)[O-])cc1</smiles>

Prepared according to Procedure 3.2. Light yellow solid, (20.4 mg, 0.100 mmol, 24\%). $\mathbf{R}_{\mathbf{f}}$ (3:2 dichloromethane:ethyl acetate) $=0.59 .{ }^{1} \mathbf{H}$ NMR (Acetone- $\left.d_{6}, 400 \mathrm{MHz}\right) \delta 8.34-8.29(\mathrm{~m}, 2 \mathrm{H}), 7.87-7.82(\mathrm{~m}, 2 \mathrm{H}), 7.60(\mathrm{~s}$, $1 \mathrm{H}), 7.08(\mathrm{~s}, 1 \mathrm{H}) ;{ }^{13} \mathrm{C}$ NMR (Acetone- $\left.d_{6}, 126 \mathrm{MHz}\right) \delta 154.0,149.2,134.21$, 128.0, 124.7, 87.9, 81.5; HRMS (ESI-TOF) $\mathrm{m} / \mathrm{z}:[\mathrm{M}+\mathrm{H}]^{+}$Calcd for $\mathrm{C}_{9} \mathrm{H}_{7} \mathrm{~N}_{2} \mathrm{O}_{3}{ }^{+}$191.0451; Found 191.0450.

\section{3-(pyridin-2-yl)propiolamide (5g)}

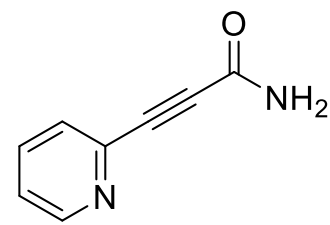

Prepared according to Procedure 3.1. Purified using a $1-4 \%$ gradient methanol in dichloromethane. Off-white solid, (49.0 mg, $0.335 \mathrm{mmol}, 36 \%)$. $\mathbf{R}_{\mathbf{f}}(95: 5$ dichloromethane:methanol) $=0.59 .{ }^{1} \mathbf{H}$ NMR $\left(\mathrm{CD}_{3} \mathrm{OD}, 400 \mathrm{MHz}\right) \delta 8.62-8.57$ $(\mathrm{ddd}, \mathrm{J}=4.9,1.8,0.9 \mathrm{~Hz}, 1 \mathrm{H}), 7.93-7.87(\mathrm{dt}, \mathrm{J}=7.8,1.8 \mathrm{~Hz}, 1 \mathrm{H}), 7.72-7.68(\mathrm{dt}$, $\mathbf{J}=7.8,1.1 \mathrm{~Hz}, 1 \mathrm{H}), 7.52-7.47(\mathrm{ddd}, \mathrm{J}=7.7,4.9,1.1 \mathrm{~Hz}, 1 \mathrm{H}) .{ }^{13} \mathbf{C} \mathbf{N M R}\left(\mathrm{CD}_{3} \mathrm{OD}\right.$, $100 \mathrm{MHz}) \delta 156.6,151.2,141.7,138.7,129.8,126.2,83.6,83.0$. HRMS (ESI-TOF) $\mathrm{m} / \mathrm{z}:[\mathrm{M}+\mathrm{H}]^{+}$Calcd for $\mathrm{C}_{8} \mathrm{H}_{7} \mathrm{~N}_{2} \mathrm{O}^{+}$147.0553; Found 147.0552.

\section{3-(pyridin-3-yl)propiolamide (5h)}<smiles>NC(=O)C#Cc1cccnc1</smiles>

Prepared according to Procedure 3.2. Purified using a $3-5 \%$ gradient methanol in dichloromethane. Off-white solid (254 mg, $1.74 \mathrm{mmol}, \quad 78 \%)$. $\mathbf{R}_{\mathbf{f}} \quad(3: 2$ dichloromethane:ethyl acetate $)=0.21 .{ }^{1} \mathbf{H} \mathbf{~ N M R}\left(\mathrm{CD}_{3} \mathrm{OD}, 400 \mathrm{MHz}\right) \delta 8.76-8.72$ $(\mathrm{dd}, \mathrm{J}=2.2,0.9 \mathrm{~Hz}, 1 \mathrm{H}), 8.63-8.59(\mathrm{dd}, \mathrm{J}=5.0,1.7 \mathrm{~Hz}, 1 \mathrm{H}), 8.04-8.00$ (ddd, J $=8.0,2.1,1.6 \mathrm{~Hz}, 1 \mathrm{H}), 7.52-7.47$ (ddd, J = 7.9, 5.0, 0.9 Hz, 1H). ${ }^{13} \mathbf{C}$ NMR $\left(\mathrm{CD}_{3} \mathrm{OD}, 100 \mathrm{MHz}\right) \delta 156.9,153.4,151.0,141.5,125.1,119.5,86.8,82.1$. HRMS (ESI-TOF) m/z: [M+H] ${ }^{+}$Calcd for $\mathrm{C}_{8} \mathrm{H}_{7} \mathrm{~N}_{2} \mathrm{O}, 147.0553$; Found 147.0554.

\section{3-(pyridin-3-yl)propiolamide (5i)}

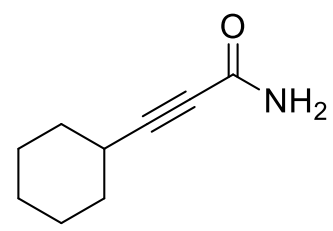

Prepared according to Procedure 3.1. White solid (239 mg, $1.58 \mathrm{mmol}, 58 \%$ ). Purified using a $0-10 \%$ gradient ethyl acetate in dichloromethane. $\mathbf{R}_{\mathbf{f}}$ (9:1 dichloromethane:ethyl acetate $)=0.31 .{ }^{1} \mathbf{H} \mathbf{~ N M R}\left(\mathrm{CDCl}_{3}, 400 \mathrm{MHz}\right) \delta 5.71-5.52$ $(\mathrm{s}, 2 \mathrm{H}), 2.52-2.44(\mathrm{~m}, 1 \mathrm{H}), 1.87-1.79(\mathrm{~m}, 2 \mathrm{H}), 1.76-1.66(\mathrm{~m}, 2 \mathrm{H}), 1.57-1.43$ (m, 4H) $1.38-1.26(\mathrm{~m}, 4 \mathrm{H}) .{ }^{13} \mathbf{C}$ NMR $\left(\mathrm{CDCl}_{3}, 100 \mathrm{MHz}\right) \delta 155.6,92.6,74.9$, 31.7, 28.9, 25.7, 24.8. HRMS (ESI-TOF) $\mathrm{m} / \mathrm{z}$ : $[\mathrm{M}+\mathrm{H}]^{+}$Calcd for $\mathrm{C}_{9} \mathrm{H}_{14} \mathrm{NO}^{+}$ 152.1070; Found 152.1069. 


\section{General Procedures for Semi-reduction of Propiolamides:}

Procedure 5.1 An oven- or flame-dried $5 \mathrm{~mL}$ round bottom flask was charged with dry propiolamide (32 $\mathrm{mg}, 0.2 \mathrm{mmol})$ and placed under $\mathrm{N}_{2}$ via Schlenk technique. The reaction was diluted with THF $(0.4 \mathrm{~mL}$, $0.5 \mathrm{M}$ alkynamide $)$ and stirred at room temperature. $\mathrm{KO} t \mathrm{Bu}(0.02 \mathrm{mmol})$ was added as a solid to the round bottom flask. Pinacolborane $(0.032 \mathrm{~mL}, 0.22 \mathrm{mmol})$ was then slowly added dropwise (1 drop every $3-4$ seconds). This order of addition is important to obtain high yield, regio- and stereoselectivity. The reaction was stirred at room temperature for 10 minutes. The reaction mixture was concentrated in vacuo and the resulting crude residue was purified by silica gel column chromatography (eluted with a $30-40 \%$ gradient ethyl acetate in dichloromethane) to afford the product as a white solid. Where applicable, the $E$-isomer is labeled as major when the $Z$-isomer is present, which is labeled as minor.

Procedure 5.2 An oven- or flame-dried $5 \mathrm{~mL}$ round bottom flask was charged with dry propiolamide (30 $\mathrm{mg}, 0.21 \mathrm{mmol}$ ) and placed under $\mathrm{N}_{2}$ via Schlenk technique. The reaction was diluted with THF (1.0 mL, $0.2 \mathrm{M}$ propiolamide) and stirred at room temperature. $\mathrm{KO} t \mathrm{Bu}(0.21 \mathrm{mmol})$ was added as a solid to the round bottom flask. Pinacolborane $(0.033 \mathrm{~mL}, 0.23 \mathrm{mmol})$ was then slowly added dropwise (1 drop every 3-4 seconds). This order of addition is important to obtain high yield, regio- and stereoselectivity. The reaction was stirred at room temperature for 10 minutes. The reaction mixture was concentrated in vacuo and the resulting crude residue was purified by silica gel column chromatography (eluted with a 30-40\% gradient ethyl acetate in dichloromethane) to afford the product as a white solid. Where applicable, the $E$-isomer is labeled as major when the Z-isomer is present, which is labeled as minor.

\section{Characterization of $E$-cinnamamides:}

\section{(E)- $N$-methyl-3-phenylacrylamide (2a)}<smiles>CNC(=O)/C=C/c1ccccc1</smiles>

Prepared according to Procedure 5.1. White solid (33.7 mg, $0.209 \mathrm{mmol}, 96 \%$, $>99: 1 \mathrm{E:Z}) . \mathbf{R}_{\mathbf{f}}(3: 2$ dichloromethane:ethyl acetate $)=0.38 .{ }^{\mathbf{1}} \mathbf{H} \mathbf{~ N M R}\left(\mathrm{CDCl}_{3}, 400\right.$ MHz) $\delta 7.65-7.60(\mathrm{~d}, \mathrm{~J}=15.9 \mathrm{~Hz}, 1 \mathrm{H}), 7.51-7.45(\mathrm{~m}, 2 \mathrm{H}), 7.37-7.30(\mathrm{~m}, 3 \mathrm{H})$, $6.46-6.40(\mathrm{~d}, \mathrm{~J}=15.7 \mathrm{~Hz}, 1 \mathrm{H}), 6.05-5.96(\mathrm{~s}, 1 \mathrm{H}), 2.96-2.92(\mathrm{~d}, \mathrm{~J}=5.0 \mathrm{~Hz}, 3 \mathrm{H})$. ${ }^{13} \mathbf{C ~ N M R}\left(\mathrm{CDCl}_{3}, 100 \mathrm{MHz}\right) \delta 166.8,140.8,135.0,129.7,128.9,127.9,120.8,26.6$.

HRMS (ESI-TOF) m/z: [M+H] $]^{+}$Calcd for $\mathrm{C}_{10} \mathrm{H}_{12} \mathrm{NO}^{+}$162.0913; Found 162.0897.

$3.2 \mathrm{mmol}$ scale: White solid (497 mg, $3.2 \mathrm{mmol}, 97 \%,>99: 1 \mathrm{E:Z})$

\section{Procedure for $3.2 \mathrm{mmol}$ scale:}

A flame-dried $15 \mathrm{~mL}$ round bottom flask was charged with dry propiolamide (509 $\mathrm{mg}, 3.2 \mathrm{mmol}$ ) and placed under $\mathrm{N}_{2}$ via Schlenk technique. The reaction was diluted with THF $(5.0 \mathrm{~mL})$ and stirred at room temperature. $\mathrm{KO} t \mathrm{Bu}(36 \mathrm{mg}, 0.32 \mathrm{mmol})$ was added as a solid. Pinacolborane $(0.51 \mathrm{~mL}, 3.5 \mathrm{mmol})$ was then slowly added dropwise (1 drop every 3-4 seconds). The reaction was stirred at room temperature for 10 minutes. The reaction mixture was concentrated in vacuo and the resulting crude residue was purified by silica gel column chromatography (eluted with a 30-40\% gradient ethyl acetate in dichloromethane) to afford the product as a white solid.

\section{(E)- $N$-isopropylcinnamamide (2b)}<smiles>CC(C)NC(=O)/C=C/c1ccccc1</smiles>

Prepared according to Procedure 5.1. White solid (24.5 mg, $0.129 \mathrm{mmol}, 69 \%$, $>99: 1 E: Z)$. $\mathbf{R}_{\mathbf{f}}(9: 1$ dichloromethane:ethyl acetate $)=0.40$. Purified using a 0-10\% gradient ethyl acetate in dichloromethane. ${ }^{1} \mathbf{H} \mathbf{~ N M R}\left(\mathrm{CDCl}_{3}, 400 \mathrm{MHz}\right) \delta 7.64-$ 7.58 (major, d, J = $15.7 \mathrm{~Hz}, 1 \mathrm{H}), 7.50-7.44(\mathrm{~m}, 2 \mathrm{H}), 7.36-7.29(\mathrm{~m}, 3 \mathrm{H}), 6.76$ 
- 6.72 (minor, d, J = 12.6 Hz, 1H), $6.45-6.36$ (major, d, J = 15.2 Hz, 1H), $6.00-5.96$ (minor, d, J = 13.0 $\mathrm{Hz}, 1 \mathrm{H}), 5.83-5.68(\mathrm{~d}, \mathrm{~J}=6.8 \mathrm{~Hz}, 1 \mathrm{H}), 4.29-4.16(\mathrm{~m}, 1 \mathrm{H}), 1.24-1.19(\mathrm{~d}, \mathrm{~J}=7.0 \mathrm{~Hz}, 6 \mathrm{H}) .{ }^{13} \mathbf{C} \mathbf{~ N M R}$ $\left(\mathrm{CDCl}_{3}, 100 \mathrm{MHz}\right) \delta 165.2,140.8,135.1,129.6,128.9,127.8,121.3,41.7,22.9$. HRMS (ESI-TOF) m/z: $[\mathrm{M}+\mathrm{H}]^{+}$Calcd for $\mathrm{C}_{12} \mathrm{H}_{16} \mathrm{NO}^{+}$190.1226; Found 190.1220.

\section{$(E)-N$-phenylcinnamamide (2c)}<smiles>O=C(/C=C/c1ccccc1)Nc1ccccc1</smiles>

Prepared according to Procedure 5.1. Off-white solid (48.2 mg, $0.216 \mathrm{mmol}$, $81 \%,>99: 1 E: Z)$. $\mathbf{R}_{\mathbf{f}}(3: 2$ dichloromethane:ethyl acetate $)=0.91$. Purified using a $100 \%$ isocratic dichloromethane solvent system. ${ }^{1} \mathbf{H}$ NMR $\left(\mathrm{CDCl}_{3}, 400\right.$ $\mathrm{MHz}) \delta 7.78-7.71$ (major, d, J = $15.6 \mathrm{~Hz}, 1 \mathrm{H}), 7.75-7.71(\mathrm{~s}, 1 \mathrm{H}), 7.67-$ $7.61(\mathrm{~m}, 2 \mathrm{H}), 7.51-7.46(\mathrm{~m}, 2 \mathrm{H}), 7.38-7.31(\mathrm{~m}, 5 \mathrm{H}), 7.17-7.08(\mathrm{t}, \mathrm{J}=7.4$ $\mathrm{Hz}, 1 \mathrm{H}$ ), $6.94-6.90$ (minor, d, J = $12.5 \mathrm{~Hz}, 1 \mathrm{H}$ ), $6.65-6.54$ (major, d, J = $15.3 \mathrm{~Hz}, 1 \mathrm{H}$ ), $6.14-6.10$ (minor, $\mathrm{d}, \mathrm{J}=12.2 \mathrm{~Hz}, 1 \mathrm{H}) .{ }^{13} \mathbf{C} \mathbf{N M R}\left(\mathrm{CDCl}_{3}, 100 \mathrm{MHz}\right) \delta 164.3,142.5,138.2,134.7,130.1,129.2,129.0$, 128.1, 124.6, 121.0, 120.2. HRMS (ESI-TOF) $\mathrm{m} / \mathrm{z}:[\mathrm{M}+\mathrm{H}]^{+}$Calcd for $\mathrm{C}_{15} \mathrm{H}_{14} \mathrm{NO}^{+}$224.1070; Found 224.1068 .

tert-butyl (E)-(3-(2-chlorophenyl)acryloyl)glycinate (2d)<smiles>CC(C)(C)OC(=O)CNC(=O)/C=C/c1ccccc1Cl</smiles>

Prepared according to Procedure 5.1. Off-white solid (37.0 mg, 0.130 mmol, 73\%, >99:1 E:Z). $\mathbf{R}_{\mathbf{f}}(3: 2$ dichloromethane:ethyl acetate $)=0.59$. Purified using a 10-20\% gradient ethyl acetate in dichloromethane. ${ }^{1} \mathbf{H}$ NMR $\left(\mathrm{CDCl}_{3}, 400 \mathrm{MHz}\right) \delta 8.03-7.95(\mathrm{~d}, \mathrm{~J}=16.0 \mathrm{~Hz}, 1 \mathrm{H}), 7.58-7.53$ $(\mathrm{m}, 1 \mathrm{H}), 7.41-7.34(\mathrm{~m}, 1 \mathrm{H}), 7.30-7.21(\mathrm{~m}, 2 \mathrm{H}), 6.52-6.40(\mathrm{~d}, \mathrm{~J}=$ $15.7 \mathrm{~Hz}, 1 \mathrm{H}), 6.32-6.26(\mathrm{~s}, 1 \mathrm{H}), 4.10-4.06(\mathrm{~d}, \mathrm{~J}=4.9 \mathrm{~Hz}, 2 \mathrm{H}), 1.51-1.46(\mathrm{~s}, 9 \mathrm{H}){ }^{13}{ }^{13} \mathrm{NMR}\left(\mathrm{CDCl}_{3}\right.$, $100 \mathrm{MHz}) \delta 169.3,165.4,137.6,134.9,133.2,130.7,130.3,127.7,127.1,123.1,82.7,42.4,28.2$. HRMS (ESI-TOF) m/z: [M+H] ${ }^{+}$Calcd for $\mathrm{C}_{15} \mathrm{H}_{19} \mathrm{ClNO}_{3}{ }^{+}$296.1048; Found 296.1057.

\section{(E)-N-(prop-2-yn-1-yl)cinnamamide (2e)}<smiles>C#CCNC(=O)/C=C/c1ccccc1</smiles>

Prepared according to Procedure 5.1. Off-white solid ( $40.0 \mathrm{mg}, 0.220 \mathrm{mmol}$, $79 \%,>99: 1 E: Z)$. $\mathbf{R}_{\mathbf{f}}(3: 2$ dichloromethane:ethyl acetate $)=0.83$. Purified using a $40-60 \%$ gradient ethyl acetate in hexanes. ${ }^{1} \mathbf{H}$ NMR $\left(\mathrm{CDCl}_{3}, 400 \mathrm{MHz}\right) \delta$ $7.79-7.63$ (major, d, J = $15.9 \mathrm{~Hz}, 1 \mathrm{H}), 7.52-7.45(\mathrm{~m}, 2 \mathrm{H}), 7.39-7.32(\mathrm{~m}$, $3 \mathrm{H}$ ), $6.83-6.79$ (minor, d, J = $13.5 \mathrm{~Hz}, 1 \mathrm{H}$ ), $6.46-6.40$ (major, $\mathrm{d}, \mathrm{J}=15.8$ $\mathrm{Hz}, 1 \mathrm{H}), 6.14-6.01(\mathrm{~s}, 1 \mathrm{H}), 4.22-4.18(\mathrm{dd}, \mathrm{J}=5.0,2.6 \mathrm{~Hz}, 2 \mathrm{H}), 2.27-2.25(\mathrm{t}, \mathrm{J}=2.8 \mathrm{~Hz}, 1 \mathrm{H}) .{ }^{13} \mathbf{C ~ N M R}$ $\left(\mathrm{CDCl}_{3}, 100 \mathrm{MHz}\right) \delta 165.7,142.0,134.7,130.0,129.0,128.0,119.9,79.6,71.9,29.6$. HRMS (ESI-TOF) $\mathrm{m} / \mathrm{z}:[\mathrm{M}+\mathrm{H}]^{+}$Calcd for $\mathrm{C}_{24} \mathrm{H}_{23} \mathrm{~N}_{2} \mathrm{O}_{2}{ }^{+}$371.1754; Found 371.1730.

\section{(E)-3-(4-ethoxyphenyl)- $N$-methylacrylamide (2f)}<smiles>CCOc1ccc(/C=C/C(=O)NC)cc1</smiles>

Prepared according to Procedure 5.1. White solid (35.5 mg, $0.173 \mathrm{mmol}$, $89 \%,>99: 1 E: Z)$. $\mathbf{R}_{\mathbf{f}}(3: 2$ dichloromethane:ethyl acetate $)=0.32 .{ }^{1} \mathbf{H} \mathbf{~ N M R}$ $\left(\mathrm{CDCl}_{3}, 400 \mathrm{MHz}\right) \delta 7.57-7.52(\mathrm{~d}, \mathrm{~J}=15.3 \mathrm{~Hz}, 1 \mathrm{H}), 7.41-7.36(\mathrm{~d}, \mathrm{~J}=$ $8.3 \mathrm{~Hz}, 2 \mathrm{H}), 6.84-6.78(\mathrm{~d}, \mathrm{~J}=8.8 \mathrm{~Hz}), 6.32-6.26(\mathrm{~d}, \mathrm{~J}=15.6 \mathrm{~Hz}, 1 \mathrm{H})$, $4.05-3.97(\mathrm{q}, \mathrm{J}=6.9 \mathrm{~Hz}, 2 \mathrm{H}), 2.92-2.87(\mathrm{~s}, 3 \mathrm{H}), 1.42-1.36(\mathrm{t}, \mathrm{J}=6.6$ $\mathrm{Hz}, 3 \mathrm{H}) .{ }^{13} \mathrm{C}$ NMR $\left(\mathrm{CDCl}_{3}, 100 \mathrm{MHz}\right) \delta 167.1,160.2,140.3,129.3,127.4,118.1,114.7,63.5,26.3,14.7$. HRMS (ESI-TOF) m/z: [M+H] ${ }^{+}$Calcd for $\mathrm{C}_{12} \mathrm{H}_{16} \mathrm{NO}_{2}{ }^{+}$206.1176; Found 206.1155. 


\section{(E)-3-(2-methoxyphenyl)- $N$-methylacrylamide (2g)}<smiles>CNC(=O)/C=C/c1ccccc1OC</smiles>

Prepared according to Procedure 5.1. White solid (32.2 mg, $0.121 \mathrm{mmol}, 73 \%$, $>99: 1 E: Z)$. $\mathbf{R}_{\mathbf{f}}$ (3:2 dichloromethane:ethyl acetate) $=0.32 .{ }^{1} \mathbf{H} \mathbf{~ N M R}\left(\mathrm{CDCl}_{3}, 400\right.$ MHz) $\delta 7.89-7.84(\mathrm{~d}, \mathrm{~J}=16.1 \mathrm{~Hz}, 1 \mathrm{H}), 7.46-7.43(\mathrm{dd}, \mathrm{J}=7.7,1.5 \mathrm{~Hz}, 1 \mathrm{H}), 7.31$ $-7.26(\mathrm{~m}, 1 \mathrm{H}), 6.93-6.86(\mathrm{~m}, 2 \mathrm{H}), 6.56-6.51(\mathrm{~d}, \mathrm{~J}=16.4 \mathrm{~Hz}, 1 \mathrm{H}), 6.08-5.94(\mathrm{~s}$, $1 \mathrm{H}), 3.85-3.82(\mathrm{~s}, 3 \mathrm{H}), 2.94-2.91(\mathrm{~d}, \mathrm{~J}=4.9 \mathrm{~Hz}, 3 \mathrm{H}) .{ }^{13} \mathbf{C ~ N M R}\left(\mathrm{CDCl}_{3}, 100\right.$ MHz) $\delta 166.2,158.2,136.7,130.6,128.2,124.6,121.6,112.8,57.7,27.0$. HRMS (ESI-TOF) m/z: [M+H] ${ }^{+}$ Calcd for $\mathrm{C}_{11} \mathrm{H}_{14} \mathrm{NO}_{2}{ }^{+}$192.1019; Found 192.1012.

\section{(E)-3-(4-(benzyloxy)phenyl)- $N$-methylacrylamide (2h)}<smiles>CNC(=O)/C=C/c1ccc(OCc2ccccc2)cc1</smiles>

Prepared according to Procedure 5.1. White solid (37.0 $\mathrm{mg}, 0.140 \mathrm{mmol}$, 92\%, >99:1 E:Z). $\mathbf{R}_{\mathbf{f}}(3: 2$ dichloromethane:ethyl acetate $)=0.44 .{ }^{1} \mathbf{H}$ NMR $\left(\mathrm{CDCl}_{3}, 400 \mathrm{MHz}\right) \delta 7.61-7.54$ (major, $\left.\mathrm{d}, \mathrm{J}=15.6 \mathrm{~Hz}, 1 \mathrm{H}\right), 7.45-7.30(\mathrm{~m}$, $7 \mathrm{H}), 6.95-6.89(\mathrm{~d}, \mathrm{~J}=8.7 \mathrm{~Hz}, 2 \mathrm{H}), 6.67-6.63($ minor, $\mathrm{d}, \mathrm{J}=12.6 \mathrm{~Hz}, 1 \mathrm{H})$, $6.36-6.24$ (major, d, J = 15.6 Hz, 1H), $6.08-5.95(\mathrm{~d}, \mathrm{~J}=5.5 \mathrm{~Hz}, 1 \mathrm{H}), 5.88$ - 5.84, (minor, d, J = 12.6 Hz, 1H), $5.07-5.04(\mathrm{~s}, 2 \mathrm{H}), 2.96-2.87(\mathrm{~d}, \mathrm{~J}=4.9 \mathrm{~Hz}, 3 \mathrm{H}) .{ }^{13} \mathbf{C} \mathbf{~ N M R}\left(\mathrm{CDCl}_{3}\right.$, $100 \mathrm{MHz}) \delta 167.0,160.0,140.2,136.5,129.3,128.6,128.1,127.8,127.5,118.5,115.1,70.0,26.5$. HRMS (ESI-TOF) m/z: $[\mathrm{M}+\mathrm{H}]^{+}$Calcd for $\mathrm{C}_{17} \mathrm{H}_{18} \mathrm{NO}_{2}{ }^{+}$268.1332; Found 268.1310.

\section{(E)- $N$-methyl-3-(m-tolyl)acrylamide (2i)}<smiles>CNC(=O)/C=C/c1cccc(C)c1</smiles>

Prepared according to Procedure 5.1. White solid (24.0 mg, $0.140 \mathrm{mmol}, 79 \%$, $>99: 1 E: Z) . \mathbf{R}_{\mathbf{f}}(3: 2$ dichloromethane:ethyl acetate $)=0.46 .{ }^{1} \mathbf{H} \mathbf{~ N M R}\left(\mathrm{CDCl}_{3}, 400\right.$ MHz) $\delta 7.61-7.55(\mathrm{~d}, \mathrm{~J}=15.6 \mathrm{~Hz}, 1 \mathrm{H}), 7.31-7.26(\mathrm{~m}, 2 \mathrm{H}), 7.25-7.19(\mathrm{t}, \mathrm{J}=$ $7.8 \mathrm{~Hz}, 1 \mathrm{H}), 7.16-7.12(\mathrm{~d}, \mathrm{~J}=8.0 \mathrm{~Hz}, 1 \mathrm{H}), 6.46-6.39(\mathrm{~d}, \mathrm{~J}=15.7 \mathrm{~Hz}, 1 \mathrm{H}), 6.13$ $-6.01(\mathrm{~s}, 1 \mathrm{H}), 2.96-2.91(\mathrm{~d}, \mathrm{~J}=2.9 \mathrm{~Hz}, 3 \mathrm{H}), 2.34-2.31(\mathrm{~s}, 3 \mathrm{H}) .{ }^{13} \mathbf{C} \mathbf{N M R}$ $\left(\mathrm{CDCl}_{3}, 100 \mathrm{MHz}\right) \delta 168.4,140.3,139.1,135.7,131.6,129.2,128.6,126.2,120.6,27.2,22.3$. HRMS (ESITOF) m/z: [M+H] ${ }^{+}$Calcd for $\mathrm{C}_{11} \mathrm{H}_{14} \mathrm{NO}^{+}$176.1070; Found 176.1055 .

\section{(E)-3-(4-(tert-butyl)phenyl)- $N$-methylacrylamide (2j)}<smiles>CNC(=O)/C=C/c1ccc(C(C)(C)C)cc1</smiles>

Prepared according to Procedure 5.1. White solid $(26.70 \mathrm{mg}, 0.123 \mathrm{mmol}$, $88 \%,>99: 1 E: Z) . \mathbf{R}_{\mathbf{f}}(3: 2$ dichloromethane:ethyl acetate $)=0.52 .{ }^{1} \mathbf{H} \mathbf{N M R}$ $\left(\mathrm{CDCl}_{3}, 400 \mathrm{MHz}\right) \delta 7.63-7.57(\mathrm{~d}, \mathrm{~J}=15.6 \mathrm{~Hz}, 1 \mathrm{H}), 7.45-7.40(\mathrm{~d}, \mathrm{~J}=8.3$ $\mathrm{Hz}, 2 \mathrm{H}), 7.38-7.32(\mathrm{~d}, \mathrm{~J}=8.5 \mathrm{~Hz}, 2 \mathrm{H}), 6.44-6.38(\mathrm{~d}, \mathrm{~J}=15.7 \mathrm{~Hz}, 1 \mathrm{H}), 6.09$ $-6.03(\mathrm{~d}, \mathrm{~J}=6.9,1 \mathrm{H}), 2.95-2.91(\mathrm{~d}, \mathrm{~J}=4.9 \mathrm{~Hz}, 3 \mathrm{H}), 1.32-1.29(\mathrm{~s}, 9 \mathrm{H}) .{ }^{13} \mathbf{C}$ NMR $\left(\mathrm{CDCl}_{3}, 126 \mathrm{MHz}\right) \delta 167.1,153.1,140.7,132.2,127.7,125.9,119.9$, 34.9, 32.3, 26.6. HRMS (ESI-TOF) m/z: $[\mathrm{M}+\mathrm{H}]^{+}$Calcd for $\mathrm{C}_{14} \mathrm{H}_{20} \mathrm{NO}^{+}$218.1539; Found 218.1534.

\section{(E)-3-(2-chlorophenyl)- $N$-methylacrylamide (2k)}<smiles>CNC(=O)/C=C/c1ccccc1Cl</smiles>

Prepared according to Procedure 5.1. White solid (49.9 mg, $0.255 \mathrm{mmol}, 88 \%$, $>99: 1$ E:Z). $\mathbf{R}_{\mathbf{f}}(3: 2$ dichloromethane:ethyl acetate $)=0.26 .{ }^{1} \mathbf{H} \mathbf{~ N M R}\left(\mathrm{CDCl}_{3}, 400\right.$ MHz) $\delta 7.98-7.93$, (major, d, J = $15.7 \mathrm{~Hz}, 1 \mathrm{H}), 7.54-7.50(\mathrm{dd}, \mathrm{J}=7.7,1.8 \mathrm{~Hz}$, $1 \mathrm{H}), 7.36-7.32(\mathrm{dd}, \mathrm{J}=8.0,1.3 \mathrm{~Hz}, 1 \mathrm{H}), 7.23=7.18(\mathrm{td}, \mathrm{J}=7.7,1.8 \mathrm{~Hz}, 1 \mathrm{H}), 7.18$ 
- $7.12(\mathrm{~m}, 1 \mathrm{H}), 6.71-6.64(\mathrm{~s}, 1 \mathrm{H}), 6.53-6.47$ (major, d, J = $15.7 \mathrm{~Hz}, 1 \mathrm{H}), 6.13-6.08$ (minor, d, J = 12.2 $\mathrm{Hz}, 1 \mathrm{H}), 2.94-2.91(\mathrm{~d}, \mathrm{~J}=4.9 \mathrm{~Hz}, 3 \mathrm{H}) .{ }^{13} \mathrm{C} \mathrm{NMR}\left(\mathrm{CDCl}_{3}, 100 \mathrm{MHz}\right) \delta 166.6,136.4,134.7,133.3,130.4$, 130.1 127.5, 127.0, 123.8, 26.6. HRMS (ESI-TOF) m/z: $[\mathrm{M}+\mathrm{H}]^{+}$Calcd for $\mathrm{C}_{10} \mathrm{H}_{11} \mathrm{ClNO}^{+}$196.0524; Found 196.0518 .

(E)- $N$-methyl-3-(4-(trifluoromethoxy)phenyl)acrylamide (2l)<smiles>CNC(=O)/C=C/c1ccc(OC(F)(F)F)cc1</smiles>

Prepared according to Procedure 5.1. White solid (19.2 mg, $0.0783 \mathrm{mmol}$, $68 \%,>99: 1 E: Z)$. $\mathbf{R}_{\mathbf{f}}(3: 2$ dichloromethane:ethyl acetate $)=0.52 .{ }^{1} \mathbf{H} \mathbf{~ N M R}$ (Acetone- $\left.d_{6}, 400 \mathrm{MHz}\right) \delta 7.72-7,68(\mathrm{~m}, 2 \mathrm{H}), 7.57-7.50$ (major, $\mathrm{d}, \mathrm{J}=$ $15.7 \mathrm{~Hz}, 1 \mathrm{H}), 7.37-7.29(\mathrm{~d}, \mathrm{~J}=7.9 \mathrm{~Hz}, 2 \mathrm{H}), 6.72-6.65$ (major, $\mathrm{d}, \mathrm{J}=$ $15.7 \mathrm{~Hz}, 1 \mathrm{H}), 6.10-6.06$ (minor, $\mathrm{d}, \mathrm{J}=12.7 \mathrm{~Hz}, 1 \mathrm{H}), 2.84-2.82(\mathrm{~d}, \mathrm{~J}=$

$4.70 \mathrm{~Hz}, 3 \mathrm{H}) .{ }^{19} \mathbf{F}$ NMR (Acetone- $\left.d_{6}, 376 \mathrm{MHz}\right) \delta-58.4 .{ }^{13} \mathbf{C}$ NMR (Acetone- $\left.d_{6}, 100 \mathrm{MHz}\right) \delta 166.2,150.3$ (q, $\mathrm{J}=1.8 \mathrm{~Hz}$ ), 138.2, 135.6, 130.2, 124.2, 122.7, 121.15 (q, J = $254 \mathrm{~Hz}), 26.3$. HRMS (ESI-TOF) m/z: $[\mathrm{M}+\mathrm{H}]^{+}$Calcd for $\mathrm{C}_{11} \mathrm{H}_{11} \mathrm{~F}_{3} \mathrm{NO}_{2}{ }^{+}$246.0736; Found 246.0722.

\section{(E)-3-(3,5-difluorophenyl)- $N$-methylacrylamide (2m)}<smiles>CNC(=O)/C=C/c1cc(F)cc(F)c1</smiles>

Prepared according to Procedure 5.1. White solid (23.6 mg, $0.120 \mathrm{mmol}, 71 \%$, $>99: 1 E: Z) . \mathbf{R}_{\mathbf{f}}(3: 2$ dichloromethane:ethyl acetate $)=0.46 .{ }^{1} \mathbf{H} \mathbf{~ N M R}\left(\mathrm{CDCl}_{3}, 400\right.$ MHz) $\delta 7.53-7.47(\mathrm{~d}, \mathrm{~J}=15.6 \mathrm{~Hz}, 1 \mathrm{H}), 7.00-6.93(\mathrm{~m}, 2 \mathrm{H}), 6.79-6.73(\mathrm{~m}$, $1 \mathrm{H}), 6.45-6.39(\mathrm{~d}, \mathrm{~J}=15.6 \mathrm{~Hz}, 1 \mathrm{H}), 6.15-6.08(\mathrm{~s}, 1 \mathrm{H}), 2.96-2.92(\mathrm{~d}, \mathrm{~J}=4.9$ $\mathrm{Hz}, 3 \mathrm{H}) .{ }^{19} \mathbf{F}$ NMR $\left(\mathrm{CDCl}_{3}, 376 \mathrm{MHz}\right) \delta-109.4(\mathrm{t}, \mathrm{J}=7.9 \mathrm{~Hz}) .{ }^{13} \mathbf{C ~ N M R}\left(\mathrm{CDCl}_{3}\right.$, $100 \mathrm{MHz}) \delta 165.8,164.4(\mathrm{~d}, \mathrm{~J}=12.9 \mathrm{~Hz}), 161.9(\mathrm{~d}, \mathrm{~J}=12.9 \mathrm{~Hz}), 138.4(\mathrm{t}, \mathrm{J}=$ $2.6 \mathrm{~Hz}), 138.1(5, \mathrm{~J}=9.5 \mathrm{~Hz}), 123.2,110.8(\mathrm{~m}), 104.7(\mathrm{t}, \mathrm{J}=25.5 \mathrm{~Hz}), 26.6$. HRMS (ESI-TOF) m/z: $[\mathrm{M}+\mathrm{H}]^{+}$Calcd for $\mathrm{C}_{10} \mathrm{H}_{10} \mathrm{~F}_{2} \mathrm{NO}^{+}$198.0725; Found 198.0723.

(E)-3-(4-cyanophenyl)- $N$-methylacrylamide (2n)<smiles>CNC(=O)/C=C/c1ccc(C#N)cc1</smiles>

Prepared according to Procedure 5.1. White solid $(21.0 \mathrm{mg}, 0.110 \mathrm{mmol}$, 86\%, 94:6 E:Z). $\mathbf{R}_{\mathbf{f}}(3: 2$ dichloromethane:ethyl acetate $)=0.08 .{ }^{1} \mathbf{H}$ NMR $\left(\mathrm{CD}_{3} \mathrm{OD}, 400 \mathrm{MHz}\right) \delta 7.73-7.64$ (major, $\left.\mathrm{m}, 4 \mathrm{H}\right), 7.64-7.57$ (minor, $\mathrm{m}, 4 \mathrm{H}$ ), $7.55-7.50$ (major, d, J = 15.8 Hz, 1H), $6.79-6.75$ (minor, d, J = 12.4 Hz, 1H), $6.69-6.63$ (major, d, J = 16.2 Hz, 1H), $6.16-6.12$ (minor, d, J = 12.6 $\mathrm{Hz}, 1 \mathrm{H}), 2.87-2.84(\mathrm{~s}, 3 \mathrm{H}) .{ }^{13} \mathbf{C}$ NMR $\left(\mathrm{CD}_{3} \mathrm{OD}, 100 \mathrm{MHz}\right) \delta 167.9,140.6,139.0,133.4,129.1,125.1$, 119.2, 113.3, 26.6. HRMS (ESI-TOF) m/z: $[\mathrm{M}+\mathrm{H}]^{+}$Calcd for $\mathrm{C}_{11} \mathrm{H}_{11} \mathrm{~N}_{2} \mathrm{O}^{+}$187.0866; Found 187.0851.

\section{(E)- $N$-methyl-3-(thiophen-3-yl)acrylamide (2o)}

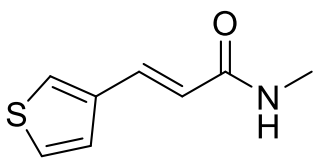

Prepared according to Procedure 5.1. White solid $(35.3 \mathrm{mg}, 0.211 \mathrm{mmol}, 90 \%$, $>99: 1 E: Z) . \mathbf{R}_{\mathbf{f}}(3: 2$ dichloromethane:ethyl acetate $)=0.43 .{ }^{1} \mathbf{H} \mathbf{~ N M R}\left(\mathrm{CDCl}_{3}, 400\right.$ $\mathrm{MHz}$ ) $\delta 7.64-7.57$ (major, d, J = 16.2 Hz, 1H), $7.41-7.37$ (dd, J = 2.9, $1.3 \mathrm{~Hz}$, 2H), $7.29-7.25$ (ddd, J = 5.7, 2.6, 0.7, 1H), $7.24-7.21(\mathrm{~m}, 1 \mathrm{H}), 6.69-6.65$ (minor, $\mathrm{d}, \mathrm{J}=12.7 \mathrm{~Hz}, 1 \mathrm{H}$ ), $6.34-6.26$ (major, d, J = 15.6 Hz, 1HJ), $6.34-6.26$ (s, 1H), $5.87-5.83$ (minor, d, J $=12.5 \mathrm{~Hz}, 1 \mathrm{H}), 2.94-2.91(\mathrm{~d}, \mathrm{~J}=4.9 \mathrm{~Hz}, 3 \mathrm{H}) .{ }^{13} \mathbf{C ~ N M R}\left(\mathrm{CDCl}_{3}, 100 \mathrm{MHz}\right) \delta 167.2,137.9,134.3,127.0$, 126.8, 125.1, 120.6, 26.6. HRMS (ESI-TOF) $\mathrm{m} / \mathrm{z}$ : $[\mathrm{M}+\mathrm{H}]^{+}$Calcd for $\mathrm{C}_{8} \mathrm{H}_{10} \mathrm{NOS}^{+}$168.0478; Found 168.0473. 
(E)-N-methyl-3-(pyridin-3-yl)acrylamide (2p)<smiles>CNC(=O)/C=C/c1cccnc1</smiles>

Prepared according to Procedure 5.1. White solid (43.3 mg, $0.267 \mathrm{mmol}, 88 \%$, $>99: 1 E: Z) . \mathbf{R}_{\mathbf{f}}(3: 2$ dichloromethane:ethyl acetate $)=0.22 .{ }^{1} \mathbf{H}$ NMR $\left(\mathrm{CD}_{3} \mathrm{OD}, 400\right.$ $\mathrm{MHz}) \delta 8.72-8.67(\mathrm{~d}, \mathrm{~J}=2.3 \mathrm{~Hz}, 1 \mathrm{H}), 8.53-8.48(\mathrm{dd}, \mathrm{J}=4.9,1.6 \mathrm{~Hz}, 1 \mathrm{H}), 8.05-$ $8.00(\mathrm{dt}, \mathrm{J}=7.4,2.0 \mathrm{~Hz}, 1 \mathrm{H}), 7.57-7.50(\mathrm{~d}, \mathrm{~J}=15.9 \mathrm{~Hz}, 1 \mathrm{H}), 7.48-7.43$ (ddd, $\mathrm{J}=$ 8.0, 4.9, 0.9 Hz, 1H), $6.73-6.67(\mathrm{~d}, \mathrm{~J}=15.9 \mathrm{~Hz}, 1 \mathrm{H}), 6.70-6.68(\mathrm{~d}, \mathrm{~J}=2.1 \mathrm{~Hz}$, $1 \mathrm{H}), 2.87-2.84(\mathrm{~s}, 3 \mathrm{H}) .{ }^{13} \mathrm{C}$ NMR $\left(\mathrm{CD}_{3} \mathrm{OD}, 100 \mathrm{MHz}\right) \delta 168.3,150.7,149.7,137.3,136.2,132.8,115.5$, 124.6, 124.5, 26.5. HRMS (ESI-TOF) m/z: $[\mathrm{M}+\mathrm{H}]^{+}$Calcd for $\mathrm{C}_{9} \mathrm{H}_{11} \mathrm{~N}_{2} \mathrm{O}^{+}$163.0866; Found 163.0858.

\section{(E)-3-(cyclohex-1-en-1-yl)- $N$-methylacrylamide (4a)}<smiles>CNC(=O)/C=C/C1=CCCCC1</smiles>

Prepared according to Procedure 5.1. White solid (55.9 mg, $0.338 \mathrm{mmol}, 91 \%$, $>99: 1 E: Z) . \mathbf{R}_{\mathbf{f}}(3: 2$ dichloromethane:ethyl acetate $)=0.31 .{ }^{1} \mathbf{H} \mathbf{~ N M R}\left(\mathrm{CDCl}_{3}, 400\right.$ MHz) $\delta 7.22-7.16(\mathrm{~d}, \mathrm{~J}=15.4 \mathrm{~Hz}, 1 \mathrm{H}), 6.12-6.08(\mathrm{t}, \mathrm{J}=4.1 \mathrm{~Hz}, 1 \mathrm{H}), 5.74-5.67$ $(\mathrm{d}, \mathrm{J}=15.5 \mathrm{~Hz}, 1 \mathrm{H}), 5.65-5.57(\mathrm{~s}, 1 \mathrm{H}), 2.91-2.86(\mathrm{~d}, \mathrm{~J}=4.90 \mathrm{~Hz}, 1 \mathrm{H}), 2.22-$ $2.14(\mathrm{~m}, 2 \mathrm{H}), 2.14-2.07(\mathrm{~m}, 2 \mathrm{H}), 1.72-1.64(\mathrm{~m}, 2 \mathrm{H}), 1.64-1.55(\mathrm{~m}, 2 \mathrm{H}) .{ }^{13} \mathrm{C}$ NMR $\left(\mathrm{CDCl}_{3}, 100 \mathrm{MHz}\right) \delta 167.6,144.3,137.3,134.7,117.0,26.5,26.4,24.4,22.3,22.3$. HRMS (ESITOF) $\mathrm{m} / \mathrm{z}:[\mathrm{M}+\mathrm{H}]^{+}$Calcd for $\mathrm{C}_{10} \mathrm{H}_{16} \mathrm{NO}^{+}$166.1226; Found 166.1223.

\section{(E)-3-cyclohexyl- $N$-methylacrylamide (4b)}<smiles>CNC(=O)/C=C/C1CCCCC1</smiles>

Prepared according to Procedure 5.2. White solid (51.3 mg, $0.307 \mathrm{mmol}$, 62\%, 81:19 E:Z). $\mathbf{R}_{\mathbf{f}}(3: 2$ dichloromethane:ethyl acetate $)=0.41 .{ }^{1} \mathbf{H} \mathbf{~ N M R}\left(\mathrm{CDCl}_{3}, 400\right.$ MHz) $\delta 6.77-6.70$ (major, dd, J = 15.5, $6.8 \mathrm{~Hz}, 1 \mathrm{H}), 5.97-5.89(\mathrm{~s}, 1 \mathrm{H}), * 5.85-$ $5.75(\mathrm{~s}, 1 \mathrm{H}), 5.78-5.72$ (minor, dd, $\mathrm{J}=11.7,9.9 \mathrm{~Hz}, 1 \mathrm{H}), 5.74-5.68$ (major, dd, J $=15.5,1.4 \mathrm{~Hz}, 1 \mathrm{H}), 5.57-5.54($ minor $, \mathrm{dd}, \mathrm{J}=11.5,0.9 \mathrm{~Hz}, 1 \mathrm{H}), 2.85-2.82(\mathrm{~d}, \mathrm{~J}$ $=4.9 \mathrm{~Hz}, 3 \mathrm{H}), 2.82-2.80$ (minor, $\mathrm{d}, \mathrm{J}=4.9 \mathrm{~Hz}, 3 \mathrm{H}), * 2.78-2.75(\mathrm{~d}, \mathrm{~J}=4.8 \mathrm{~Hz}, 3 \mathrm{H}), 2.19-2.12(\mathrm{~m}, 2 \mathrm{H})$, $2.11-2.01(\mathrm{~m}, 1 \mathrm{H}), 1.76-1.57(\mathrm{~m}, 13 \mathrm{H}), 1.53-1.44(\mathrm{~m}, 2 \mathrm{H}), 1.38-0.97(\mathrm{~m}, 12 \mathrm{H}), 0.91-0.79(\mathrm{~m}, 2 \mathrm{H})$. ${ }^{13} \mathrm{C} \mathrm{NMR}\left(\mathrm{CDCl}_{3}, 100 \mathrm{MHz}\right) \delta 174.3, * 167.3,151.0$ (minor), 149.5, 121.2, 120.1 (minor), 40.3, 27.5, 27.0, $34.2,33.3,33.2,32.8,32.1,26.6,26.4,26.3,26.1,25.9,25.6$. HRMS (ESI-TOF) m/z: $[\mathrm{M}+\mathrm{H}]^{+}$Calcd for $\mathrm{C}_{10} \mathrm{H}_{18} \mathrm{NO}^{+}$168.1383; Found 168.1381.

\section{(E)-7-(benzyloxy)- $N$-methylhept-2-enamide (4c)}<smiles>CNC(=O)/C=C/CCCCOCc1ccccc1</smiles>

Prepared according to Procedure 5.2. White solid (42.5 mg, 0.172 mmol, 70\%, 83:17 E:Z). $\mathbf{R}_{\mathbf{f}}(3: 2$ dichloromethane:ethyl acetate $)=$ 0.29. ${ }^{1} \mathbf{H}$ NMR $\left(\mathrm{CDCl}_{3}, 400 \mathrm{MHz}\right) \delta 7.37-7.29(\mathrm{~m}, 7 \mathrm{H}), 7.30-$ 7.24 (m, 2H), $6.84-6.75$ (major, dt, J = 16.0, $6.7 \mathrm{~Hz}, 1 \mathrm{H}), 5.99-$ 5.91 (minor, dt, $\mathrm{J}=11.5,7.5 \mathrm{~Hz}, 1 \mathrm{H}$ ), $5.78-5.71$ (major, dt, $\mathrm{J}=$ 15.2, 1.4 Hz, 1H), $5.69-5.65$ (minor, dt, J = 11.6, $1.7 \mathrm{~Hz}, 1 \mathrm{H}), 5.67-5.51(\mathrm{~s}, 1 \mathrm{H}), 4.49-4.47$ (s, 2H), $* 4.49-4.47(\mathrm{~s}, 2 \mathrm{H}), 3.48-3.43(\mathrm{t}, \mathrm{J}=7.0 \mathrm{~Hz}, 2 \mathrm{H}), * 3.48-3.43(\mathrm{t}, \mathrm{J}=7.1 \mathrm{~Hz}, 2 \mathrm{H}), 2.86-2.83$ (major, d, $\mathrm{J}=4.9 \mathrm{~Hz}, 3 \mathrm{H}$ ), $2.82-2.80$ (minor, $\mathrm{d}, \mathrm{J}=4.9 \mathrm{~Hz}, 3 \mathrm{H}$ ), *2.79-2.76 (major, $\mathrm{d}, \mathrm{J}=4.8 \mathrm{~Hz}, 3 \mathrm{H}), 2.21-2.11$ $(\mathrm{m}, 2 \mathrm{H}), * 2.21-2.11(\mathrm{~m}, 2 \mathrm{H}), 1.70-1.58(\mathrm{~m}, 2 \mathrm{H}), * 1.70-1.58(\mathrm{~m}, 2 \mathrm{H}), 1.58-1.48(\mathrm{~m}, 2 \mathrm{H}), * 1.58-$ $1.48(\mathrm{~m}, 2 \mathrm{H}), 1.42-1.27(\mathrm{~m}, 2 \mathrm{H}), * 1.42-1.27(\mathrm{~m}, 2 \mathrm{H}) .{ }^{13} \mathbf{C} \mathbf{N M R}\left(\mathrm{CDCl}_{3}, 100 \mathrm{MHz}\right) \delta * 173.8,166.8$, 145.2 (minor), 144.2, 138.7, 128.5, *128.5, 127.7, *127.6, 127.6 (minor), 123.8, 73.0, *73.0, *70.4, 70.1, 36.7, 31.8, 29.8 (minor), *29.7, 29.4 (minor), 29.3, 29.2, 28.5 (minor), 26.4, *26.0, *25.9, 25.0. HRMS (ESI-TOF) $\mathrm{m} / \mathrm{z}:[\mathrm{M}+\mathrm{H}]^{+}$Calcd for $\mathrm{C}_{15} \mathrm{H}_{22} \mathrm{NO}_{2}{ }^{+}$248.1645; Found 248.1637. 


\section{(E)-3-cyclopropyl- $N$-methylacrylamide (4d)}

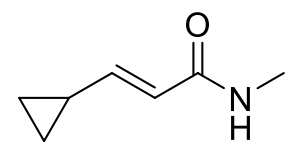

Prepared according to Procedure 5.1. Clear oil (28.9 mg, 0.231 mmol, 71\%, >99:1

$E: Z) . \mathbf{R}_{\mathbf{f}}(3: 2$ dichloromethane:ethyl acetate $)=0.29 .{ }^{1} \mathbf{H} \mathbf{~ N M R}\left(\mathrm{CDCl}_{3}, 400 \mathrm{MHz}\right) \delta$ $6.33-6.25$ (major, dd, J = 15.0, $10.0 \mathrm{~Hz}, 1 \mathrm{H}), 5.86-5.81$ (major, d, J = $14.9 \mathrm{~Hz}, 1 \mathrm{H}$ ), $5.62-5.51(\mathrm{~s}, 1 \mathrm{H}), 5.25-5.19($ minor, $\mathrm{t}, \mathrm{J}=10.8 \mathrm{~Hz}, 1 \mathrm{H}), 2.86-2.83(\mathrm{~d}, \mathrm{~J}=4.9 \mathrm{~Hz}$, $3 \mathrm{H}), 1.55-1.45(\mathrm{~m}, 1 \mathrm{H}), 0.90-0.83(\mathrm{~m}, 2 \mathrm{H}), 0.60-0.55(\mathrm{~m}, 2 \mathrm{H}) .{ }^{13} \mathbf{C} \mathbf{N M R}\left(\mathrm{CDCl}_{3}, 100 \mathrm{MHz}\right) \delta 166.9$, 148.6, 120.5, 26.4, 15.8, 8.3. HRMS (ESI-TOF) m/z: $[\mathrm{M}+\mathrm{H}]^{+}$Calcd for $\mathrm{C}_{7} \mathrm{H}_{12} \mathrm{NO}^{+}$126.0913; Found 126.0912 .

\section{$N$-methylacrylamide (4e)}<smiles>C=CC(=O)NC</smiles>

Prepared according to Procedure 5.1 with the exception that pinacolborane was added first followed by addition of base. Clear oil (50.1 mg, $0.862 \mathrm{mmol}, 68 \%$,). $\mathbf{R}_{\mathbf{f}}(3: 2$ dichloromethane:ethyl acetate $)=0.24 .{ }^{1} \mathbf{H}$ NMR $\left(\mathrm{CDCl}_{3}, 400 \mathrm{MHz}\right) \delta 6.30-6.23(\mathrm{dd}, \mathrm{J}=$ $17.0,1.5 \mathrm{~Hz}, 1 \mathrm{H}), 6.13-6.05(\mathrm{dd}, \mathrm{J}=17.0,10.3 \mathrm{~Hz}, 1 \mathrm{H}), 5.83-5.66(\mathrm{~s}, 1 \mathrm{H}), 5.65-5.59$ $(\mathrm{dd}, \mathrm{J}=10.2,1.5 \mathrm{~Hz}, 1 \mathrm{H}), 2.90-2.87(\mathrm{~d}, \mathrm{~J}=4.9 \mathrm{~Hz}, 3 \mathrm{H}) .{ }^{13} \mathbf{C ~ N M R}\left(\mathrm{CDCl}_{3}, 100 \mathrm{MHz}\right) \delta 164.3,130.0$, 126.3, 26.5, 25.0. HRMS (ESI-TOF) m/z: $[\mathrm{M}+\mathrm{H}]^{+}$Calcd for $\mathrm{C}_{4} \mathrm{H}_{8} \mathrm{NO}^{+} 86.0600$; Found 86.0600.

\section{(E)-3-(dimethyl(phenyl)silyl)- $N$-methylacrylamide (4f)}<smiles>CNC(=O)/C=C/[Si](C)(C)c1ccccc1</smiles>

Prepared according to Procedure 5.1 with the exception that pinacolborane was added first followed by addition of base. Pale yellow oil $(43.7 \mathrm{mg}, 0.250 \mathrm{mmol}$, $80 \%,>99: 1 \quad E: Z) . \mathbf{R}_{\mathbf{f}}(3: 2$ dichloromethane:ethyl acetate $)=0.55 .{ }^{1} \mathbf{H} \mathbf{~ N M R}$ $\left(\mathrm{CDCl}_{3}, 400 \mathrm{MHz}\right) \delta 7.55-7.47(\mathrm{~m}, 2 \mathrm{H}), 7.40-7.31(\mathrm{~m}, 3 \mathrm{H}), 7.19-7.11(\mathrm{~d}, \mathrm{~J}$ $=18.5 \mathrm{~Hz}, 1 \mathrm{H}), 6.23-6.16(\mathrm{~d}, \mathrm{~J}=18.5 \mathrm{~Hz}, 1 \mathrm{H}), 5.76-5.66(\mathrm{~s}, 1 \mathrm{H}), 2.88-2.85(\mathrm{~d}, \mathrm{~J}=4.9 \mathrm{~Hz}, 3 \mathrm{H}), 0.40$ $-0.38(\mathrm{~s}, 6 \mathrm{H}) .{ }^{13} \mathrm{C} \mathrm{NMR}\left(\mathrm{CDCl}_{3}, 100 \mathrm{MHz}\right) \delta 166.1,141.7,138.1,136.9,134.0,129.5,128.1,26.5,-2.9$. HRMS (ESI-TOF) m/z: $[\mathrm{M}+\mathrm{H}]^{+}$Calcd for $\mathrm{C}_{12} \mathrm{H}_{18} \mathrm{NOSi}^{+}$220.1152; Found 220.1142.

\section{(E)-cinnamamide (6a)}

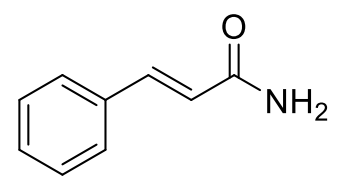

Prepared according to Procedure 5.2. White solid (21.5 mg, $0.146 \mathrm{mmol}, 70 \%$, $>99: 1 \mathrm{E:Z})$. $\mathbf{R}_{\mathbf{f}}(3: 2$ dichloromethane:ethyl acetate $)=0.27 .{ }^{1} \mathbf{H} \mathbf{~ N M R}\left(\mathrm{CDCl}_{3}, 400\right.$ MHz) $\delta 7.68-7.62$ (major, d, J = $15.7 \mathrm{~Hz}, 1 \mathrm{H}), 7.54-7.49(\mathrm{~m}, 2 \mathrm{H}), 7.40-7.35$ (m, 3H), $6.88-6.83$ (minor, d, J = 12.5 Hz, 1H), $6.51-6.43$ (major, d, J = 15.7 Hz, $1 \mathrm{H}), 6.01-5.97$ (minor, $\mathrm{d}, \mathrm{J}=12.5 \mathrm{~Hz}, 1 \mathrm{H}) .{ }^{13} \mathbf{C ~ N M R}\left(\mathrm{CDCl}_{3}, 100 \mathrm{MHz}\right) \delta 168.5$, 142.0, 135.8, 130.1, 129.0, 128.5, 120.8. HRMS (ESI-TOF) m/z: $[\mathrm{M}+\mathrm{H}]^{+}$Calcd for $\mathrm{C}_{9} \mathrm{H}_{10} \mathrm{NO}^{+}$148.0757; Found 148.0738.

2.8 mmol scale: White solid (372 mg, $2.53 \mathrm{mmol}, 92 \%,>99: 1 \mathrm{E:Z})$

\section{Procedure for $2.8 \mathrm{mmol}$ scale:}

A flame-dried $25 \mathrm{~mL}$ round bottom flask was charged with dry propiolamide (402 $\mathrm{mg}, 2.8 \mathrm{mmol}$ ) and placed under $\mathrm{N}_{2}$ via Schlenk technique. The reaction was diluted with THF (13.0 mL) and stirred at room temperature. $\mathrm{KO} t \mathrm{Bu}(310 \mathrm{mg}, 2.8 \mathrm{mmol})$ was added as a solid. Pinacolborane $(0.44 \mathrm{~mL}, 3.0 \mathrm{mmol})$ was then slowly added dropwise (1 drop every 3-4 seconds). The reaction was stirred at room temperature for 10 minutes. The reaction mixture was concentrated in vacuo and the resulting crude residue was purified by silica gel column chromatography (eluted with a 30-40\% gradient ethyl acetate in dichloromethane) to afford the product as a white solid. 


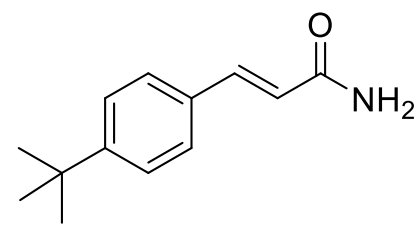

Prepared according to Procedure 5.2. White solid (23.1 mg, $0.190 \mathrm{mmol}$, 60\%, 97:3 E:Z). $\mathbf{R}_{\mathbf{f}}$ (3:2 dichloromethane:ethyl acetate) $=0.38 .{ }^{1} \mathbf{H}$ NMR $\left(\mathrm{CD}_{3} \mathrm{OD}, 400 \mathrm{MHz}\right) \delta 7.56-7.50($ major, $\mathrm{d}, \mathrm{J}=15.8 \mathrm{~Hz}, 1 \mathrm{H}), 7.50-7.46(\mathrm{~m}$, 2H), $7.44-7.39(\mathrm{~m}, 2 \mathrm{H}), 6.73-6.69$ (minor, d, J = 12.7 Hz, 1H), $6.63-6.57$ (major, d, J = 15.8 Hz, 1H), $5.99-5.95$ (minor, d, J = $12.7 \mathrm{~Hz}, 1 \mathrm{H}$ ), $1.33-$ 1.29 (s, 9H). ${ }^{13} \mathrm{C}$ NMR $\left(\mathrm{CD}_{3} \mathrm{OD}, 100 \mathrm{MHz}\right) \delta 171.2,154.5,142.6,133.4$, 128.8, 126.9, 120.5, 35.7, 31.6. HRMS (ESI-TOF) m/z: $[\mathrm{M}+\mathrm{H}]^{+}$Calcd for $\mathrm{C}_{13} \mathrm{H}_{18} \mathrm{NO}^{+}$204.1383; Found 204.1383.

\section{(E)-3-(4-(trifluoromethyl)phenyl)acrylamide (6c)}<smiles>NC(=O)/C=C/c1ccc(C(F)(F)F)cc1</smiles>

Prepared according to Procedure 5.2. White solid (30.3 mg, $0.141 \mathrm{mmol}$, $62 \%,>99: 1 E: Z) . \mathbf{R}_{\mathbf{f}}(3: 2$ dichloromethane:ethyl acetate $)=0.33 .{ }^{1} \mathbf{H}$ NMR $\left(\mathrm{CD}_{3} \mathrm{OD}, 400 \mathrm{MHz}\right) \delta 7.76-7.71(\mathrm{~d}, \mathrm{~J}=8.2 \mathrm{~Hz}, 2 \mathrm{H}), 7.71-7.64(\mathrm{~d}, \mathrm{~J}=8.3$ $\mathrm{Hz}, 2 \mathrm{H}$ ), $7.62-7.55$ (major, d, J = $15.9 \mathrm{~Hz}, 1 \mathrm{H}$ ), $6.78-6.72$ (major, d, J = $15.9 \mathrm{~Hz}, 1 \mathrm{H}), 6.20-6.16$ (minor, $\mathrm{d}, \mathrm{J}=12.6 \mathrm{~Hz}, 1 \mathrm{H}) .{ }^{19} \mathbf{F}$ NMR $\left(\mathrm{CD}_{3} \mathrm{OD}\right.$, $376 \mathrm{MHz}) \delta-64.3 .{ }^{13} \mathbf{C}$ NMR $\left(\mathrm{CD}_{3} \mathrm{OD}, 100 \mathrm{MHz}\right) \delta 170.2,140.7,140.0,132.1(\mathrm{q}, \mathrm{J}=32.6 \mathrm{~Hz}), 129.3$, $126.8(\mathrm{q}, \mathrm{J}=3.9 \mathrm{~Hz}), 125.6(\mathrm{q}, \mathrm{J}=269 \mathrm{~Hz}), 124.4$, 124.1. HRMS (ESI-TOF) $\mathrm{m} / \mathrm{z}:[\mathrm{M}+\mathrm{H}]^{+}$Calcd for $\mathrm{C}_{10} \mathrm{H}_{9} \mathrm{~F}_{3} \mathrm{NO}^{+} 216.0631$; Found 216.0631 .

\section{(E)-3-(4-(trifluoromethoxy)phenyl)acrylamide (6d)}<smiles>NC(=O)/C=C/c1ccc(OC(F)(F)F)cc1</smiles>

Prepared according to Procedure 5.2. White solid (20.5 mg, $0.0887 \mathrm{mmol}$, 78\%, >99:1 E:Z). $\mathbf{R}_{\mathbf{f}}(3: 2$ dichloromethane:ethyl acetate $)=0.34 .{ }^{1} \mathbf{H} \mathbf{~ N M R}$ (Acetone- $\left.d_{6}, 400 \mathrm{MHz}\right) \delta 7.74-7.69(\mathrm{~m}, 2 \mathrm{H}), 7.59-7.53$ (major, $\mathrm{d}, \mathrm{J}=$ $15.8 \mathrm{~Hz}, 1 \mathrm{H}), 7.39-7.33(\mathrm{~m}, 2 \mathrm{H}), 7.10-7.00(\mathrm{~s}, 1 \mathrm{H}), 6.78-6.72$ (major, $\mathrm{d}, \mathrm{J}=15.9 \mathrm{~Hz}, 1 \mathrm{H}), 6.62-6.50(\mathrm{~s}, 1 \mathrm{H}), 6.16-6.11$ (minor, $\mathrm{d}, \mathrm{J}=12.8 \mathrm{~Hz}$, $1 \mathrm{H}) .{ }^{19} \mathbf{F}$ NMR (Acetone- $\left.d_{6}, 376 \mathrm{MHz}\right) \delta-58.6 .{ }^{13} \mathbf{C}$ NMR (Acetone- $\left.d_{6}, 100 \mathrm{MHz}\right) \delta 167.4,150.4(\mathrm{q}, \mathrm{J}=$ $1.8 \mathrm{~Hz}$ ), 138.2, 135.4, 130.3, 123.9, 122.2, 121.36 (q, J = $255 \mathrm{~Hz}$ ). HRMS (ESI-TOF) m/z: $[\mathrm{M}+\mathrm{H}]^{+}$Calcd for $\mathrm{C}_{10} \mathrm{H}_{9} \mathrm{~F}_{3} \mathrm{NO}_{2}{ }^{+}$232.0580; Found 232.0573.

\section{(E)-3-(4-nitrophenyl)acrylamide (6e)}<smiles>NC(=O)/C=C/c1ccc([N+](=O)[O-])cc1</smiles>

Prepared according to Procedure 5.2. Light yellow solid (10.6 mg, 0.0552 mmol, 52\%, 95:5 E:Z). $\mathbf{R}_{\mathbf{f}}(95: 5$ dichloromethane:methanol) $=0.28$. Purified using a 1-2\% gradient of methanol in dichloromethane. ${ }^{1} \mathbf{H}$ NMR $\left(\mathrm{CD}_{3} \mathrm{OD}\right.$, $400 \mathrm{MHz}$ ) $\delta 8.29$ - 8.24 (major, m, 2H), 8.20 - 8.17 (minor, $\mathrm{m}, 2 \mathrm{H}), 7.82$ 7.78 (major, $\mathrm{m}, 2 \mathrm{H}$ ), $7.75-7.71$ (minor, $\mathrm{m}, 2 \mathrm{H}), 7.65-7.59$ (major, $\mathrm{d}, \mathrm{J}=$ $15.9 \mathrm{~Hz}, 1 \mathrm{H}$ ), $6.90-6.86$ (minor, d, J = $12.6 \mathrm{~Hz}, 1 \mathrm{H}$ ), $6.84-6.78$ (major, d, J = 15.9 Hz, 1H), $6.27-6.23$ (minor, d, J = $12.6 \mathrm{~Hz}, 1 \mathrm{H}) .{ }^{13} \mathbf{C} \mathbf{N M R}\left(\mathrm{CD}_{3} \mathrm{OD}, 126 \mathrm{MHz}\right) \delta 169.8,149.7,142.6,139.9,136.4$ (minor), 131.2 (minor), 129.8, 125.9, 125.1, 124.2 (minor). HRMS (ESI-TOF) m/z: $[\mathrm{M}+\mathrm{H}]^{+}$Calcd for $\mathrm{C}_{9} \mathrm{H}_{9} \mathrm{~N}_{2} \mathrm{O}_{3}{ }^{+}$ 193.0608; Found 193.0596. 
(E)-3-(5-methylthiophen-2-yl)acrylamide (6f)

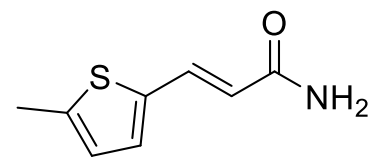

Prepared according to Procedure 5.2. White solid (14.2 mg, $0.0849 \mathrm{mmol}, 74 \%$, 98:2 E:Z). $\mathbf{R}_{\mathbf{f}}(3: 2$ dichloromethane:ethyl acetate $)=0.35 .{ }^{1} \mathbf{H} \mathbf{~ N M R}\left(\mathrm{CD}_{3} \mathrm{OD}, 400\right.$ $\mathrm{MHz}) \delta 7.61-7.54$ (major, d, J = $15.5 \mathrm{~Hz}, 1 \mathrm{H}), 7.08-7.06(\mathrm{~d}, \mathrm{~J}=3.6 \mathrm{~Hz}, 1 \mathrm{H})$, $6.89-6.85$ (minor, d, J = 13.1 Hz, 1H), $6.76-6.73(\mathrm{~m}, 1 \mathrm{H}), 6.31-6.22$ (major, $\mathrm{d}, \mathrm{J}=15.5 \mathrm{~Hz}, 1 \mathrm{H}), 5.73-5.69$ (minor, $\mathrm{d}, \mathrm{J}=12.4 \mathrm{~Hz}, 1 \mathrm{H}), 2.49-2.47(\mathrm{~s}, 3 \mathrm{H}) .{ }^{13} \mathbf{C}$ NMR $\left(\mathrm{CD}_{3} \mathrm{OD}, 100\right.$ MHz) $\delta 170.9,144.5,139.2,135.8,132.4,127.6,118.7,15.5$. HRMS (ESI-TOF) $\mathrm{m} / \mathrm{z}:[\mathrm{M}+\mathrm{H}]^{+}$Calcd for $\mathrm{C}_{8} \mathrm{H}_{10} \mathrm{NOS}^{+}$168.0478; Found 168.0477.

\section{(E)-3-(pyridin-2-yl)acrylamide (6g)}<smiles>NC(=O)/C=C/c1ccccn1</smiles>

Prepared according to Procedure 5.2. White solid (28.7 mg, $0.194 \mathrm{mmol}, 80 \%$, $>99: 1 E: Z) . \mathbf{R}_{\mathbf{f}}(95: 5$ dichloromethane:methanol) $=0.13$. Purified using a 4-6\% gradient methanol in dichloromethane. ${ }^{1} \mathbf{H}$ NMR $\left(\mathrm{CD}_{3} \mathrm{OD}, 400 \mathrm{MHz}\right) \delta 8.58-8.56$ $(\mathrm{ddd}, \mathrm{J}=4.9,1.8,0.9 \mathrm{~Hz}, 1 \mathrm{H}), 7.87-7.81(\mathrm{td}, \mathrm{J}=7.7,1.8 \mathrm{~Hz}, 1 \mathrm{H}), 7.62-7.58(\mathrm{dt}$, $\mathrm{J}=7.9,1.0 \mathrm{~Hz}, 1 \mathrm{H}), 7.58-7.54(\mathrm{~d}, \mathrm{~J}=15.7 \mathrm{~Hz}, 7.39-7.35(\mathrm{ddd}, \mathrm{J}=7.6,4.9,1.1$ $\mathrm{Hz}, 1 \mathrm{H}), 7.03-6.98(\mathrm{~d}, \mathrm{~J}=15.6 \mathrm{~Hz}, 1 \mathrm{H}), 7.01-6.99(\mathrm{t}, \mathrm{J}=2.1 \mathrm{~Hz}, 2 \mathrm{H}) .{ }^{13} \mathbf{C} \mathbf{N M R}\left(\mathrm{CD}_{3} \mathrm{OD}, 100 \mathrm{MHz}\right) \delta$ 170.2, 154.6, 150.8, 141.0, 138.8, 126.1, 126.0, 125.5, 125.2. HRMS (ESI-TOF) $\mathrm{m} / \mathrm{z}:[\mathrm{M}+\mathrm{H}]^{+}$Calcd for $\mathrm{C}_{8} \mathrm{H}_{9} \mathrm{~N}_{2} \mathrm{O}^{+}$149.0709; Found 149.0705.

\section{(E)-3-(pyridin-3-yl)acrylamide (6h)}<smiles>NC(=O)/C=C/c1cccnc1</smiles>

Prepared according to Procedure 5.2. White solid (29.2 mg, $0.197 \mathrm{mmol}, 99 \%$, $>99: 1 \mathrm{E:Z}$ ). $\mathbf{R}_{\mathbf{f}}(95: 5$ dichloromethane:methanol) $=0.11$. Purified using a 4-6\% gradient methanol in dichloromethane. ${ }^{1} \mathbf{H}$ NMR $\left(\mathrm{CD}_{3} \mathrm{OD}, 400 \mathrm{MHz}\right) \delta 8.74-8.69$ $(\mathrm{s}, 1 \mathrm{H}), 8.55-8.49(\mathrm{~d}, \mathrm{~J}=4.9 \mathrm{~Hz}, 1 \mathrm{H}), 8.07-8.03$ (dddd, J = 8.0, 2.2, 1.6, 0.5 Hz, $1 \mathrm{H}$ ), $7.60-7.54$ (major, d, J = $16.0 \mathrm{~Hz}, 1 \mathrm{H}), 7.49-7.44(\mathrm{~m}, 1 \mathrm{H}), 6.81-6.74$ (major, d, J = $15.9 \mathrm{~Hz}, 1 \mathrm{H}), 6.25-6.21$ (minor, d, J = 12.5 Hz, $1 \mathrm{H}) .{ }^{13} \mathbf{C} \mathbf{N M R}\left(\mathrm{CD}_{3} \mathrm{OD}, 100 \mathrm{MHz}\right) \delta 169.9$, $150.8,149.8,138.4,136.3,132.8,125.5,124.4$. HRMS (ESI-TOF) $\mathrm{m} / \mathrm{z}:[\mathrm{M}+\mathrm{H}]^{+}$Calcd for $\mathrm{C}_{8} \mathrm{H}_{9} \mathrm{~N}_{2} \mathrm{O}^{+}$ 149.0709; Found 149.0714.

\section{(E)-3-cyclohexylacrylamide (6i)}<smiles>NC(=O)/C=C/C1CCCCC1</smiles>

Prepared according to Procedure 5.2. White solid (30.8 mg, $0.201 \mathrm{mmol}, 35 \%$, $84: 16 E: Z)$. $\mathbf{R}_{\mathbf{f}}(3: 2$ dichloromethane:ethyl acetate $)=0.29$. Purified using a $20-40 \%$ gradient of ethyl acetate in dichloromethane. ${ }^{1} \mathbf{H}$ NMR $\left(\mathrm{CD}_{3} \mathrm{OD}, 400 \mathrm{MHz}\right) \delta 6.78$ -6.71 (major, dd, $\mathrm{J}=15.6,6.9 \mathrm{~Hz}, 1 \mathrm{H}$ ), $5.93-5.87$ (major, dd, $\mathrm{J}=15.6,1.4 \mathrm{~Hz}$, $1 \mathrm{H}$ ), $5.86-5.80$ (minor, dd, $\mathrm{J}=11.6,9.6 \mathrm{~Hz}, 1 \mathrm{H}), 5.75-5.71$ (minor, dd, $\mathrm{J}=11.6$, $0.8 \mathrm{~Hz}, 1 \mathrm{H}), 2.20-2.08(\mathrm{~m}, 1 \mathrm{H}), 1.82-1.64(\mathrm{~m}, 8 \mathrm{H}), 1.41-1.10(\mathrm{~m}, 8 \mathrm{H}) .{ }^{13} \mathbf{C}$ NMR $\left(\mathrm{CD}_{3} \mathrm{OD}, 100 \mathrm{MHz}\right)$ $\delta 171.4,155.4$ (minor), 151.8, 121.9, 120.3 (minor), 41.0, 38.2 (minor), 33.7, 33.1, 27.1, 27.1 (minor), 26.9, 26.7 (minor). HRMS (ESI-TOF) m/z: $[\mathrm{M}+\mathrm{H}]^{+}$Calcd for $\mathrm{C}_{9} \mathrm{H}_{16} \mathrm{NO}^{+}$154.1226; Found 154.1219. 


\title{
7. Synthesis and Characterization of FK866 Analog: Intermediates and Final $E$ - Cinnamamide
}

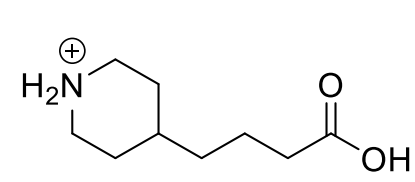

\author{
1) $\mathrm{SOCl}_{2}, \mathrm{MeOH},-10{ }^{\circ} \mathrm{C}, 16 \mathrm{~h}$ \\ 2) $\mathrm{BzCl}, \mathrm{TEA}, \mathrm{DCM},-10^{\circ} \mathrm{C}, 16 \mathrm{~h}$ \\ 3) $\mathrm{NaBH}_{4}, \mathrm{MeOH}, \mathrm{DME}, 80^{\circ} \mathrm{C}, 4 \mathrm{~h}$ \\ 4) DPPA, $\mathrm{NaN}_{3}, \mathrm{DMF}, 90^{\circ} \mathrm{C}, 30 \mathrm{~min}$
}

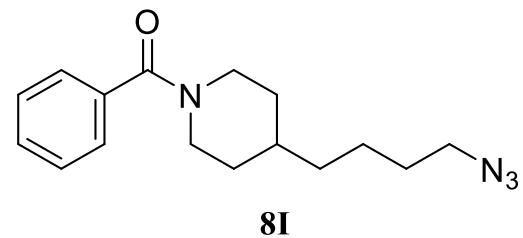

Esterification: The following procedure was adapted from a similar protocol. ${ }^{3}$ Thionyl chloride $(3.79 \mathrm{~g}$, $31.8 \mathrm{mmol}$ ) was added dropwise over 1 minute to $10 \mathrm{~mL}$ of $\mathrm{MeOH}$ at $-10{ }^{\circ} \mathrm{C}$ (brine/ice bath). To this solution was added 4-piperidine butyric acid hydrochloride $(1.99 \mathrm{~g}, 9.58 \mathrm{mmol})$ at $-10^{\circ} \mathrm{C}$. The mixture stirred at room temperature for 20 hours. The obtained clear solution was concentrated in vacuo. The remaining white solid was suspended in ether and filtered. The crude residue was carried forward to benzoylation $\left(\mathbf{R}_{\mathbf{f}}(95: 5\right.$ dichloromethane:methanol $\left.)=0.21\right)$ without further purification.

Benzoylation: To a suspension of crude residue from Esterification in $10 \mathrm{~mL}$ of dichloromethane was added benzoyl chloride $(1.48 \mathrm{~g}, 10.5 \mathrm{mmol})$ which was previously cooled to $0{ }^{\circ} \mathrm{C}$ with a brine/ice bath. To this mixture was added triethylamine $(2.7 \mathrm{~mL}, 19.2 \mathrm{mmol})$ in a dropwise fashion. The mixture stirred at room temperature overnight. The organic layer was washed with water and sat. $\mathrm{NaHCO}_{3}$ solution and dried over anhydrous sodium sulfate. The solution was concentrated in vacuo yielding a red oil as a crude product $\left(\mathbf{R}_{\mathbf{f}}(3: 2\right.$ dichloromethane:ethyl acetate $\left.)=0.68\right)$, which was carried forward without further purification.

Ester Reduction: A suspension of crude ester from Benzoylation and $\mathrm{NaBH}_{4}(1.81 \mathrm{~g}, 47.9 \mathrm{mmol})$ in 1,2dimethoxyethane $(32 \mathrm{~mL})$ was heated to $50^{\circ} \mathrm{C}$. Methanol $(7.0 \mathrm{~mL})$ was added dropwise. The reaction was then heated to $80{ }^{\circ} \mathrm{C}$ and stirred for 4 hours. Upon completion, water was carefully added to the reaction mixture. The reaction was extracted with ethyl acetate $(30 \mathrm{~mL}, 3 \mathrm{x})$. The combined organic layers were washed with brine and dried over anhydrous sodium sulfate. The organic layer was concentrated in vacuo $\left(\mathbf{R}_{\mathbf{f}}(3: 2\right.$ dichloromethane:ethyl acetate $\left.)=0.21\right)$ and carried forward without further purification.

Alcohol to Azide: The following procedure was adapted from a similar protocol. ${ }^{4}$ The crude reaction mixture from Ester Reduction was added to a 6 dram vial and purged with $\mathrm{N}_{2}$ via Schlenk technique. The reaction was diluted with dry DMF $(32 \mathrm{~mL})$, followed by addition of DPPA $(4.32 \mathrm{~mL}, 20.1 \mathrm{mmol})$ and sodium azide $(1.31 \mathrm{~g}, 20.1 \mathrm{mmol})$. To the resulting solution was added DBU (3.0 mL, $20.1 \mathrm{mmol})$ in a dropwise fashion. The reaction was heated to $90{ }^{\circ} \mathrm{C}$ for 30 minutes. The reaction was diluted with water (20 $\mathrm{mL})$ and diethyl ether $(30 \mathrm{~mL})$. The organic layer was extracted and washed with water $(20 \mathrm{~mL}, 2 \mathrm{x})$. The combined organic extracts were washed with brine, dried over anhydrous sodium sulfate, and concentrated in vасио. The residue was purified by silica gel column chromatography (eluted with a $10-30 \%$ gradient of ethyl acetate in hexanes solvent system) yielding the $\mathbf{8 I}$ as a yellow oil.

\section{(4-(4-azidobutyl)piperidin-1-yl)(phenyl)methanone (8I)}

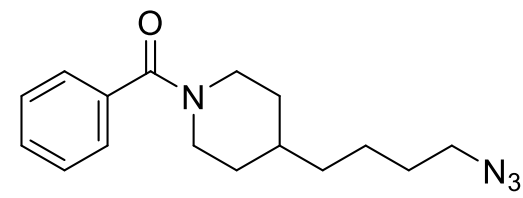

Yellow oil (1.34 g, $4.68 \mathrm{mmol}, 49 \%)$. $\mathbf{R}_{\mathbf{f}}$ (3:2 dichloromethane:ethyl acetate $)=0.76 .{ }^{1} \mathbf{H}$ NMR $\left(\mathrm{CDCl}_{3}, 400 \mathrm{MHz}\right) \delta 7.41-7.36(\mathrm{~m}, 5 \mathrm{H})$, $4.77-4.64(\mathrm{~m}, 1 \mathrm{H}), 3.81-3.66(\mathrm{~m}, 1 \mathrm{H}), 3.30-3.24(\mathrm{t}, \mathrm{J}=7.2 \mathrm{~Hz}$, $2 \mathrm{H}), 3.04-2.66(\mathrm{~m}, 2 \mathrm{H}), 1.88-1.72(\mathrm{~m}, 1 \mathrm{H}), 1.72-1.46(\mathrm{~m}, 5 \mathrm{H})$, $1.46-1.34(\mathrm{~m}, 2 \mathrm{H}), 1.34-1.04(\mathrm{~m}, 5 \mathrm{H}) .{ }^{13} \mathbf{C ~ N M R}\left(\mathrm{CDCl}_{3}, 100 \mathrm{MHz}\right)$

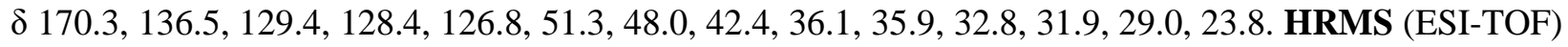


m/z: $[\mathrm{M}+\mathrm{H}]^{+}$Calcd for $\mathrm{C}_{16} \mathrm{H}_{23} \mathrm{~N}_{4} \mathrm{O}^{+}$287.1866; Found 287.1851. The data are in accordance with those reported in the literature. ${ }^{[4]}$

Azide to Amine:<smiles>N#CCCCC1CCN(C(=O)c2ccccc2)CC1</smiles>

$8 I$

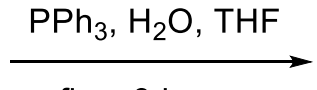

reflux, $3 \mathrm{~h}$<smiles>NCCCCC1CCN(C(=O)c2ccccc2)CC1</smiles>

8 II

The following procedure was adapted from a similar protocol. ${ }^{4} \mathrm{~A} 100 \mathrm{~mL}$ round bottom flask was charged with azide $8 \mathrm{I}$ (1.34 g, $4.68 \mathrm{mmol})$ followed by dilution with THF $(47 \mathrm{~mL})$. To the stirred solution was added triphenylphosphine $(1.23 \mathrm{~g}, 4.68 \mathrm{mmol})$ and water $(0.51 \mathrm{~mL}, 28.1 \mathrm{mmol})$. The reaction was heated to reflux for 3 hours. The reaction mixture was concentrated in vacuo and purified by silica gel column chromatography (eluted using a 10\% (98:2 methanol: $\left.\mathrm{NH}_{4} \mathrm{OH}\right)$ in ethyl acetate isocratic solvent system).

\section{(4-(4-aminobutyl)piperidin-1-yl)(phenyl)methanone (8II)}

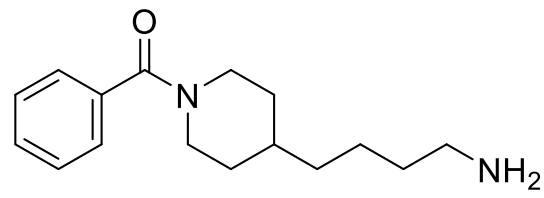

Light orange oil (994 mg, $3.82 \mathrm{mmol}, 81.6 \%)$. $\mathbf{R}_{\mathbf{f}}$ (9:1 ethyl acetate: $\left(98: 2\right.$ methanol: $\left.\left.\mathrm{NH}_{4} \mathrm{OH}\right)\right)=0.05$. ${ }^{1} \mathbf{H}$ NMR $\left(\mathrm{CD}_{3} \mathrm{OD}, 400\right.$ MHz) $\delta 7.49-7.42(\mathrm{~m}, 3 \mathrm{H}), 7.41-7.35(\mathrm{~m}, 2 \mathrm{H}), 4.66-4.55(\mathrm{~m}$, $1 \mathrm{H}), 3.75-3.64(\mathrm{~m}, 1 \mathrm{H}), 3.28-3.22(\mathrm{t}, \mathrm{J}=6.7 \mathrm{~Hz}, 1 \mathrm{H}), 3.13-3.02$ $(\mathrm{t}, \mathrm{J}=12.4 \mathrm{~Hz}, 1 \mathrm{H}), 2.90-2.77(\mathrm{t}, \mathrm{J}=12.2 \mathrm{~Hz}, 1 \mathrm{H}), 2.72-2.67(\mathrm{t}, \mathrm{J}$ $=6.8 \mathrm{~Hz}, 1 \mathrm{H}), 1.90-1.77(\mathrm{~m}, 2 \mathrm{H}), 1.74-1.46(\mathrm{~m}, 5 \mathrm{H}), 1.45-1.27(\mathrm{~m}, 5 \mathrm{H}), 1.27-1.01(\mathrm{~m}, 3 \mathrm{H}) .{ }^{13} \mathbf{C}$ NMR $\left(\mathrm{CD}_{3} \mathrm{OD}, 100 \mathrm{MHz}\right) \delta 170.9,135.9,129.5,128.3,126.3,54.6,51.9,43.7,42.2,37.3,37.3,37.2,37.2$, 33.9, 33.1, 31.6, 25.6, 24.4. HRMS (ESI-TOF) m/z: $[\mathrm{M}+\mathrm{H}]^{+}$Calcd for $\mathrm{C}_{16} \mathrm{H}_{25} \mathrm{~N}_{2} \mathrm{O}^{+}$261.1961; Found 261.1958. The data are in accordance with those reported in the literature. ${ }^{[4]}$

\section{Propiolamide synthesis:}<smiles>NCCCCC1CCN(C(=O)c2ccccc2)CC1</smiles>

8II 3-phenylpropiolic acid DCC, DMAP, DCM

$0{ }^{\circ} \mathrm{C}, 16 \mathrm{~h}$

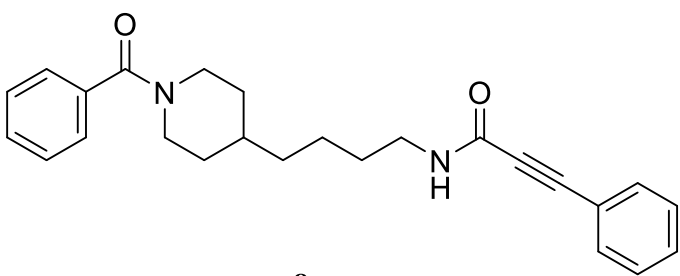

8

$N$-(4-(1-benzoylpiperidin-4-yl)butyl)-3-phenylpropiolamide (8)

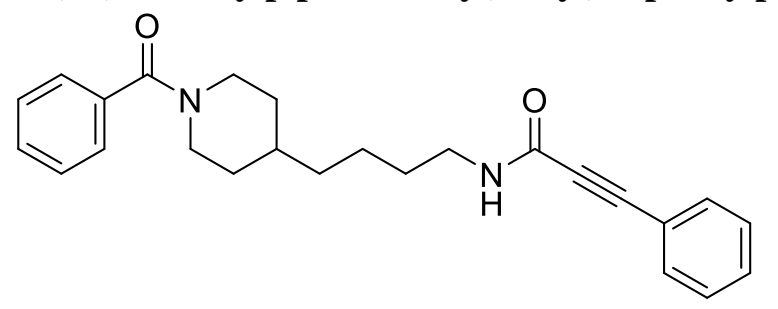

Prepared according to Procedure 3.3. Light orange oil (209 mg, 0.539 mmol, 67\%). $\mathbf{R}_{\mathbf{f}} \quad(3: 2$ dichloromethane:ethyl acetate) $=0.45 .{ }^{1} \mathbf{H} \mathbf{~ N M R}$ $\left(\mathrm{CD}_{3} \mathrm{OD}, 400 \mathrm{MHz}\right) \delta 7.61-7.54(\mathrm{~m}, 2 \mathrm{H}), 7.50-7.33$ $(\mathrm{m}, 8 \mathrm{H}), 4.66-4.56(\mathrm{~m}, 1 \mathrm{H}), 3.74-3.65(\mathrm{~m}, 1 \mathrm{H}), 3.29$ $-3.24(\mathrm{t}, \mathrm{J}=6.6 \mathrm{~Hz}, 2 \mathrm{H}), 3.14-3.01(\mathrm{t}, \mathrm{J}=12.7 \mathrm{~Hz}$, $1 \mathrm{H}), 2.90-2.77(\mathrm{t}, \mathrm{J}=12.5 \mathrm{~Hz}, 1 \mathrm{H}), 1.92-1.79(\mathrm{~m}$,

$1 \mathrm{H}), 1.75-1.65(\mathrm{~m}, 1 \mathrm{H}), 1.65-1.51(\mathrm{~m}, 3 \mathrm{H}), 1.48-1.28(\mathrm{~m}, 5 \mathrm{H}), 1.28-1.04(\mathrm{~m}, 3 \mathrm{H}) .{ }^{13} \mathrm{C} \mathrm{NMR}\left(\mathrm{CD}_{3} \mathrm{OD}\right.$, $126 \mathrm{MHz}) \delta 172.4,155.7,137.4, * 133.5,133.5, * 131.7,131.3,130.9,130.0,129.8,129.7,127.7,121.6$, 85.7, 83.8, 57.5, 43.7, 40.7, 37.1, 37.0, 33.9, 33.1, 30.3, 25.0. HRMS (ESI-TOF) m/z: $[\mathrm{M}+\mathrm{H}]^{+}$Calcd for $\mathrm{C}_{25} \mathrm{H}_{29} \mathrm{~N}_{2} \mathrm{O}_{2}{ }^{+}$389.2224; Found 389.2221. 
(E)-N-(4-(1-benzoylpiperidin-4-yl)butyl)cinnamamide (9)

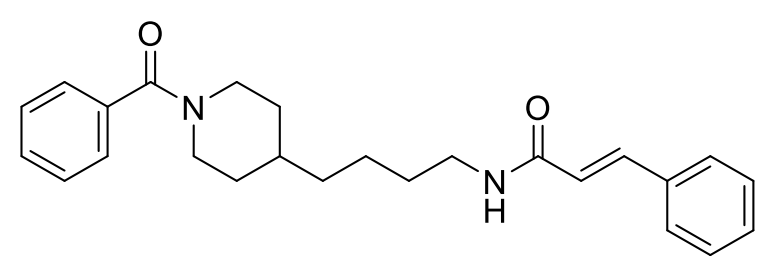

Prepared according to Procedure 5.1. Light orange oil (159 mg, 0.409 mmol, 76\%, >99:1 E:Z). $\mathbf{R}_{\mathbf{f}}(3: 2$ dichloromethane:ethyl acetate) $=0.42 .{ }^{1} \mathbf{H}$ NMR $\left(\mathrm{CDCl}_{3}, 400 \mathrm{MHz}\right) \delta 7.64-7.59(\mathrm{~d}, \mathrm{~J}=15.8 \mathrm{~Hz}, 1 \mathrm{H})$, $7.51-7.46(\mathrm{~m}, 2 \mathrm{H}), 7.41-7.31(\mathrm{~m}, 8 \mathrm{H}), 6.40-6.35$ $(\mathrm{d}, \mathrm{J}=15.3 \mathrm{~Hz}, 1 \mathrm{H}), 5.73-5.66(\mathrm{t}, \mathrm{J}=5.3 \mathrm{~Hz}, 1 \mathrm{H}), 4.76$ $-4.63(\mathrm{~m}, 1 \mathrm{H}), 3.79-3.65(\mathrm{~m}, 1 \mathrm{H}), 3.42-3.34(\mathrm{q}, \mathrm{J}=7.1 \mathrm{~Hz}, 2 \mathrm{H}), 3.03-2.65(\mathrm{~m}, 2 \mathrm{H}), 1.87-1.70(\mathrm{~m}$, 1H), $1.70-1.44(\mathrm{~m}, 6 \mathrm{H}), 1.44-1.25(\mathrm{~m}, 5 \mathrm{H}), 1.25-1.01(\mathrm{~m}, 3 \mathrm{H}) .{ }^{13} \mathbf{C} \mathbf{N M R}\left(\mathrm{CDCl}_{3}, 126 \mathrm{MHz}\right) \delta .170 .4$, 166.0, 141.1, 136.6, 135.0, 129.8, 129.6, 129.0, 128.6, 127.9, 127.0, 120.8, 48.2, 42.6, 39.8, 36.2, 36.2, 33.0, 32.1, 30.1, 25.0, 24.1. HRMS (ESI-TOF) m/z: $[\mathrm{M}+\mathrm{H}]^{+}$Calcd for $\mathrm{C}_{25} \mathrm{H}_{31} \mathrm{~N}_{2} \mathrm{O}_{2}{ }^{+}$391.2380; Found 391.2370 . 


\section{Deuterium labeling study:}

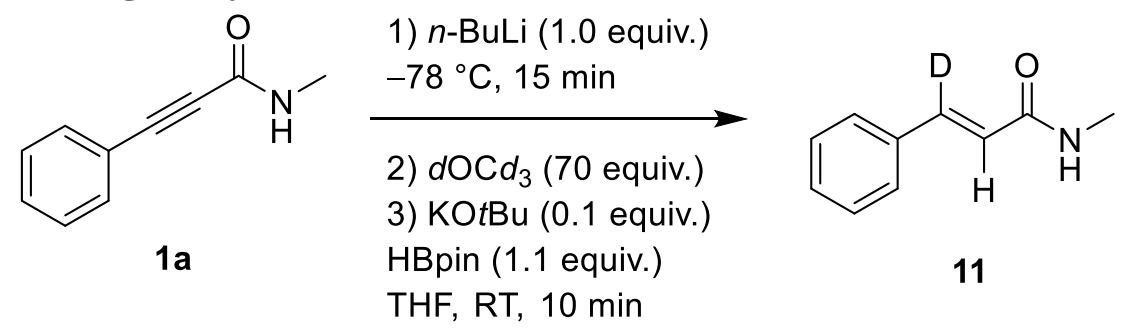

A flame-dried $5 \mathrm{~mL}$ round bottom flask was charged with dry propiolamide ( $93 \mathrm{mg}, 0.58 \mathrm{mmol})$ and purged with $\mathrm{N}_{2}$ via Schlenk technique. The solid alkynamide was then heated to $100{ }^{\circ} \mathrm{C}$ while purging the round bottom flask with argon for 1 hour. The reaction was diluted with THF $(2.7 \mathrm{~mL}, 0.2 \mathrm{M})$ and then cooled to $0{ }^{\circ} \mathrm{C}$ with a ice/brine bath. After cooling the reaction for 15 minutes, $\mathrm{NaH}(17 \mathrm{mg}, 0.64 \mathrm{mmol}, 90 \mathrm{wt} \%)$ was added dropwise. The reaction was stirred for 30 minutes at $0{ }^{\circ} \mathrm{C} . d \mathrm{OC} d_{3}(1.0 \mathrm{~mL}, 26.9 \mathrm{mmol})$ was added dropwise. The reaction was stirred at $0{ }^{\circ} \mathrm{C}$ for 30 minutes before warming to room temperature by removing the ice/brine bath. The reaction was concentrated in vacuo and dried on high vacuum overnight to remove residual $d \mathrm{OC} d_{3}$. The ${ }^{1} \mathrm{H}$ NMR of the deuterated amide revealed $>99 \% d$-incorporation of the amide. The resulting white solid was diluted with THF $(1.2 \mathrm{~mL}, 0.5 \mathrm{M})$. Freshly dried $\mathrm{KO} t \mathrm{Bu}(6.6 \mathrm{mg}$, $0.058 \mathrm{mmol})$ was added to the reaction mixture. Pinacolborane $(93 \mu \mathrm{L}, 0.64 \mathrm{mmol})$ was slowly added to the reaction dropwise (1 drop ever 3-4 seconds). The reaction was stirred for 10 minutes at room temperature. The reaction mixture was concentrated in vacuo and the crude reaction subjected to ${ }^{1} \mathrm{H}$ NMR analysis. A proton signal was visible at $6.4 \mathrm{ppm}$, which represents the proton signal on the $\alpha$-carbon of $2 \mathbf{a}$, but the proton signal at $7.6 \mathrm{ppm}$, which represents the proton on the $\beta$-carbon of $\mathbf{2 a}$, was not present. The reaction was purified by silica gel column chromatography (eluted with a 20-30\% gradient of ethyl acetate in dichloromethane) yielding a white solid. The purified reaction mixture contained $c a$. 1:1 2a:11.

\section{(E)- $N$-methylcinnamamide-3- $d(11)$}

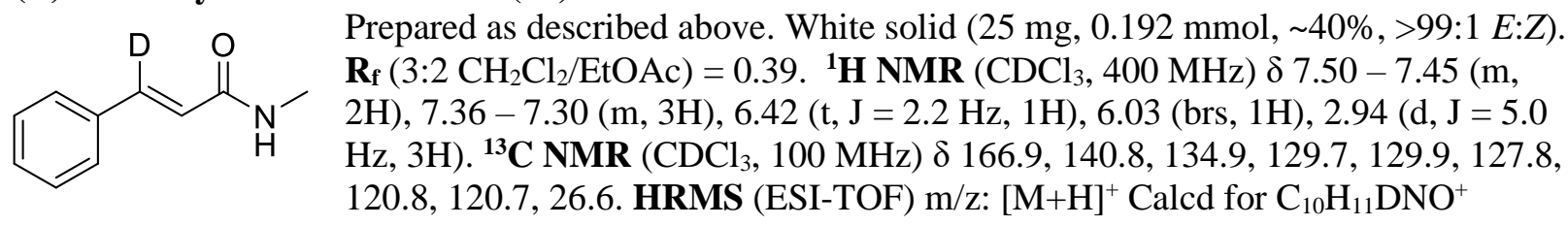

163.0976; Found 163.0975.

\section{Characterization of $E$ - $\beta$-borylacrylamide (12)}

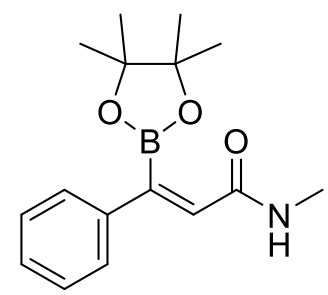

${ }^{1} \mathbf{H}$ NMR $\left(\mathrm{CDCl}_{3}, 400 \mathrm{MHz}\right) \delta$ 10.34-10.29 (m, 1H), 7.50-7.45 (m, 2H), 7.31-7.23 $(\mathrm{m}, 3 \mathrm{H}), 6.32(\mathrm{~s}, 1 \mathrm{H}), 2.62(\mathrm{~d}, \mathrm{~J}=4.8 \mathrm{~Hz}, 3 \mathrm{H}), 1.28(\mathrm{~s}, 12 \mathrm{H}) .{ }^{13} \mathbf{C ~ N M R}\left(\mathrm{CDCl}_{3}, 100\right.$ MHz) $\delta 174.3,137.8,129.0,128.3,127.9,120.5,80.8,27.1,26.4 .{ }^{11} \mathbf{B ~ N M R}\left(\mathrm{CDCl}_{3}\right.$, $128 \mathrm{MHz}$ ) $\delta$ 13.1. HRMS (ESI-TOF) $\mathrm{m} / \mathrm{z}:[\mathrm{M}+\mathrm{H}]^{+}$Calcd for $\mathrm{C}_{16} \mathrm{H}_{23} \mathrm{BNO}_{3}{ }^{+}$ 288.1766; Found 288.1779. The data are in accordance with previous literature. ${ }^{5}$ 


\section{Procedures for Control reactions with $\mathrm{BH}_{3}$ (Scheme $7 \mathrm{C}$ in the manuscript)}

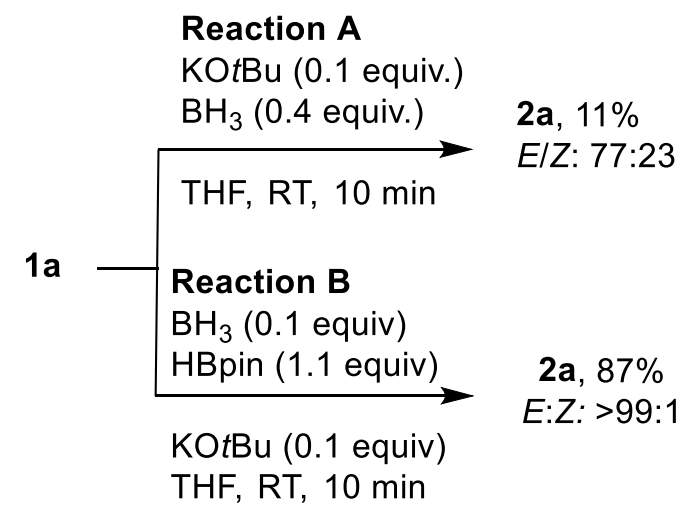

Reaction A: An oven- or flame-dried $5 \mathrm{~mL}$ round bottom flask was charged with dry propiolamide (39 $\mathrm{mg}$, $0.24 \mathrm{mmol})$ and placed under $\mathrm{N}_{2}$ via Schlenk technique. The reaction was diluted with THF $(0.2 \mathrm{~mL}, 1 \mathrm{M}$ alkynamide) and stirred at room temperature. $\mathrm{KO} t \mathrm{Bu}(0.024 \mathrm{mmol})$ was added as a solid to the round bottom flask. Borane $(0.27 \mathrm{~mL}, 0.27 \mathrm{mmol}, 1 \mathrm{M}$ in THF) was then slowly added dropwise (1 drop every 3-4 seconds). The reaction was stirred at room temperature for 10 minutes. The reaction mixture was concentrated in vacuo and the resulting crude residue was purified by silica gel column chromatography (eluted with a 30-40\% gradient ethyl acetate in dichloromethane) to afford the product as a white solid (4.2 $\mathrm{mg}, 0.26 \mathrm{mmol}, 77: 23 \mathrm{E:Z}$ ). Where applicable, the $E$-isomer is labeled as major when the $Z$-isomer is present, which is labeled as minor.

Reaction B: An oven- or flame-dried $5 \mathrm{~mL}$ round bottom flask was charged with dry propiolamide (33 mg, $0.21 \mathrm{mmol}$ ) and placed under $\mathrm{N}_{2}$ via Schlenk technique. The reaction was diluted with THF $(0.42 \mathrm{~mL}, 0.5$ $\mathrm{M}$ alkynamide $)$ and stirred at room temperature. $\mathrm{KO} t \mathrm{Bu}(0.021 \mathrm{mmol})$ was added as a solid to the round bottom flask. In a separate flame-dried 2 dram vial, borane $(0.021 \mathrm{~mL}, 0.021 \mathrm{mmol}, 1 \mathrm{M}$ in THF) was mixed with pinacolborane $(0.033 \mathrm{~mL}, 0.23 \mathrm{mmol})$ and then slowly added dropwise (1 drop every $3-4$ seconds). The reaction was stirred at room temperature for 10 minutes. The reaction mixture was concentrated in vacuo and the resulting crude residue was purified by silica gel column chromatography (eluted with a 30-40\% gradient ethyl acetate in dichloromethane) to afford the product as a white solid (29 $\mathrm{mg}, 0.18 \mathrm{mmol},>99: 1 \mathrm{E:Z}$ ). Where applicable, the $E$-isomer is labeled as major when the Z-isomer is present, which is labeled as minor. 
11. NMR Spectra of Propiolamides

${ }^{1} \mathrm{H}$ NMR $\left(\mathrm{CDCl}_{3} 400 \mathrm{MHz}\right)$ of $1 \mathrm{~d}$

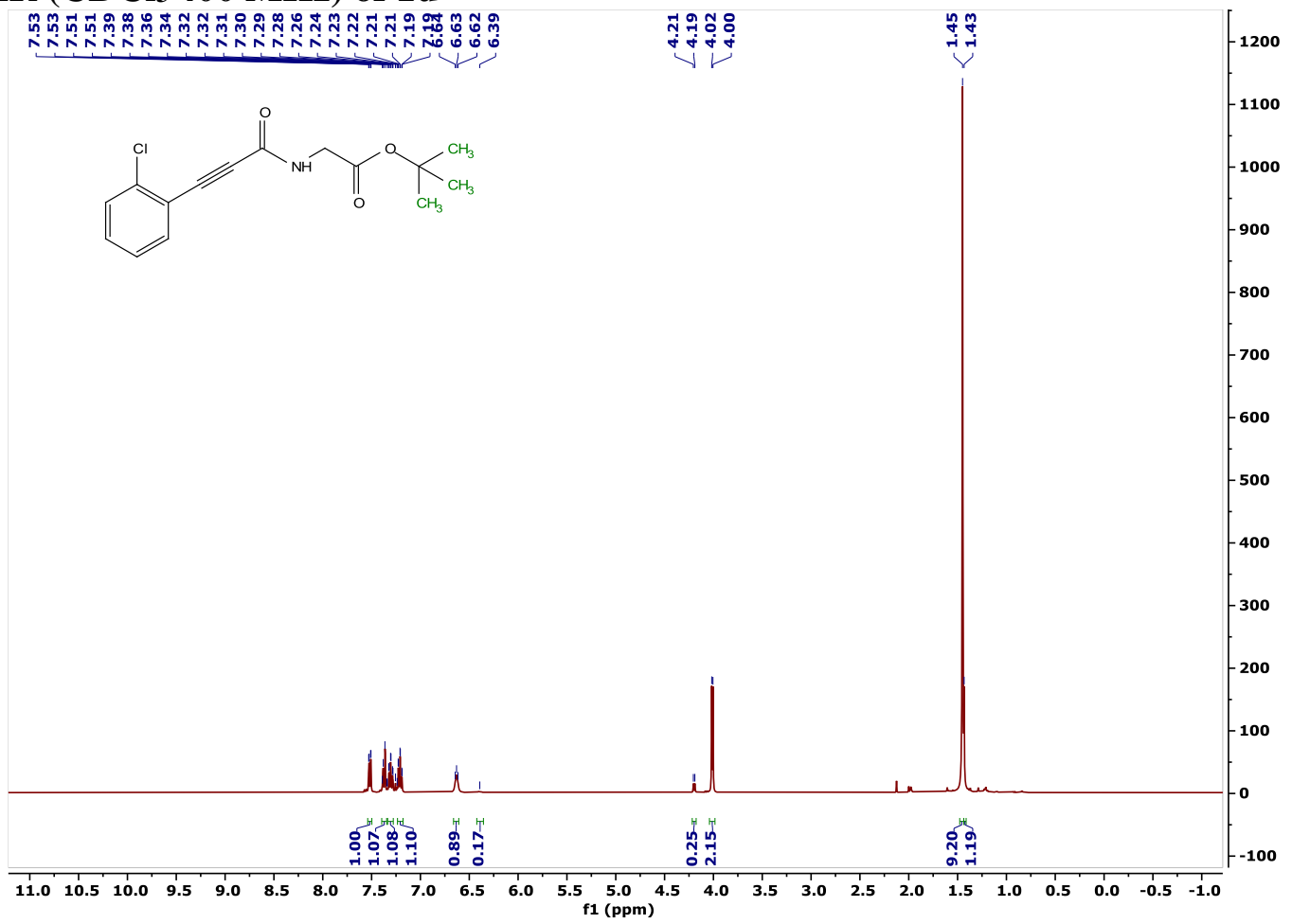

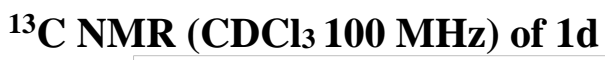

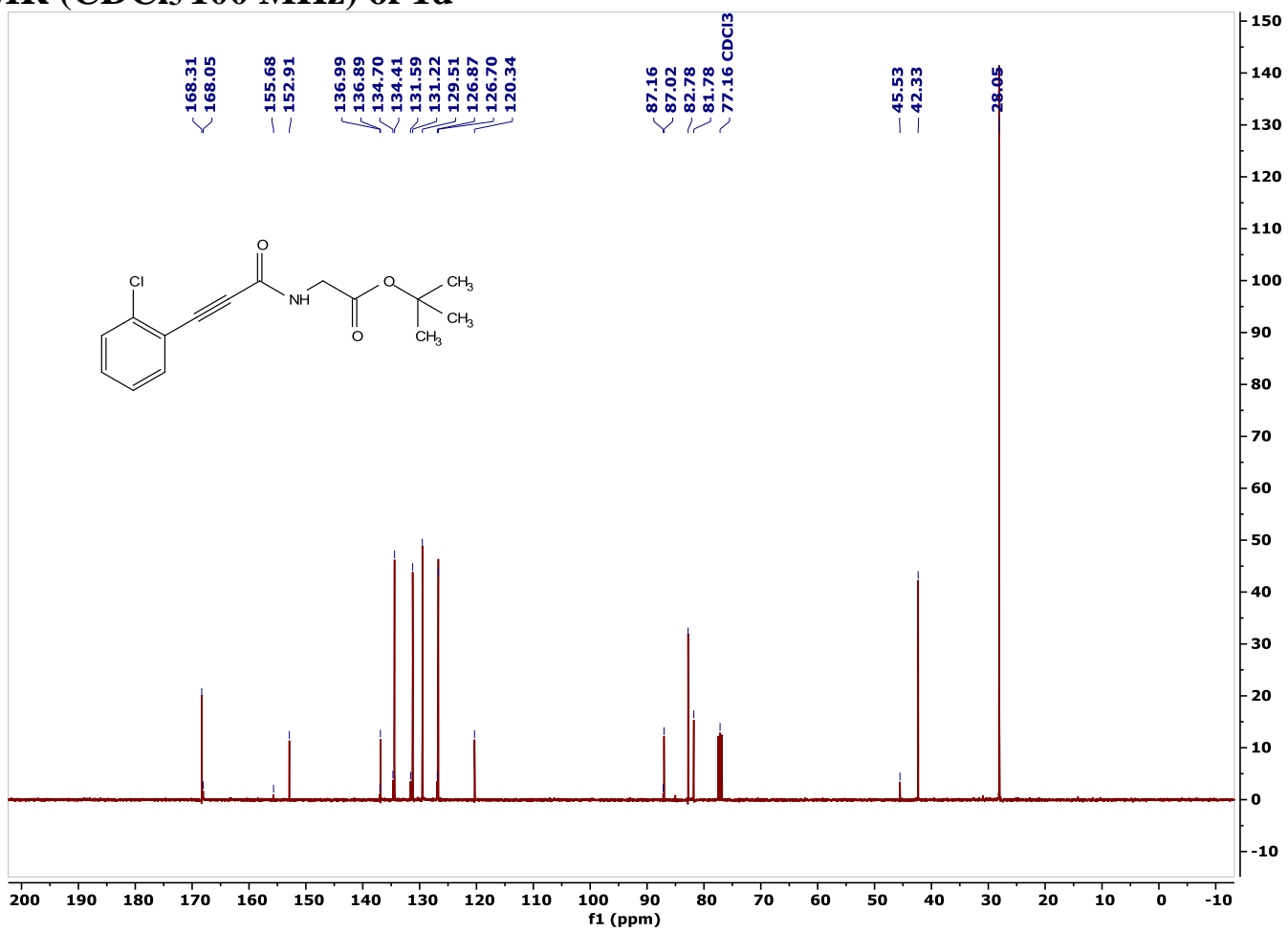


${ }^{1} \mathrm{H}$ NMR (Acetone-d $6500 \mathrm{MHz}$ ) of 11

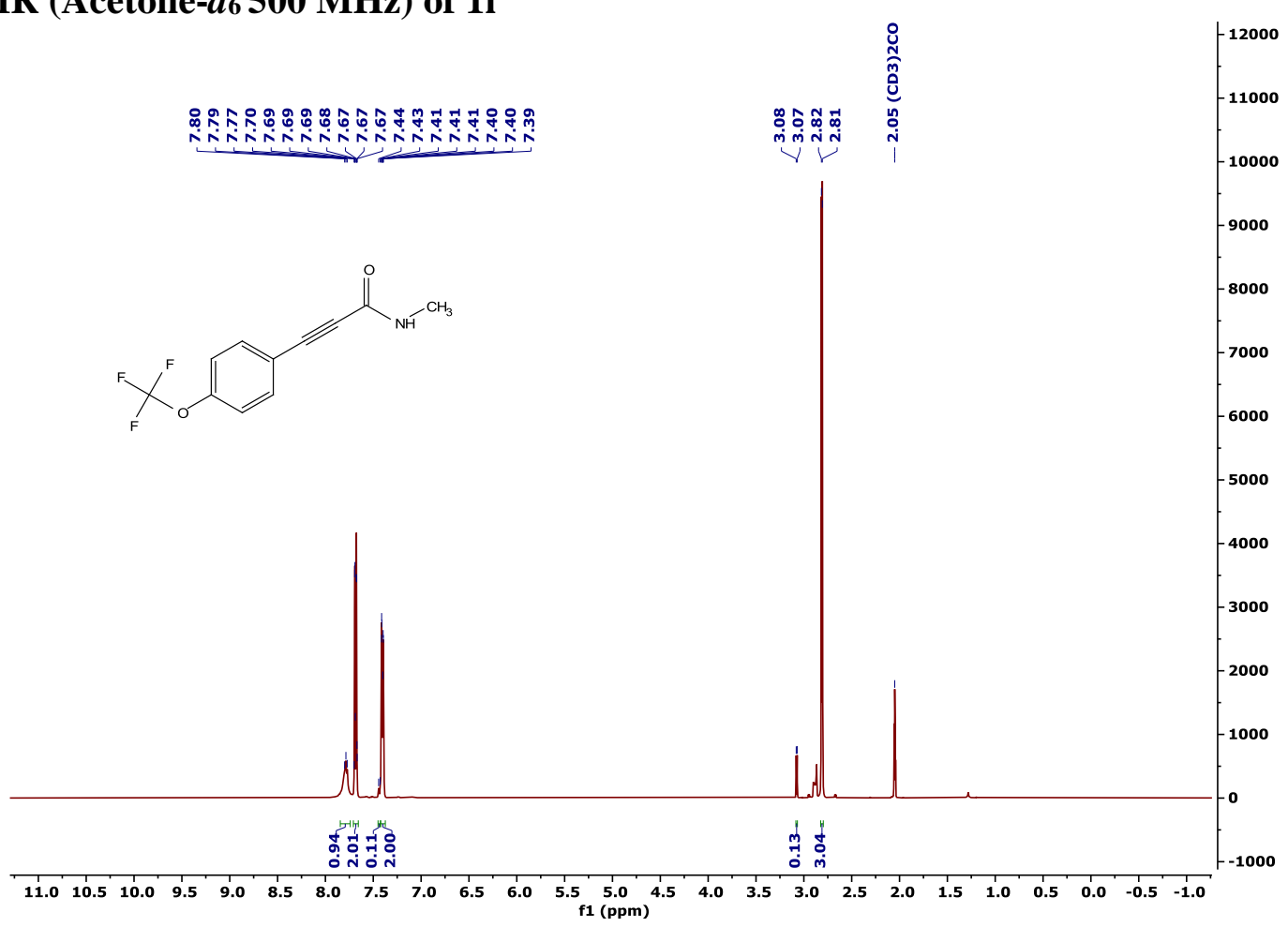

${ }^{19}$ F NMR (Acetone- $d_{6} 376 \mathrm{MHz}$ ) of 11

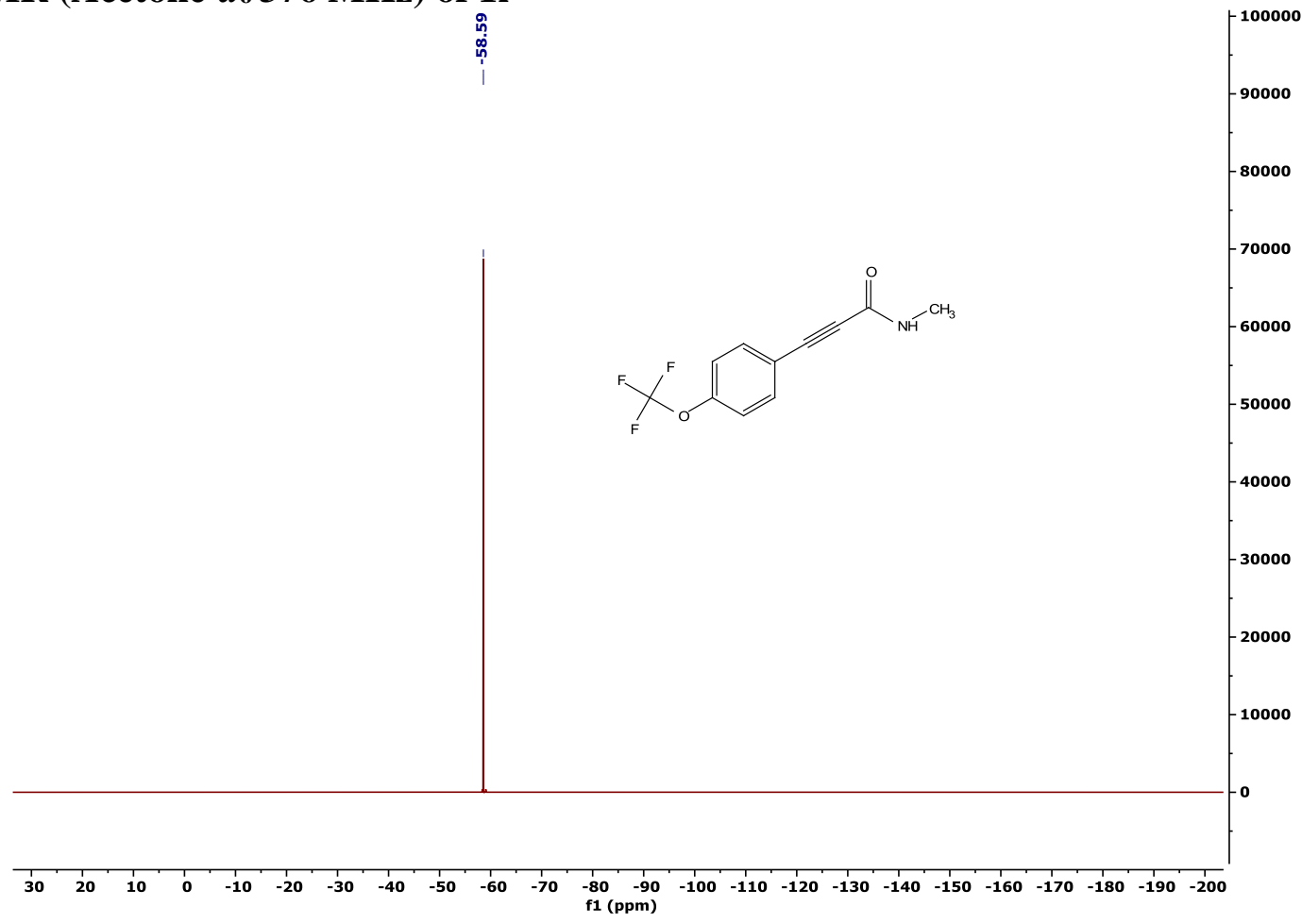


${ }^{13} \mathrm{C}$ NMR (Acetone- $d_{6} 100 \mathrm{MHz}$ ) of $1 \mathrm{I}$

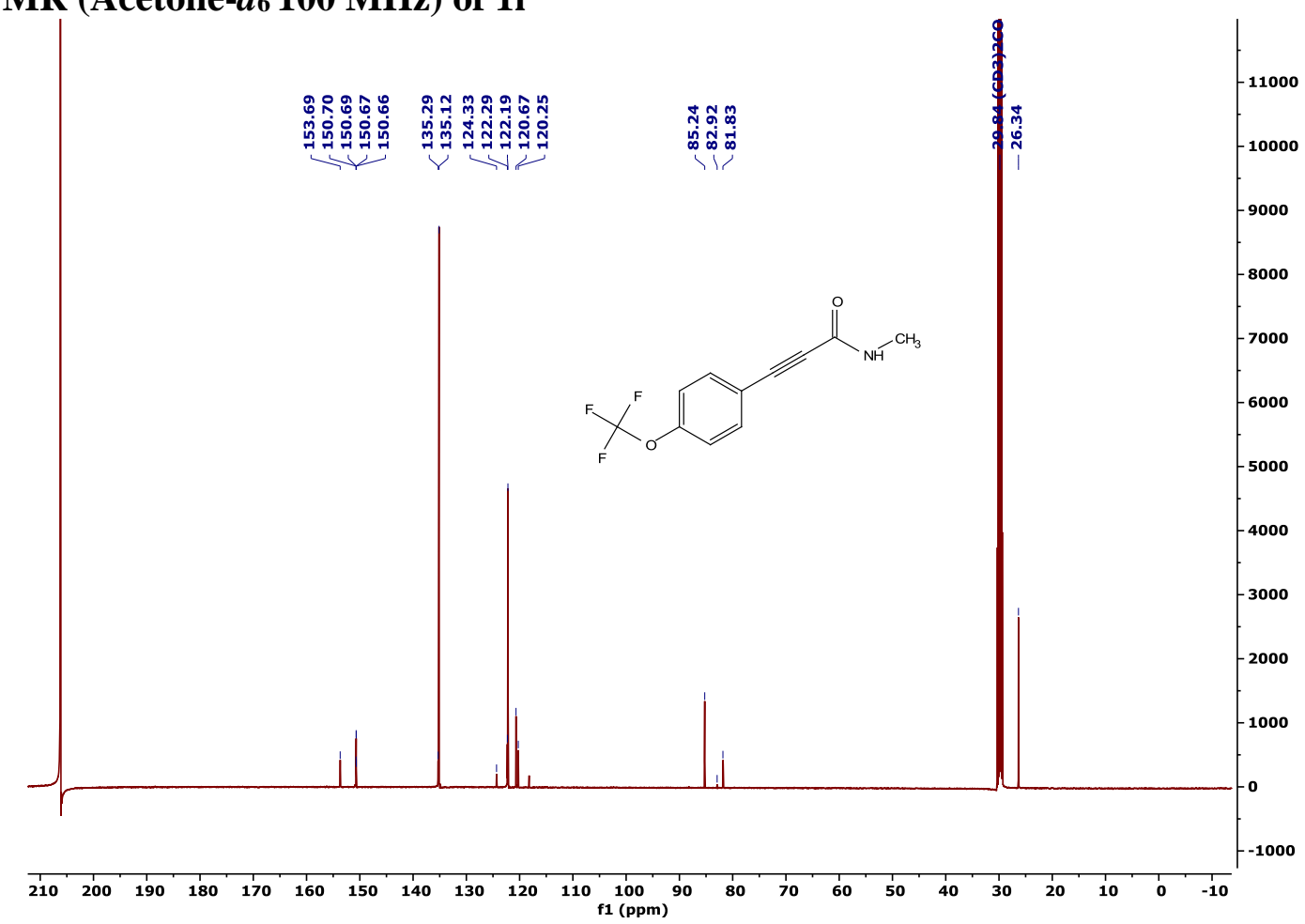

${ }^{1} \mathrm{H}$ NMR (CD ${ }_{3} \mathrm{OD} 400 \mathrm{MHz}$ ) of $1 \mathrm{p}$

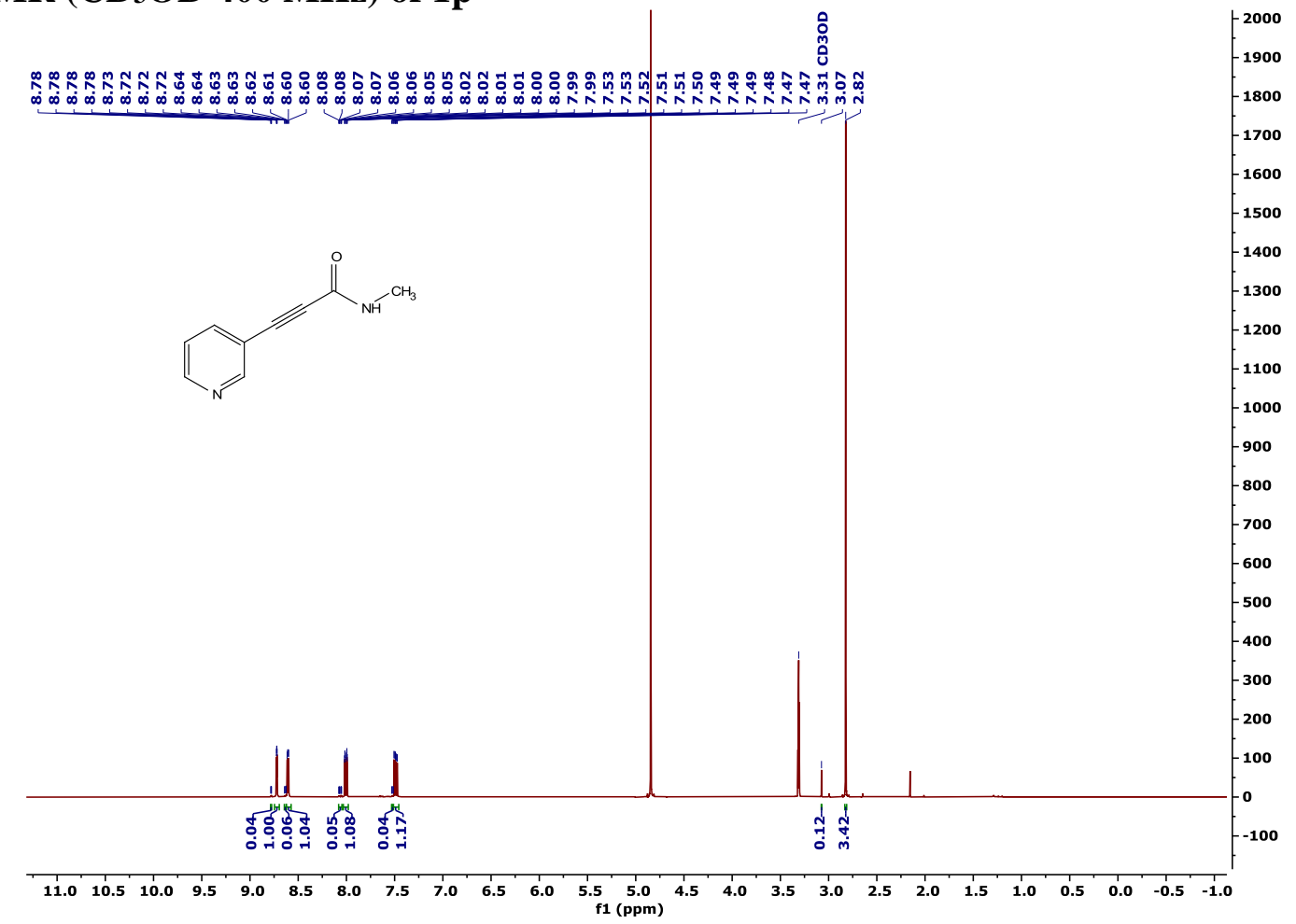


${ }^{13} \mathrm{C}$ NMR (Acetone- $d_{6} 100 \mathrm{MHz}$ ) of $1 \mathrm{p}$

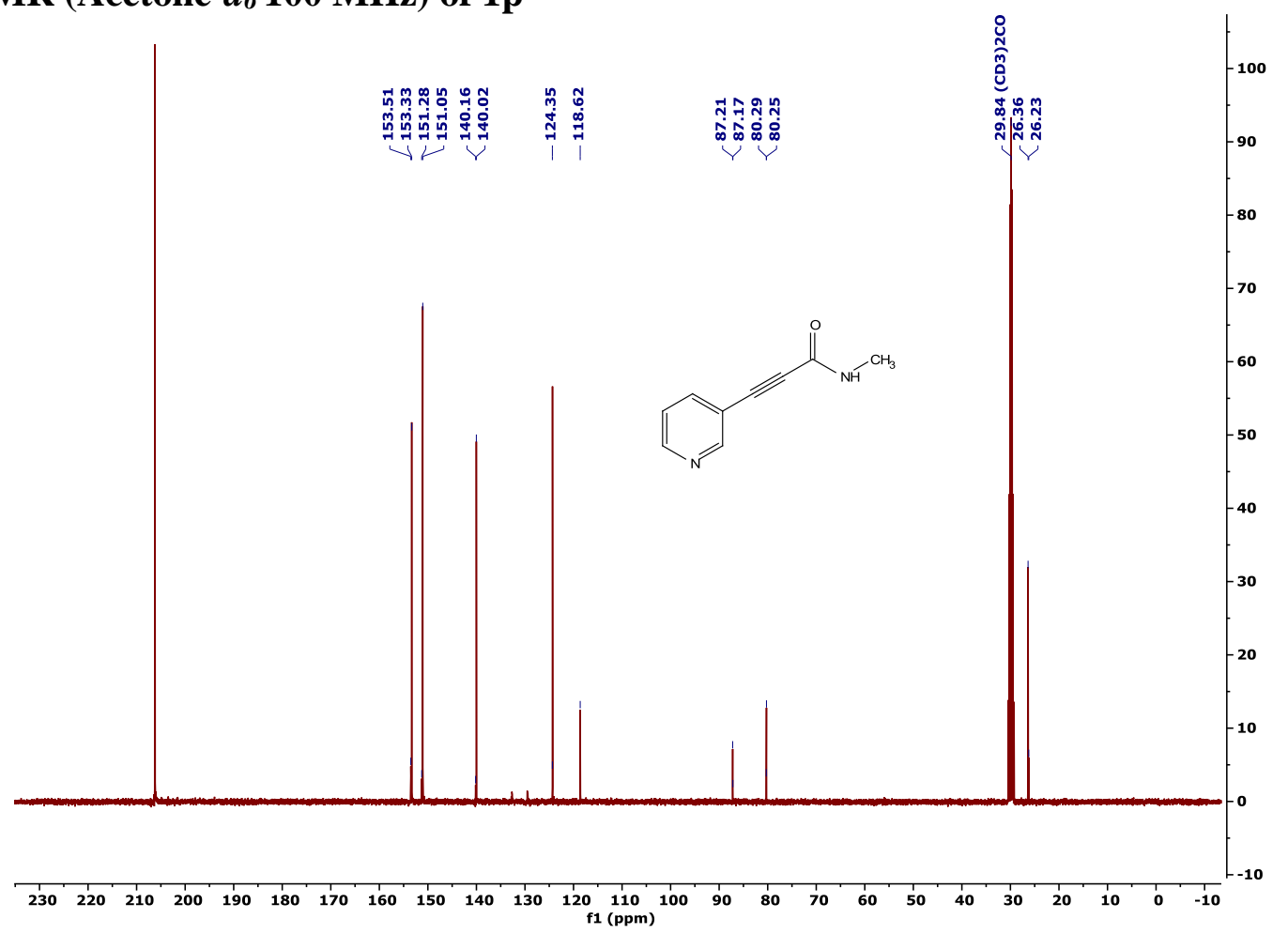

${ }^{1} \mathrm{H} \mathrm{NMR}\left(\mathrm{CDCl}_{3} 400 \mathrm{MHz}\right)$ of $3 \mathrm{c}$

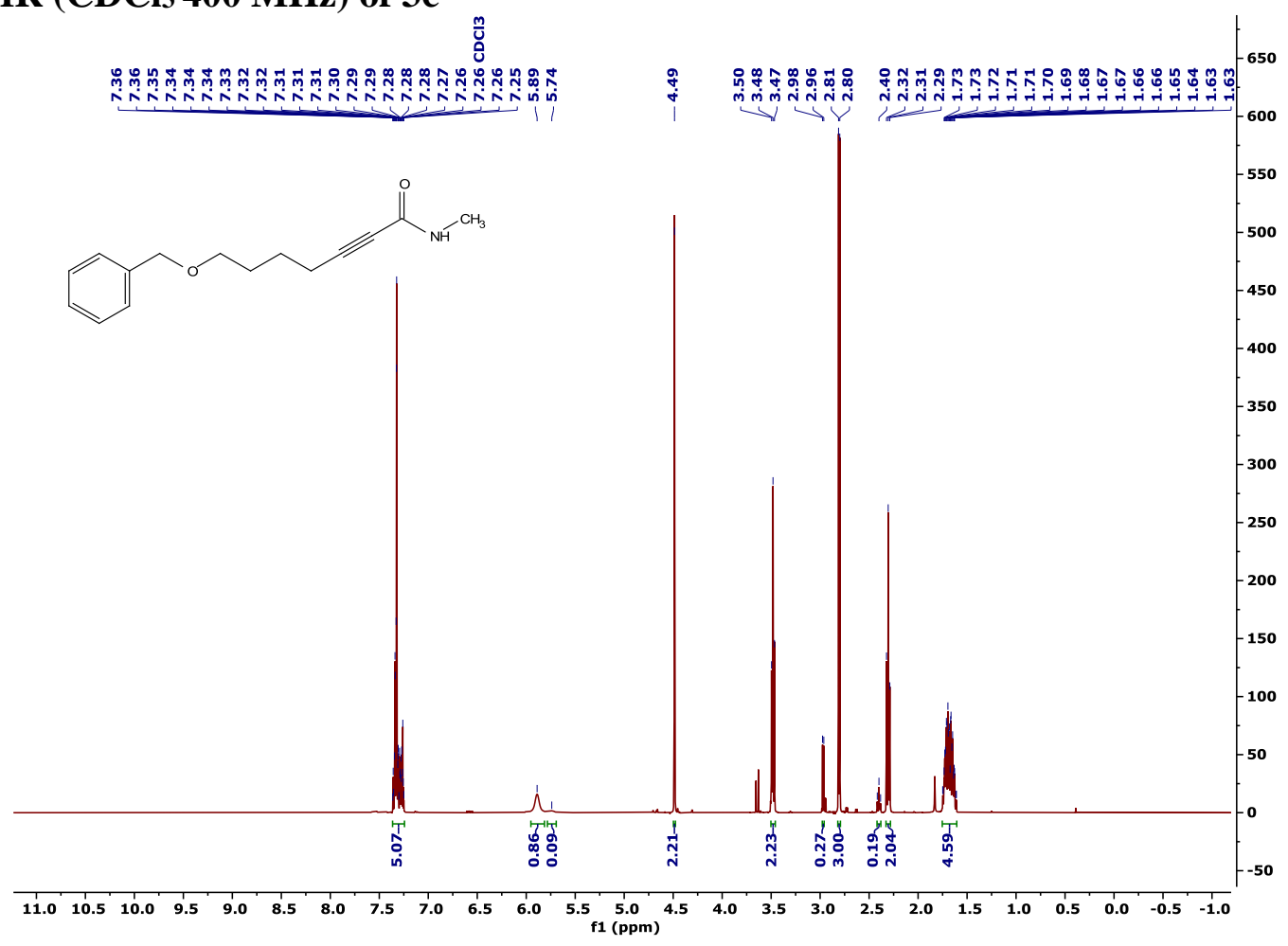


${ }^{13} \mathrm{C}$ NMR $\left(\mathrm{CDCl}_{3} 100 \mathrm{MHz}\right)$ of $3 \mathrm{c}$

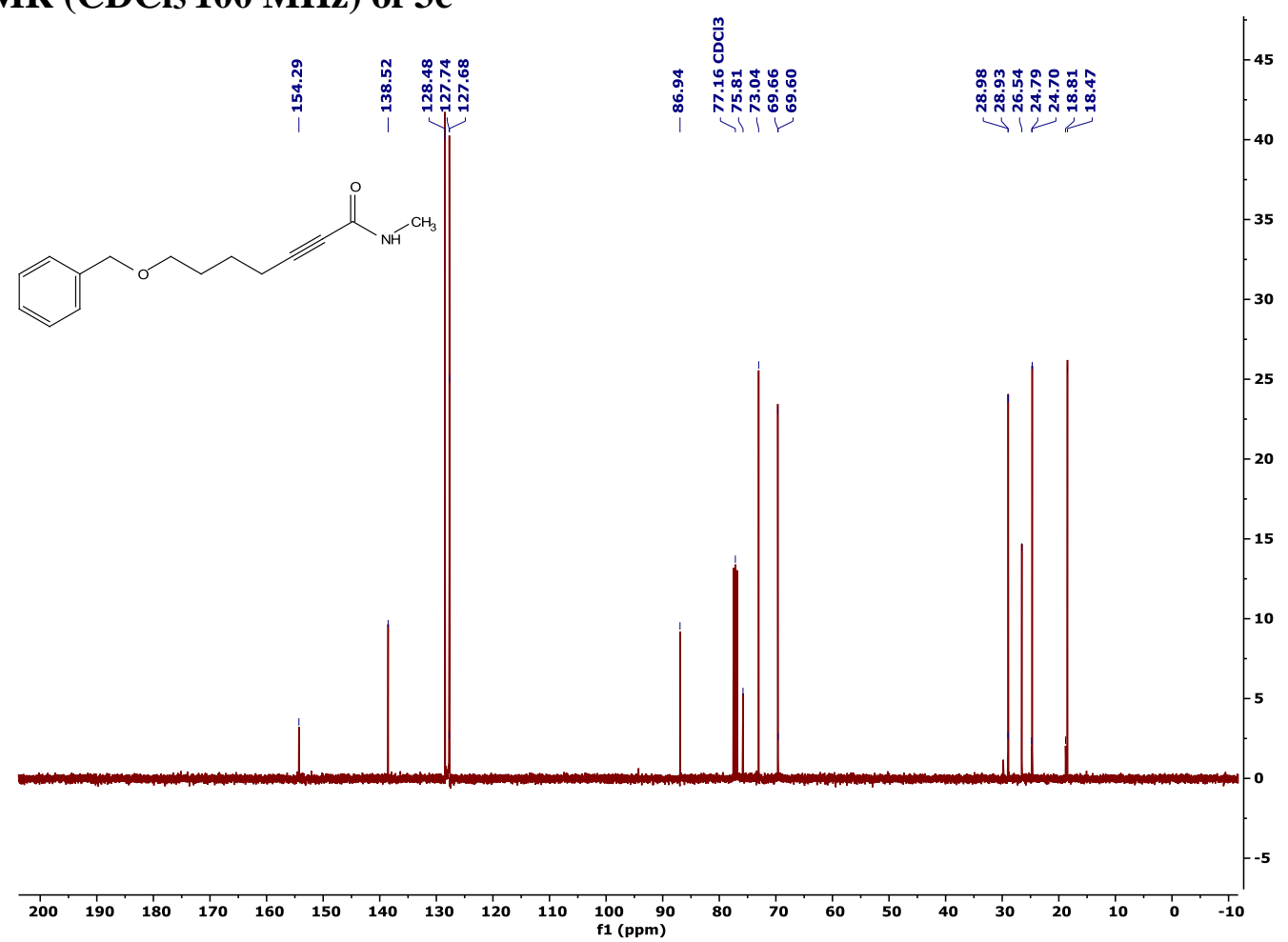

${ }^{1} \mathrm{H} \mathrm{NMR}\left(\mathrm{CDCl}_{3} 400 \mathrm{MHz}\right)$ of $3 \mathrm{f}$

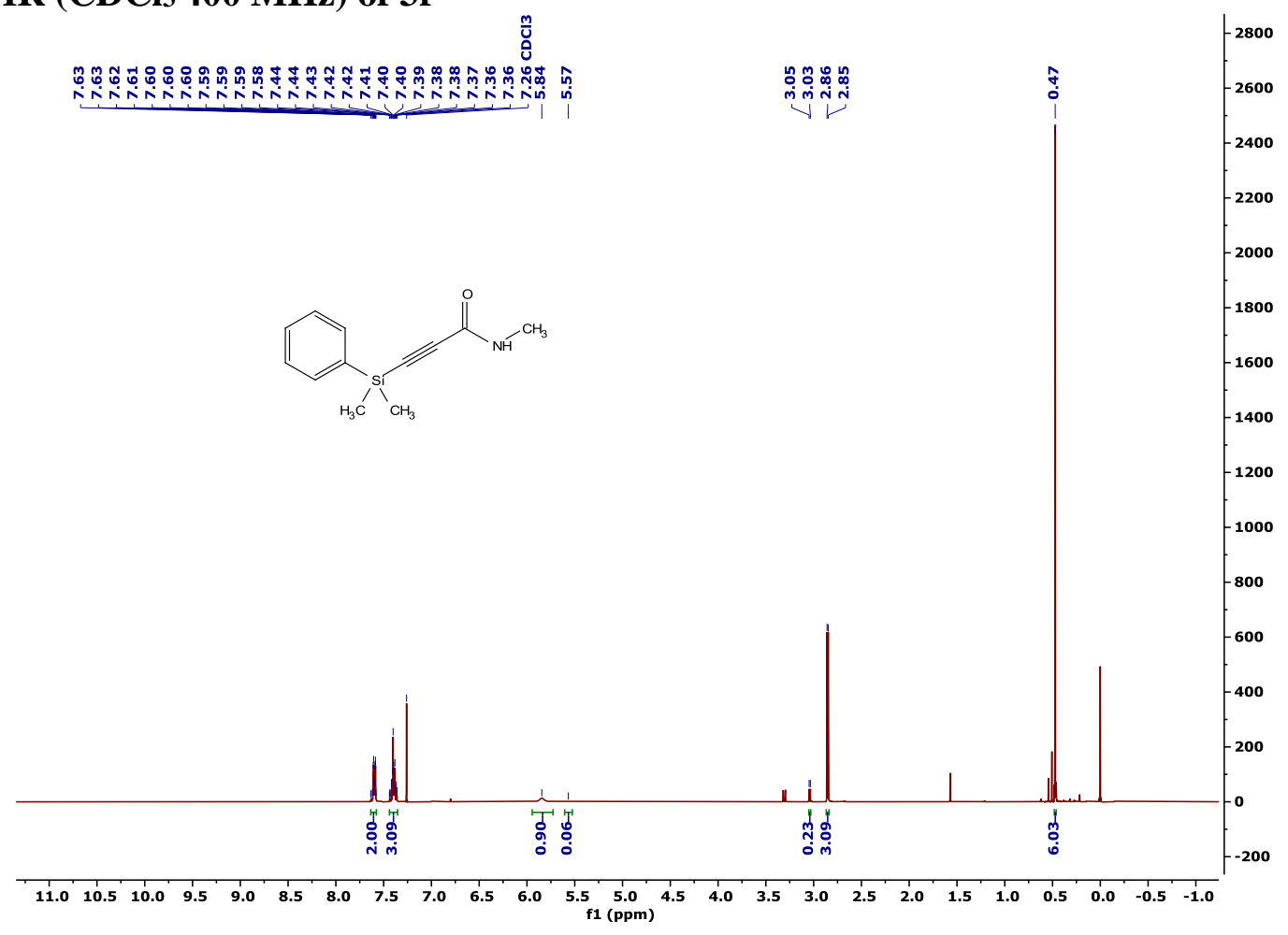


${ }^{13} \mathrm{C} \mathrm{NMR}\left(\mathrm{CDCl}_{3} 100 \mathrm{MHz}\right)$ of $3 \mathrm{f}$

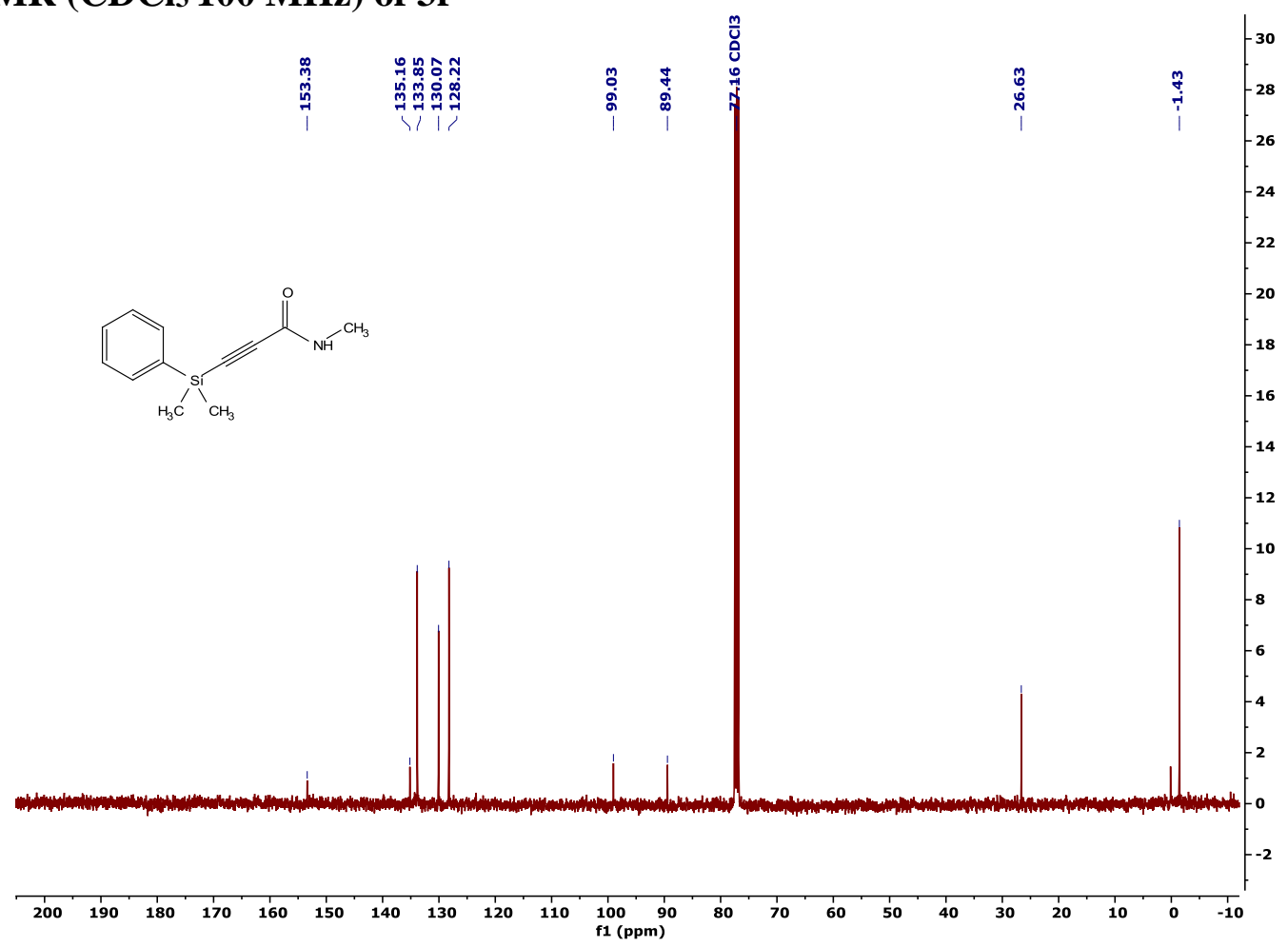

\section{${ }^{1} \mathrm{H}$ NMR (Acetone- $d_{6} 400 \mathrm{MHz}$ ) of $5 \mathrm{~d}$}

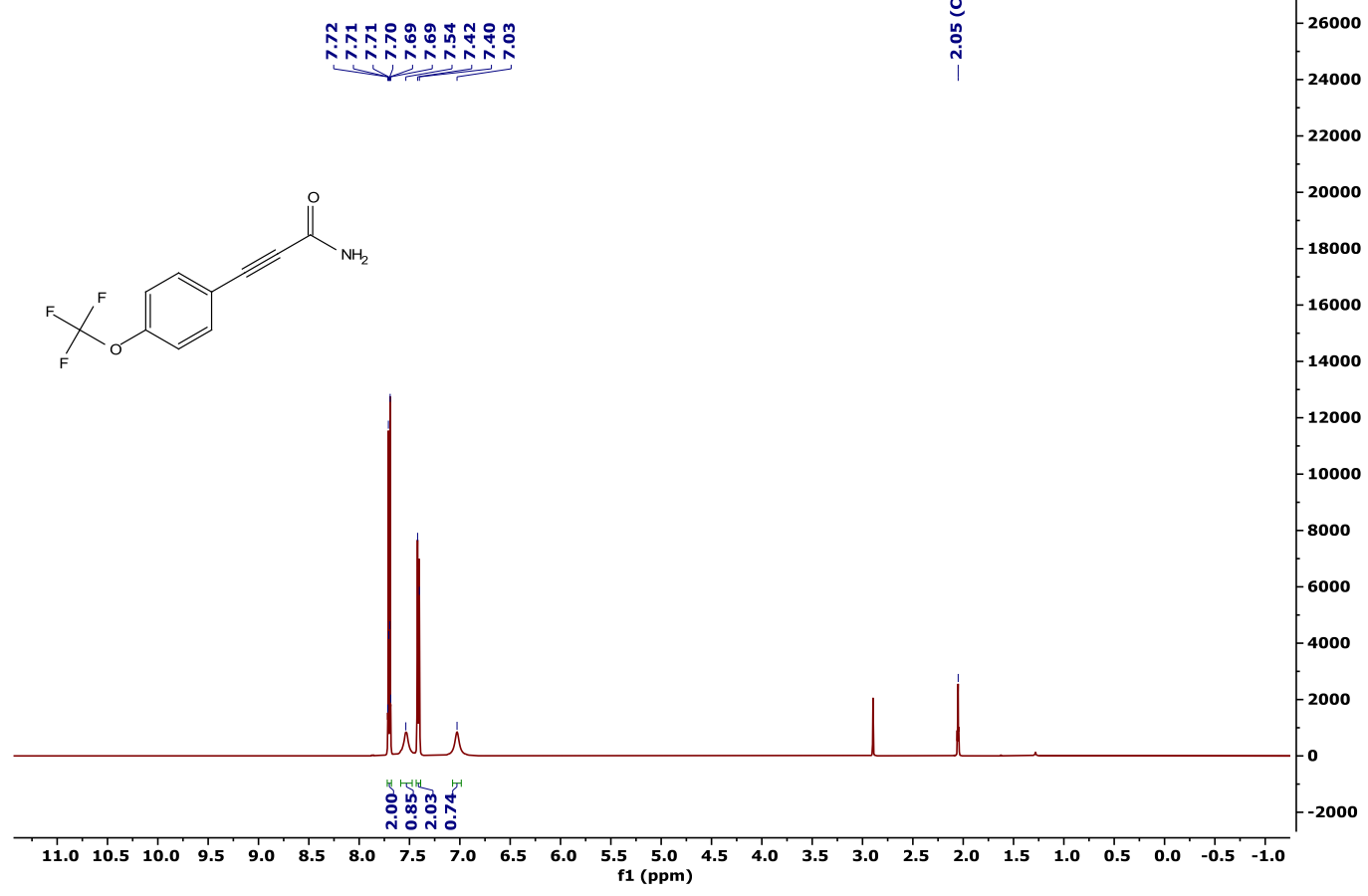


${ }^{19}$ F NMR (Acetone- $d_{6} 376 \mathrm{MHz}$ ) of $5 \mathrm{~d}$

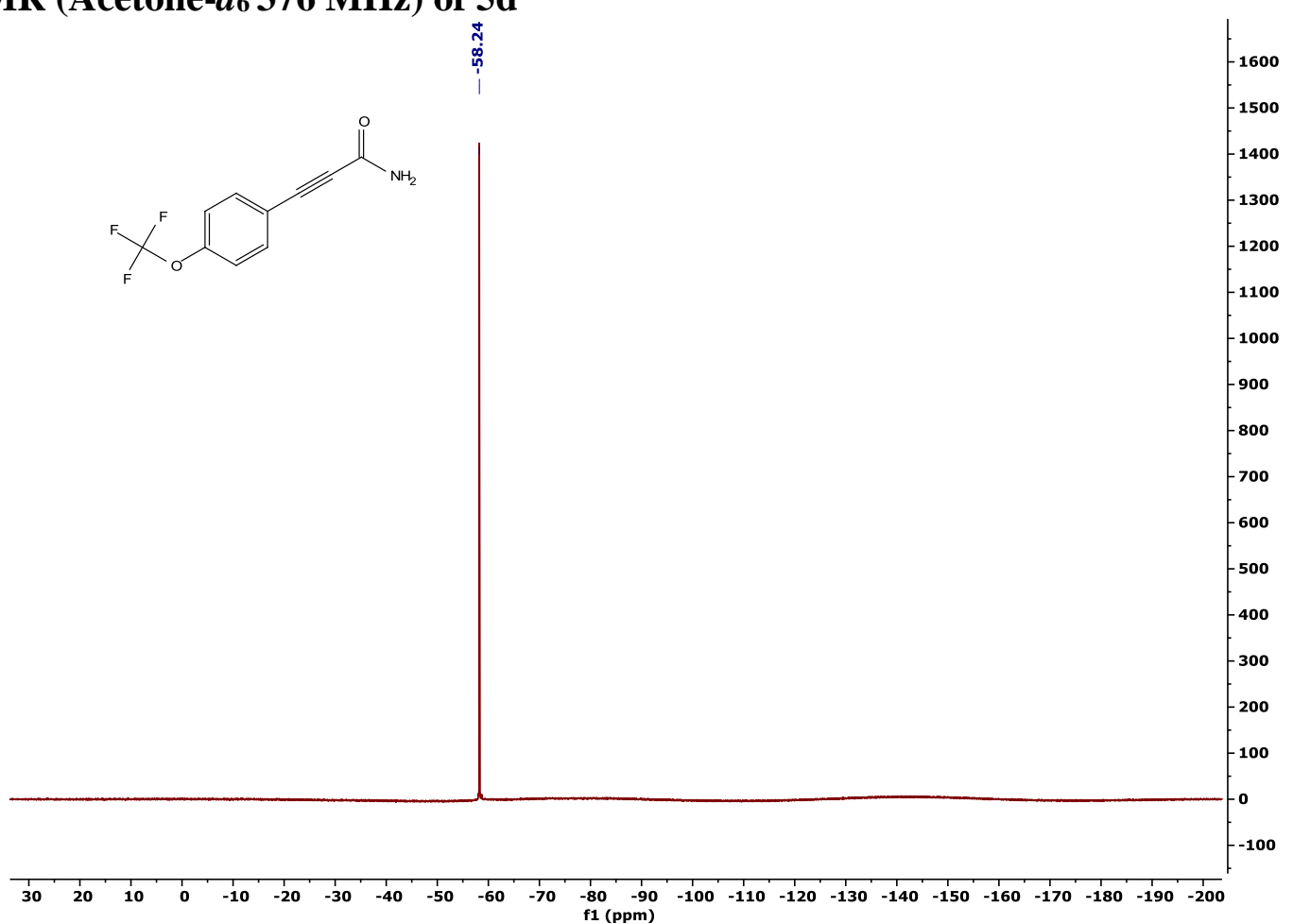

${ }^{13} \mathrm{C}$ NMR (Acetone- $d_{6} 100 \mathrm{MHz}$ ) of $5 \mathrm{~d}$

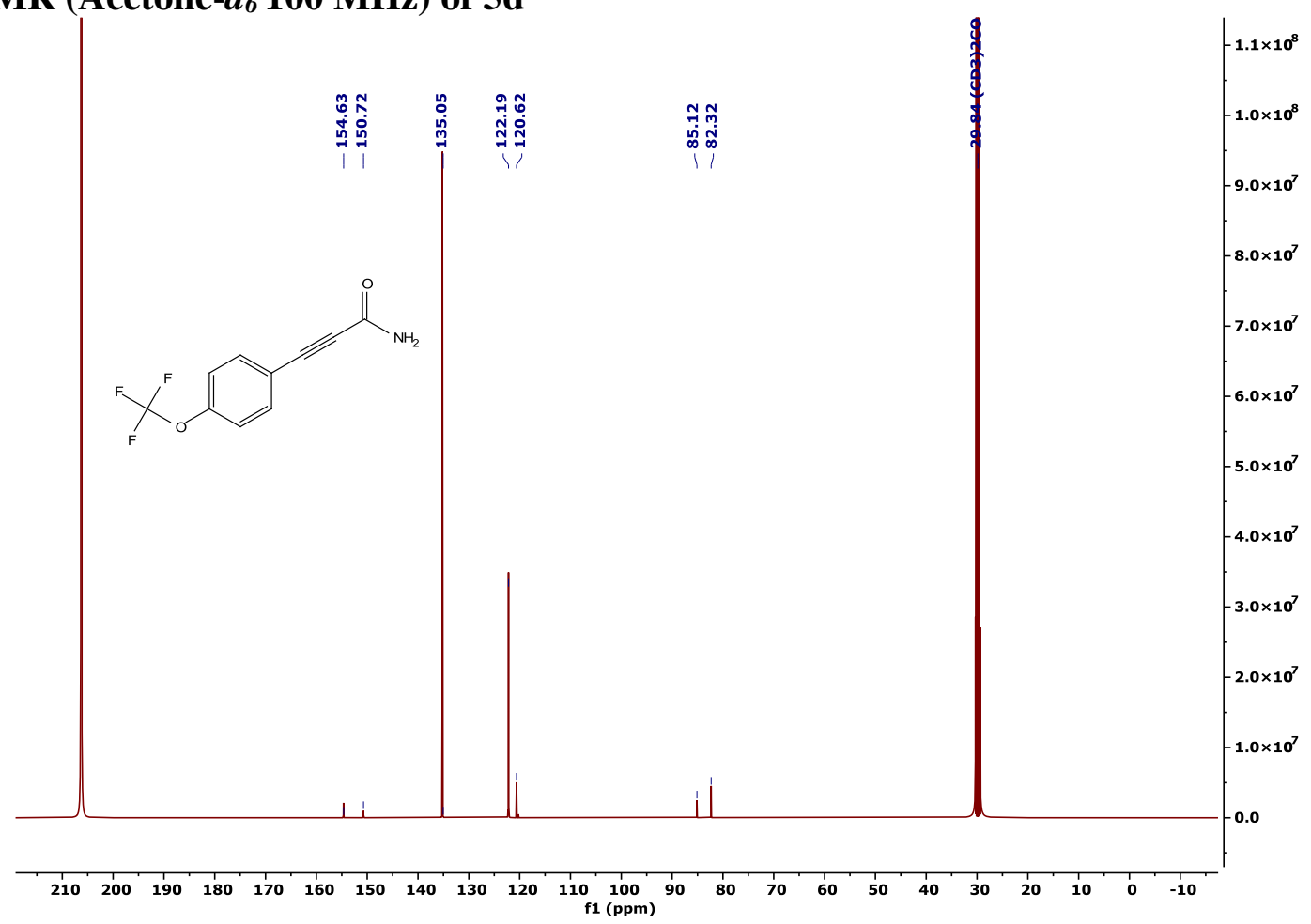


${ }^{1} \mathrm{H}$ NMR (Acetone- $d_{6} 400 \mathrm{MHz}$ ) of $5 \mathrm{e}$

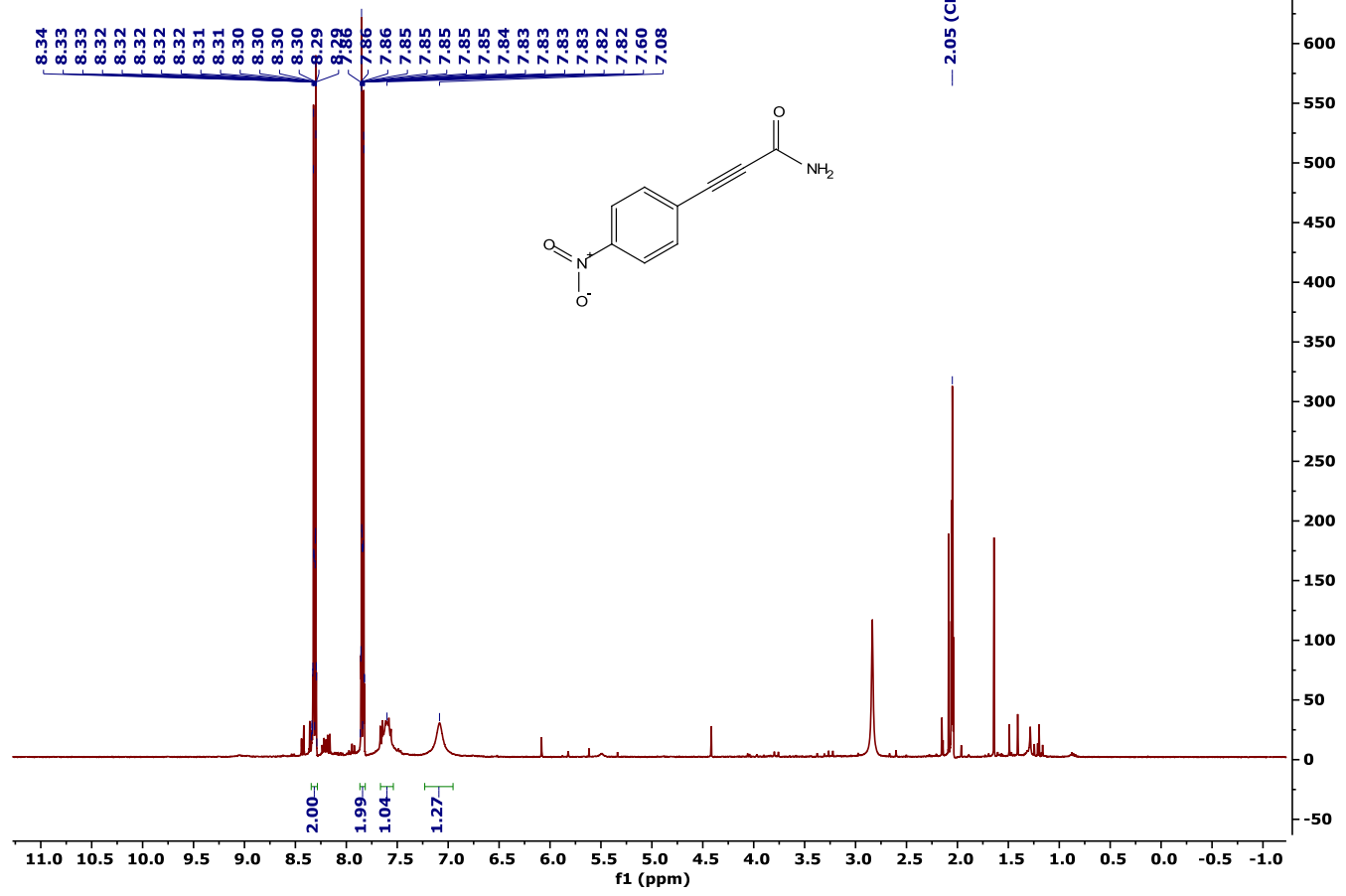

${ }^{13} \mathrm{C}$ NMR (Acetone- $d_{6} 126 \mathrm{MHz}$ ) of $5 \mathrm{e}$

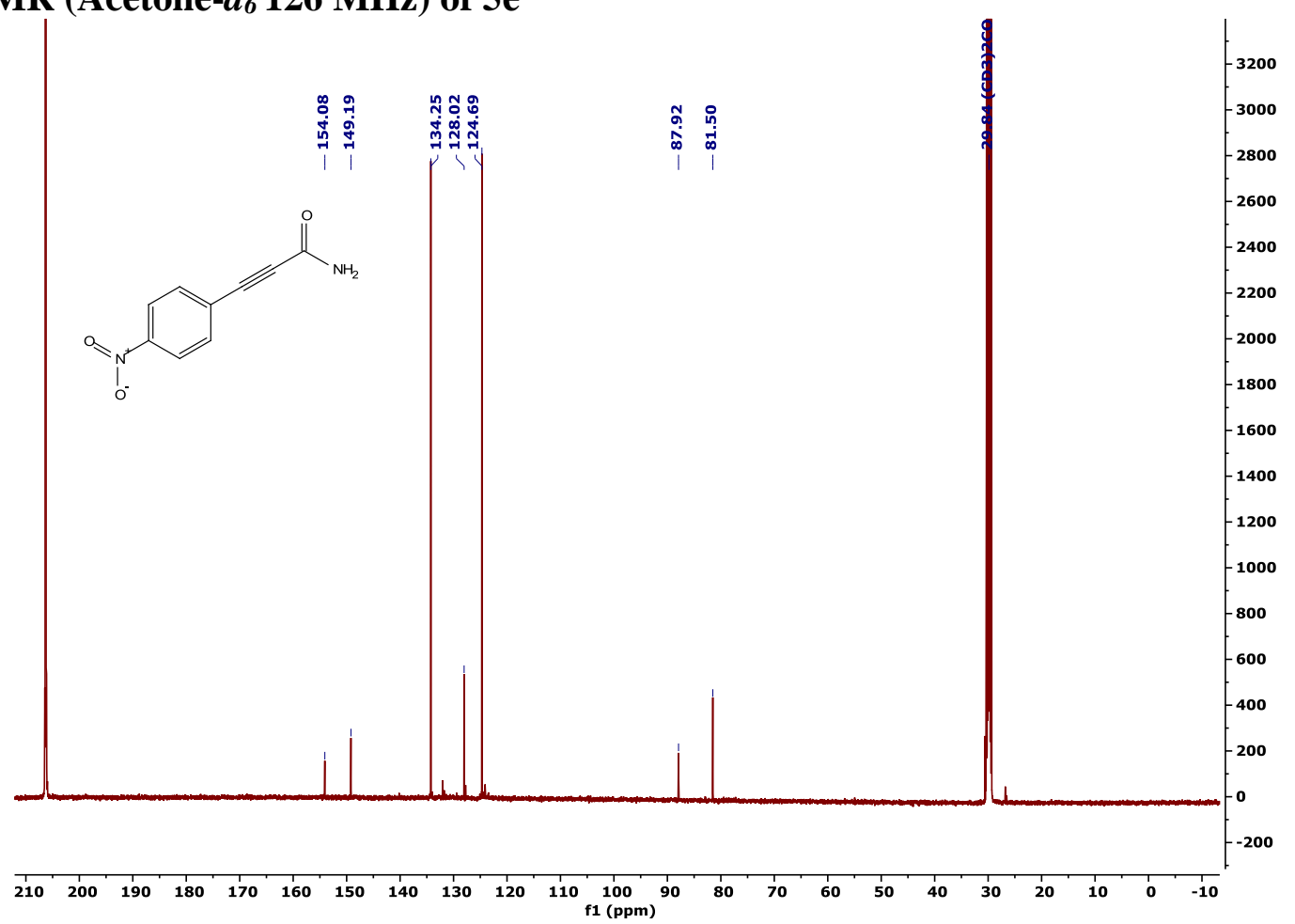


${ }^{1} \mathrm{H} \mathrm{NMR}\left(\mathrm{CD}_{3} \mathrm{OD} 400 \mathrm{MHz}\right)$ of $5 \mathrm{~g}$

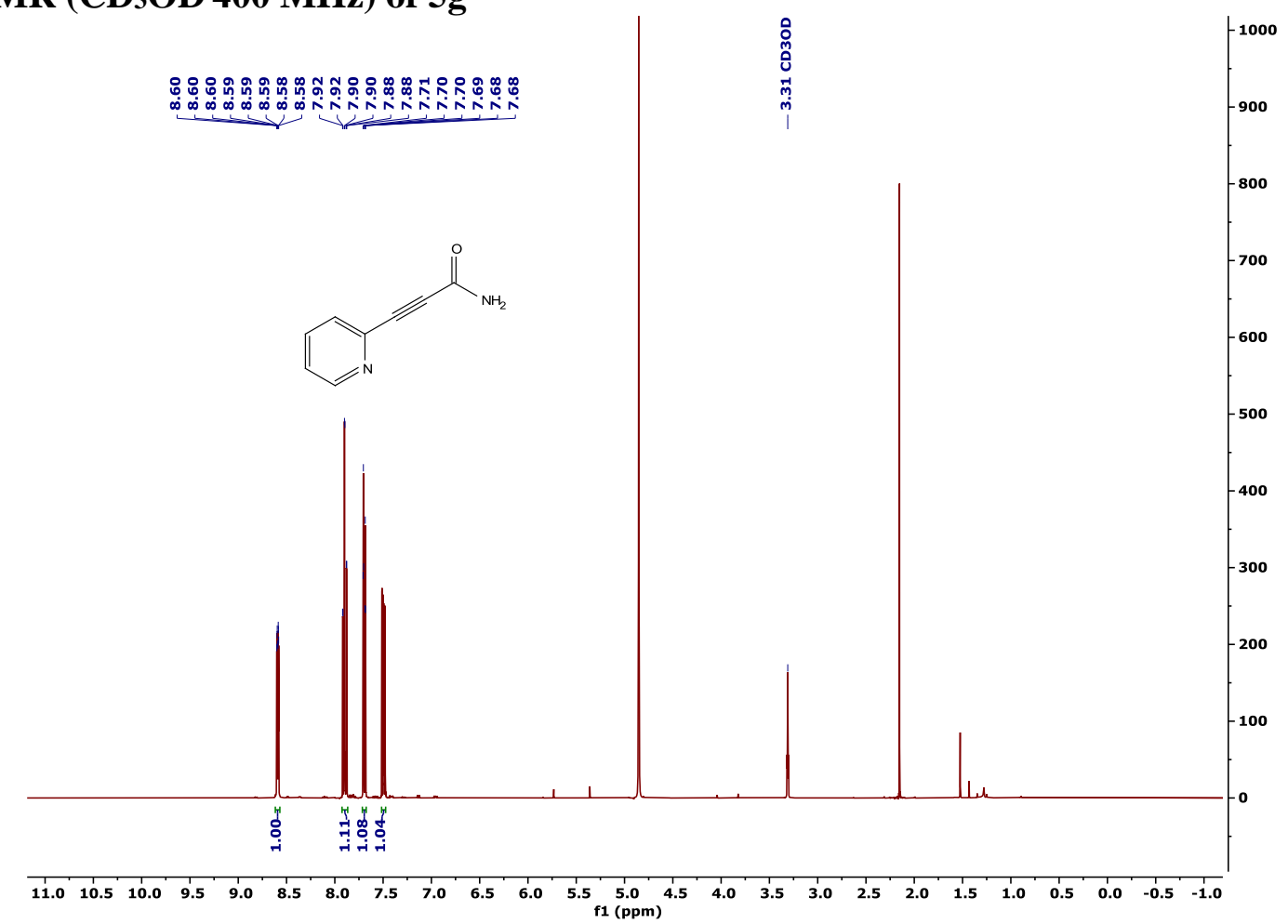

${ }^{13} \mathrm{C}$ NMR (CD3OD $100 \mathrm{MHz}$ ) of $5 \mathrm{~g}$

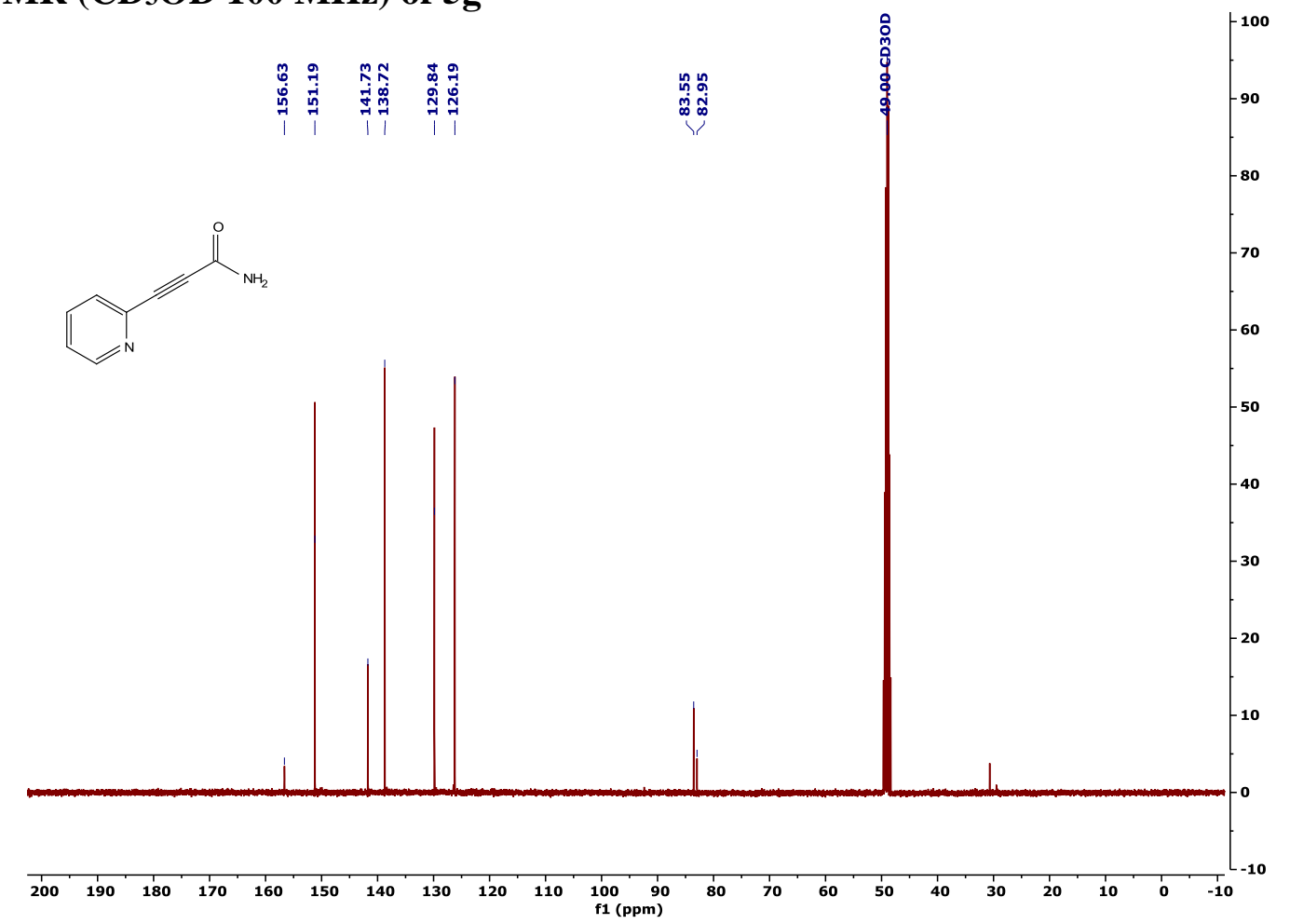


${ }^{1} \mathrm{H}$ NMR (CD $\left.30 D 400 \mathrm{MHz}\right)$ of $5 \mathrm{~h}$

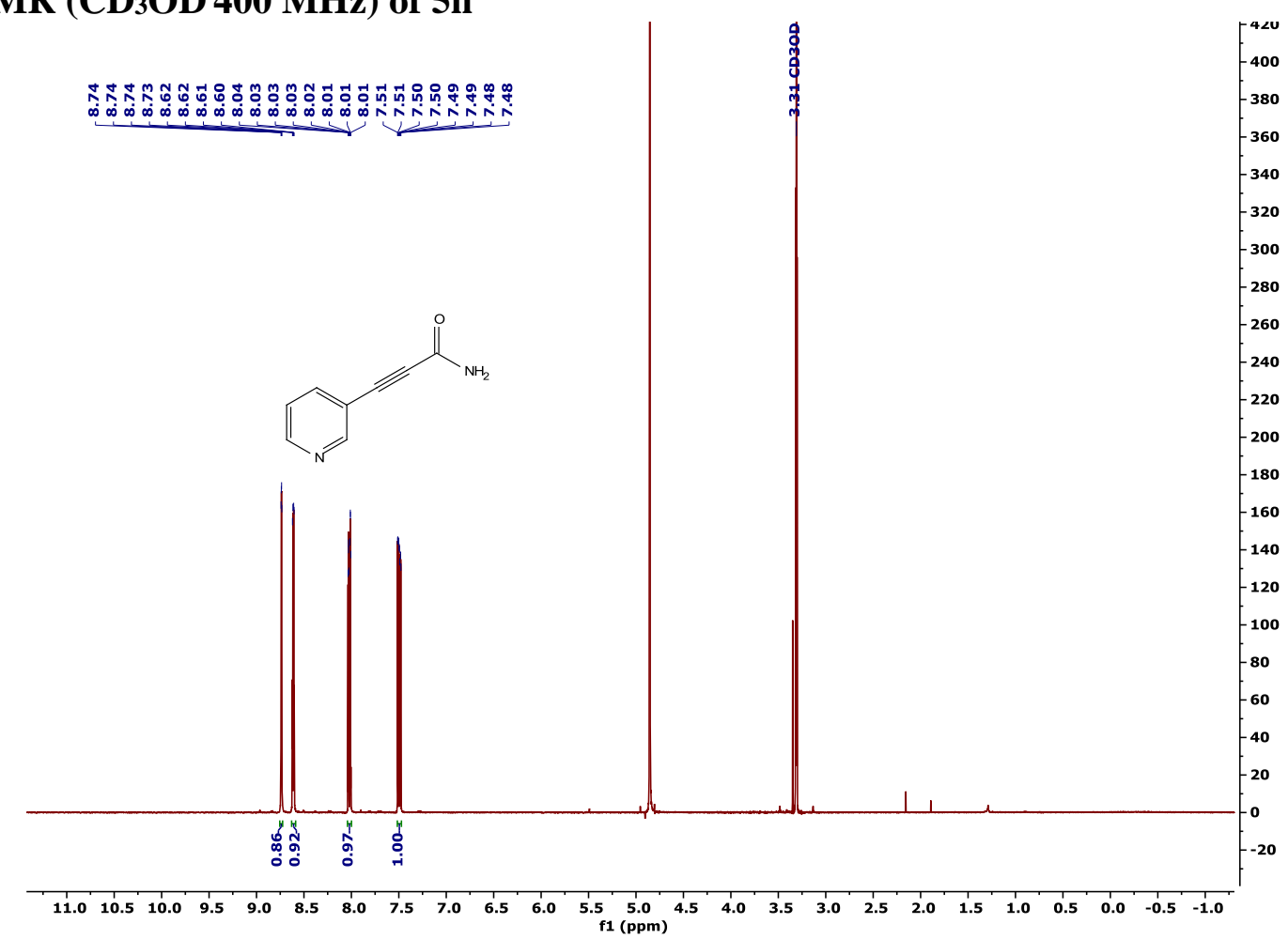

${ }^{13} \mathrm{C} \mathrm{NMR} \mathrm{(CD3OD} 100 \mathrm{MHz}$ ) of $5 \mathrm{~h}$

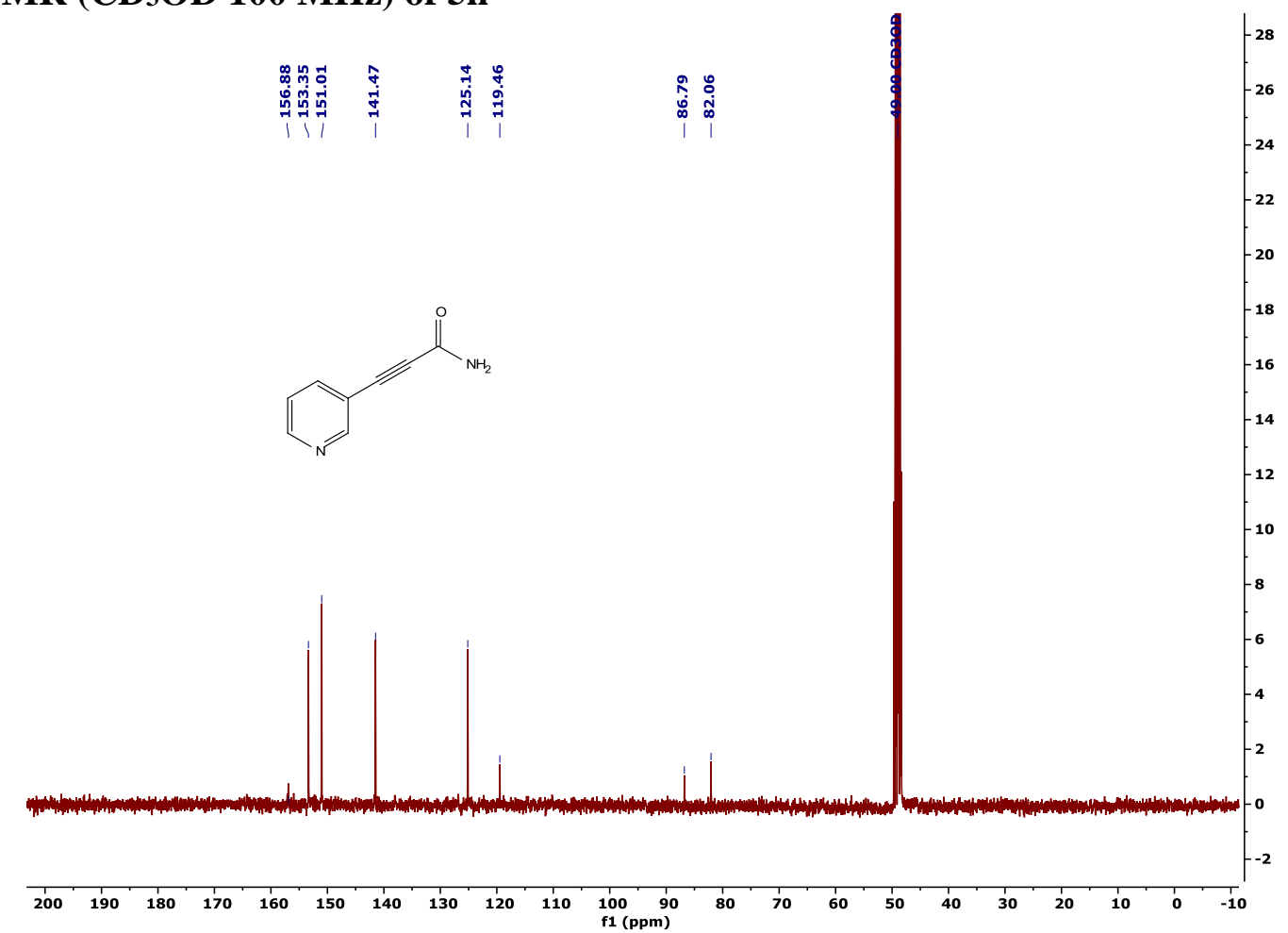


${ }^{1} \mathrm{H}$ NMR $\left(\mathrm{CDCl}_{3} 400 \mathrm{MHz}\right)$ of $5 \mathrm{i}$

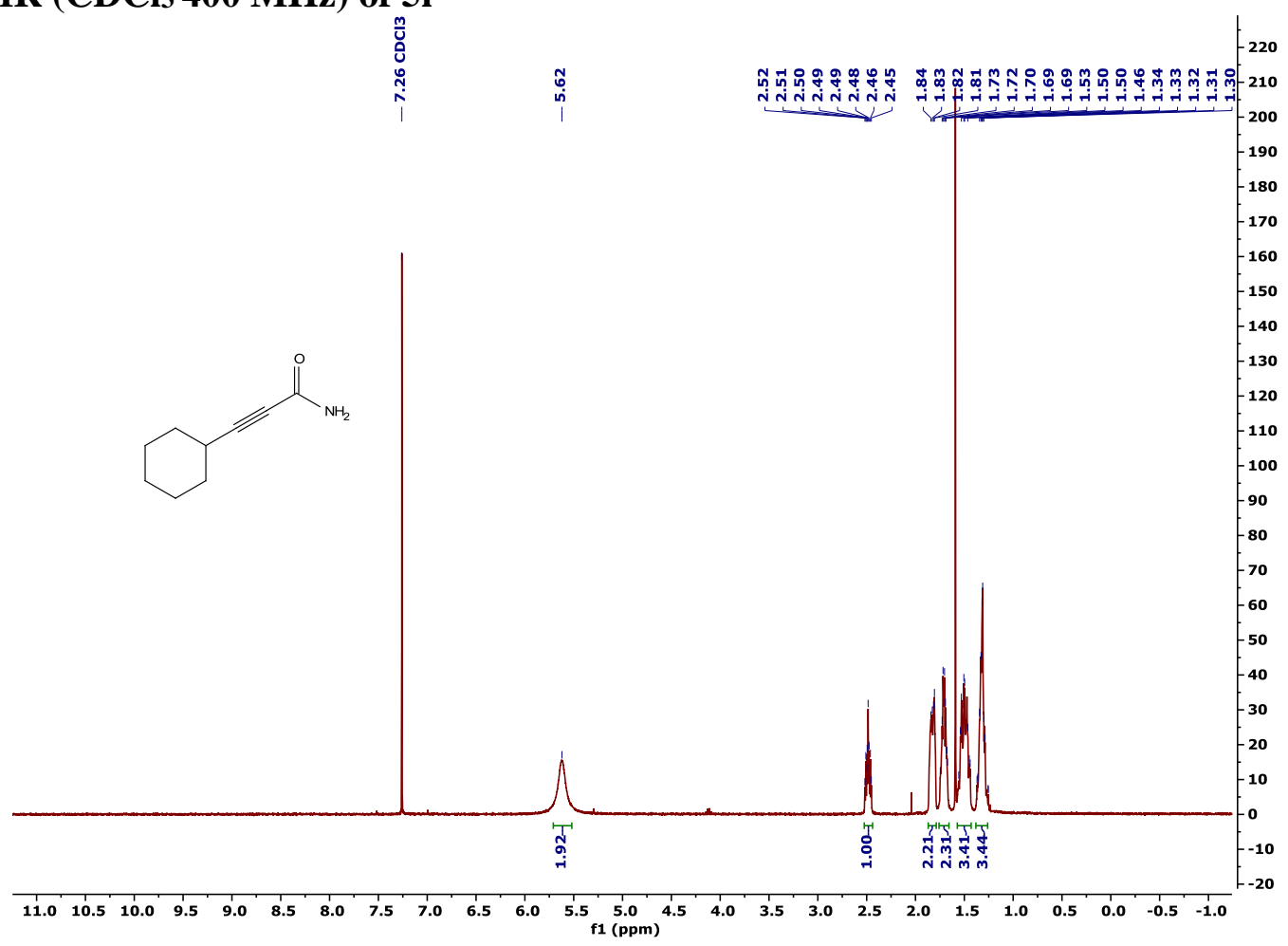

${ }^{13} \mathrm{C}$ NMR $\left(\mathrm{CDCl}_{3} 100 \mathrm{MHz}\right)$ of $5 \mathrm{i}$

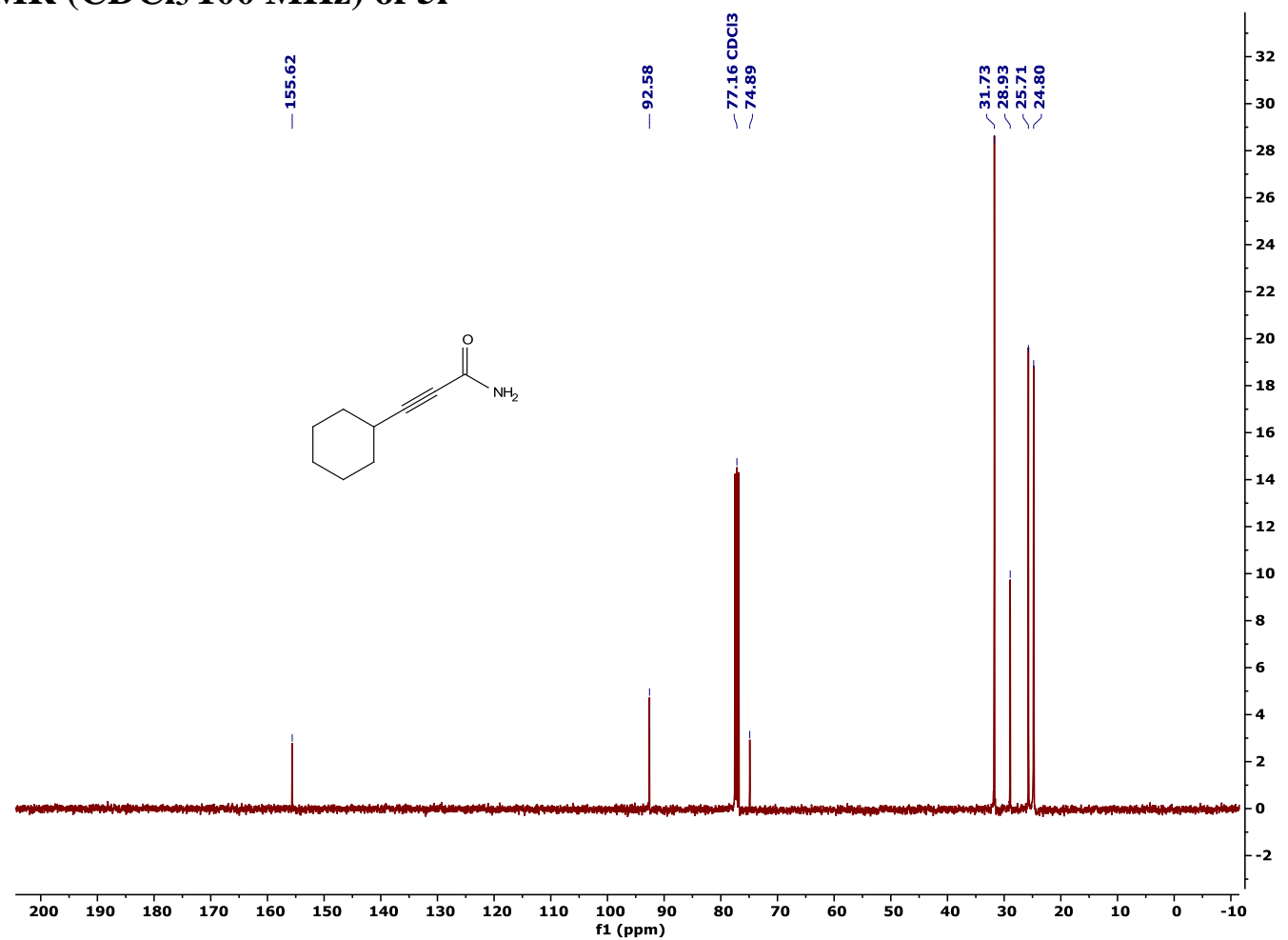


12. NMR Spectra of $(E)$-Cinnamamides

${ }^{1} \mathrm{H}$ NMR $\left(\mathrm{CDCl}_{3} 400 \mathrm{MHz}\right)$ of $2 \mathrm{a}$

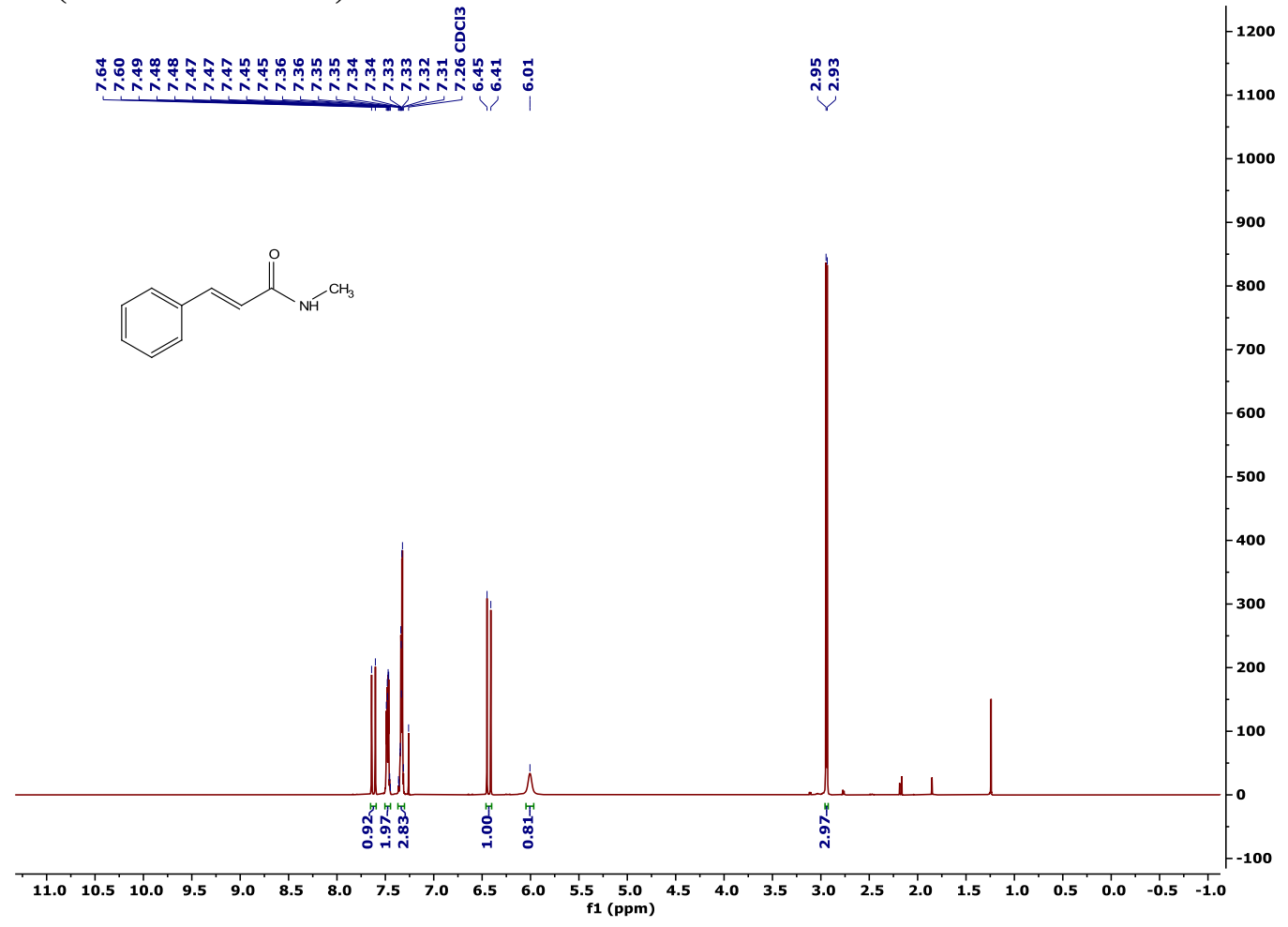

${ }^{13} \mathrm{C} \mathrm{NMR}\left(\mathrm{CDCl}_{3} 100 \mathrm{MHz}\right)$ of $2 \mathrm{a}$

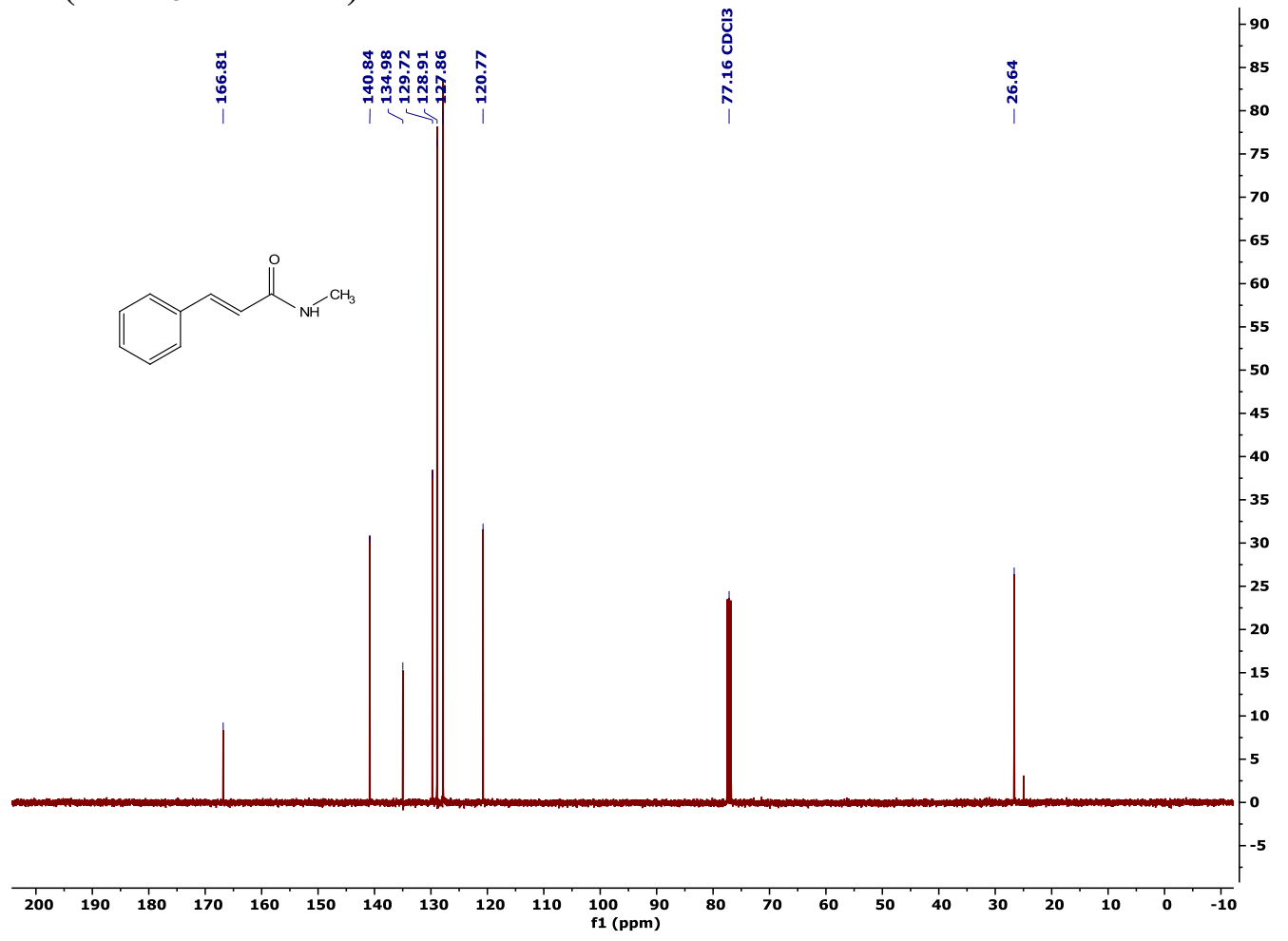


${ }^{1} \mathrm{H}$ NMR $\left(\mathrm{CDCl}_{3} 400 \mathrm{MHz}\right)$ of $2 \mathrm{~b}$

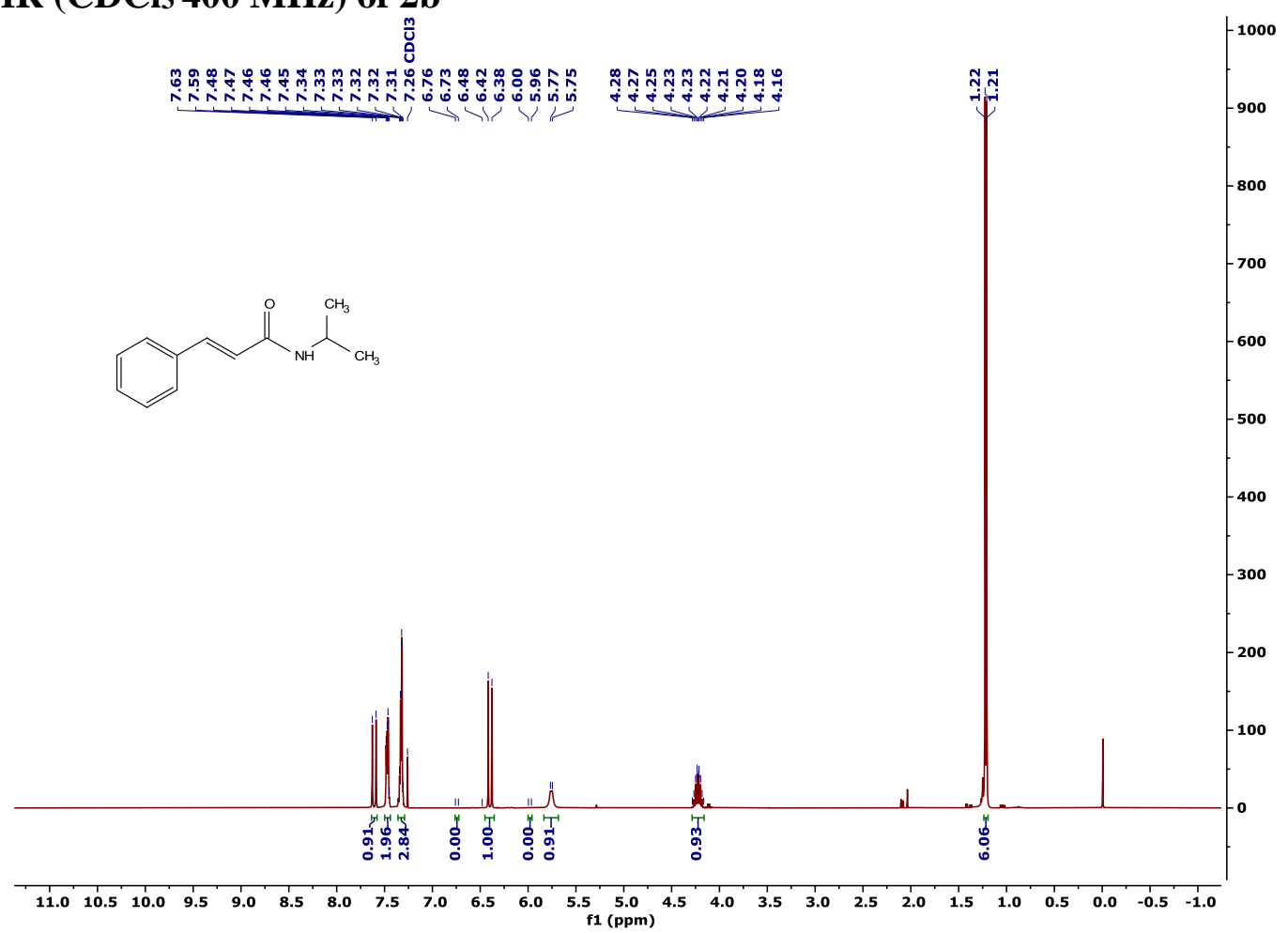

$\left.{ }^{13} \mathrm{C} \mathrm{NMR} \mathrm{(CDCl} 3100 \mathrm{MHz}\right)$ of $2 \mathrm{~b}$

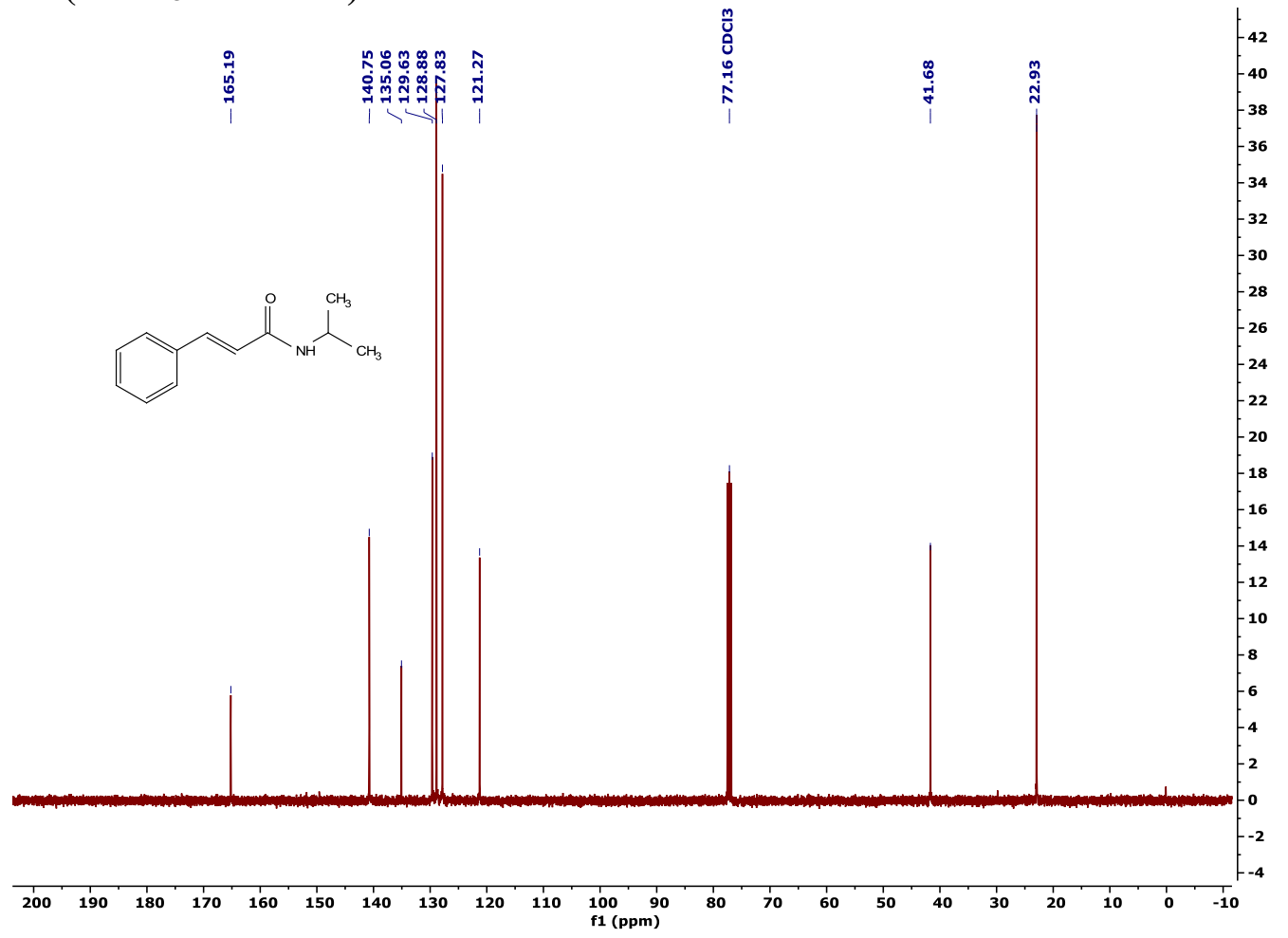


${ }^{1} \mathrm{H}$ NMR ( $\left.\mathrm{CDCl}_{3} 400 \mathrm{MHz}\right)$ of $2 \mathrm{c}$

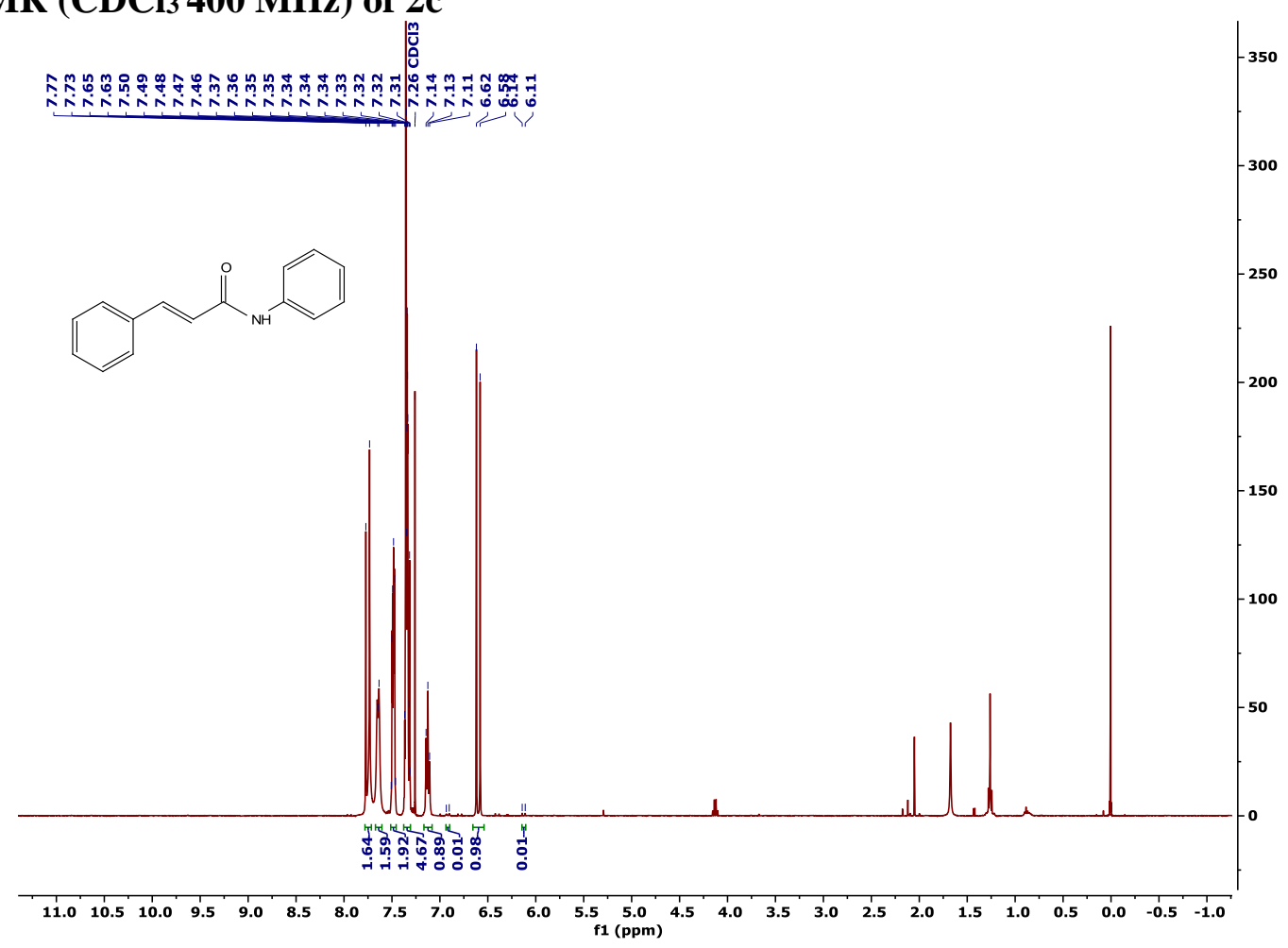

${ }^{13} \mathrm{C}$ NMR (CDCl $\left.3100 \mathrm{MHz}\right)$ of $2 \mathrm{c}$

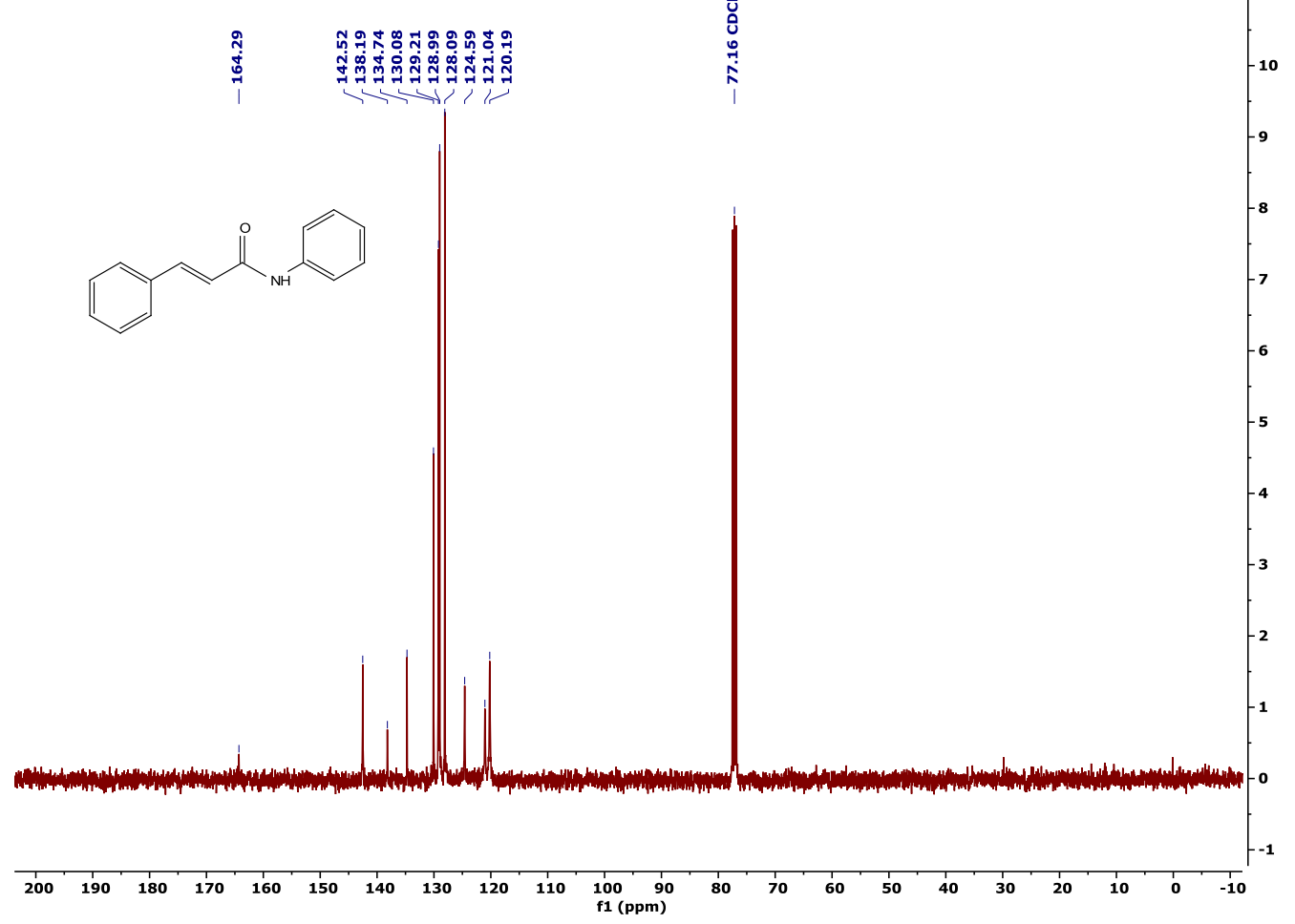


${ }^{1} \mathrm{H}$ NMR $\left(\mathrm{CDCl}_{3} 400 \mathrm{MHz}\right)$ of $2 \mathrm{~d}$

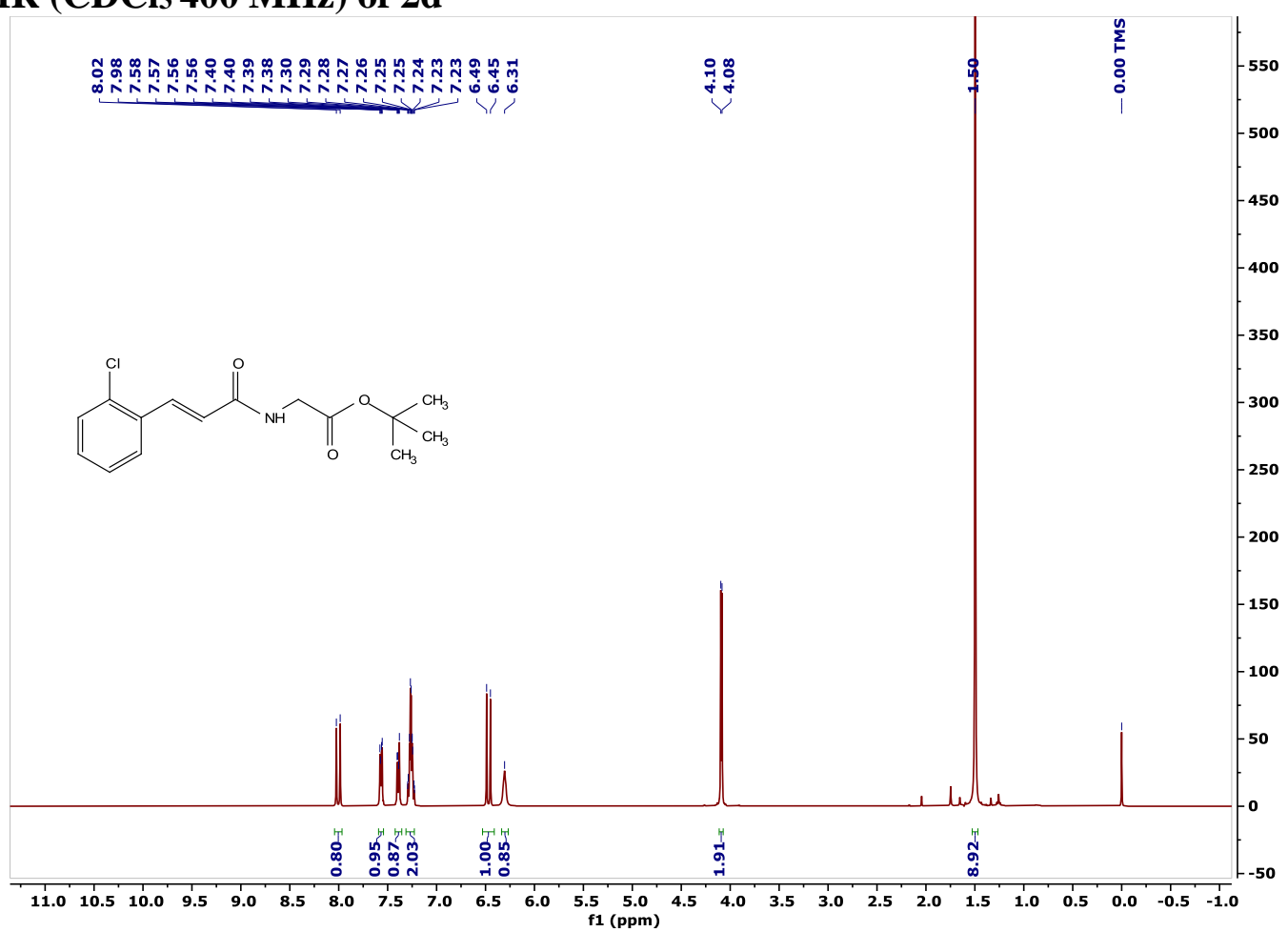

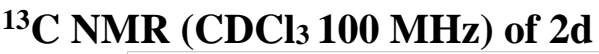

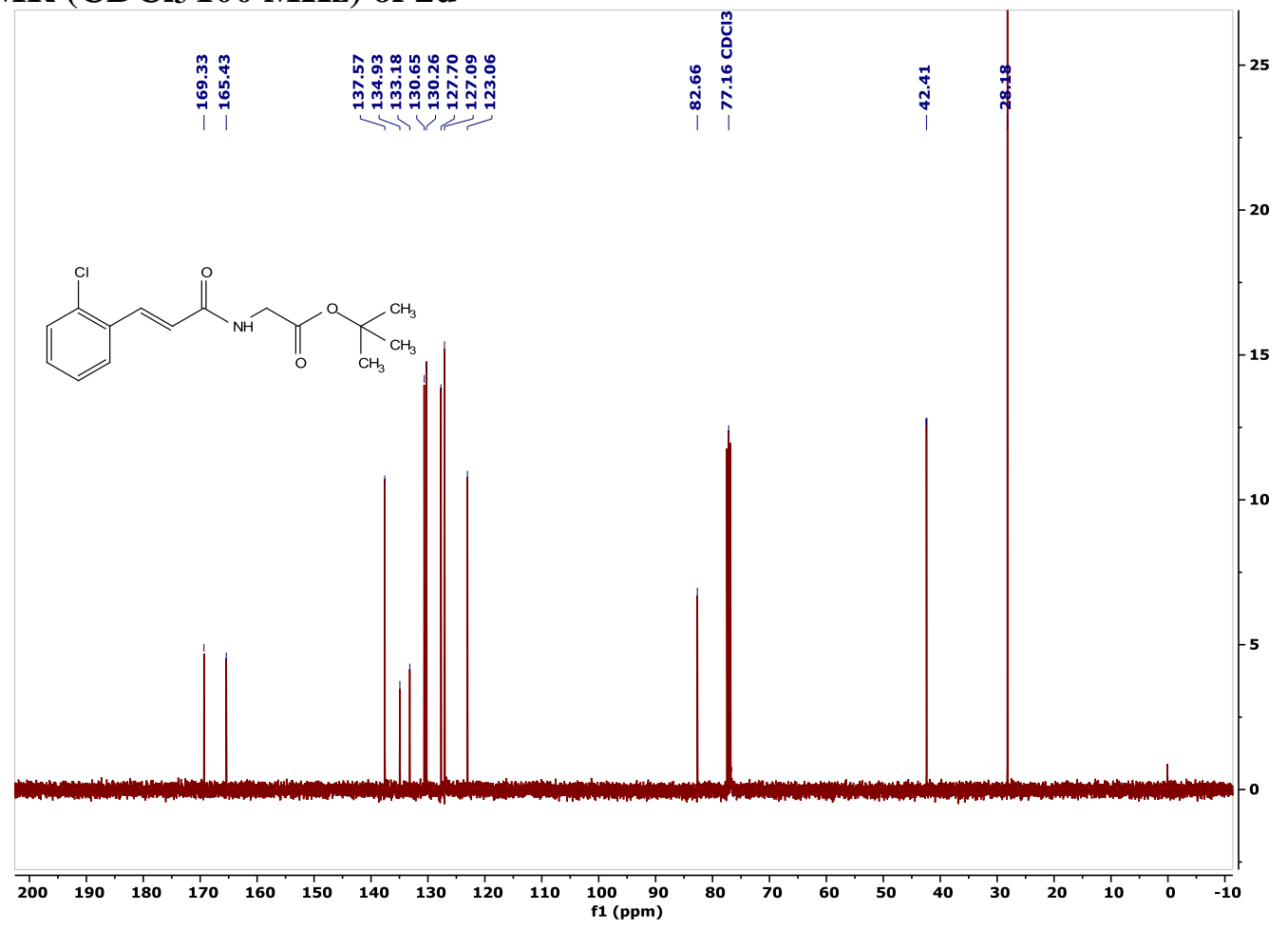


${ }^{1} \mathrm{H}$ NMR ( $\left.\mathrm{CDCl}_{3} 400 \mathrm{MHz}\right)$ of $2 \mathrm{e}$

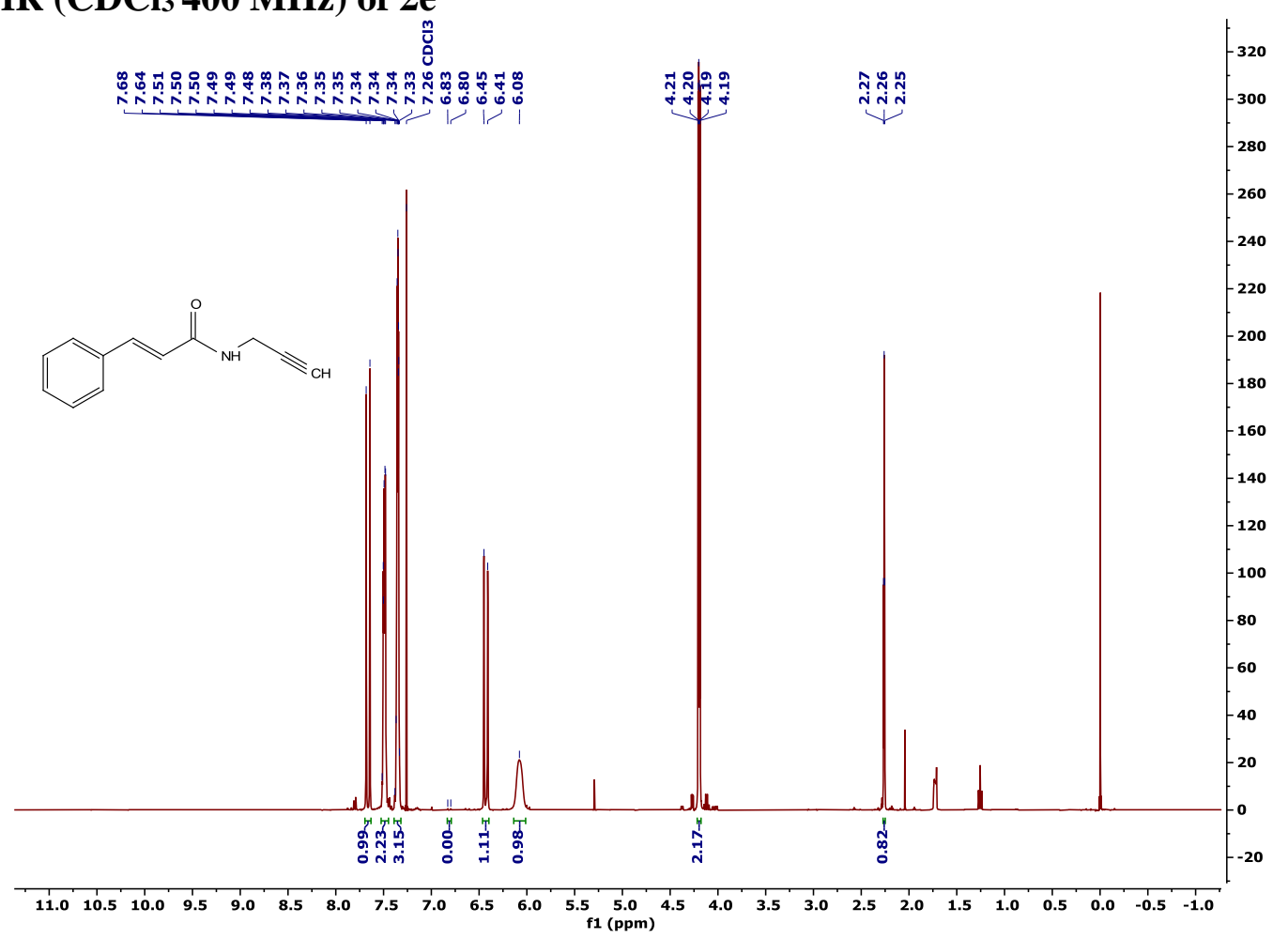

${ }^{13} \mathrm{C} \mathrm{NMR}\left(\mathrm{CDCl}_{3}, 100 \mathrm{MHz}\right)$ of $2 \mathrm{e}$

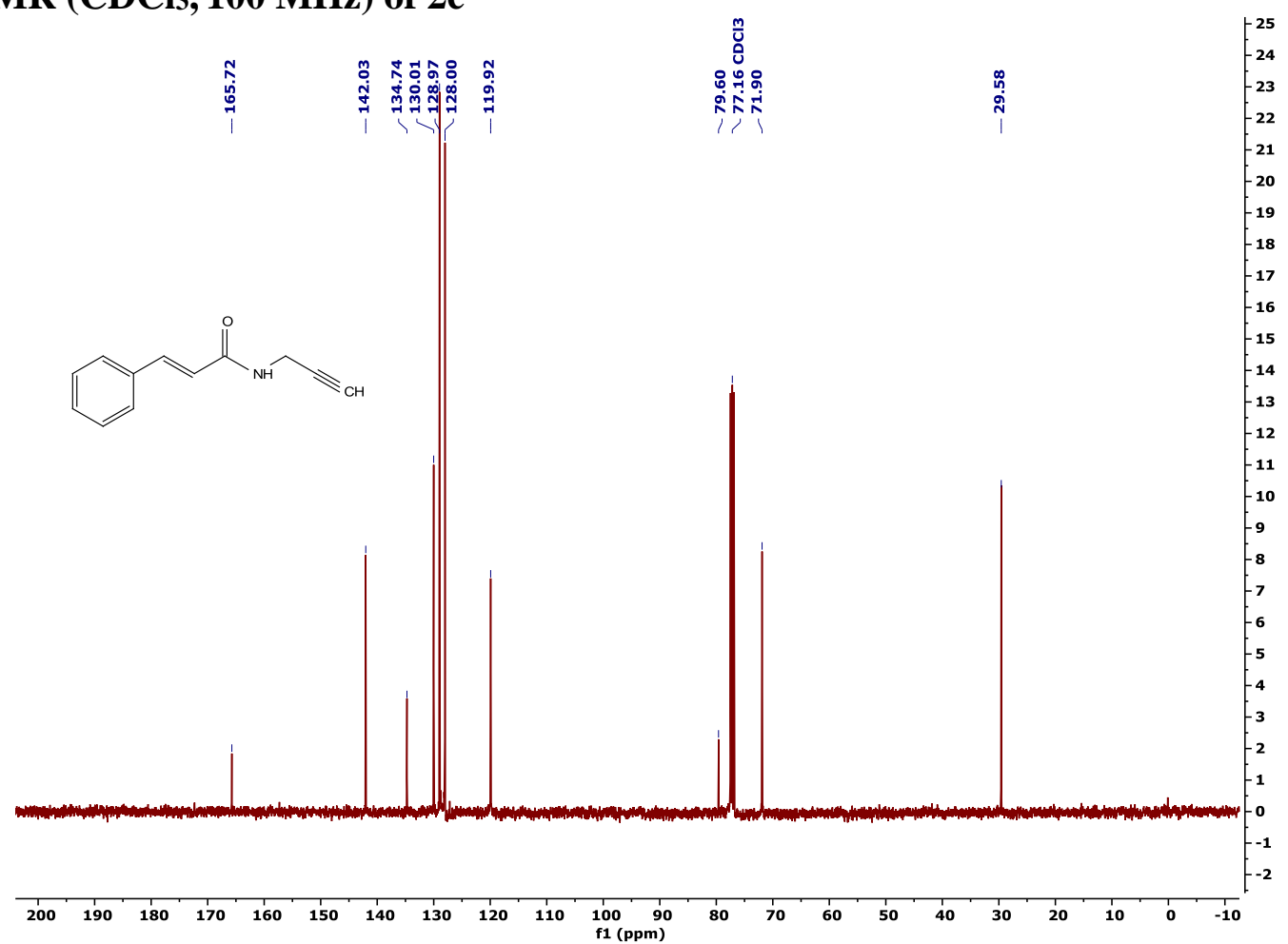


${ }^{1} \mathrm{H}$ NMR ( $\left.\mathrm{CDCl}_{3} 400 \mathrm{MHz}\right)$ of $2 \mathrm{f}$

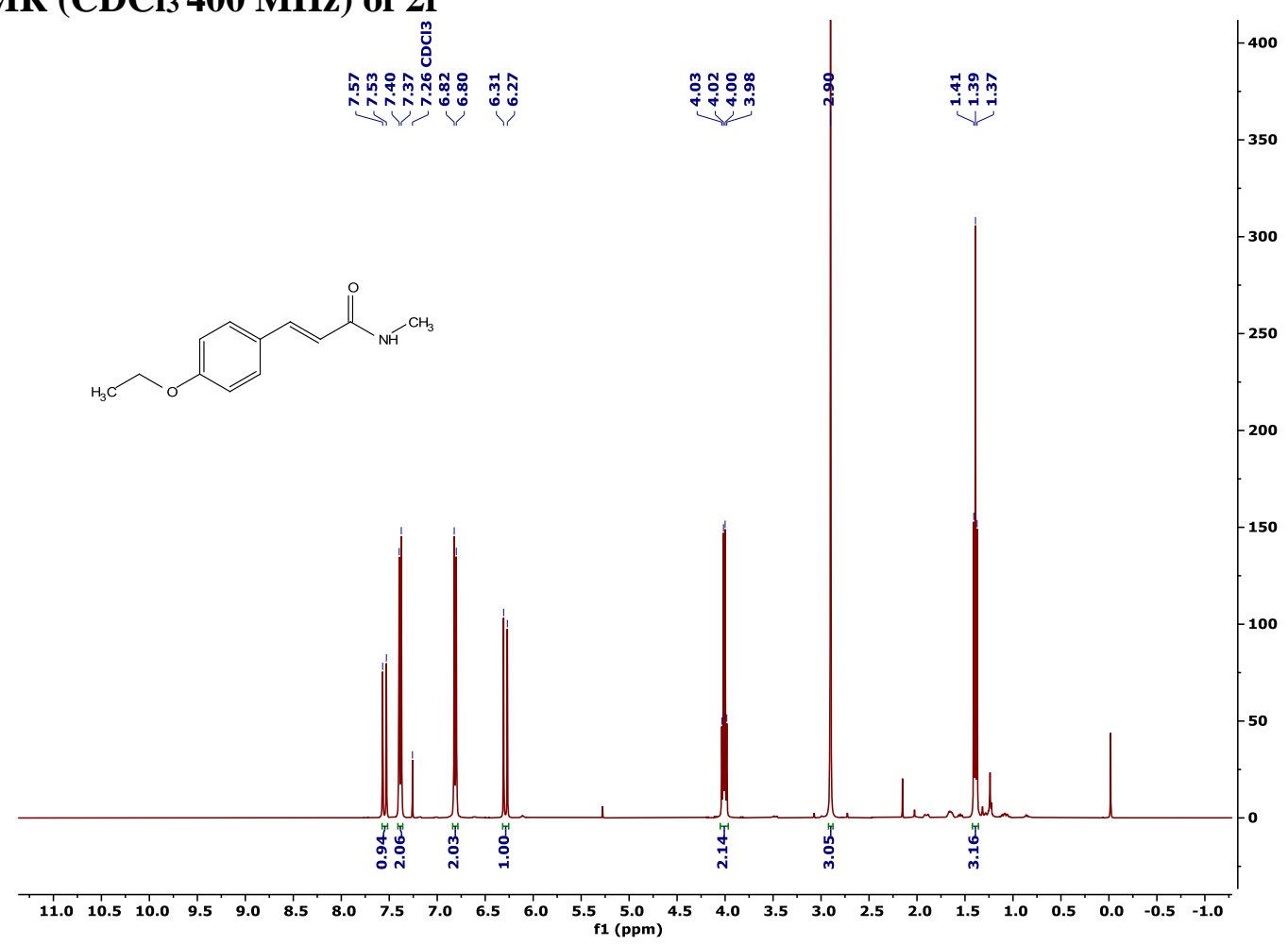

${ }^{13} \mathrm{C}$ NMR (CDCl $3100 \mathrm{MHz}$ ) of $2 \mathrm{f}$

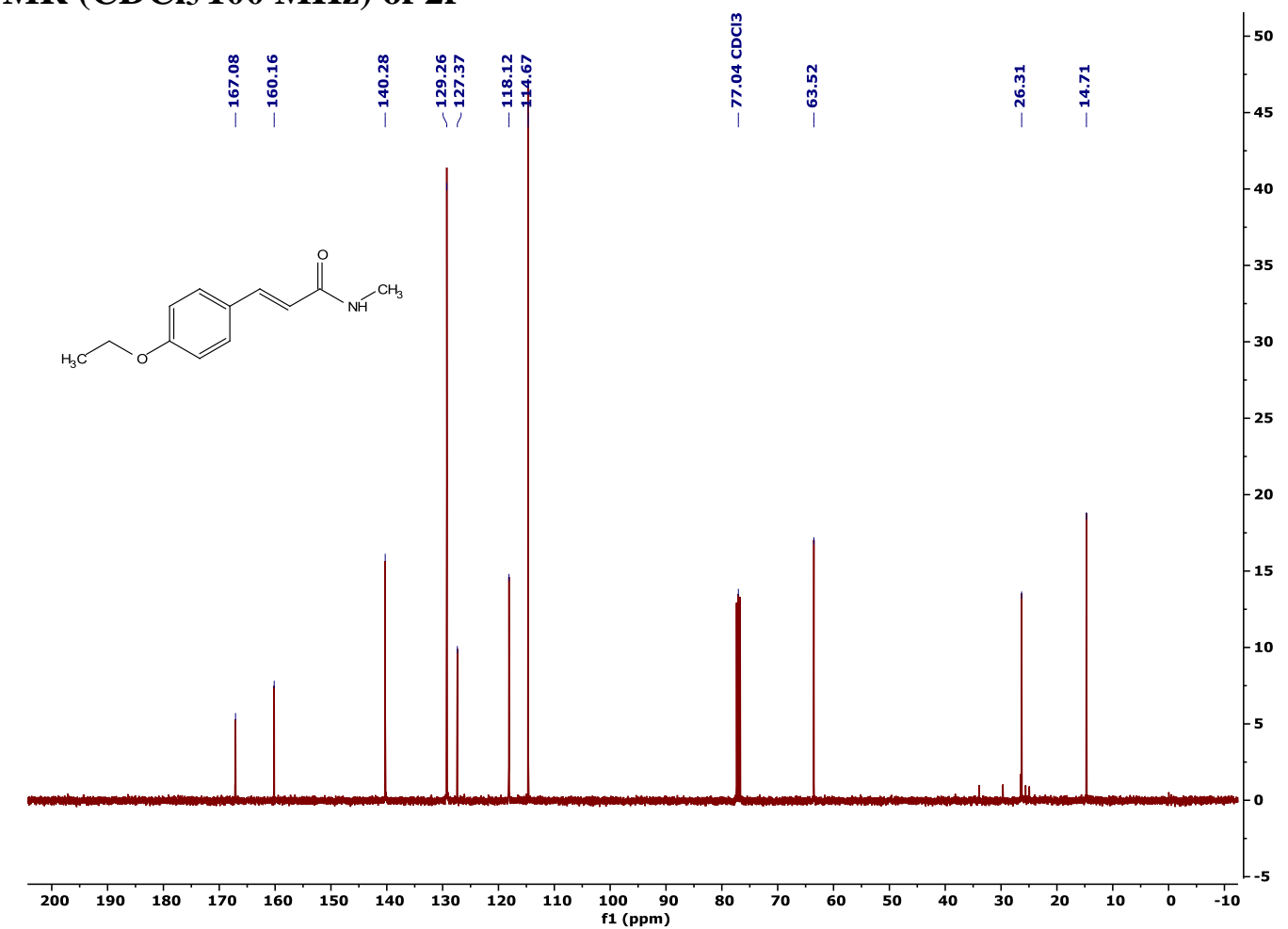


${ }^{1} \mathrm{H}$ NMR $\left(\mathrm{CDCl}_{3} 400 \mathrm{MHz}\right)$ of $2 \mathrm{~g}$

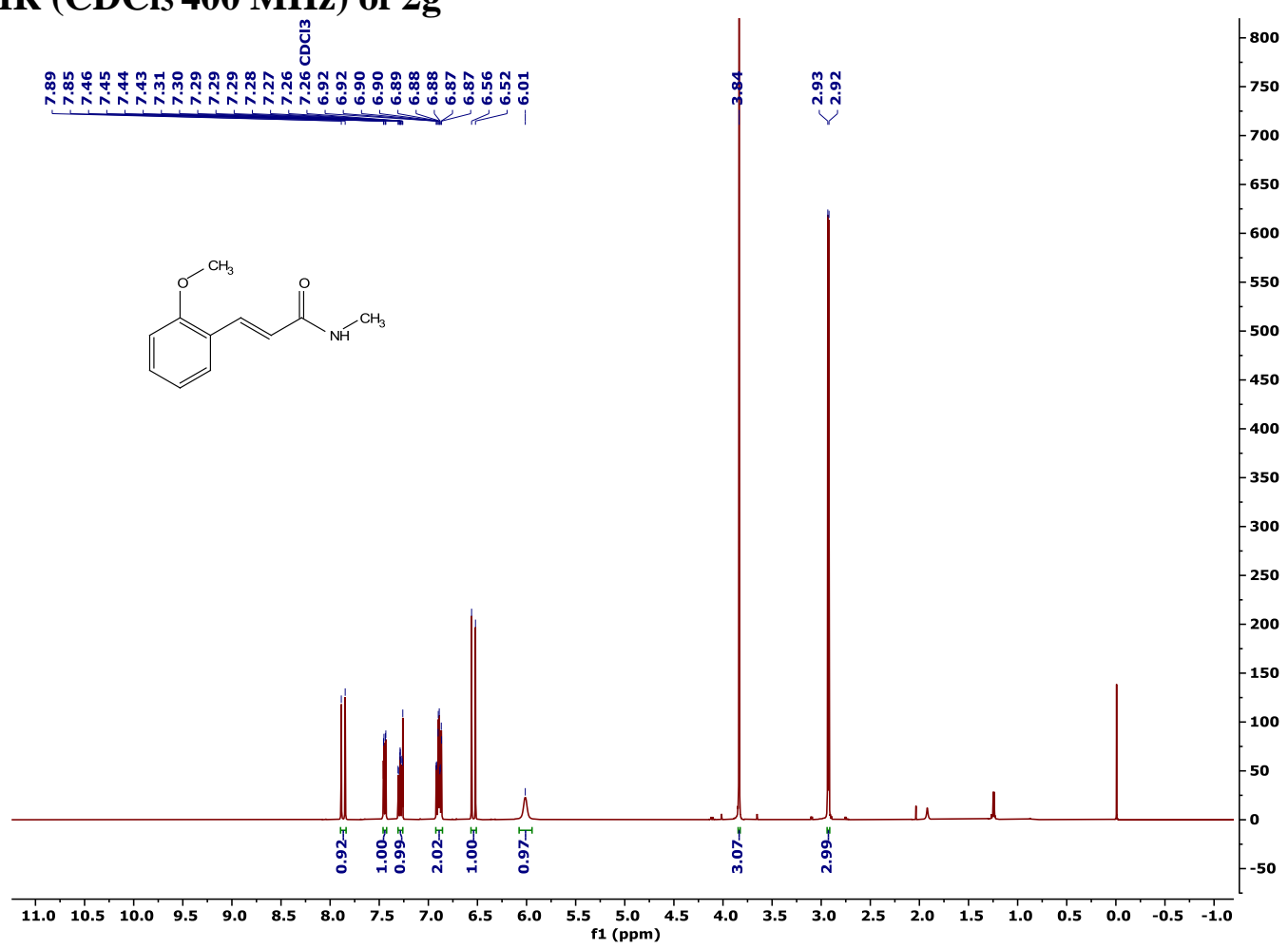

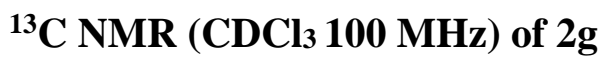

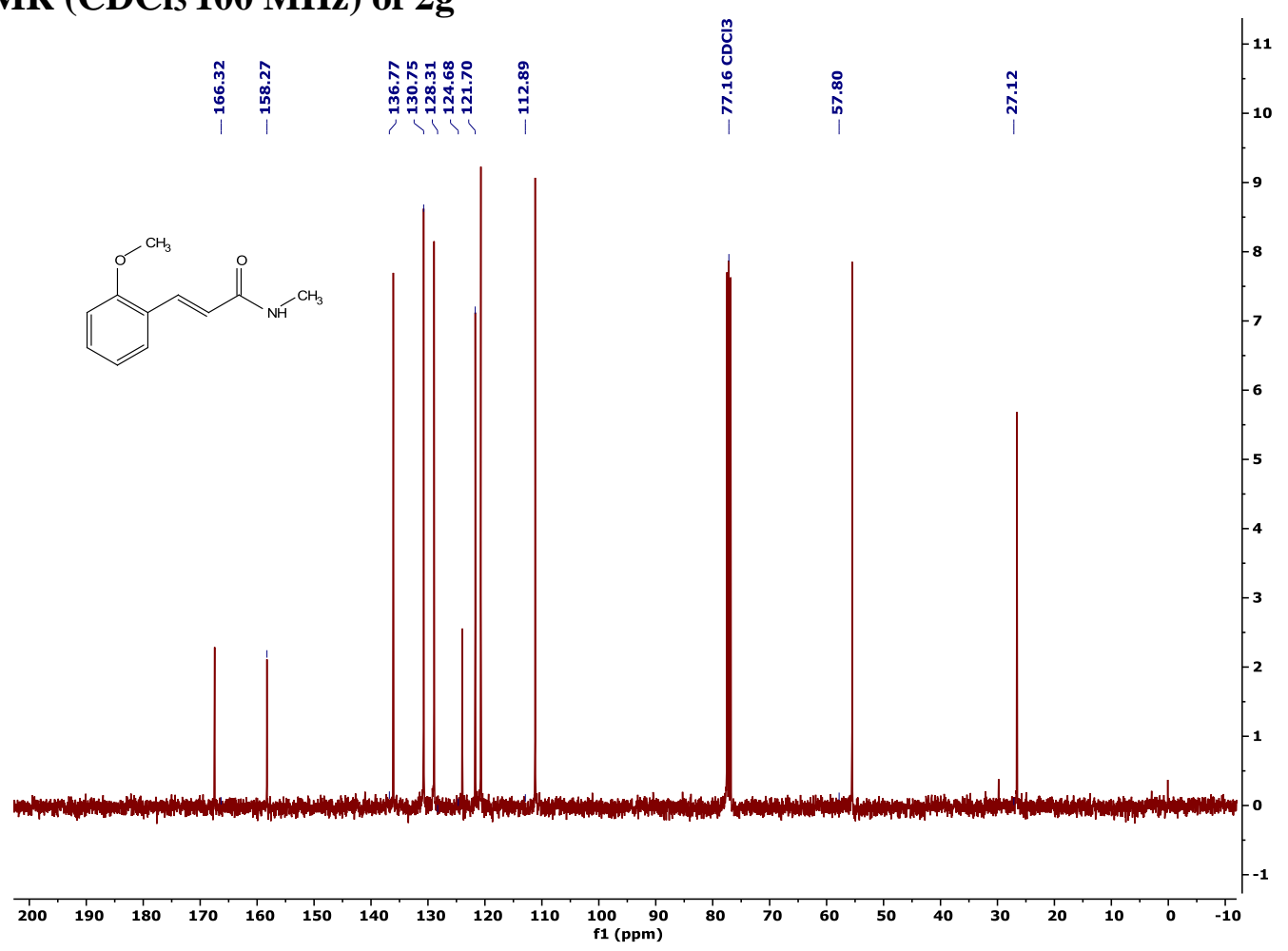


${ }^{1} \mathrm{H}$ NMR $\left(\mathrm{CDCl}_{3} 400 \mathrm{MHz}\right)$ of $2 \mathrm{~h}$

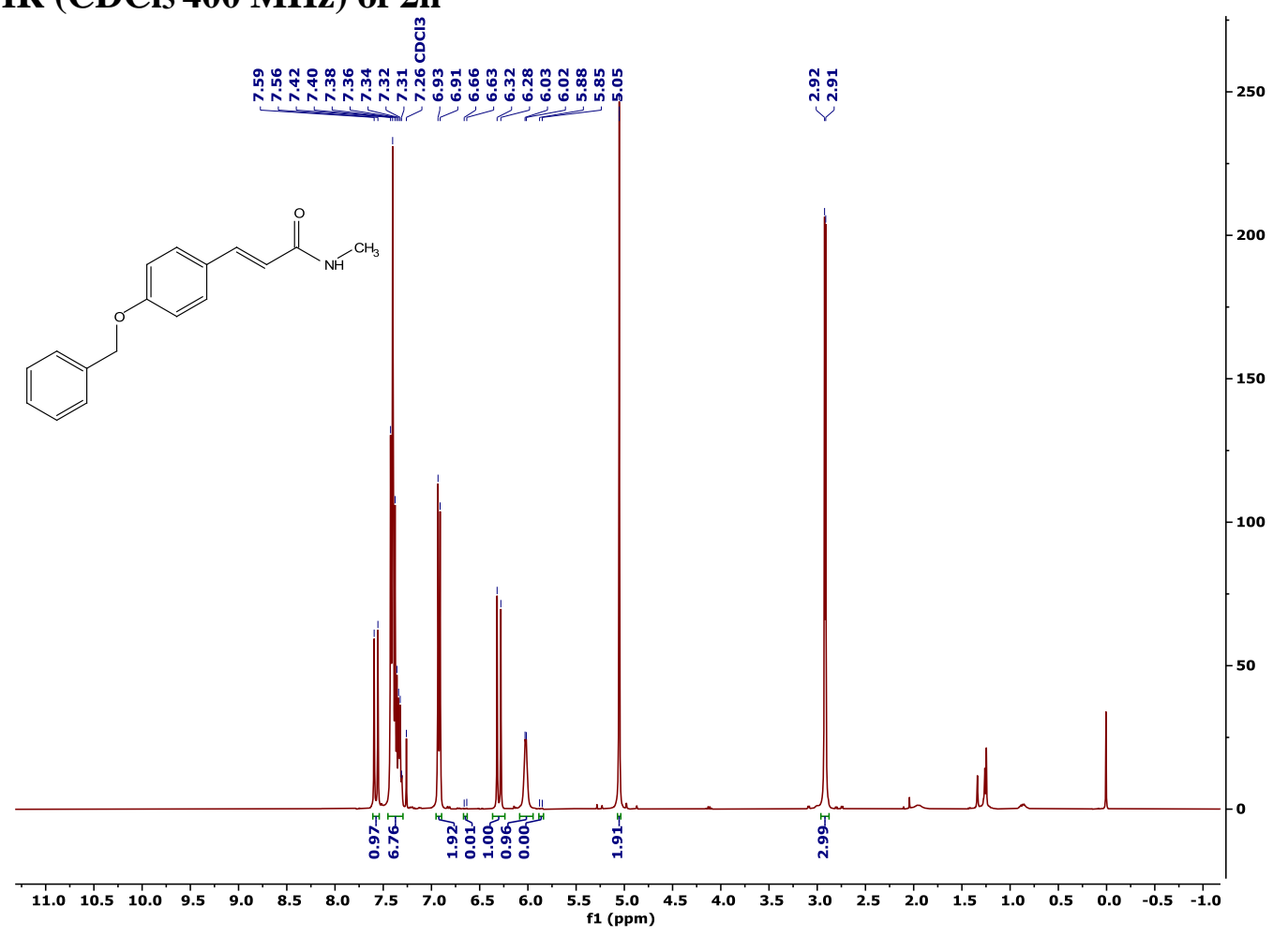

$\left.{ }^{13} \mathrm{C} \mathrm{NMR} \mathrm{(CDCl} 3,100 \mathrm{MHz}\right)$ of $2 \mathrm{~h}$

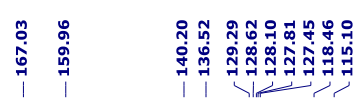

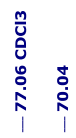
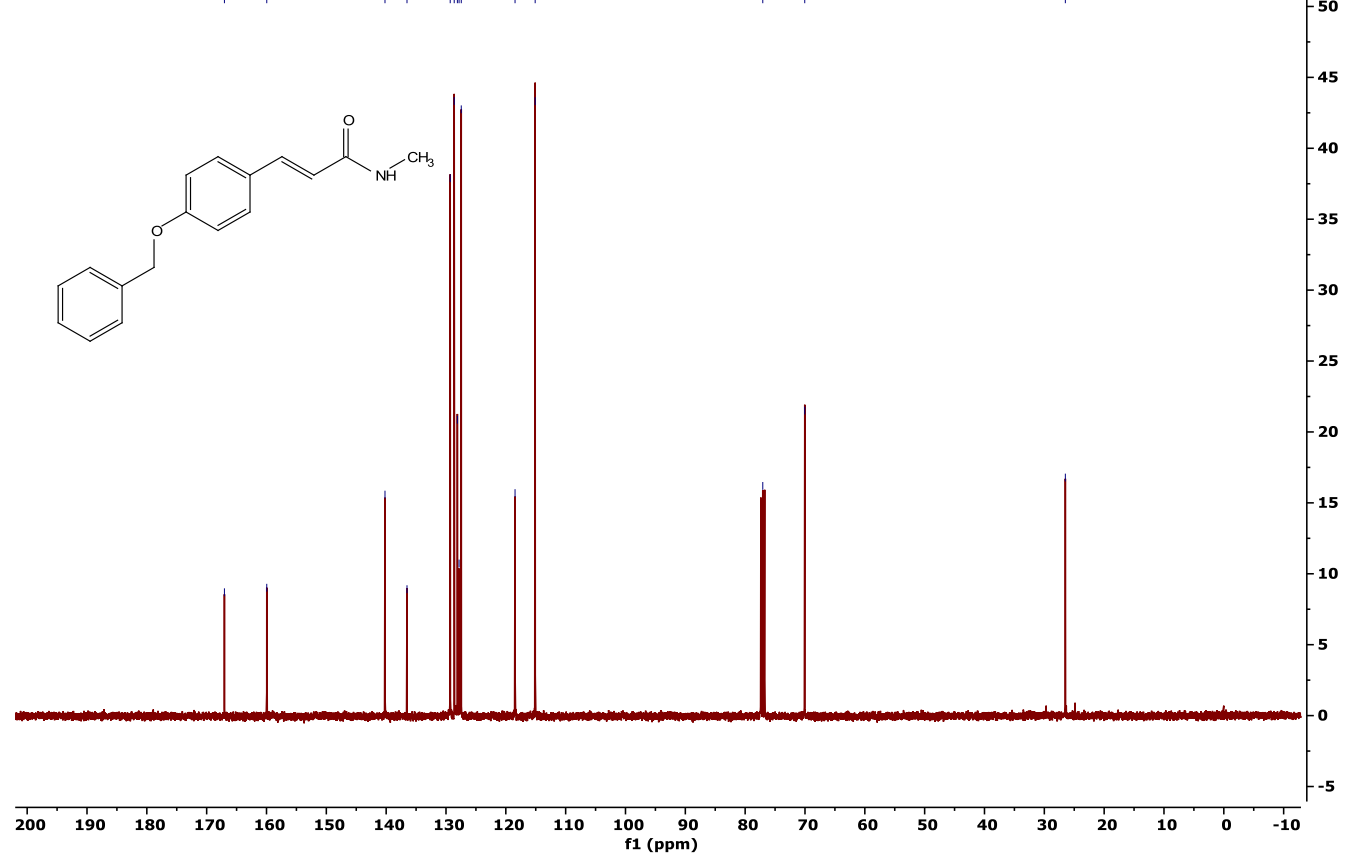
${ }^{1} \mathrm{H}$ NMR (CDCl $\left.400 \mathrm{MHz}\right)$ of $2 \mathrm{i}$

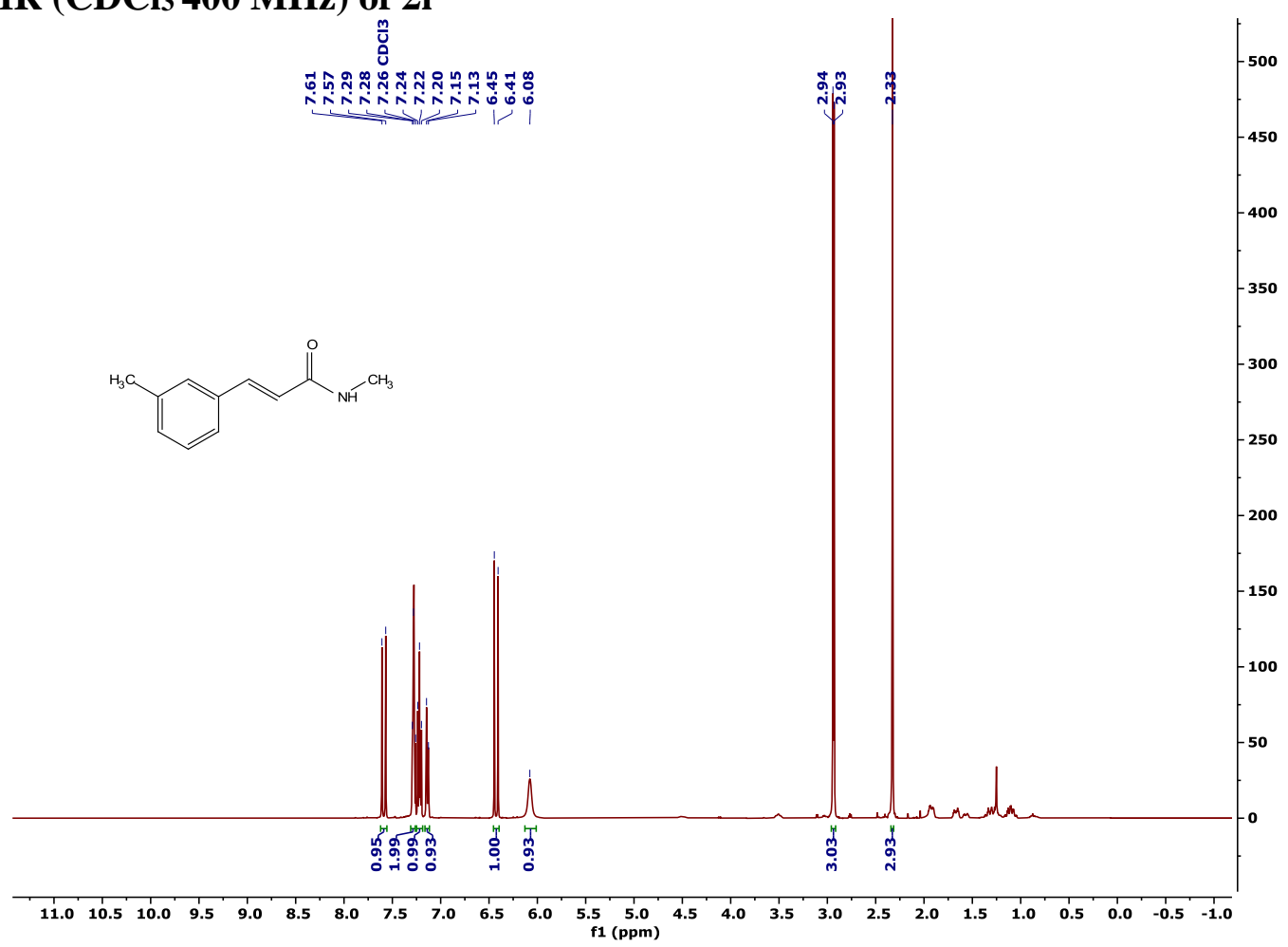

${ }^{13} \mathrm{C}$ NMR (CDCl $\left.3100 \mathrm{MHz}\right)$ of $2 \mathrm{i}$

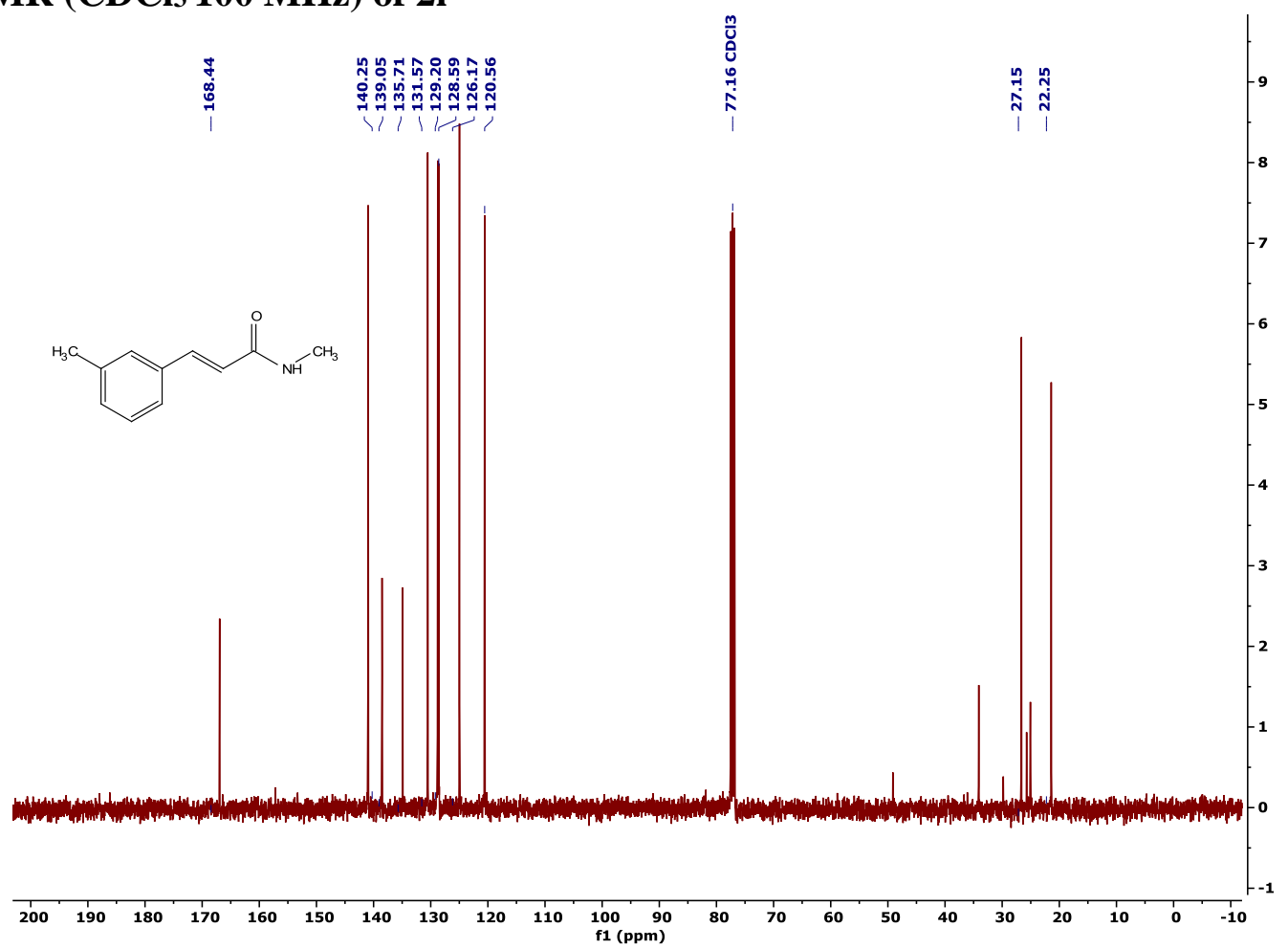


${ }^{1} \mathrm{H}$ NMR (CDCl $\left.400 \mathrm{MHz}\right)$ of $2 \mathrm{j}$

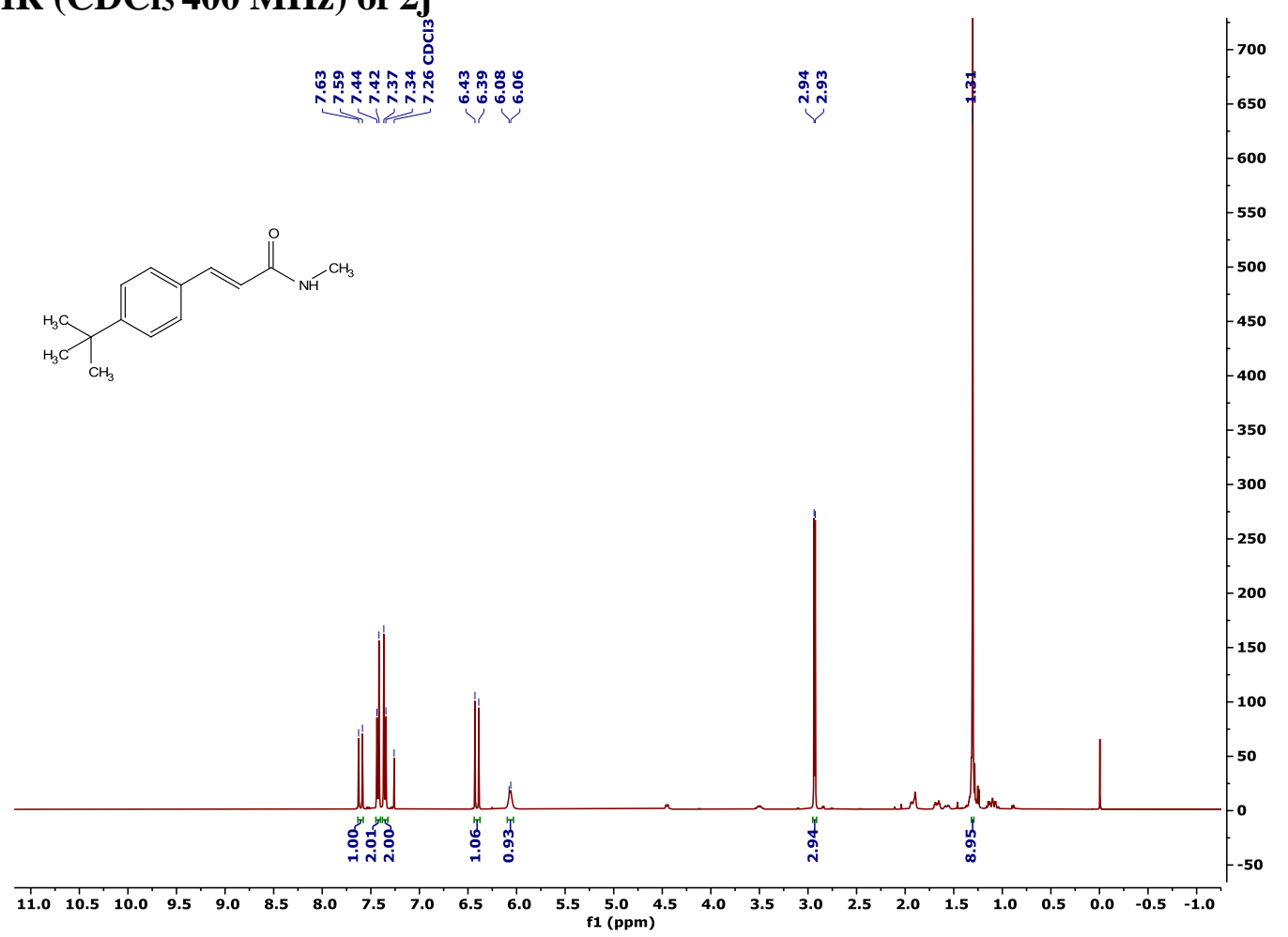

${ }^{13} \mathrm{C}$ NMR $\left(\mathrm{CDCl}_{3} 126 \mathrm{MHz}\right)$ of $2 \mathrm{j}$

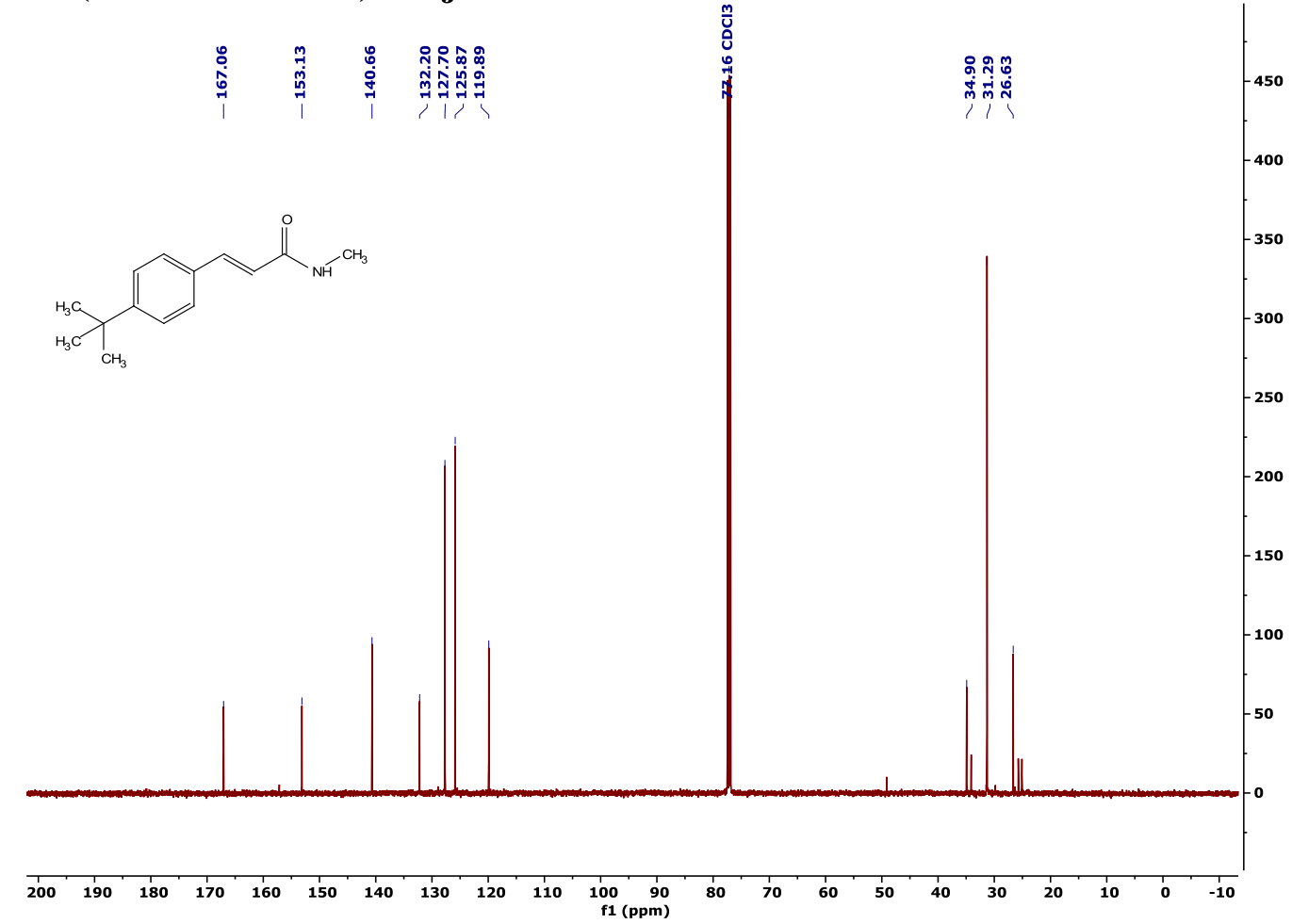


${ }^{1} \mathrm{H}$ NMR $\left(\mathrm{CDCl}_{3} 400 \mathrm{MHz}\right)$ of $2 \mathrm{k}$

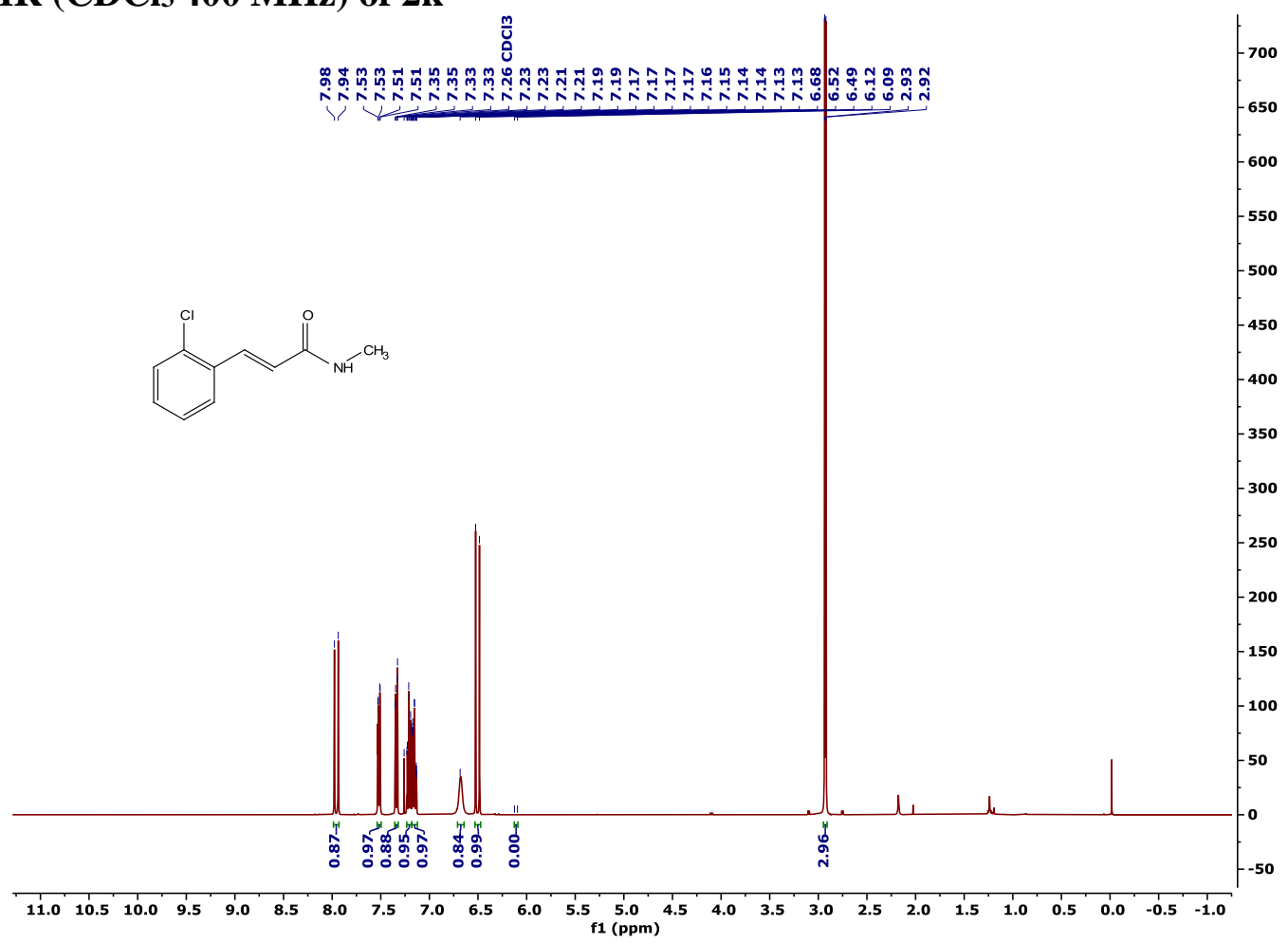

${ }^{13} \mathrm{C}$ NMR (CDCl3, $\left.100 \mathrm{MHz}\right)$ of $2 \mathrm{k}$

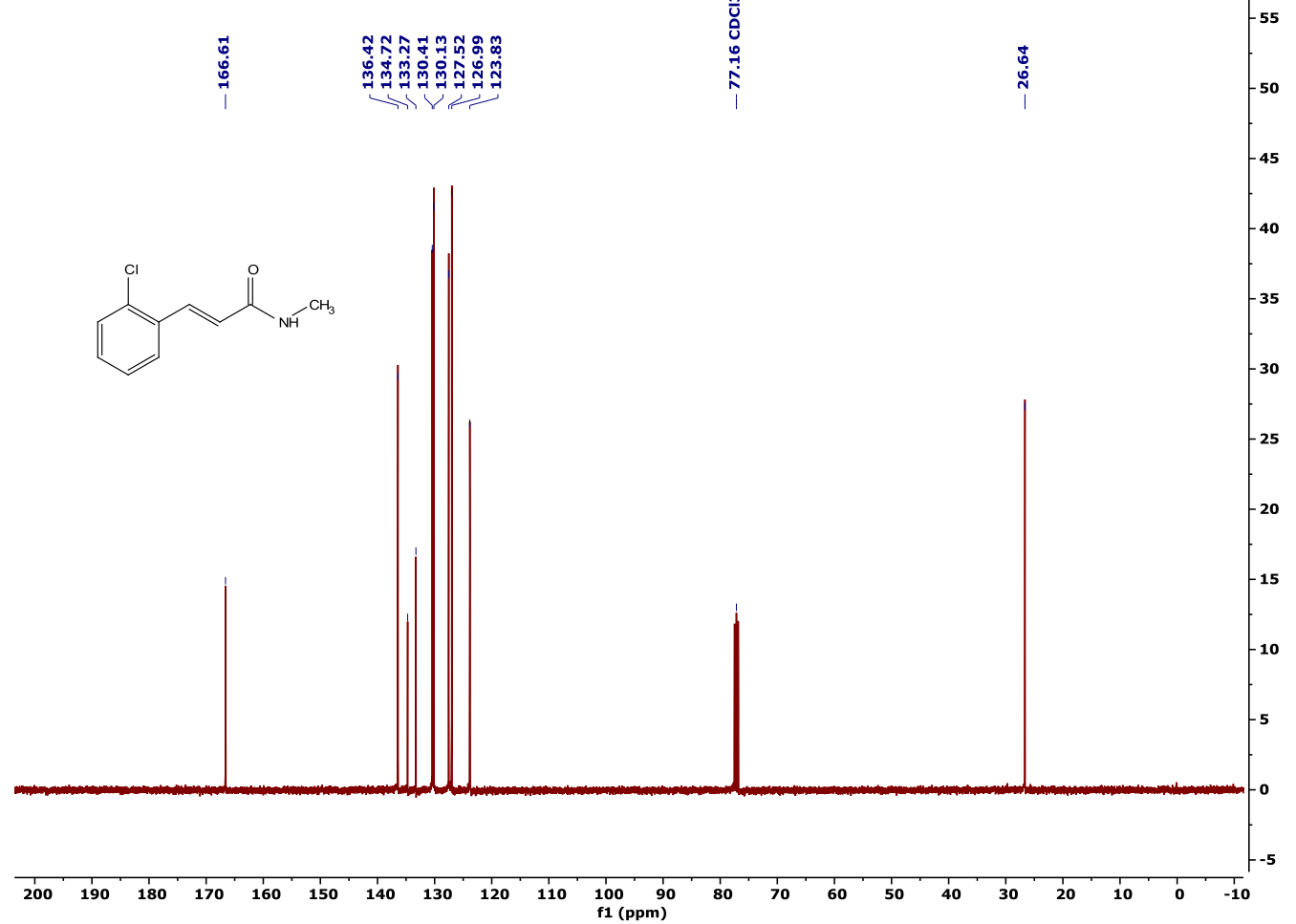


${ }^{1} \mathrm{H}$ NMR (Acetone-d $6400 \mathrm{MHz}$ ) of $2 \mathrm{l}$

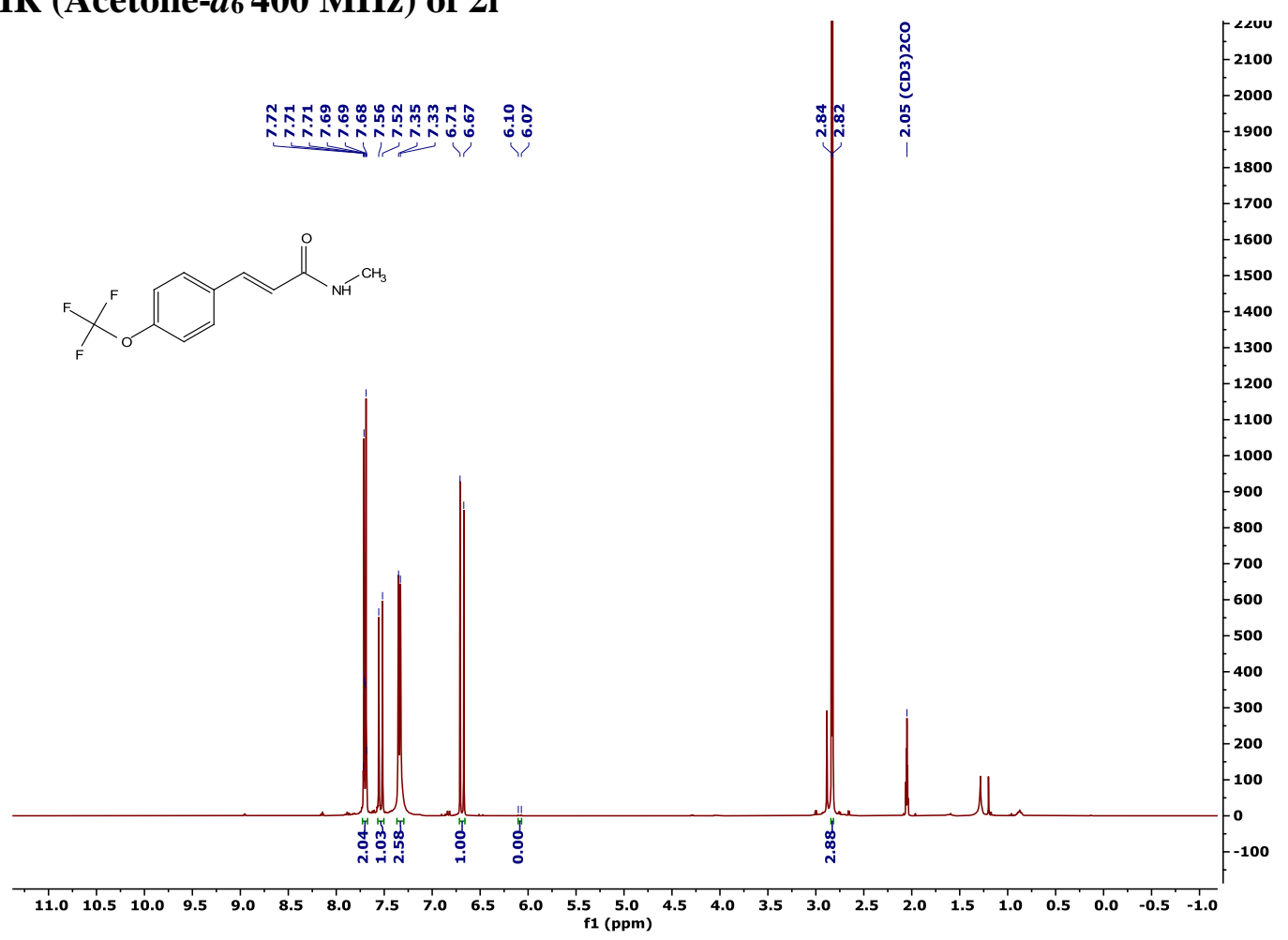

${ }^{19}$ F NMR (Acetone- $d_{6} 376 \mathrm{MHz}$ ) of $2 \mathrm{I}$

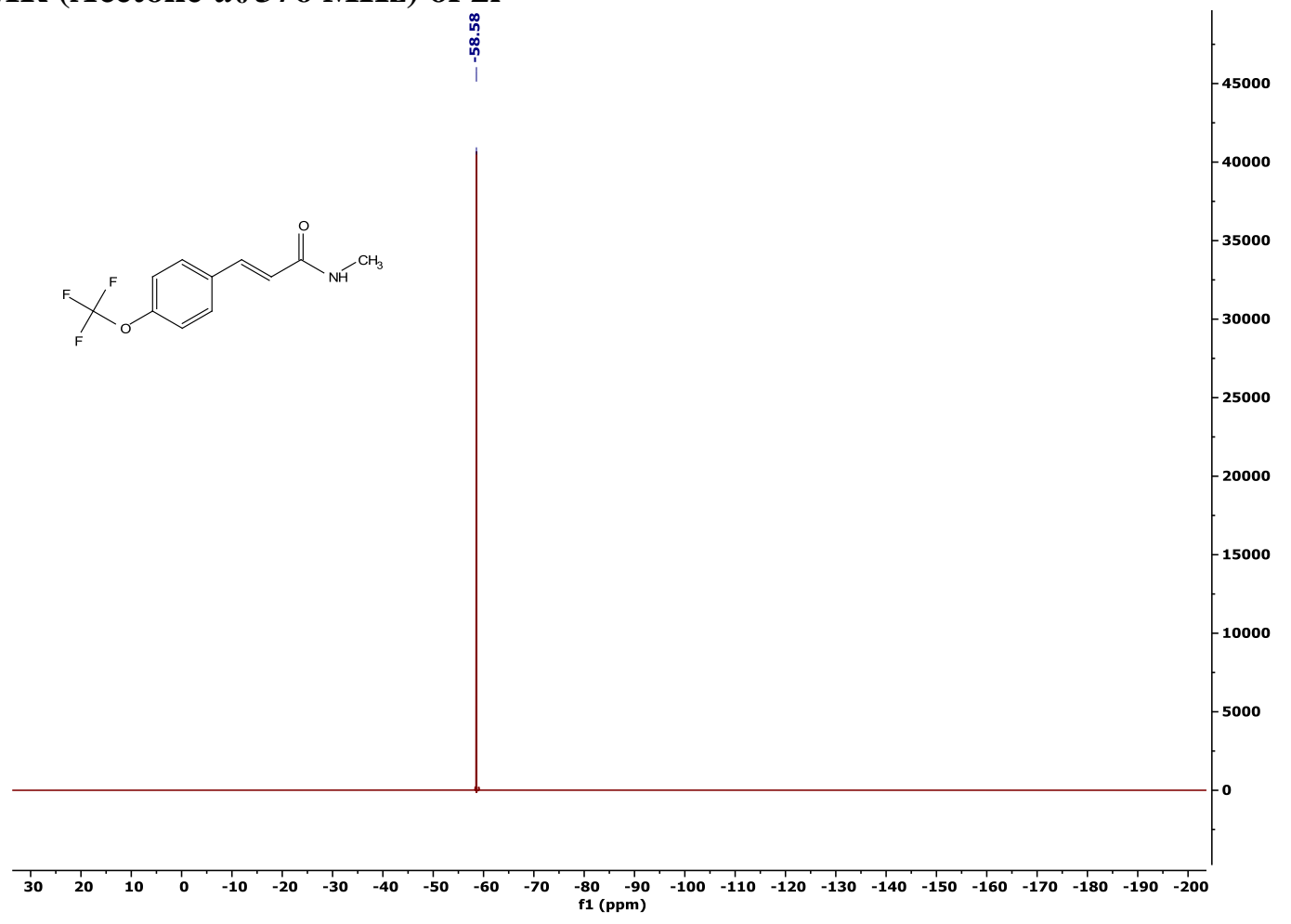


${ }^{13} \mathrm{C}$ NMR (Acetone-d $d_{6} 100 \mathrm{MHz}$ ) of $2 \mathrm{I}$

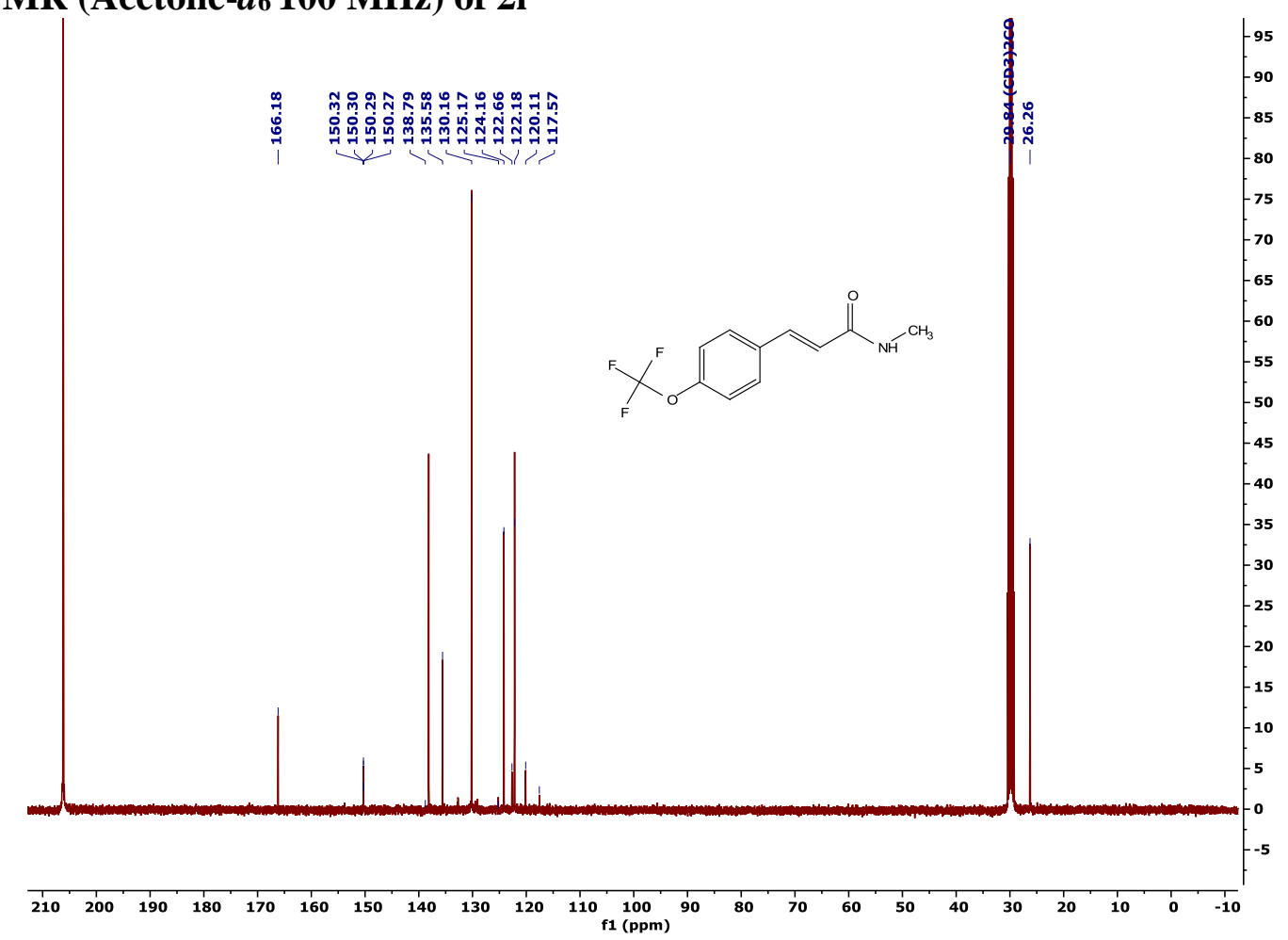

${ }^{1} \mathrm{H} \mathrm{NMR}\left(\mathrm{CDCl}_{3}, 400 \mathrm{MHz}\right)$ of $2 \mathrm{~m}$

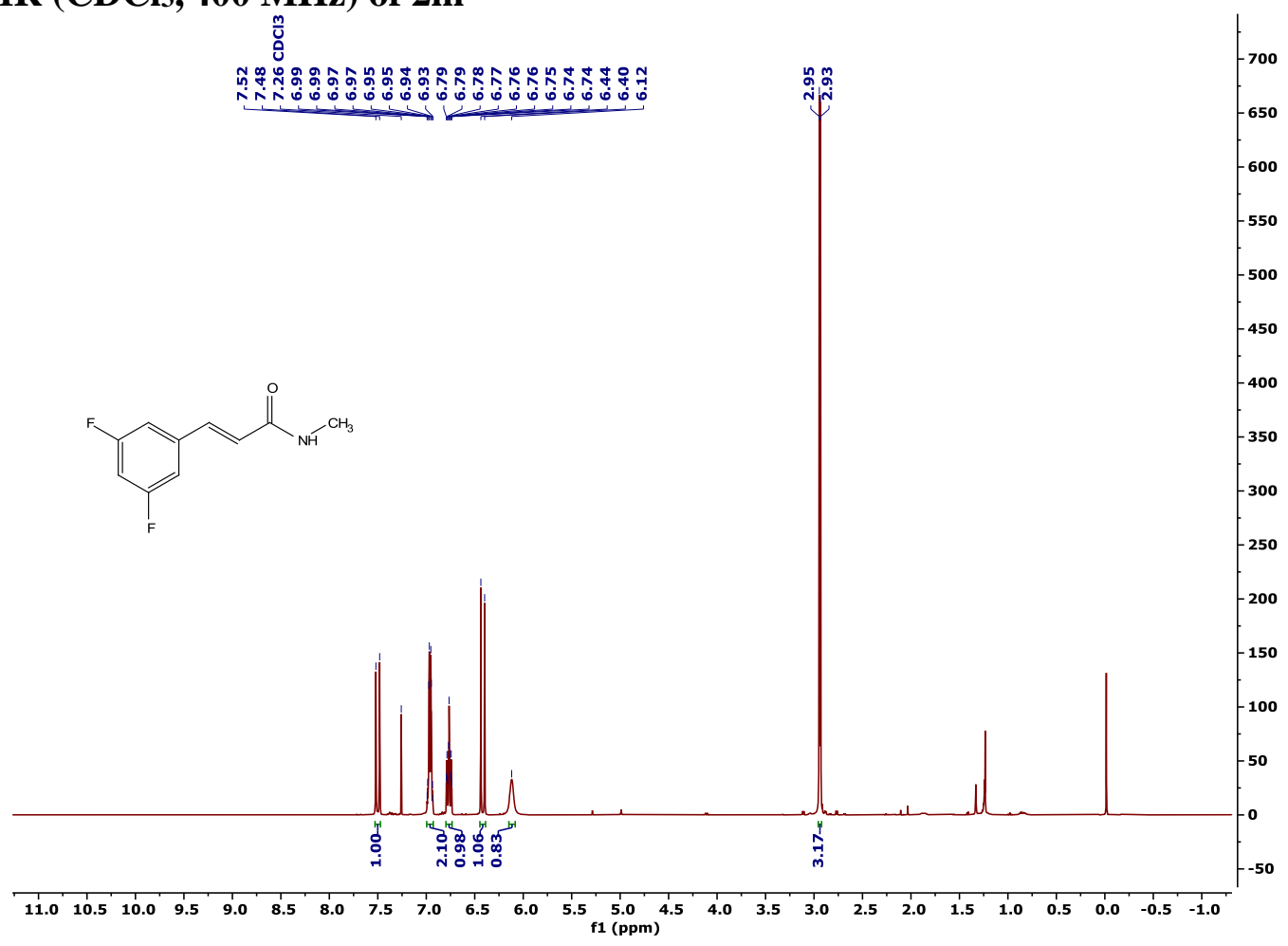


${ }^{19} \mathrm{~F}$ NMR $\left(\mathrm{CDCl}_{3} 376 \mathrm{MHz}\right)$ of $2 \mathrm{~m}$

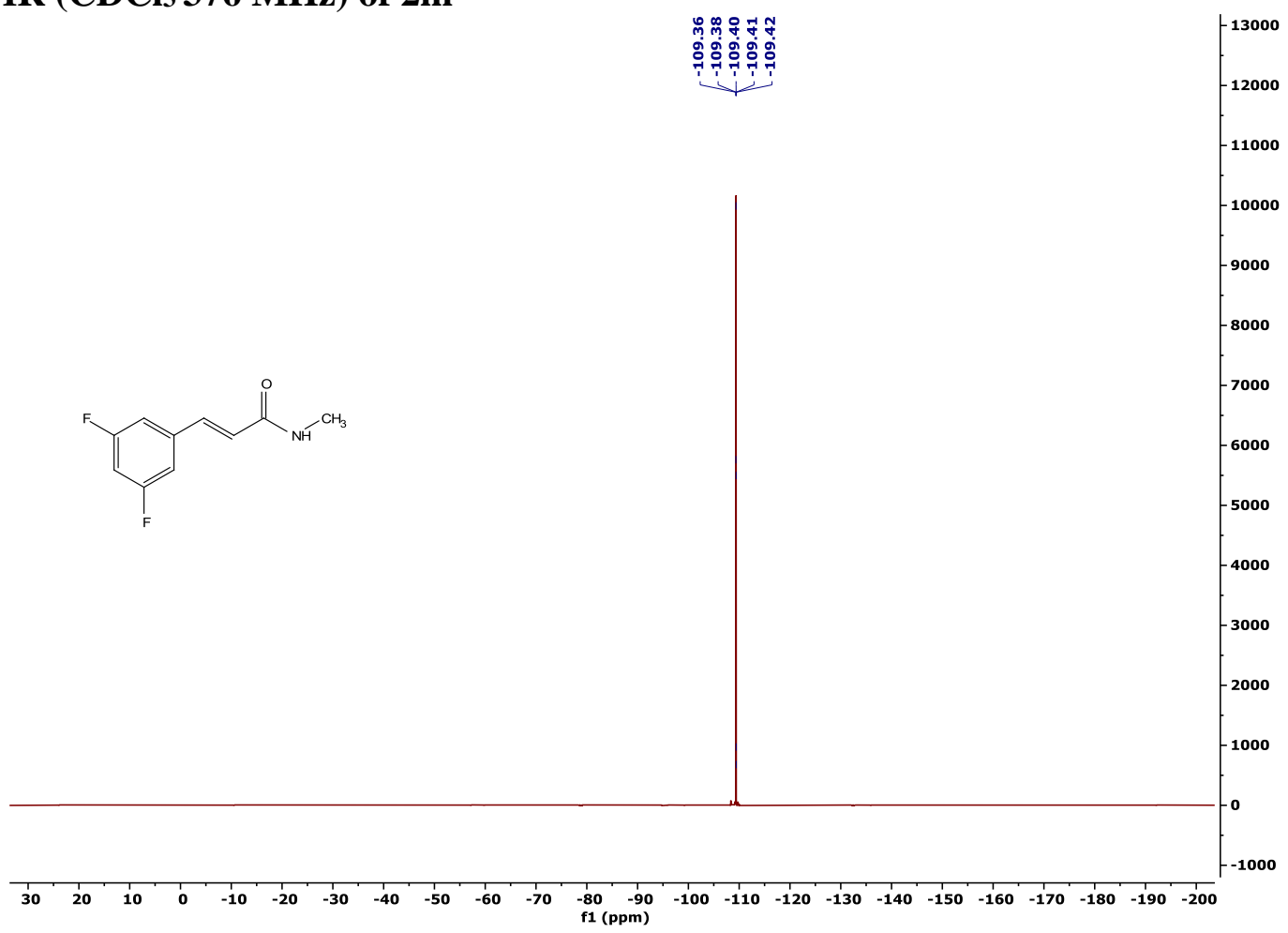

${ }^{13} \mathrm{C} \mathrm{NMR}\left(\mathrm{CDCl}_{3} 100 \mathrm{MHz}\right)$ of $2 \mathrm{~m}$

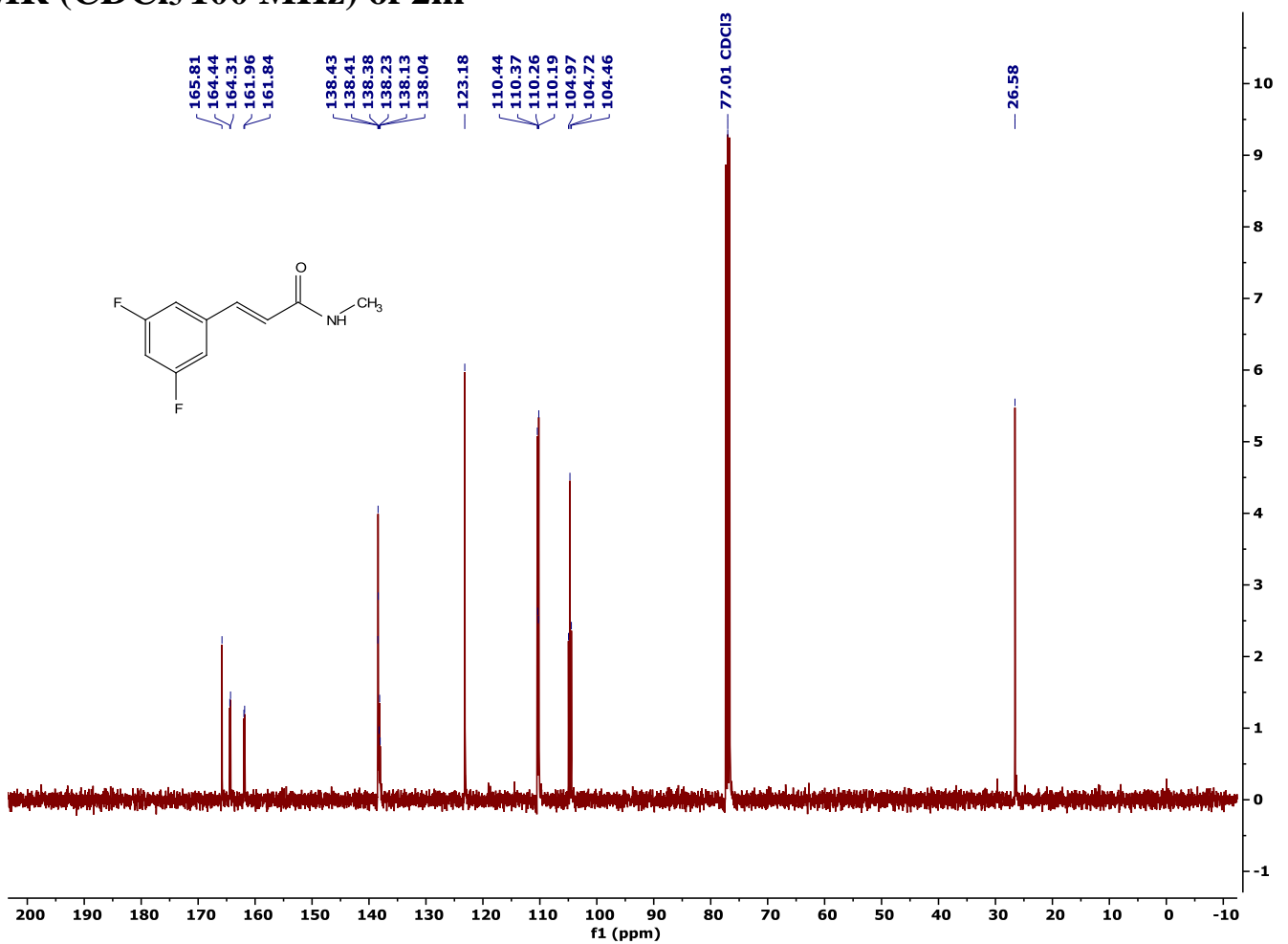


${ }^{1} \mathrm{H}$ NMR (CD $30 D 400 \mathrm{MHz}$ ) of $2 \mathrm{n}$

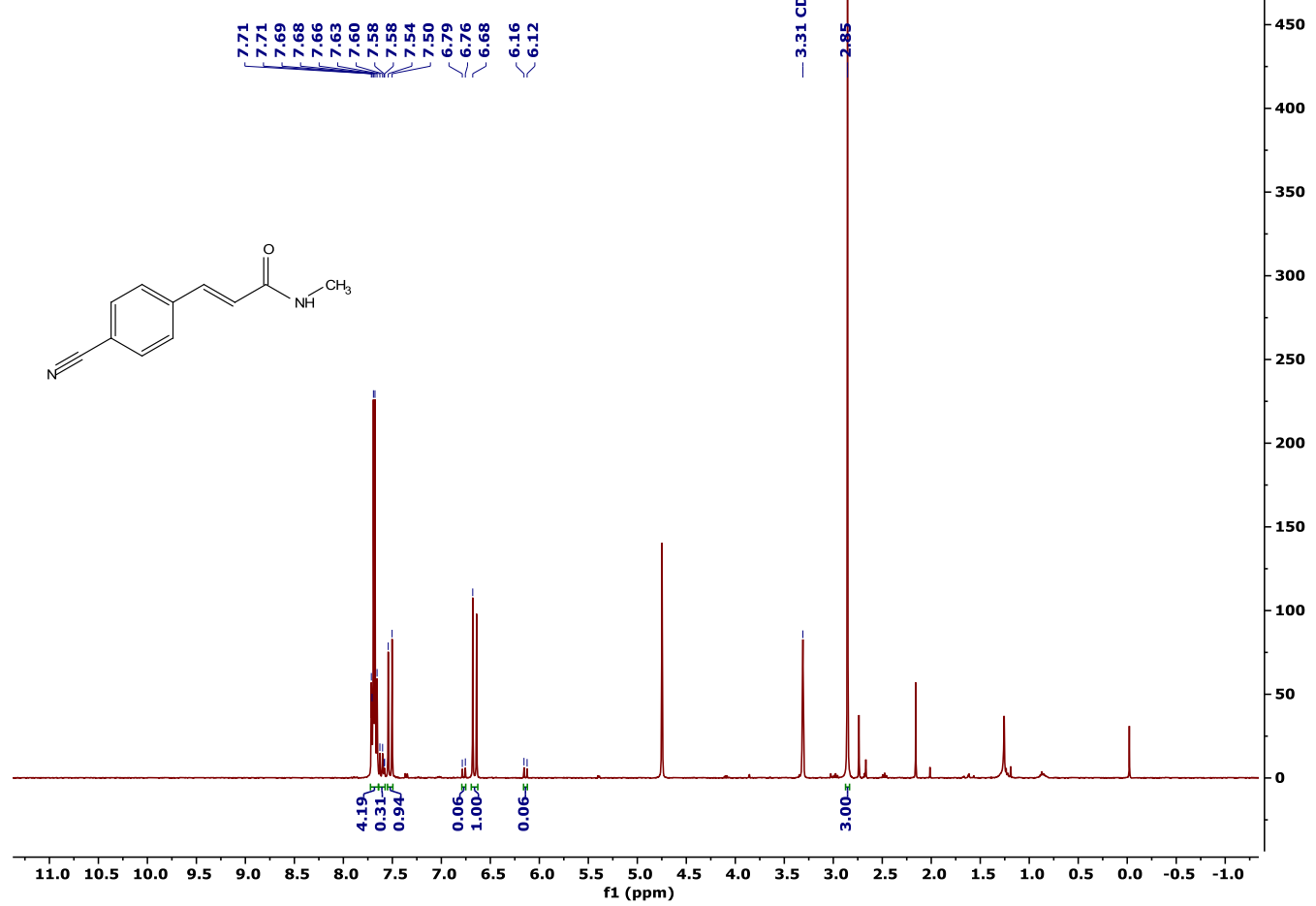

${ }^{13} \mathrm{C}$ NMR (CD3OD $100 \mathrm{MHz}$ ) of $2 \mathrm{n}$

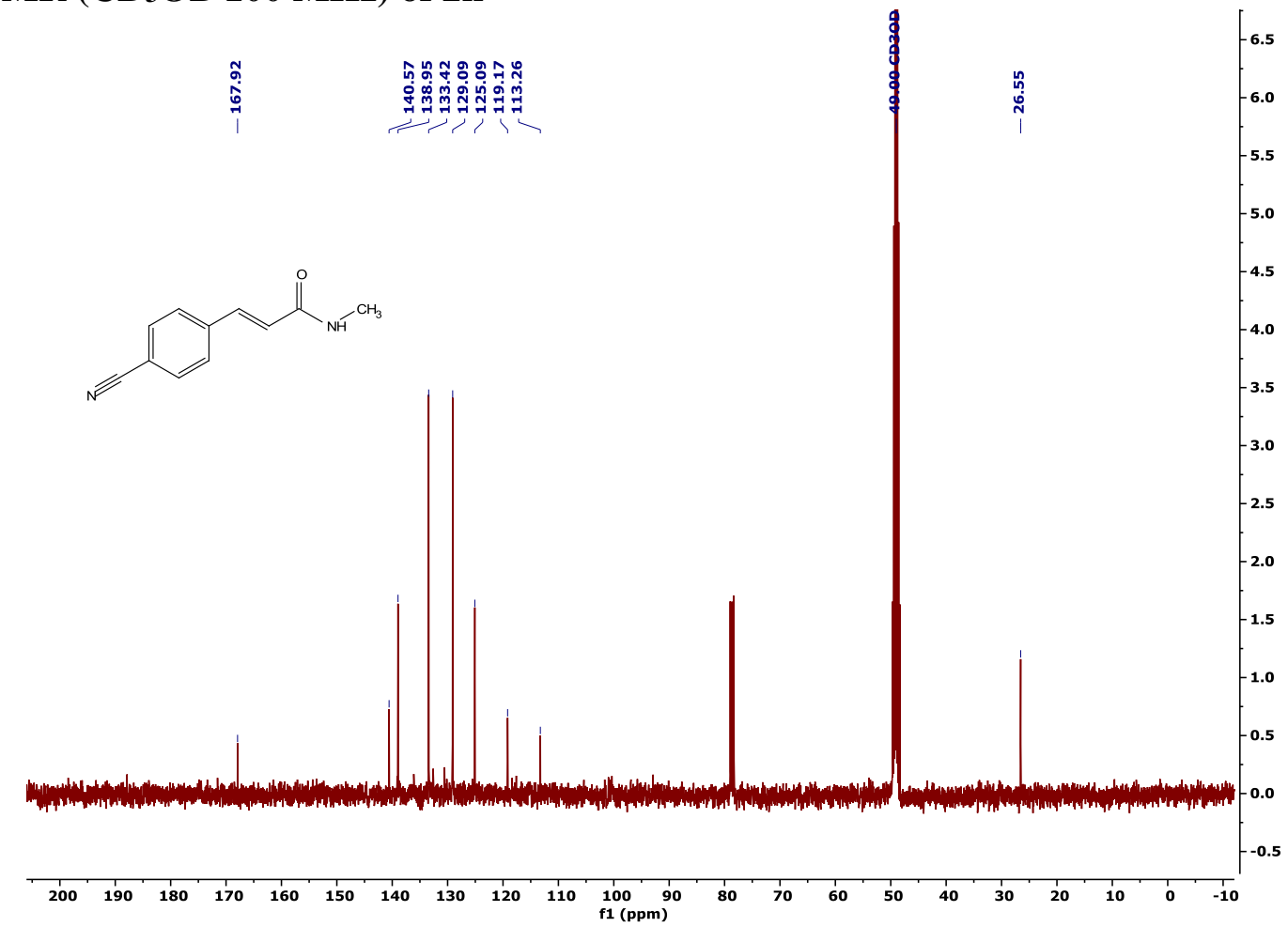


${ }^{1} \mathrm{H}$ NMR $\left(\mathrm{CDCl}_{3} 400 \mathrm{MHz}\right)$ of 20

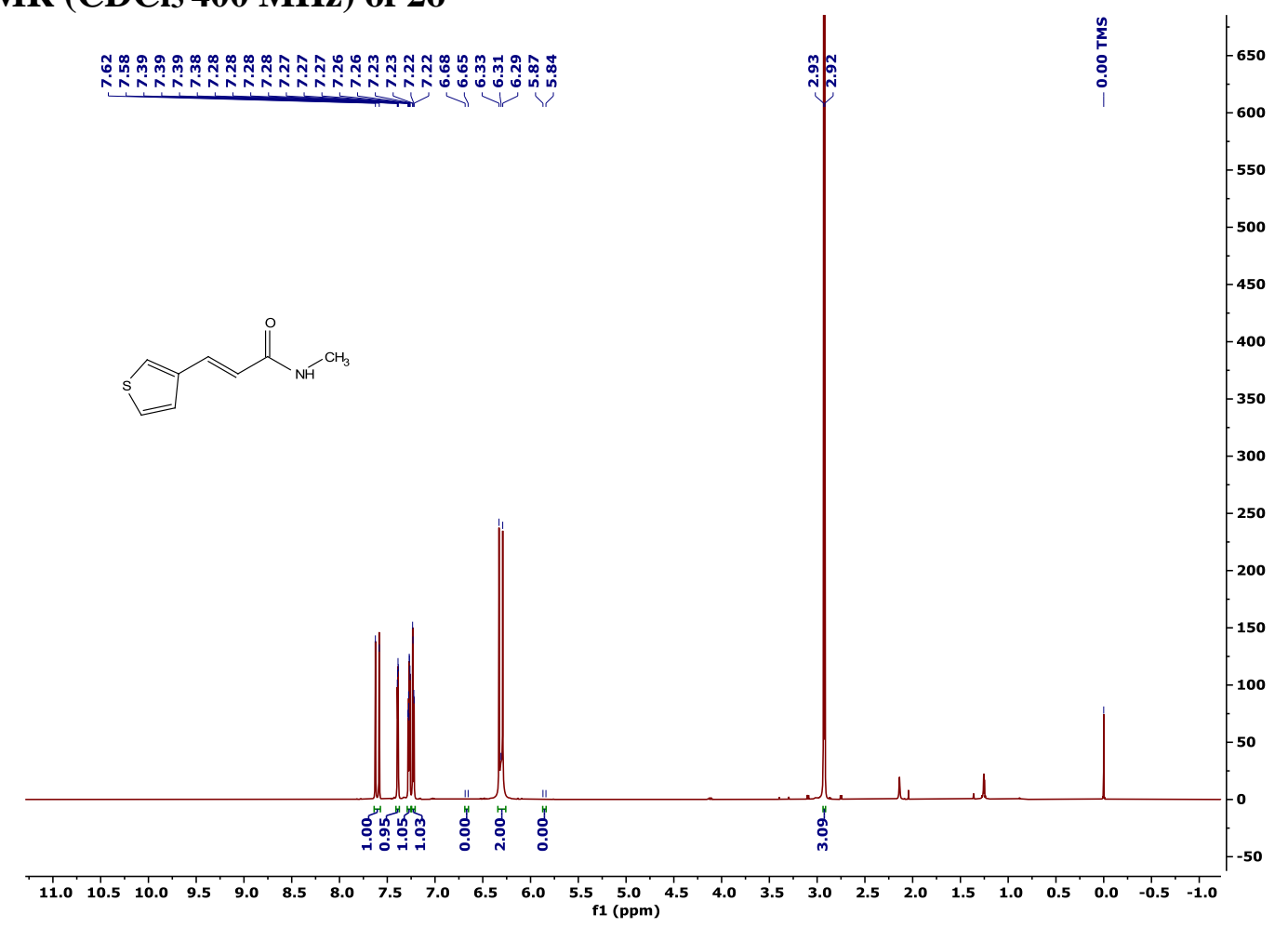

${ }^{13} \mathrm{C} \mathrm{NMR}\left(\mathrm{CDCl}_{3} 100 \mathrm{MHz}\right)$ of 20

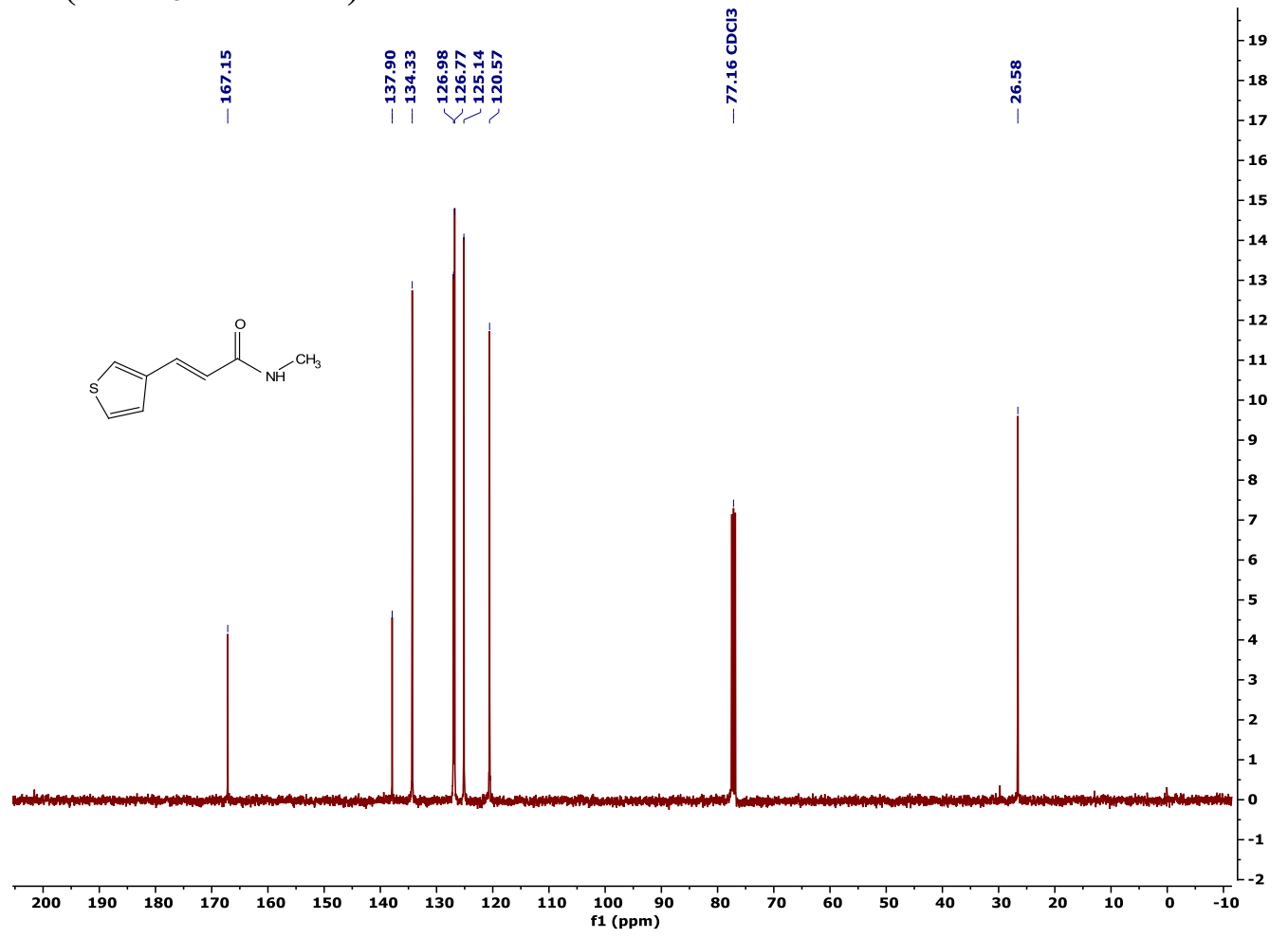


${ }^{1} \mathrm{H}$ NMR (CD $\left.{ }_{3} \mathrm{OD} 400 \mathrm{MHz}\right)$ of $2 \mathrm{p}$

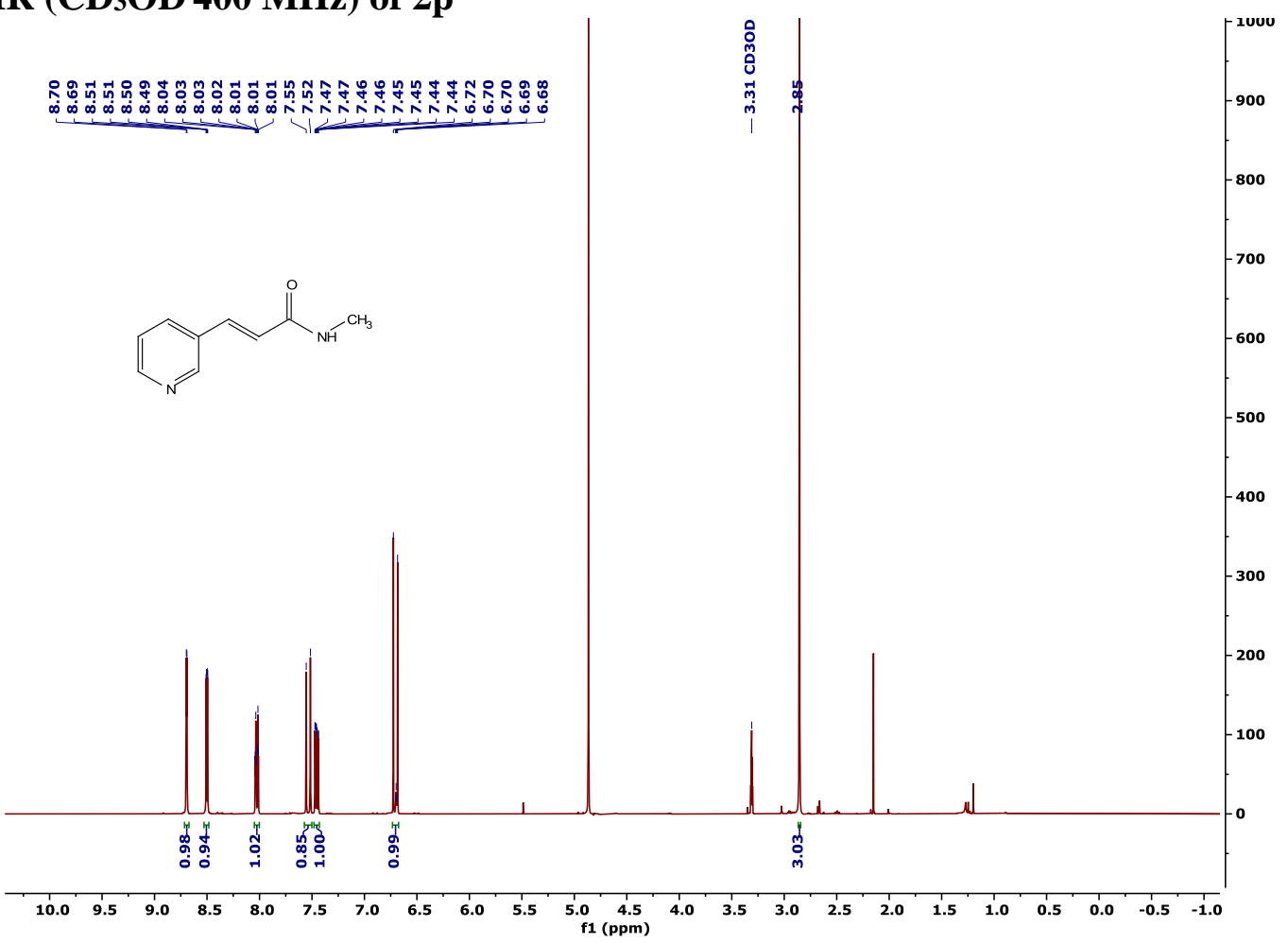

${ }^{13} \mathrm{C}$ NMR (CD ${ }_{3} \mathrm{OD} 100 \mathrm{MHz}$ ) of $2 \mathrm{p}$

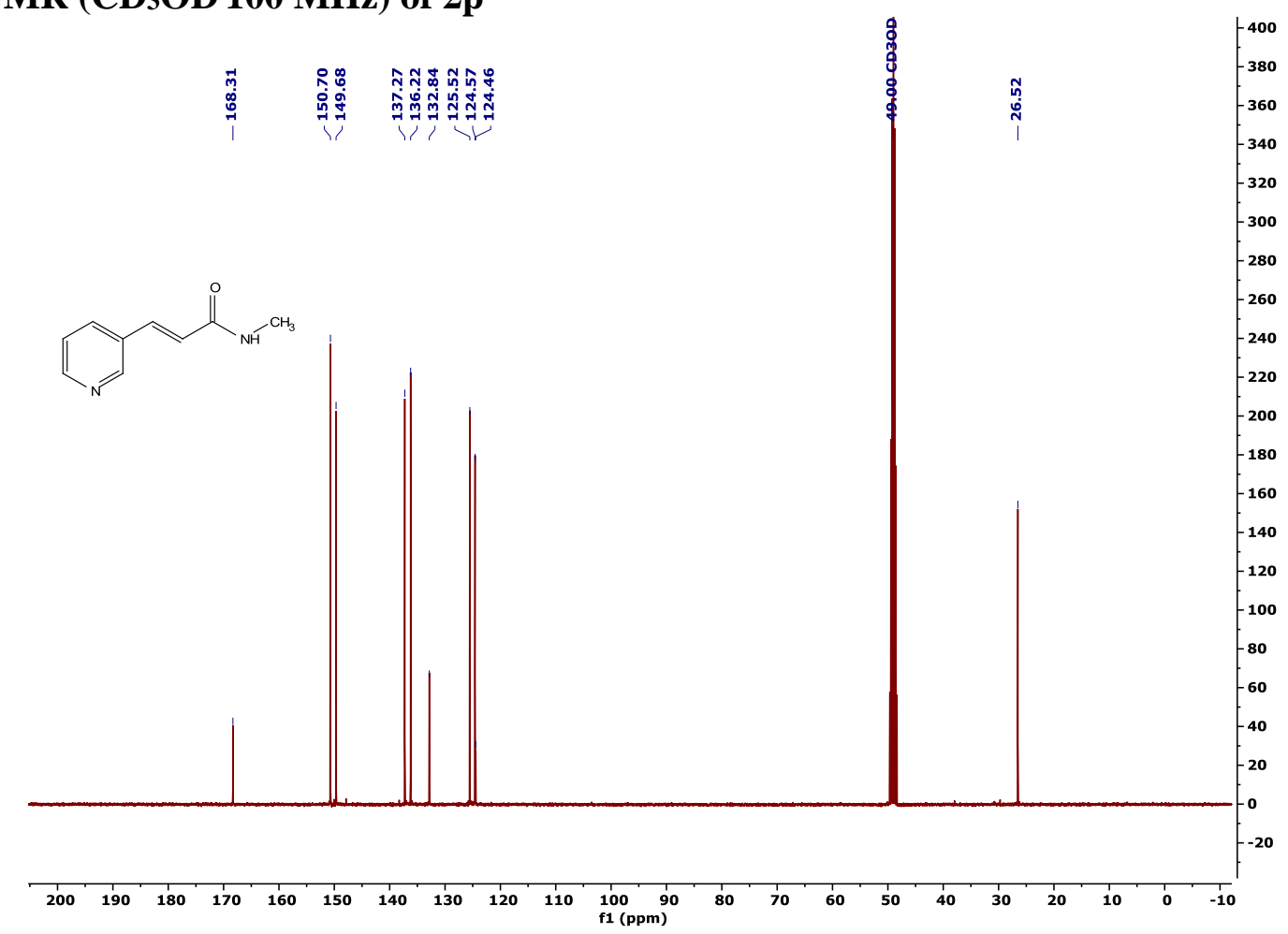


${ }^{1} \mathrm{H}$ NMR $\left(\mathrm{CDCl}_{3} 400 \mathrm{MHz}\right)$ of $4 \mathrm{a}$

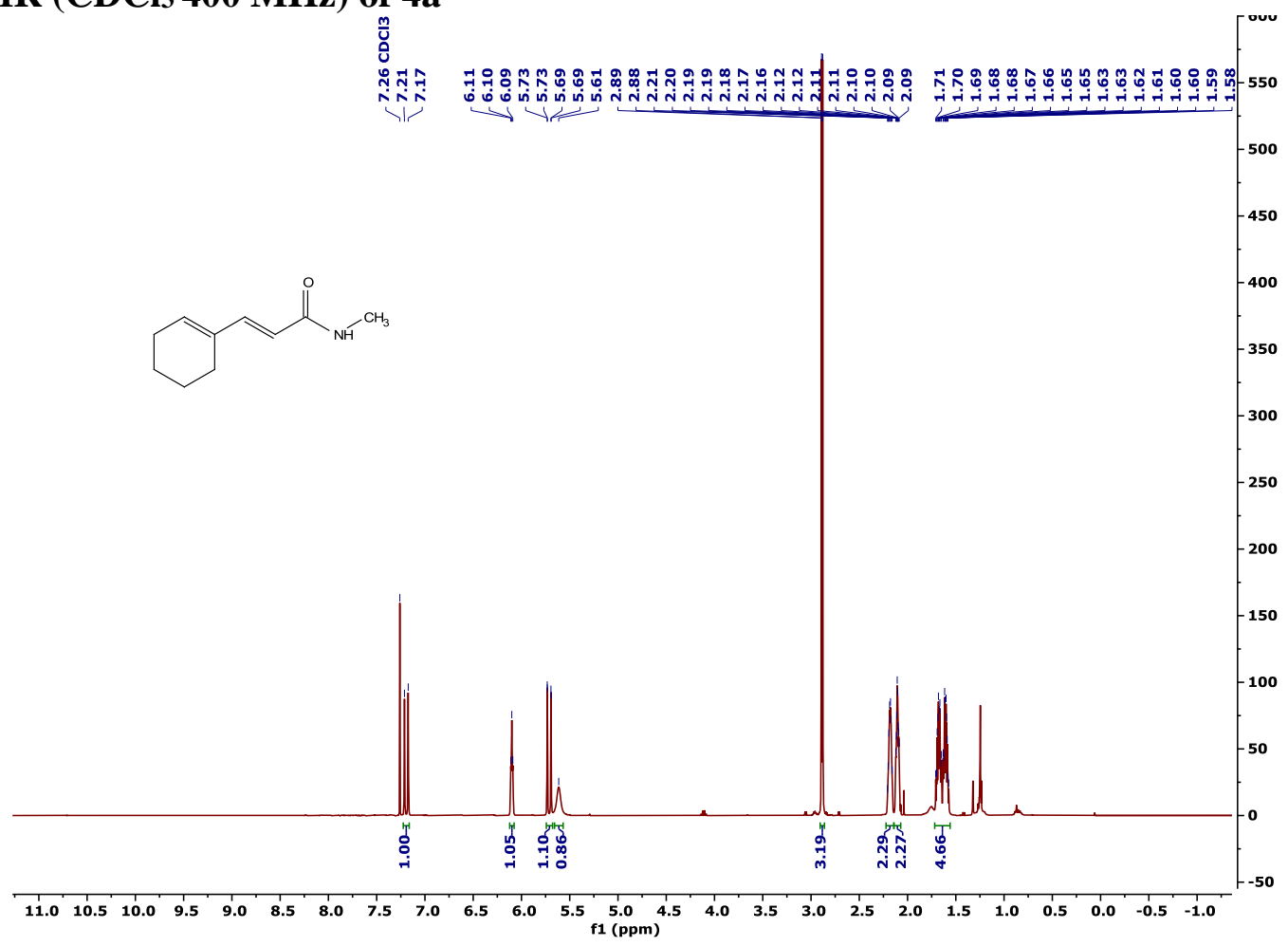

${ }^{13} \mathrm{C}$ NMR $\left(\mathrm{CDCl}_{3} 100 \mathrm{MHz}\right)$ of $4 \mathrm{a}$

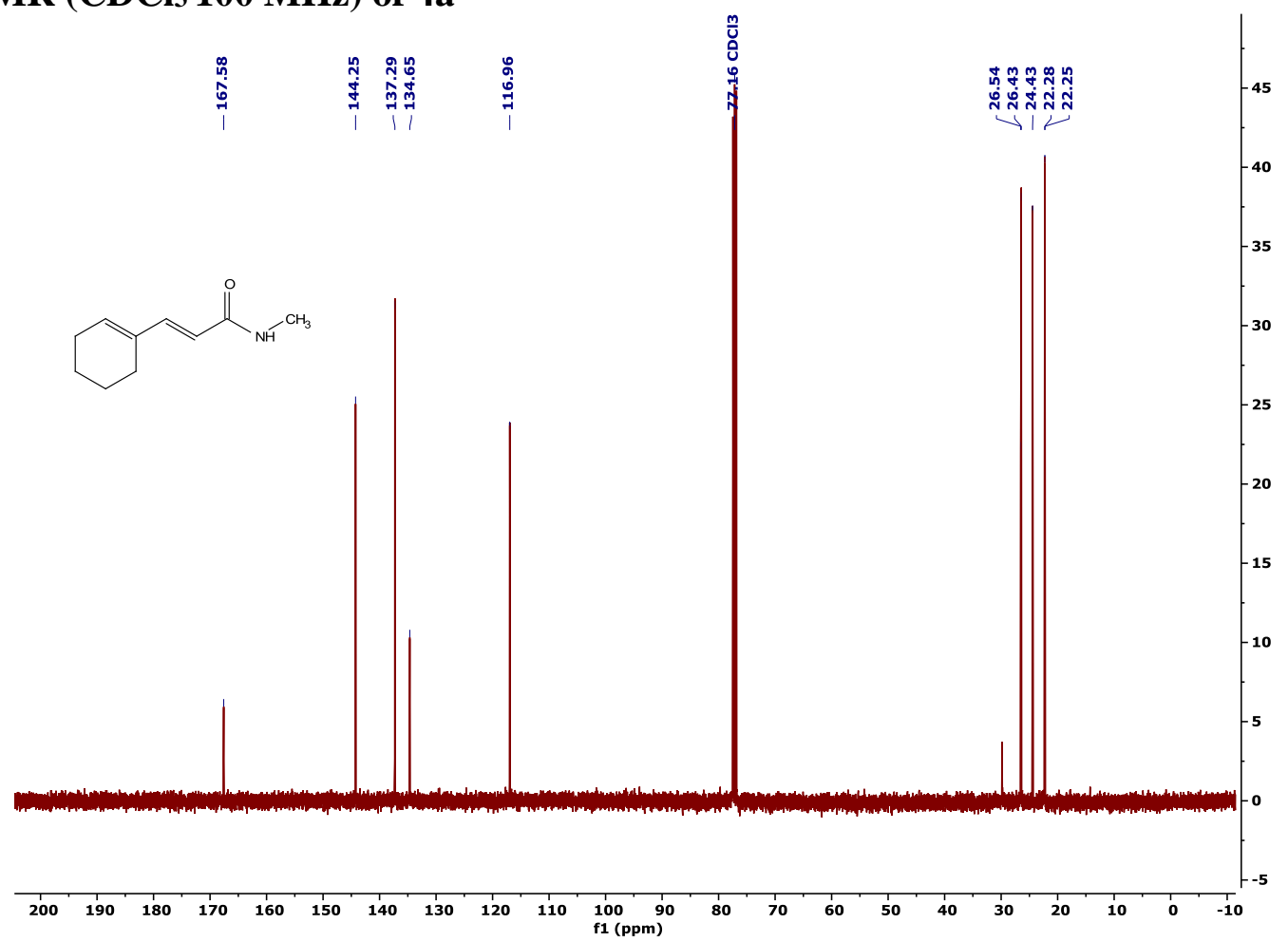


${ }^{1} \mathrm{H}$ NMR $\left(\mathrm{CDCl}_{3} 400 \mathrm{MHz}\right)$ of $4 \mathrm{~b}$

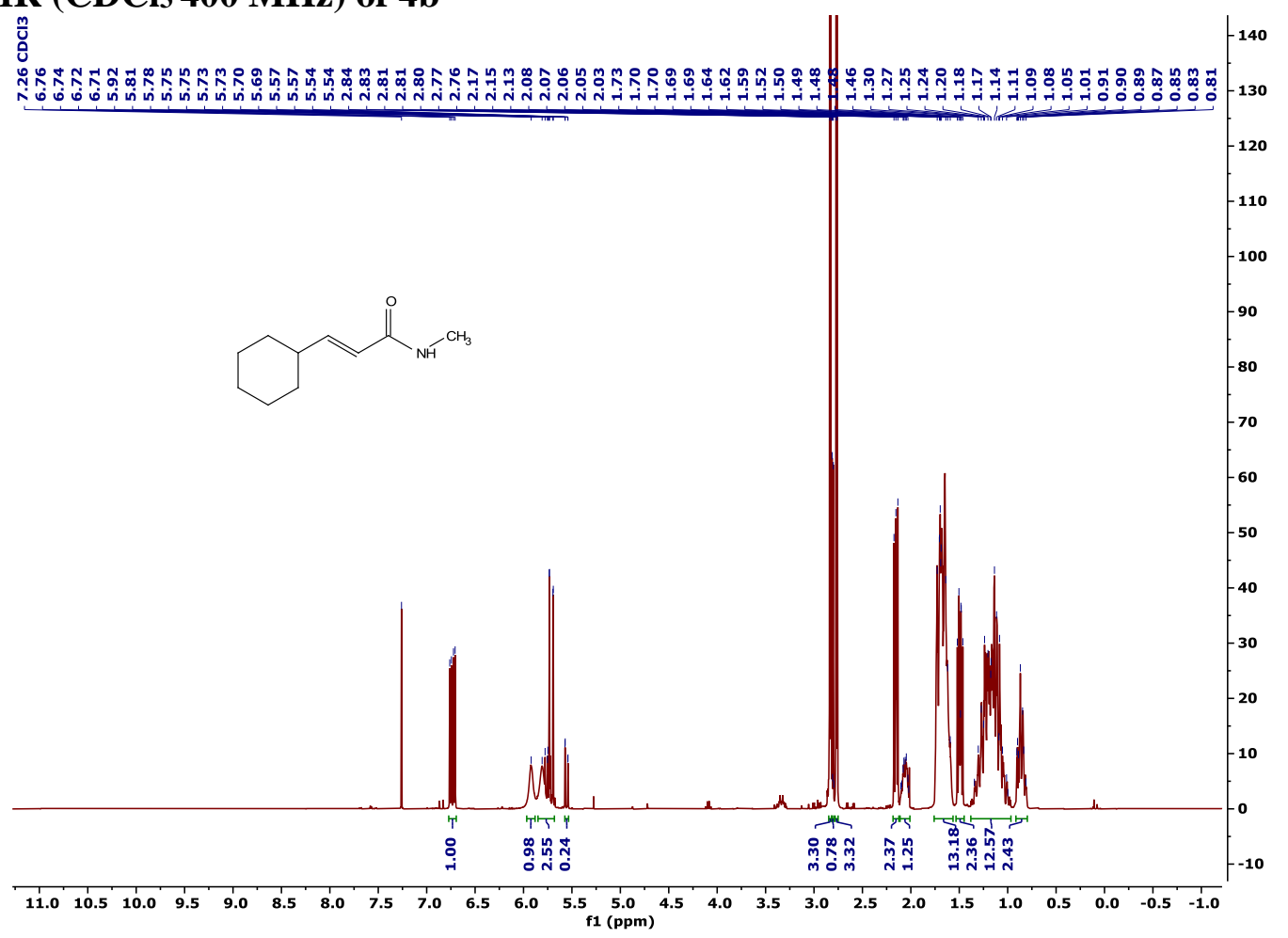

${ }^{13} \mathrm{C}$ NMR ( $\left.\mathrm{CDCl}_{3} 100 \mathrm{MHz}\right)$ of $4 \mathrm{~b}$

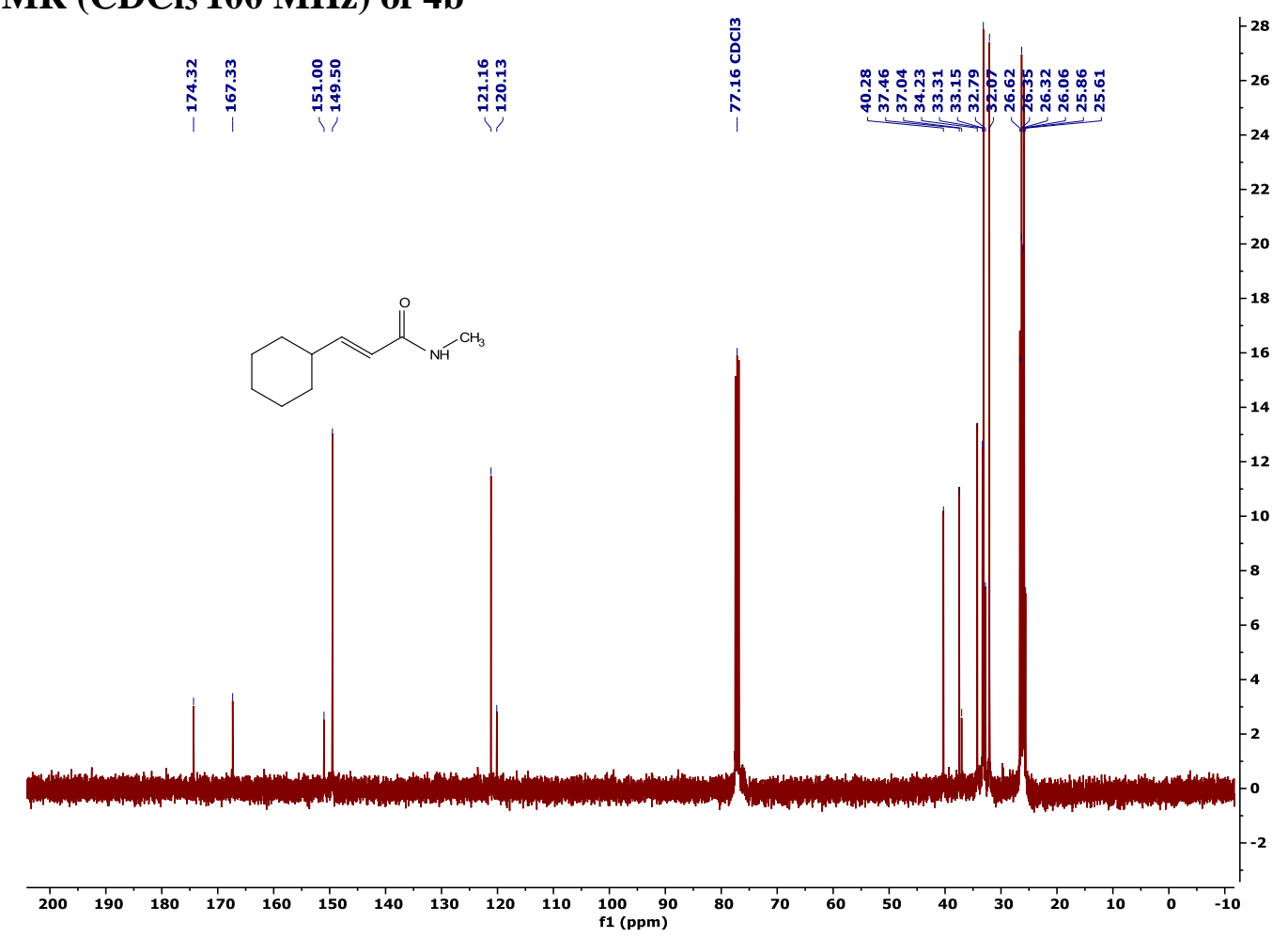


${ }^{1} \mathrm{H}$ NMR ( $\left.\mathrm{CDCl}_{3} 400 \mathrm{MHz}\right)$ of $4 \mathrm{c}$

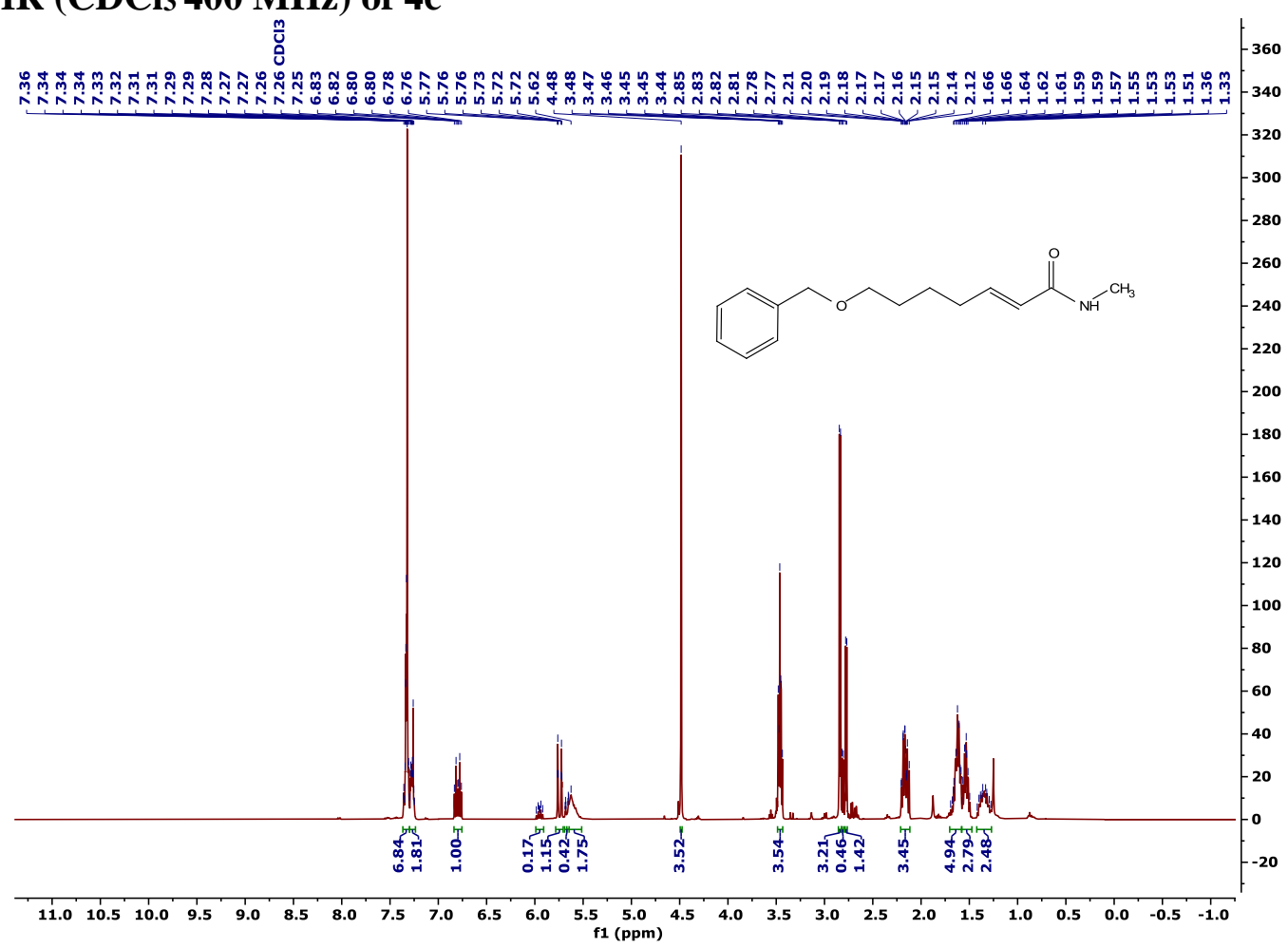

${ }^{13} \mathrm{C}$ NMR (CDCl $\left.3100 \mathrm{MHz}\right)$ of $4 \mathrm{c}$

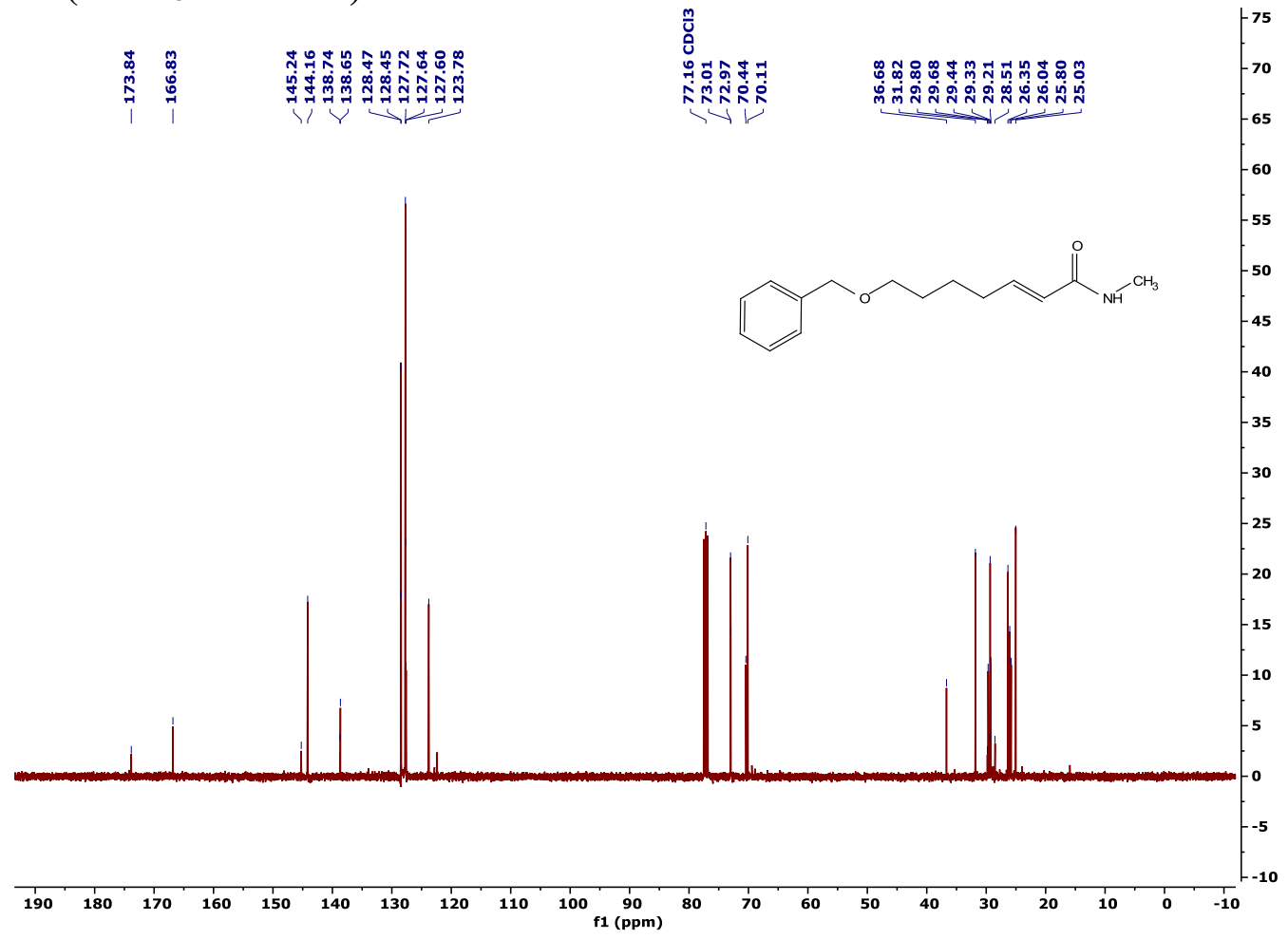


${ }^{1} \mathrm{H}$ NMR $\left(\mathrm{CDCl}_{3} 400 \mathrm{MHz}\right)$ of $4 d$

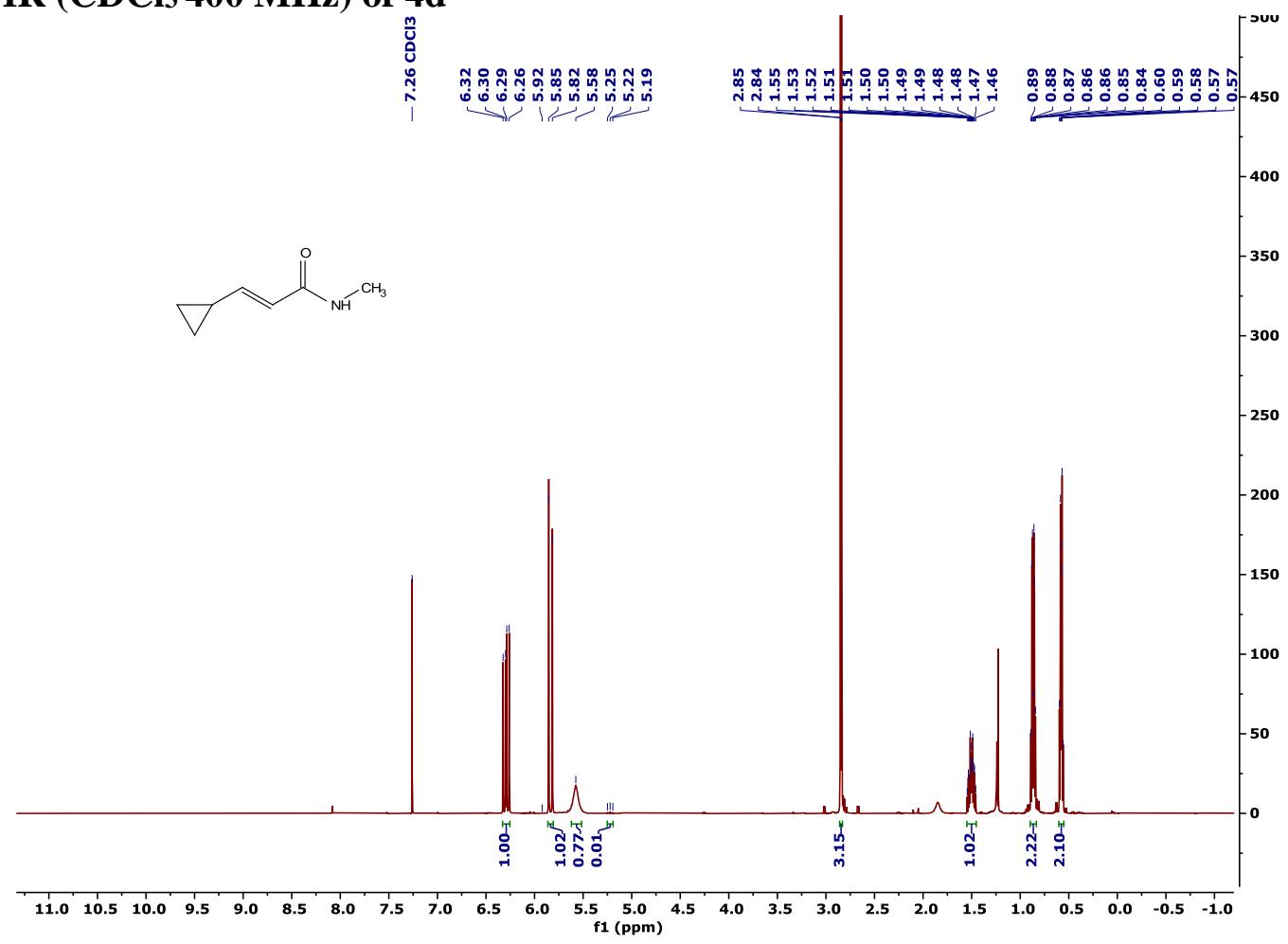

${ }^{13} \mathrm{C} \mathrm{NMR}\left(\mathrm{CDCl}_{3} 100 \mathrm{MHz}\right)$ of $4 \mathrm{~d}$

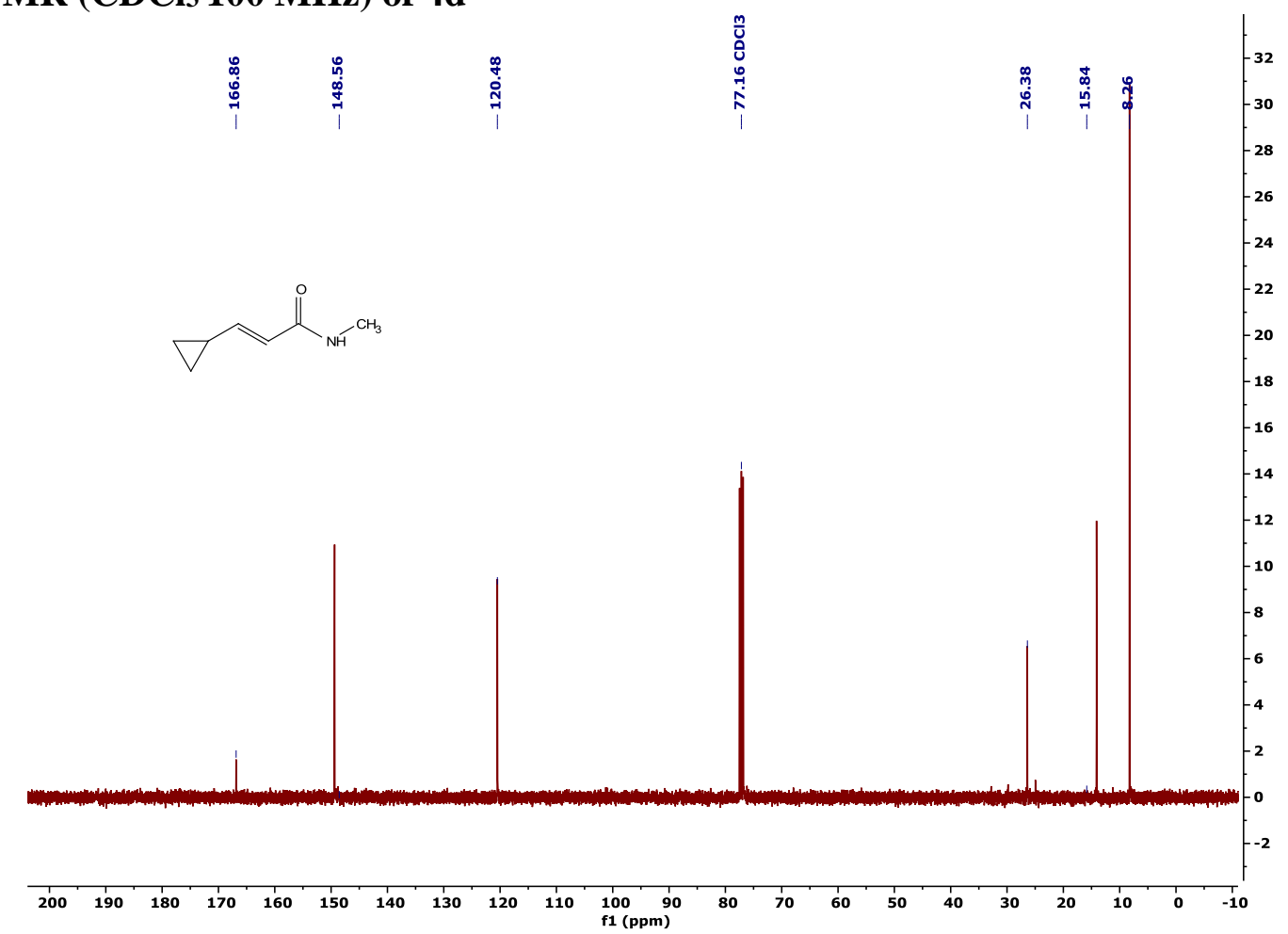


${ }^{1} \mathrm{H}$ NMR $\left(\mathrm{CDCl}_{3} 400 \mathrm{MHz}\right)$ of $4 \mathrm{e}$

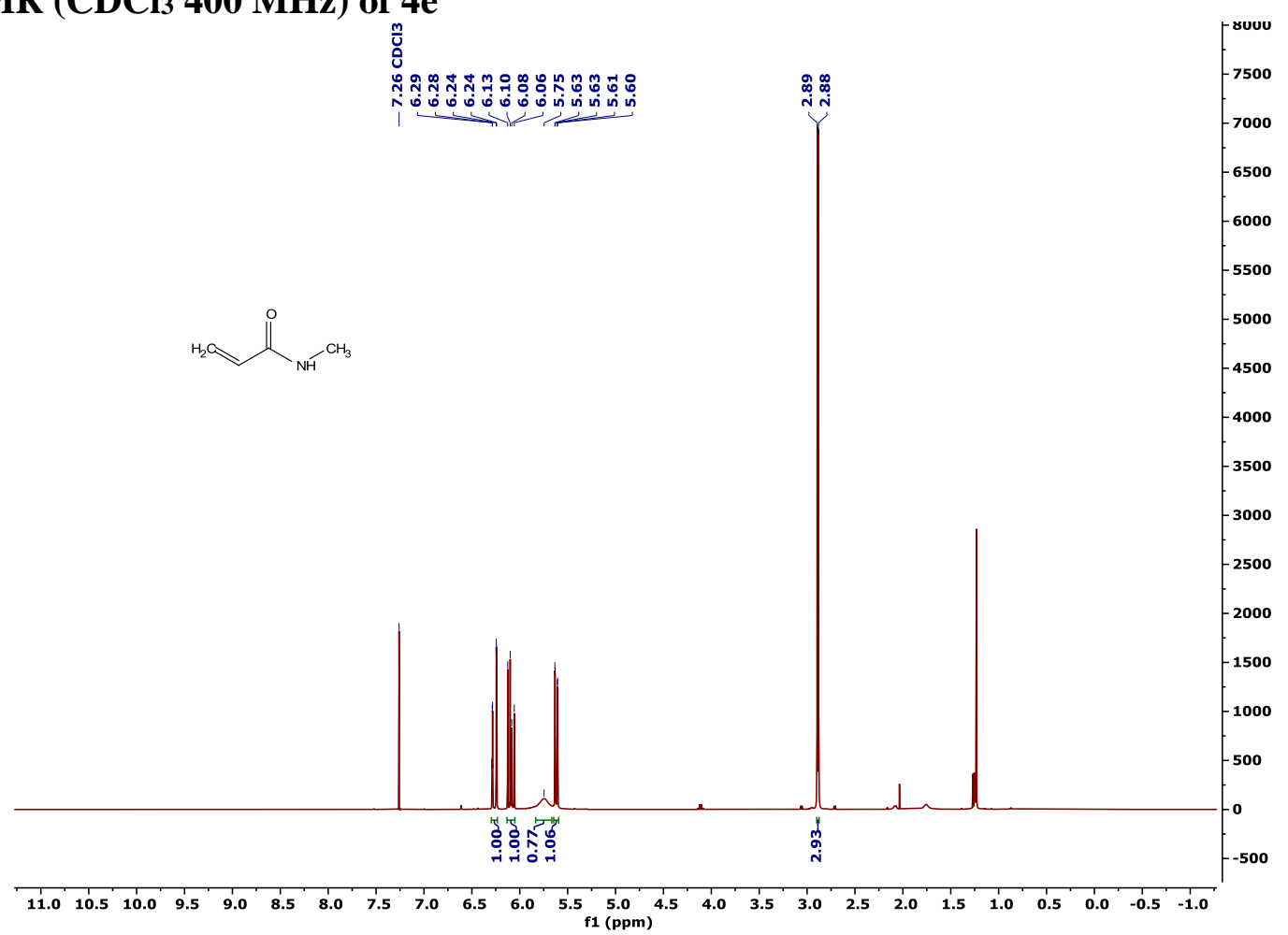

${ }^{13} \mathrm{C}$ NMR ( $\left.\mathrm{CDCl}_{3} 100 \mathrm{MHz}\right)$ of $4 \mathrm{e}$

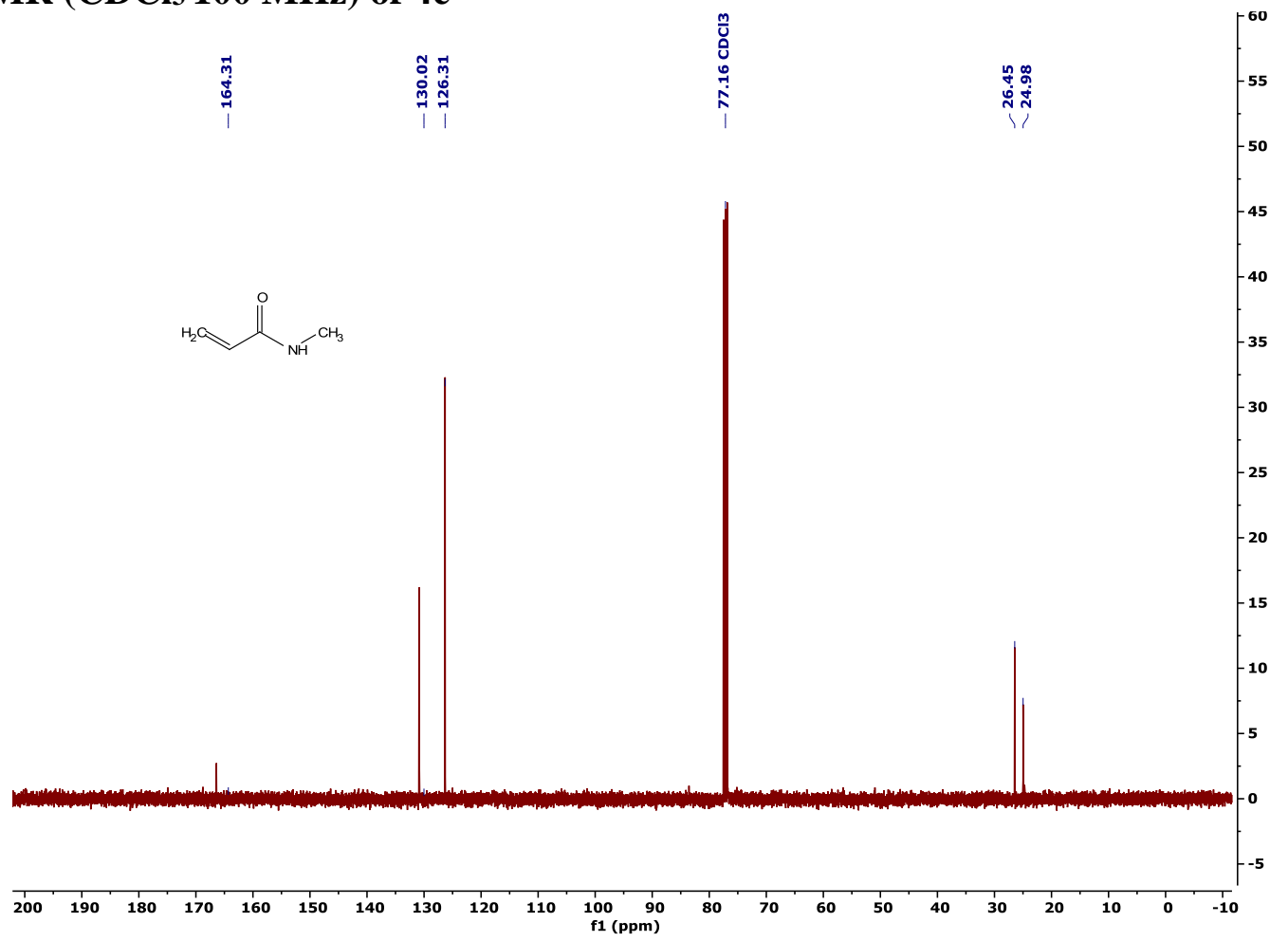


${ }^{1} \mathrm{H}$ NMR $\left(\mathrm{CDCl}_{3}, 400 \mathrm{MHz}\right)$ of $4 \mathrm{f}$

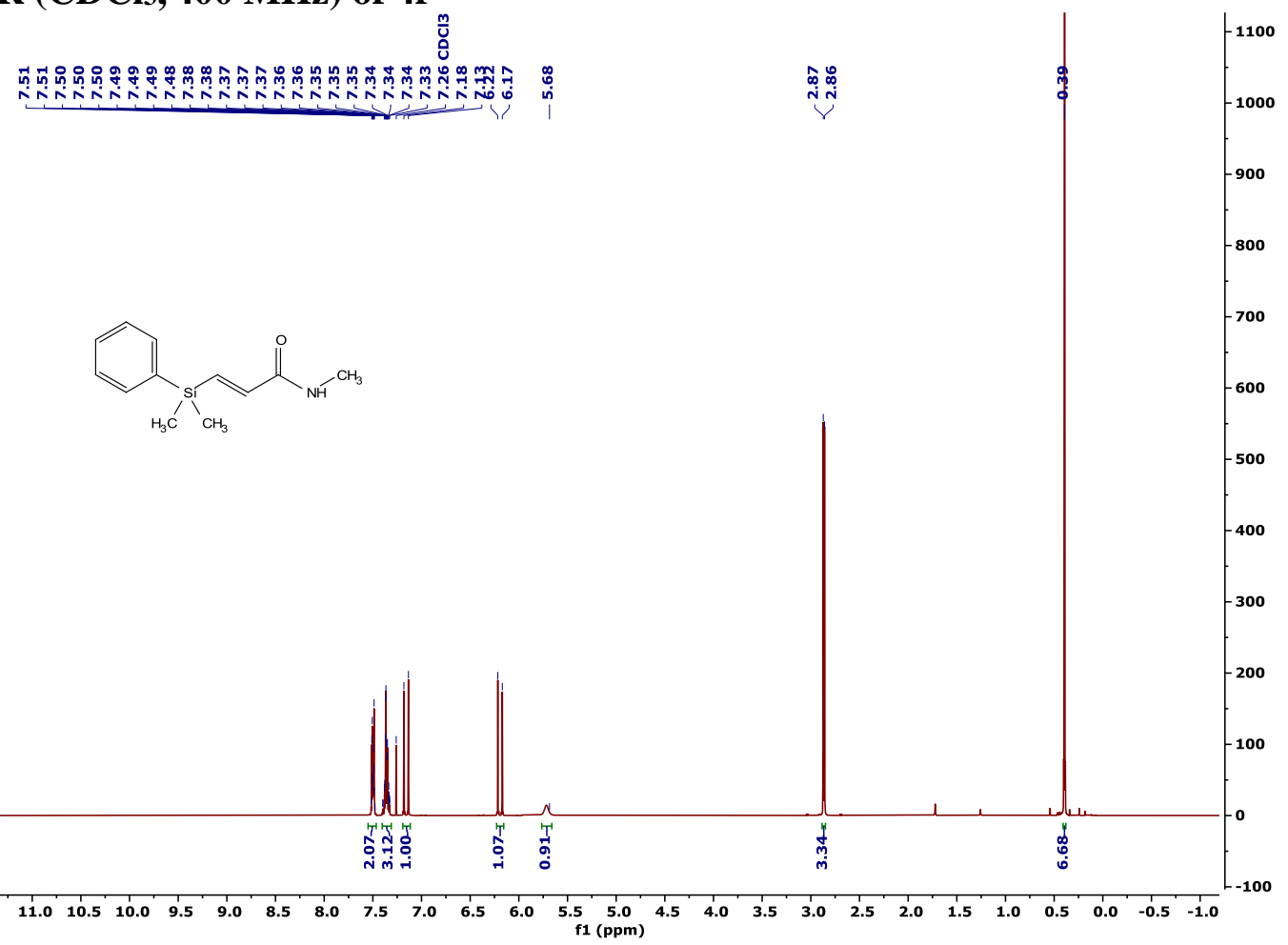

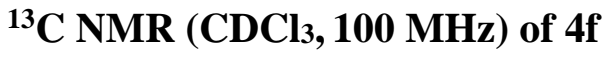

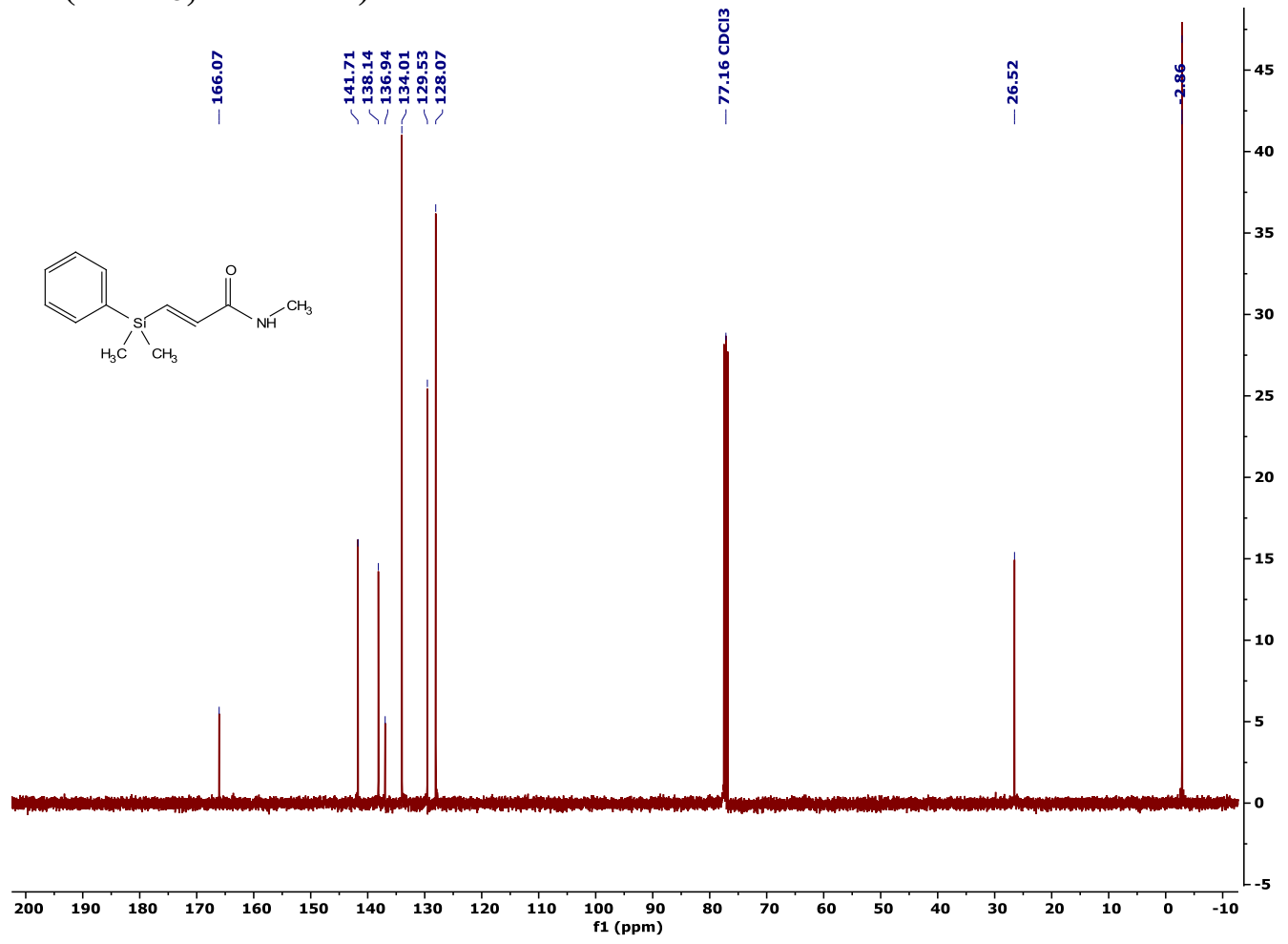


${ }^{1} \mathrm{H}$ NMR $\left(\mathrm{CDCl}_{3} 400 \mathrm{MHz}\right)$ of $6 \mathrm{a}$

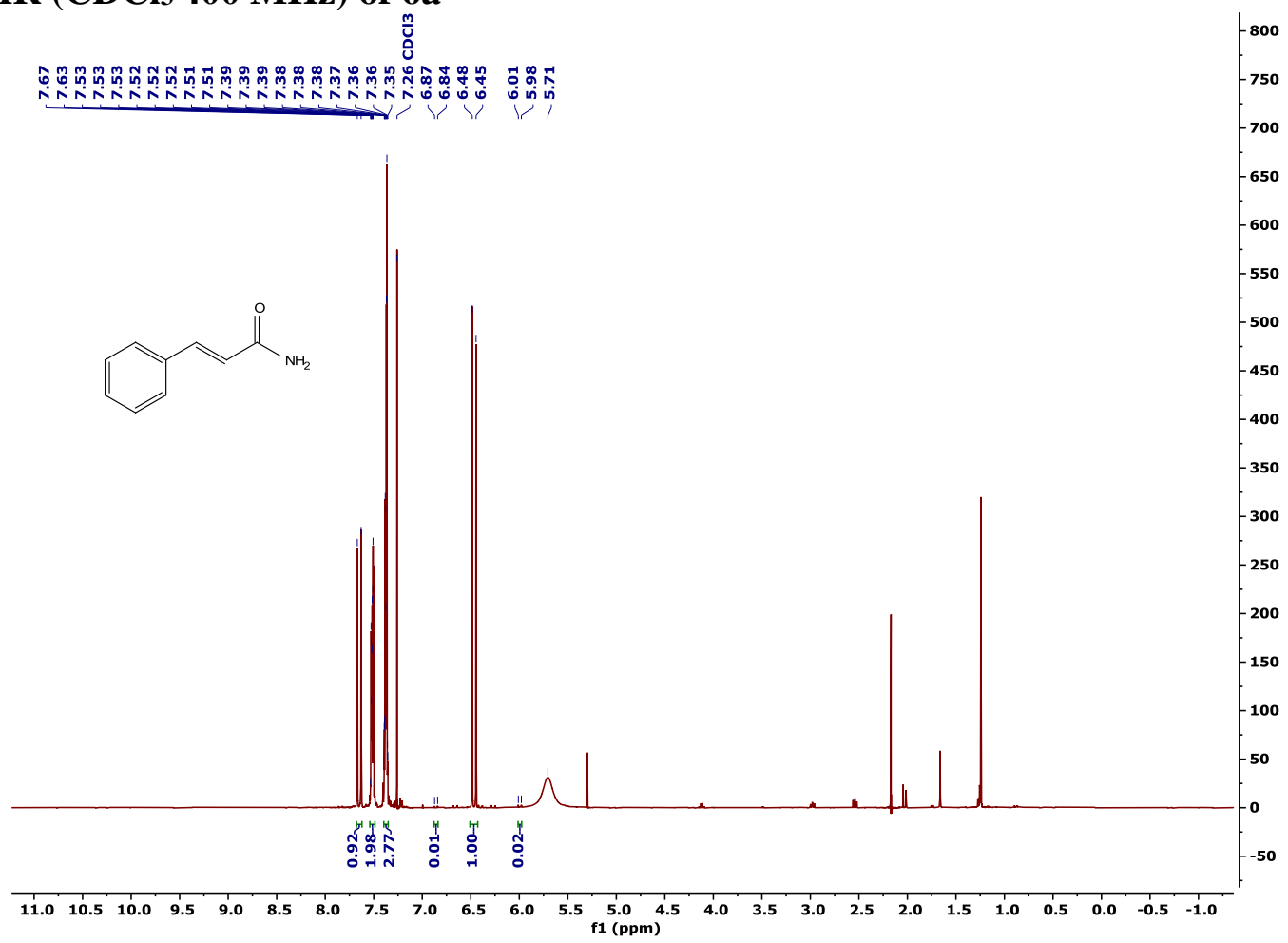

${ }^{13} \mathrm{C}$ NMR ( $\left.\mathrm{CDCl}_{3} 100 \mathrm{MHz}\right)$ of $6 \mathrm{a}$

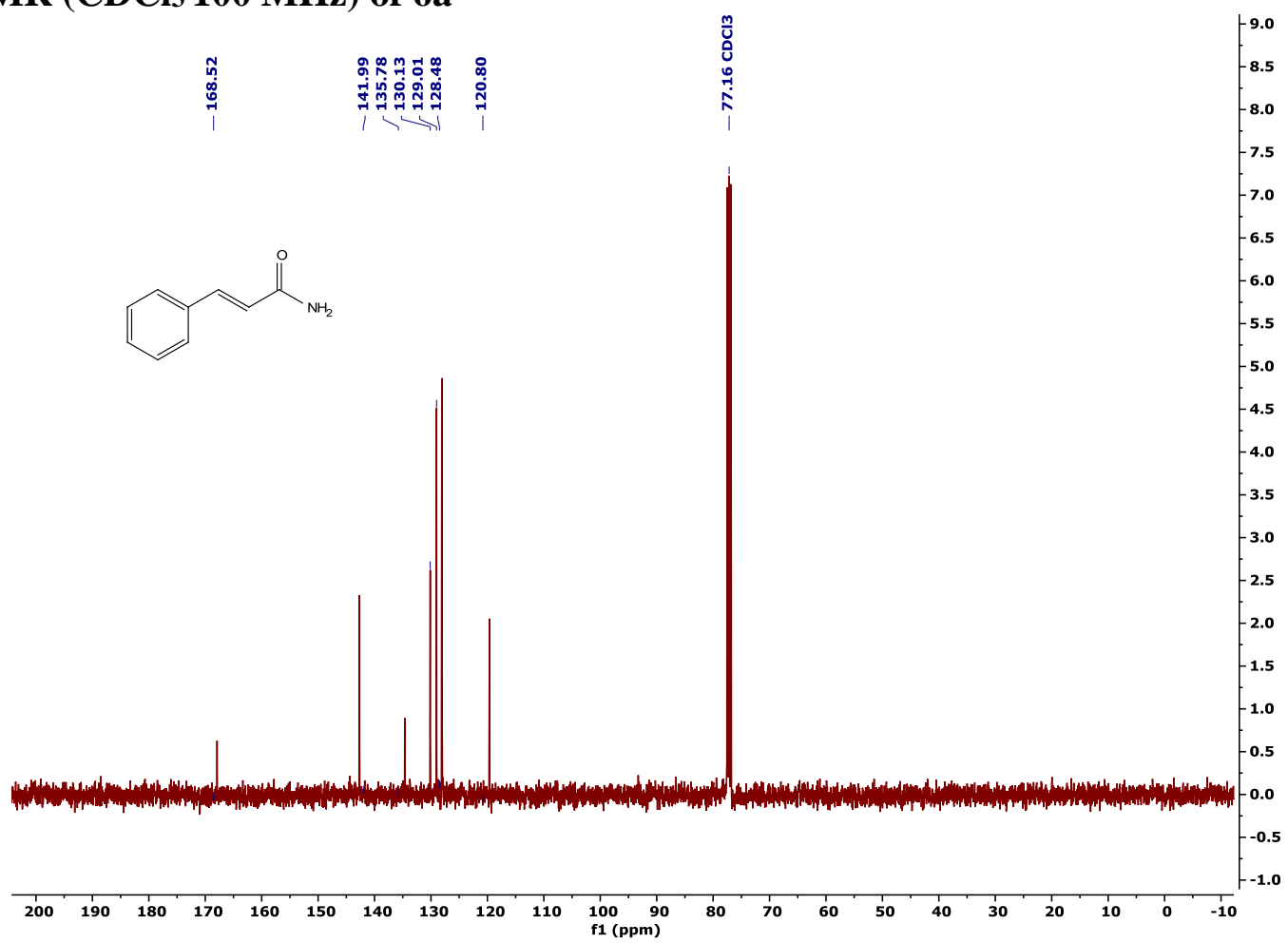


${ }^{1} \mathrm{H}$ NMR (CD $\left.30 D 400 \mathrm{MHz}\right)$ of $6 \mathrm{~b}$

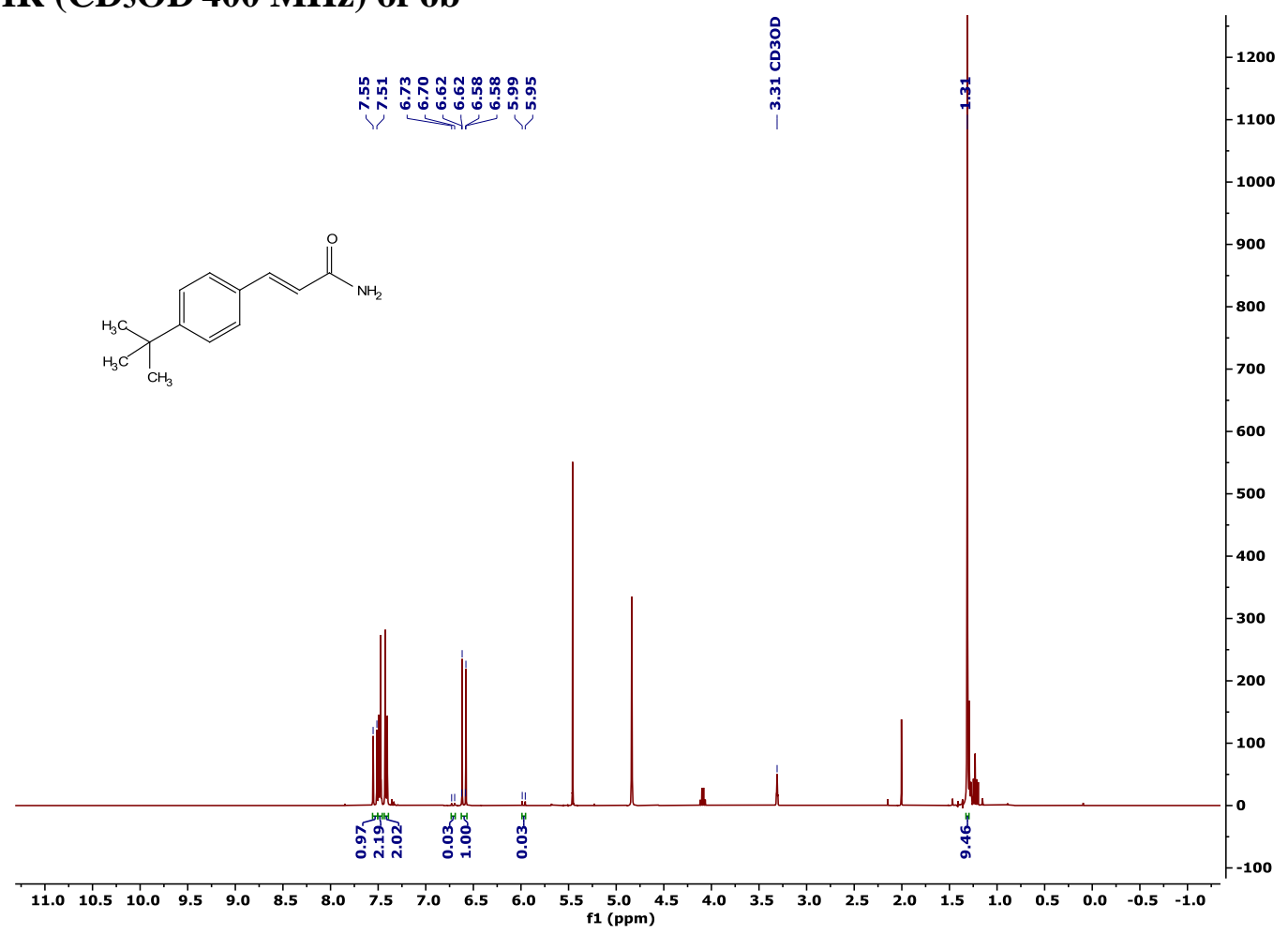

${ }^{13} \mathrm{C}$ NMR (CD $30 D 100 \mathrm{MHz}$ ) of $6 \mathrm{~b}$

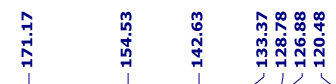
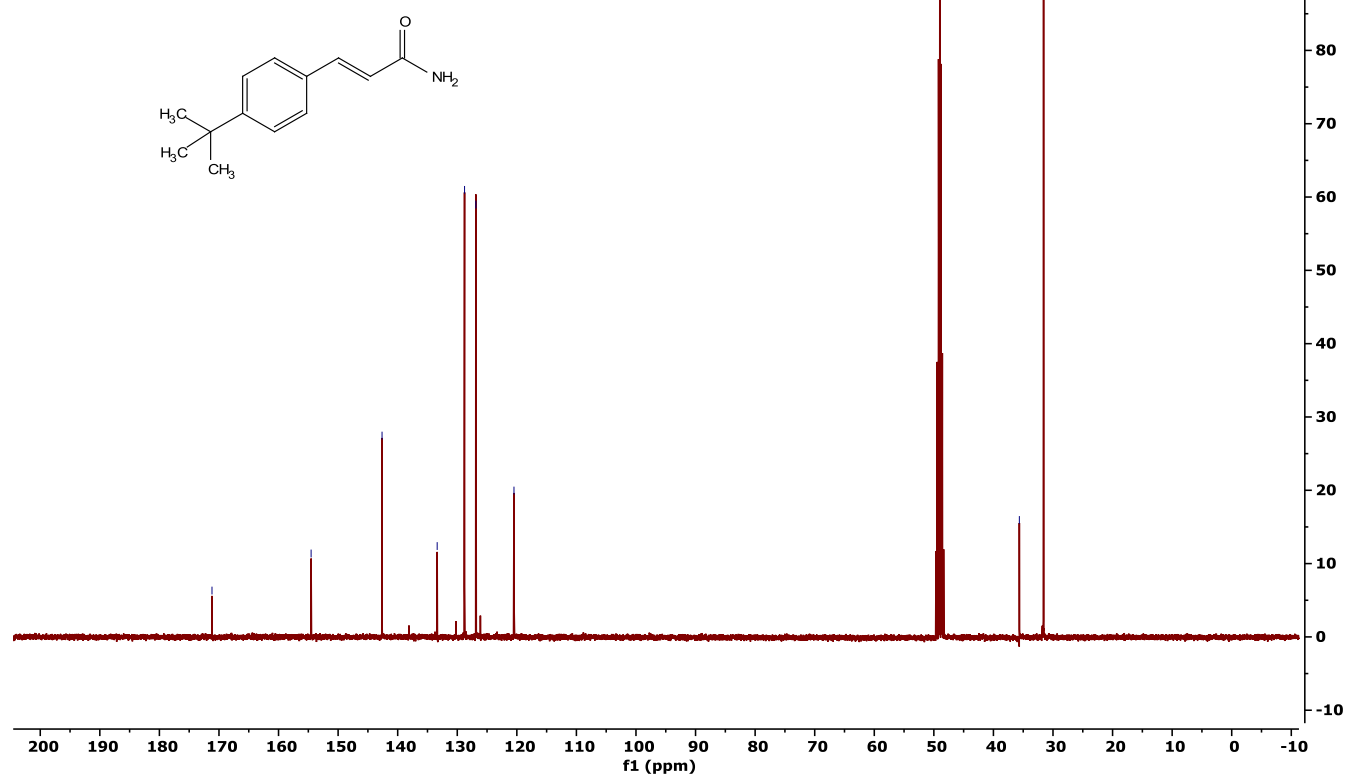
${ }^{1} \mathrm{H}$ NMR (CD3OD $\left.400 \mathrm{MHz}\right)$ of $6 \mathrm{c}$

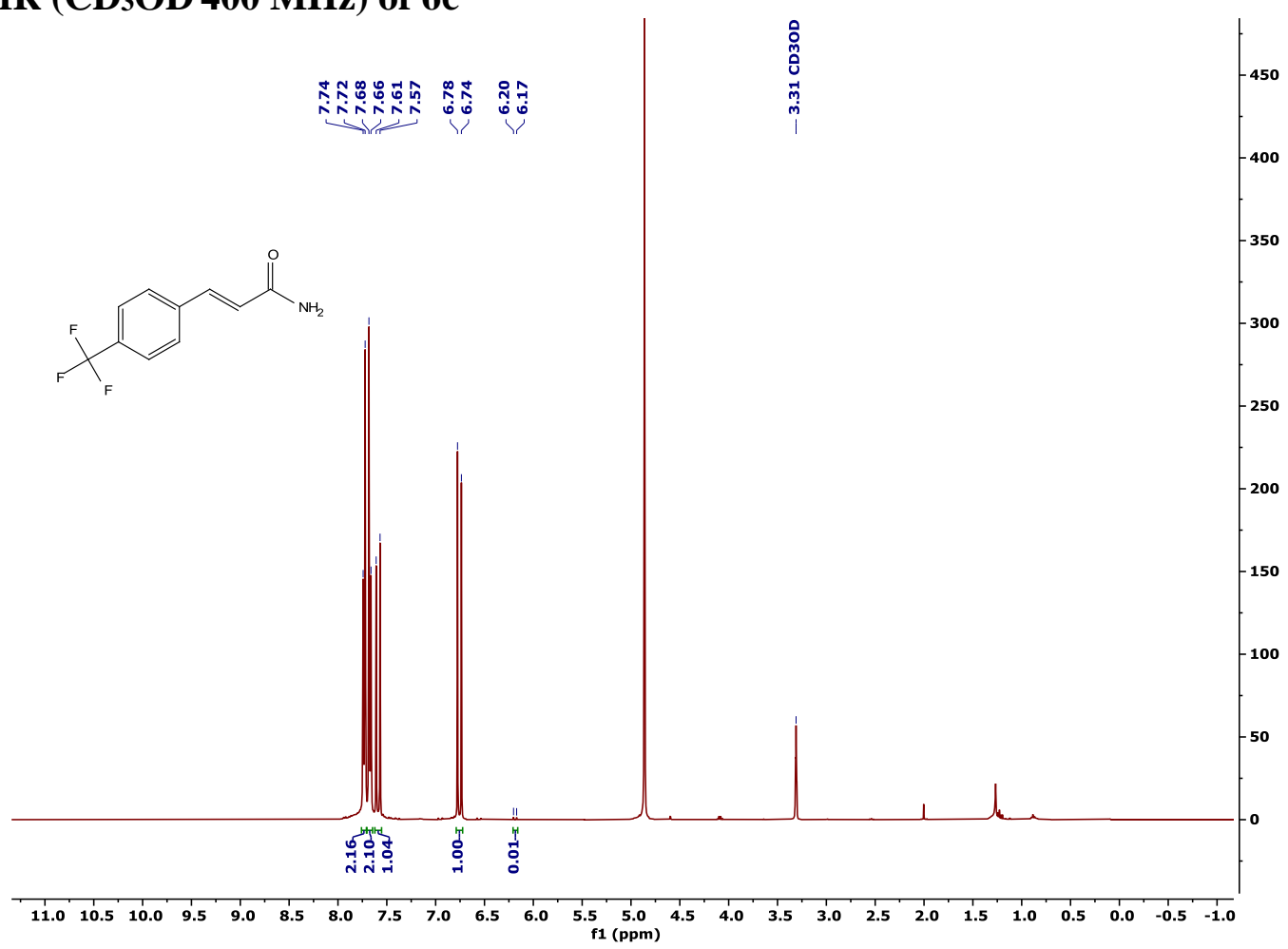

${ }^{19} \mathrm{~F}$ NMR (CD3OD $376 \mathrm{MHz}$ ) of $6 \mathrm{c}$

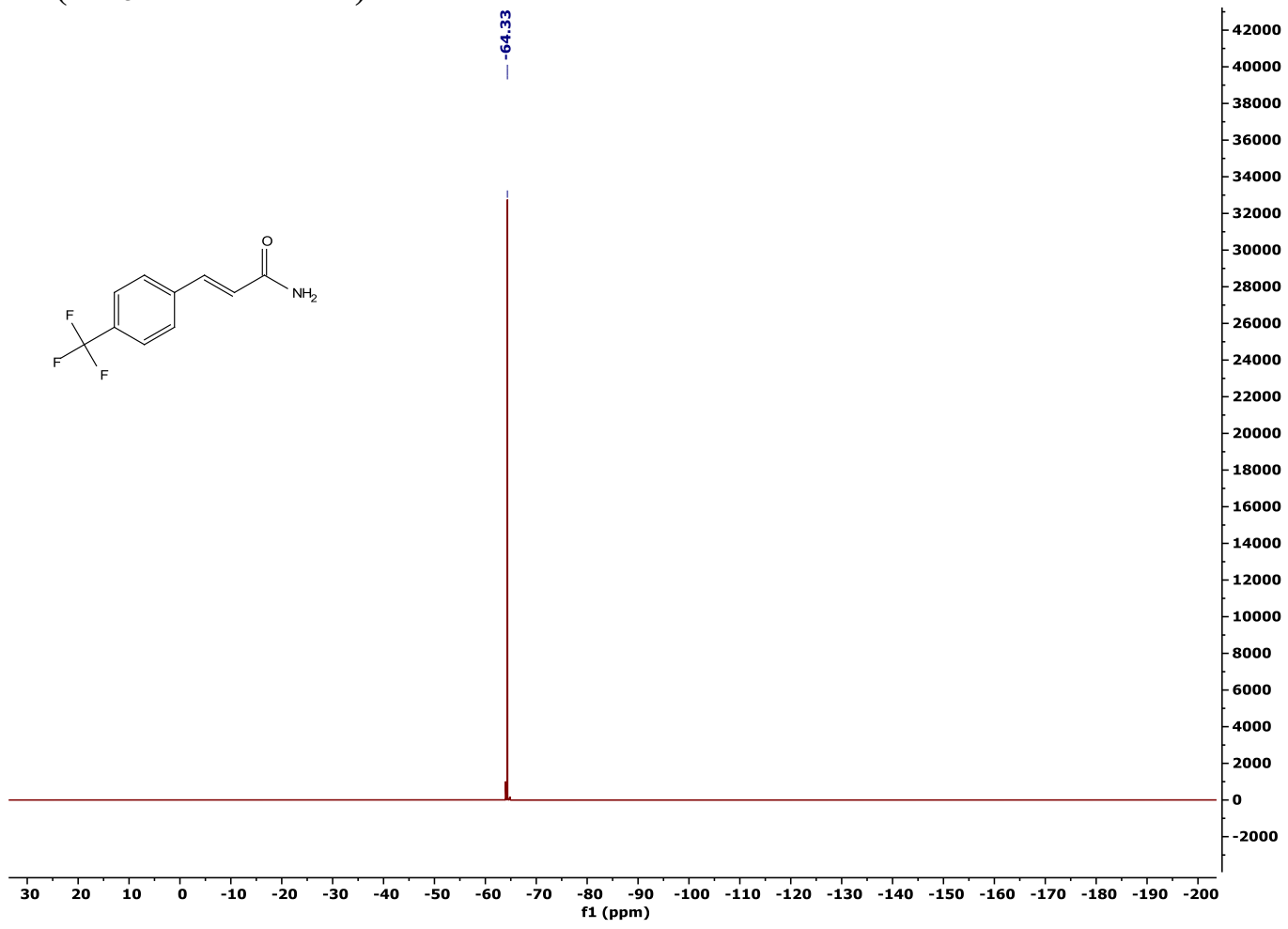


${ }^{13} \mathrm{C}$ NMR (CD3OD $100 \mathrm{MHz}$ ) of $6 \mathrm{c}$

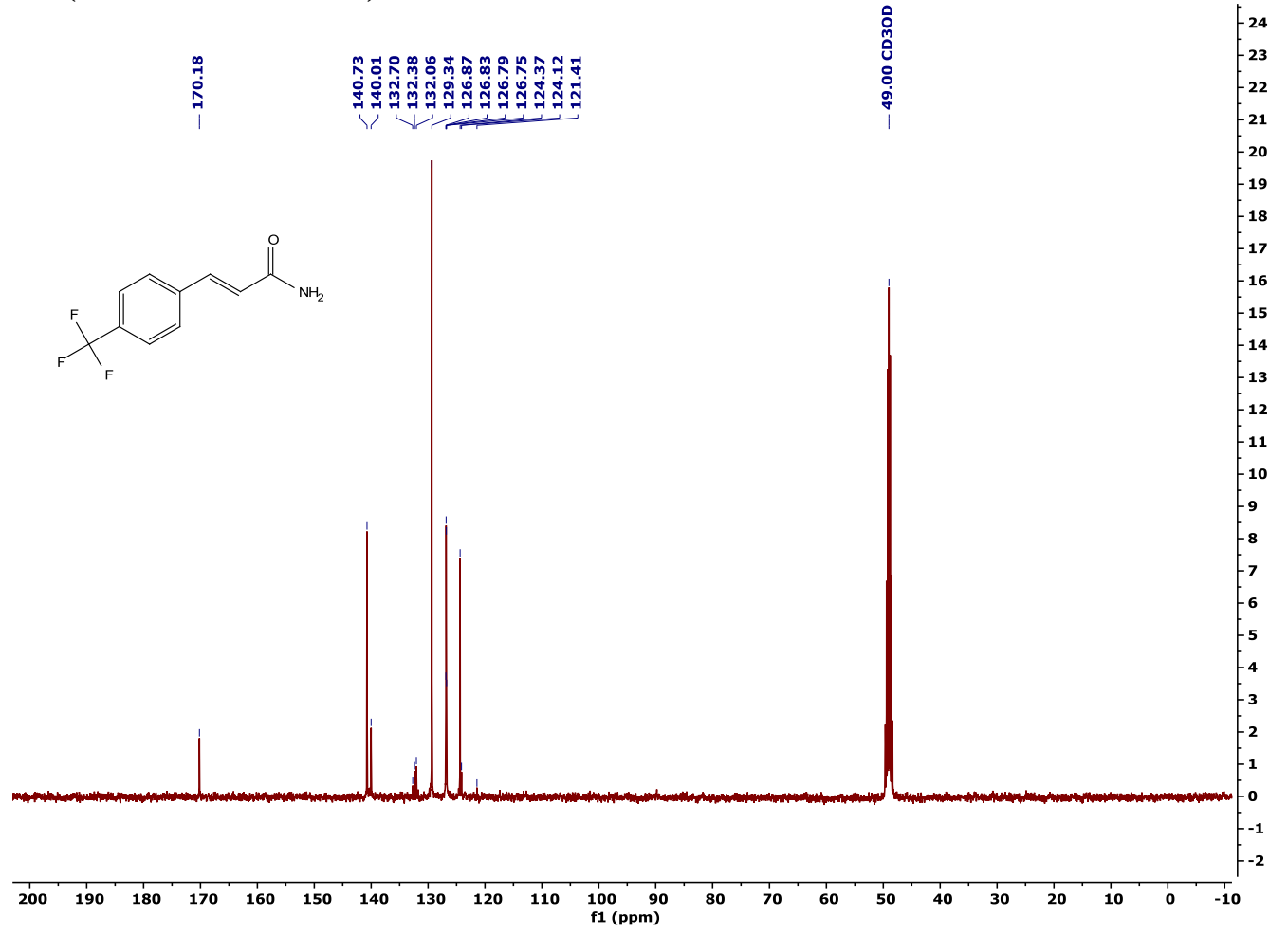

${ }^{1} \mathrm{H}$ NMR (Acetone- $d_{6} 400 \mathrm{MHz}$ ) of $6 \mathrm{~d}$

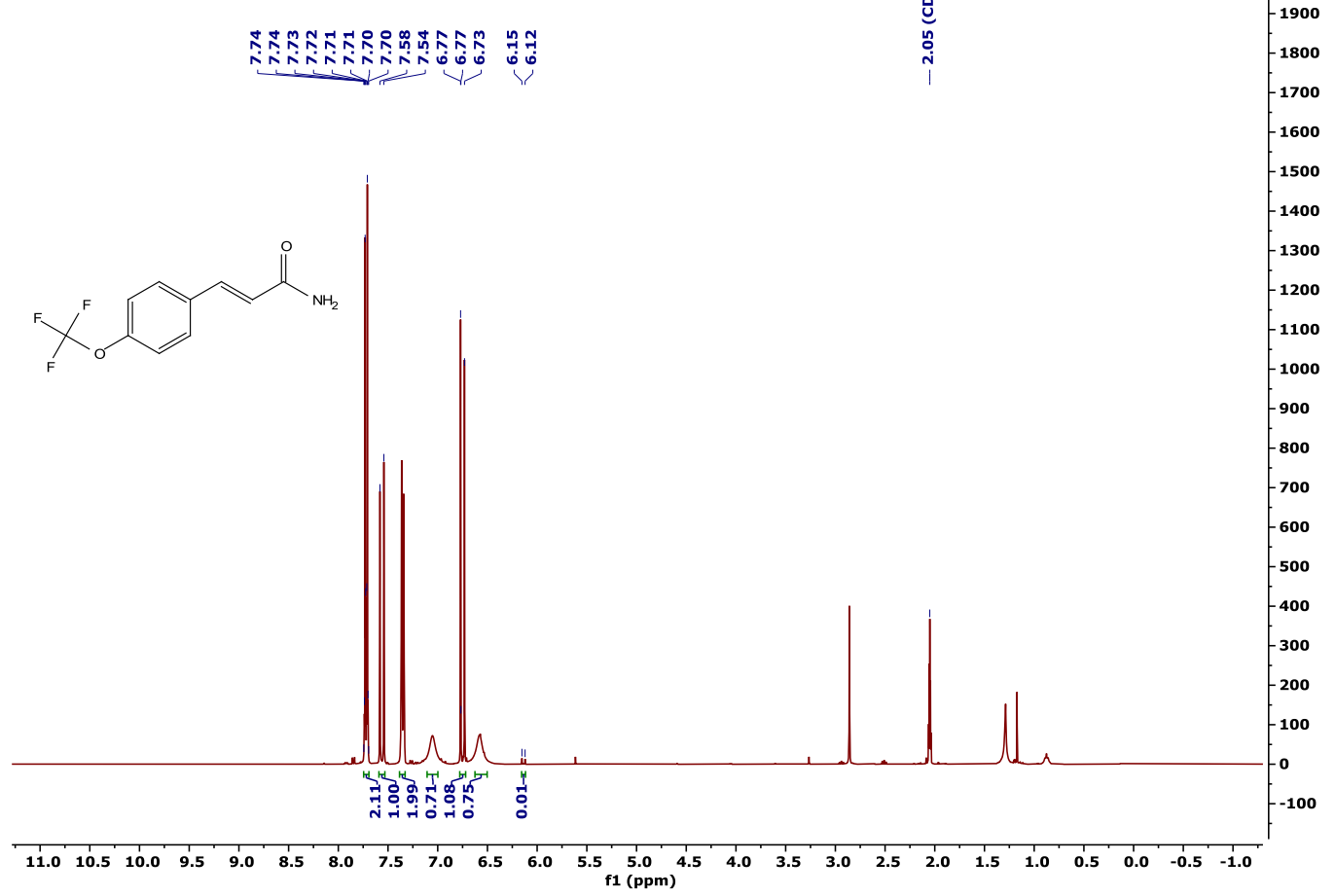


${ }^{19}$ F NMR (Acetone-d $d_{6} 376 \mathrm{MHz}$ ) of $6 \mathrm{~d}$

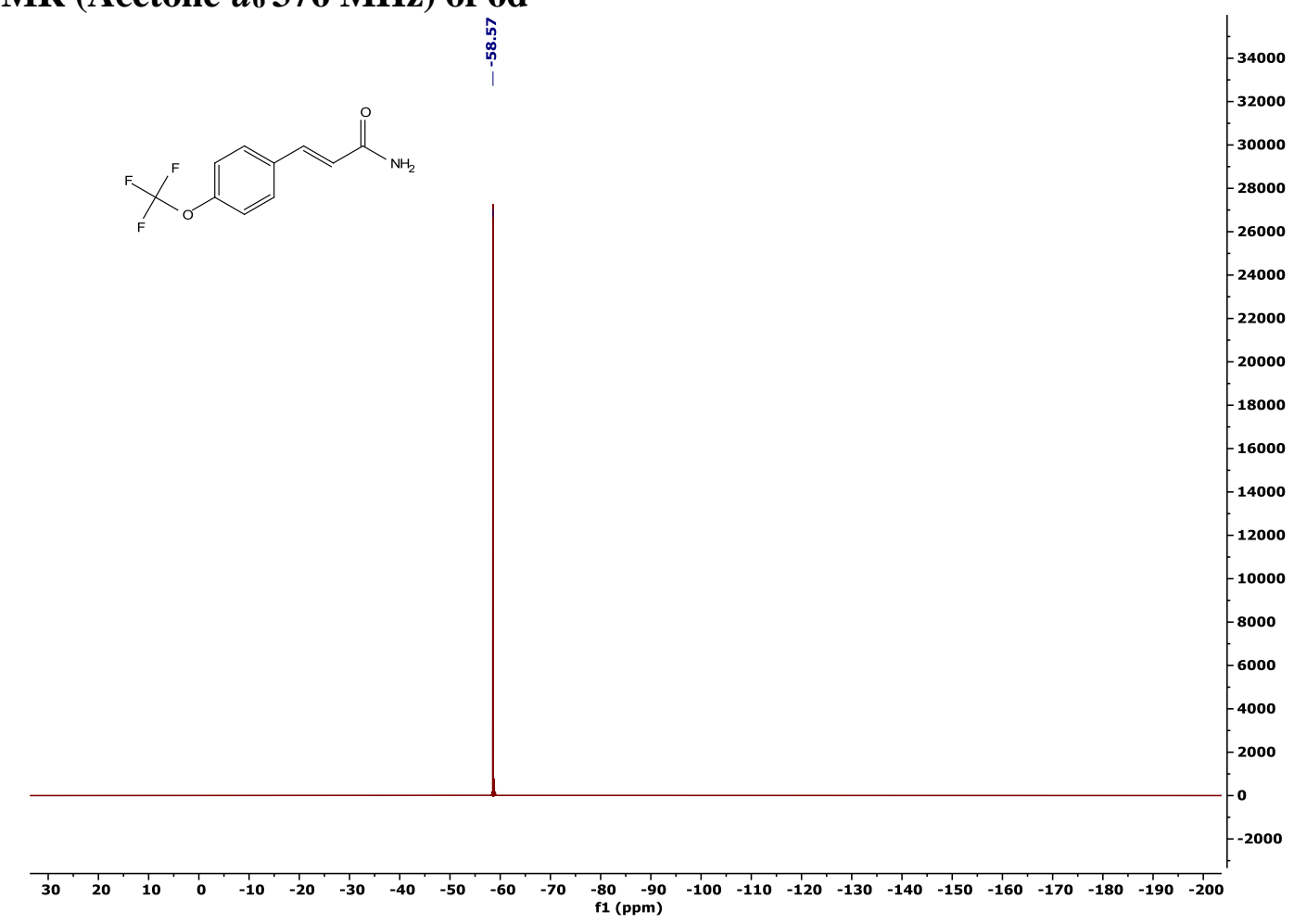

${ }^{13} \mathrm{C}$ NMR (Acetone-d $100 \mathrm{MHz}$ ) of $6 \mathrm{~d}$

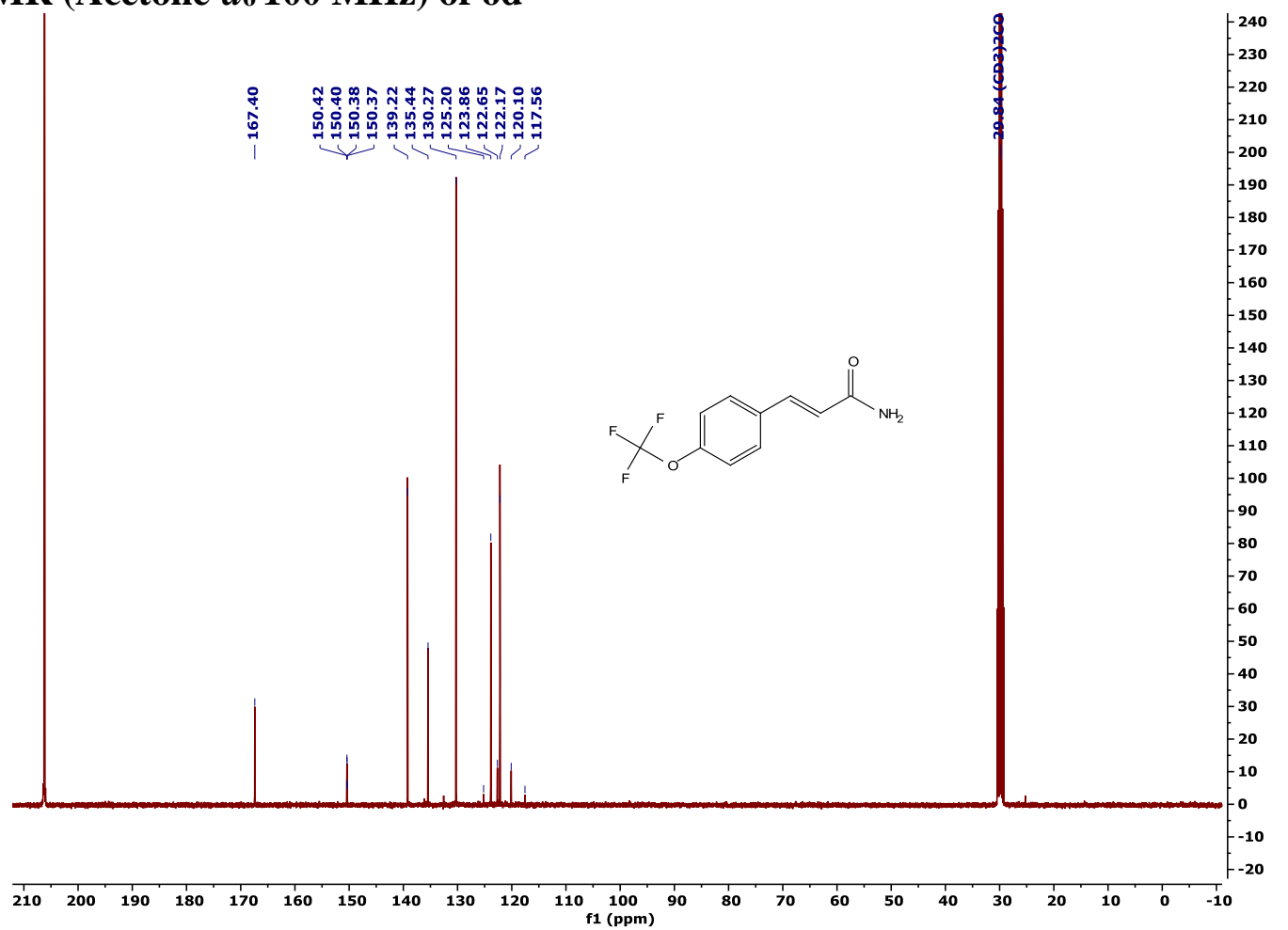


${ }^{1} \mathrm{H}$ NMR (CD3OD $\left.400 \mathrm{MHz}\right)$ of $6 \mathrm{e}$

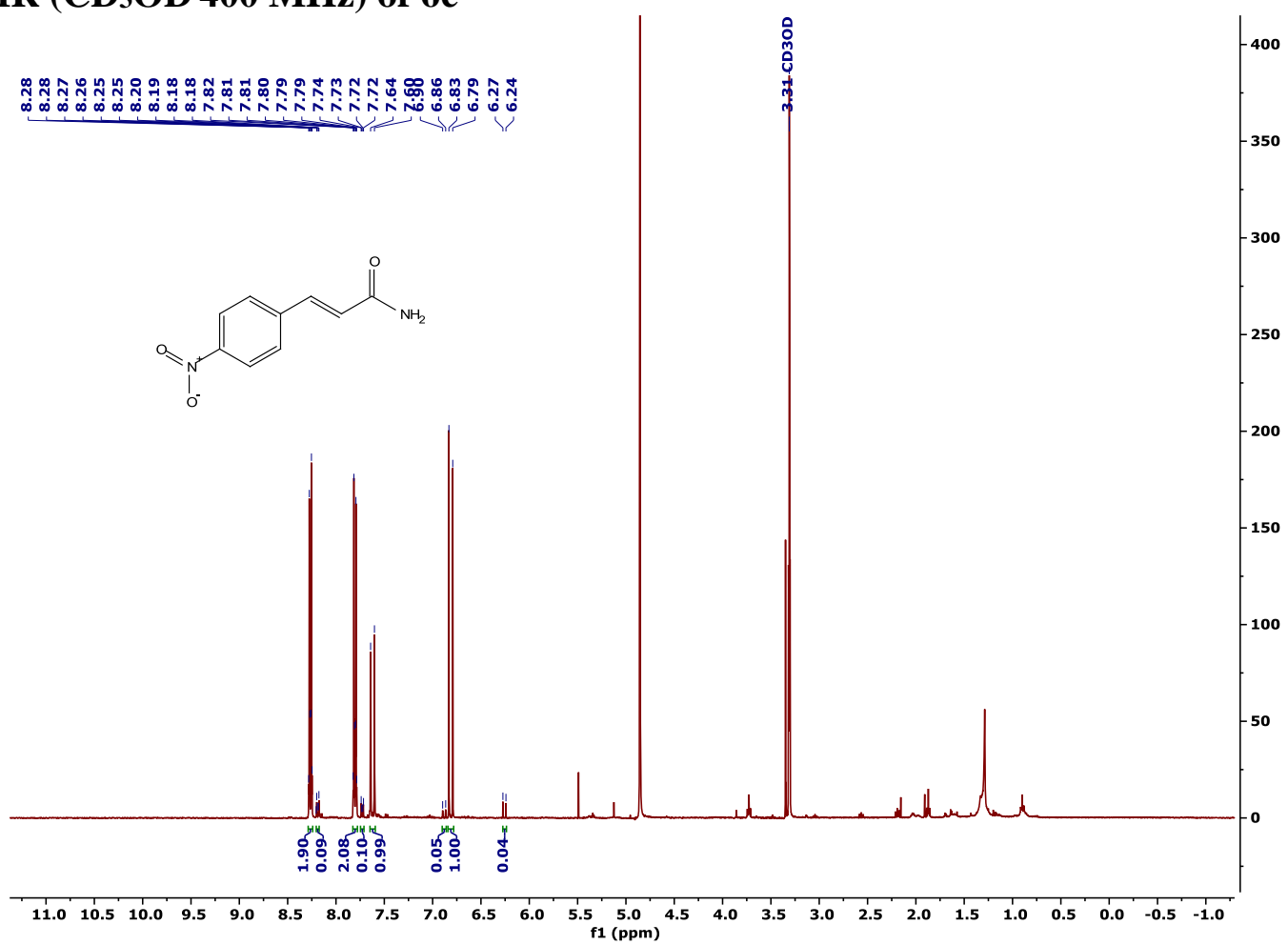

${ }^{13} \mathrm{C}$ NMR (CD3OD $126 \mathrm{MHz}$ ) of $6 \mathrm{e}$

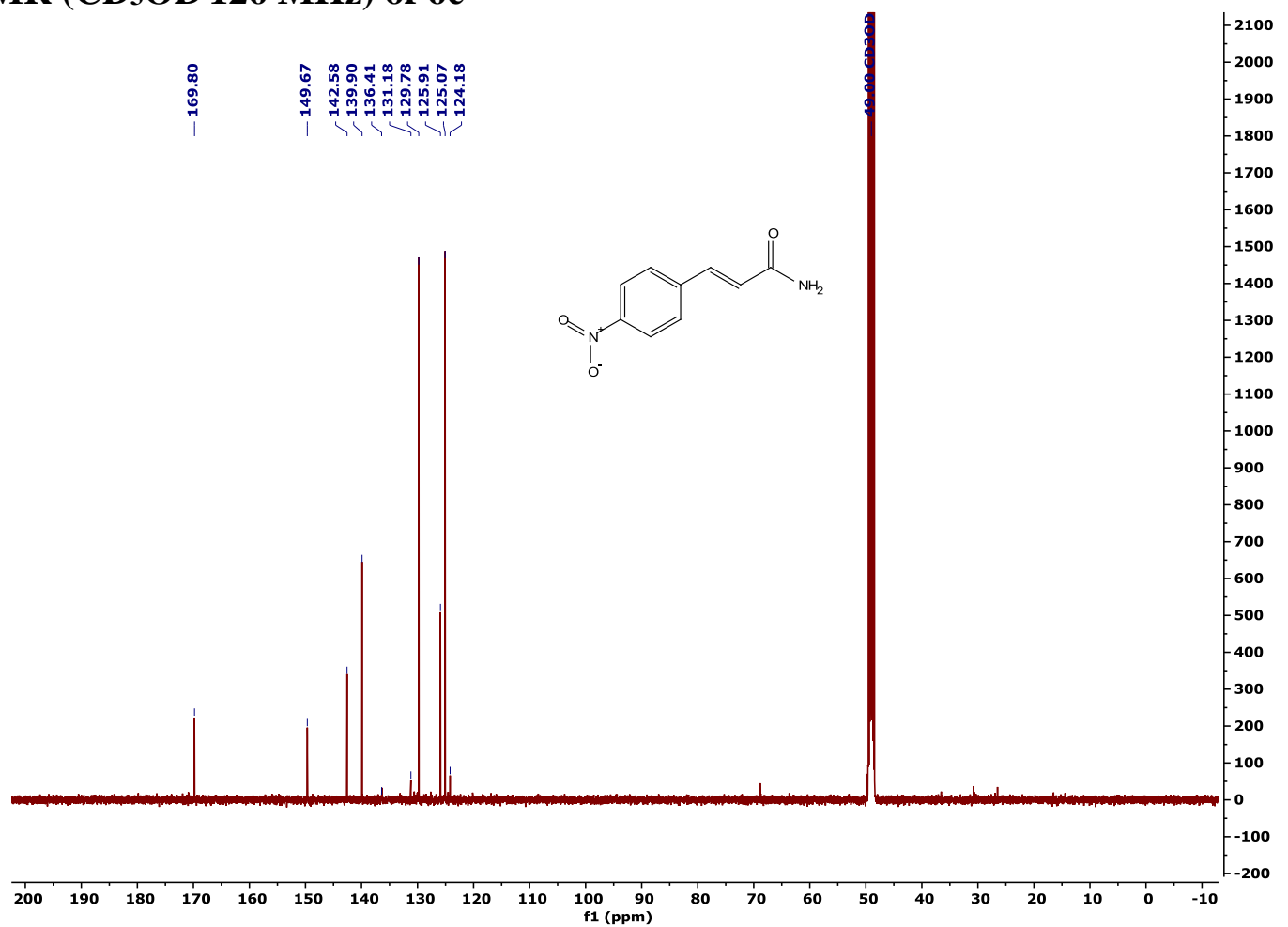




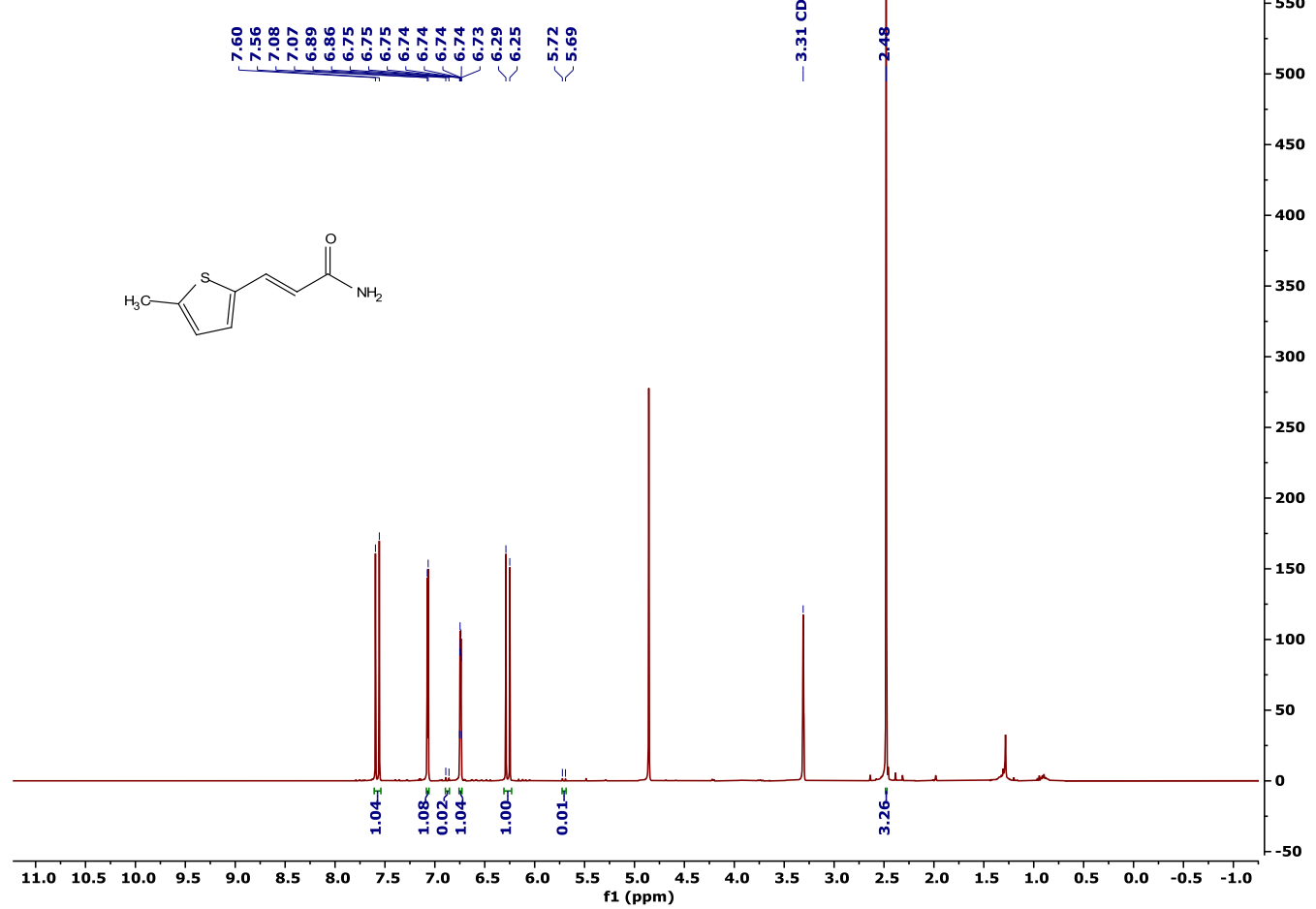

${ }^{13} \mathrm{C}$ NMR (CD3OD $100 \mathrm{MHz}$ ) of $6 f$

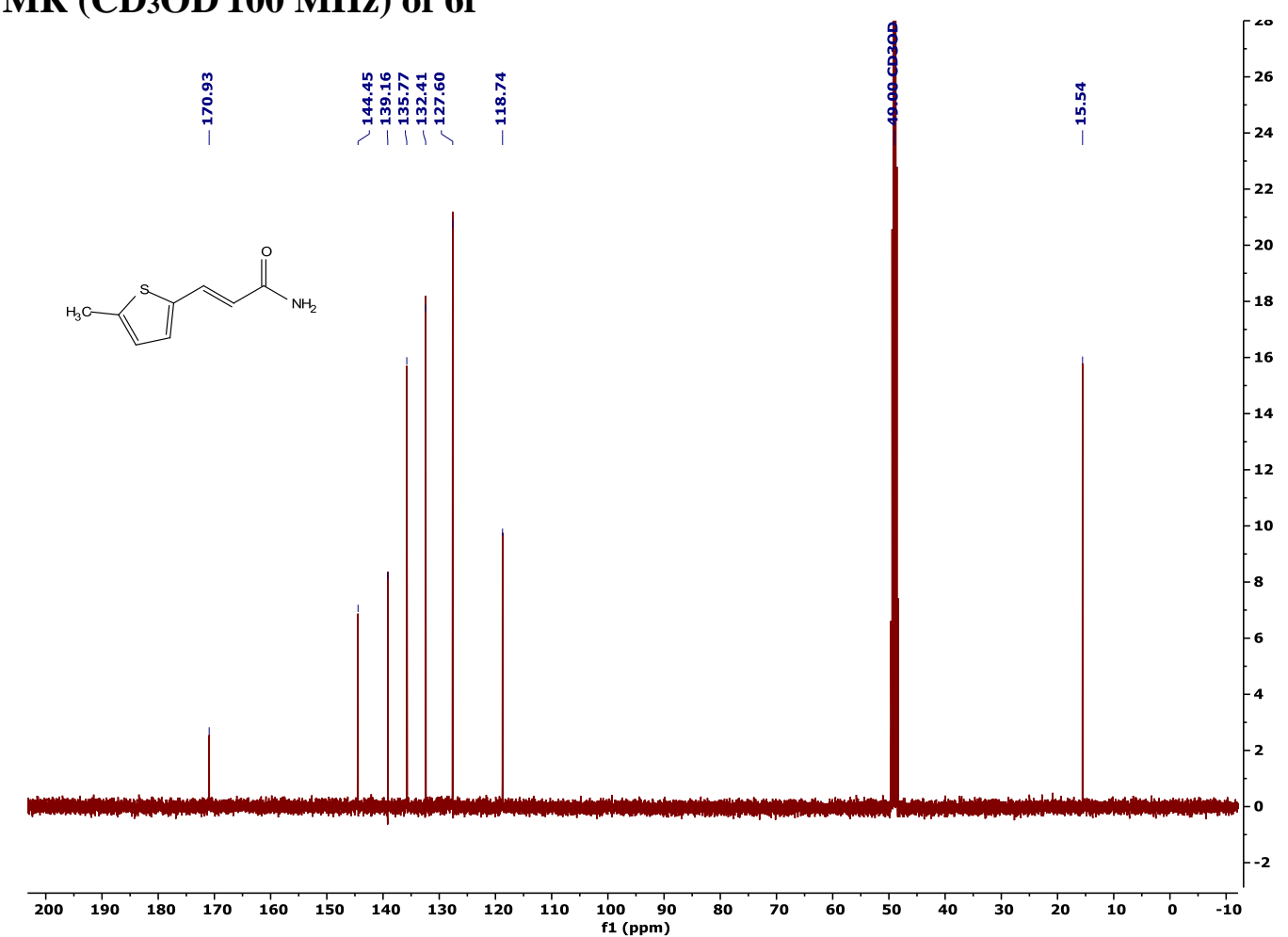


${ }^{1} \mathrm{H}$ NMR (CD3OD $\left.400 \mathrm{MHz}\right)$ of $6 \mathrm{~g}$

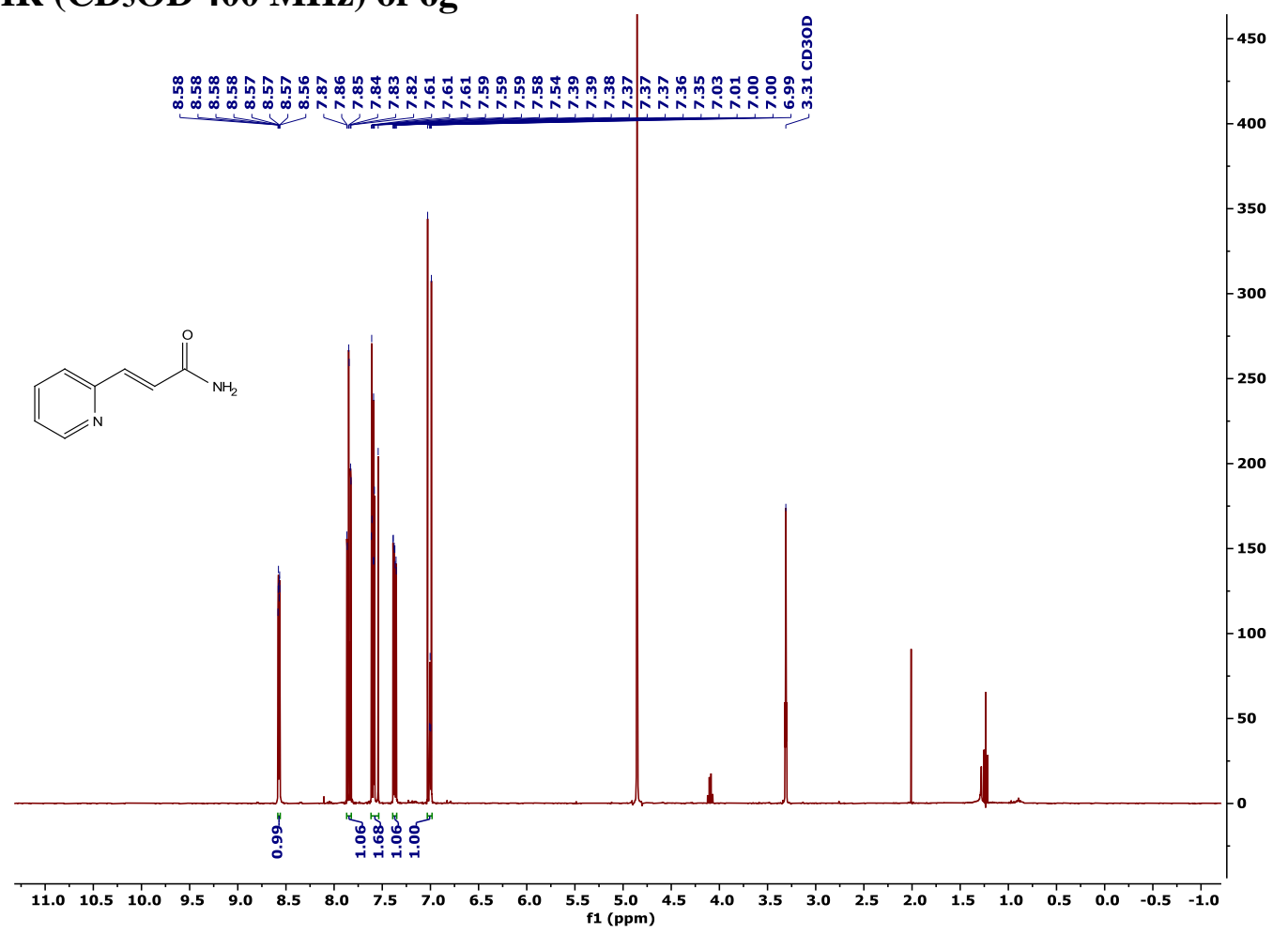

${ }^{13} \mathrm{C}$ NMR (CD3OD $100 \mathrm{MHz}$ ) of $6 \mathrm{~g}$

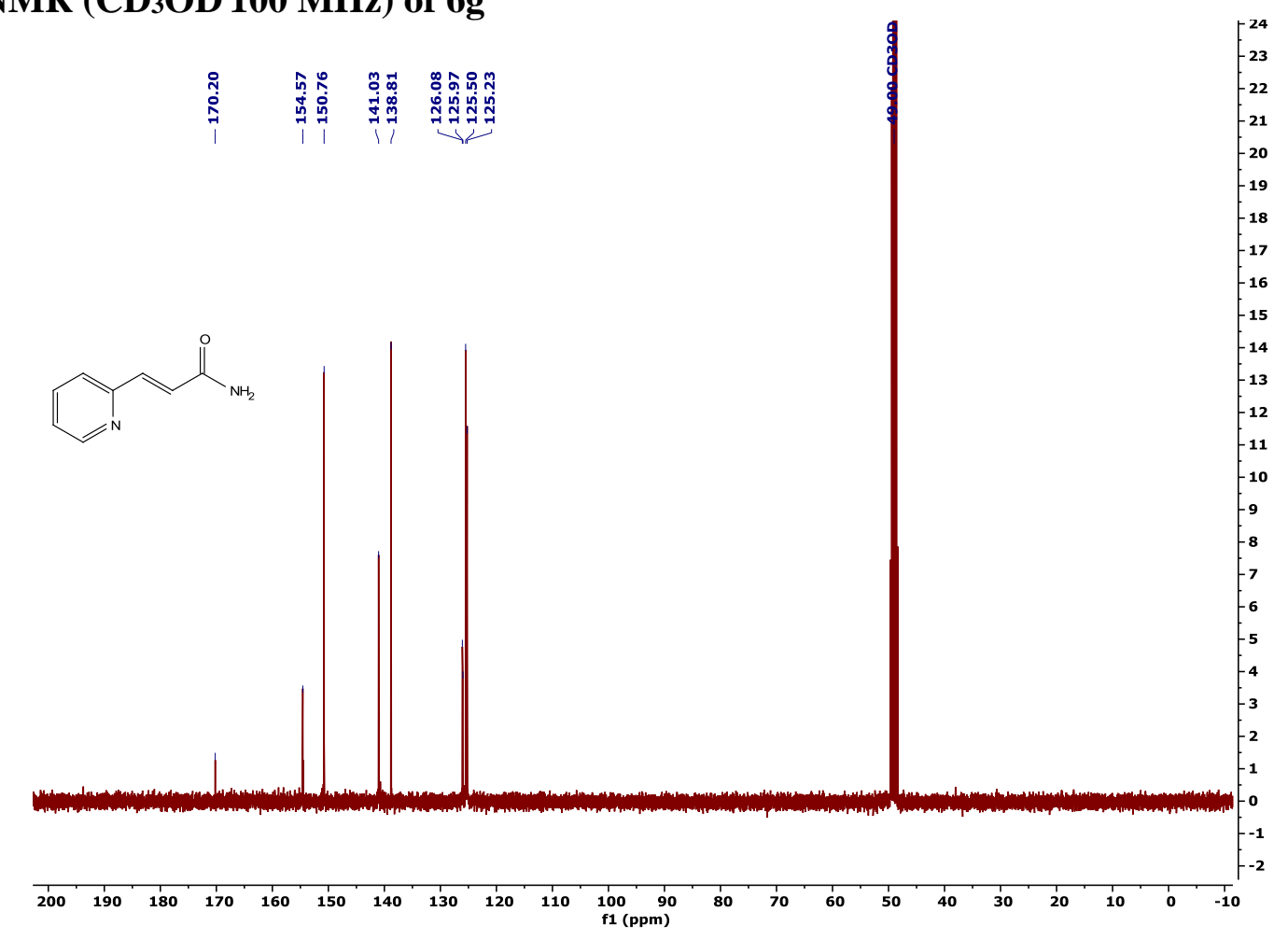




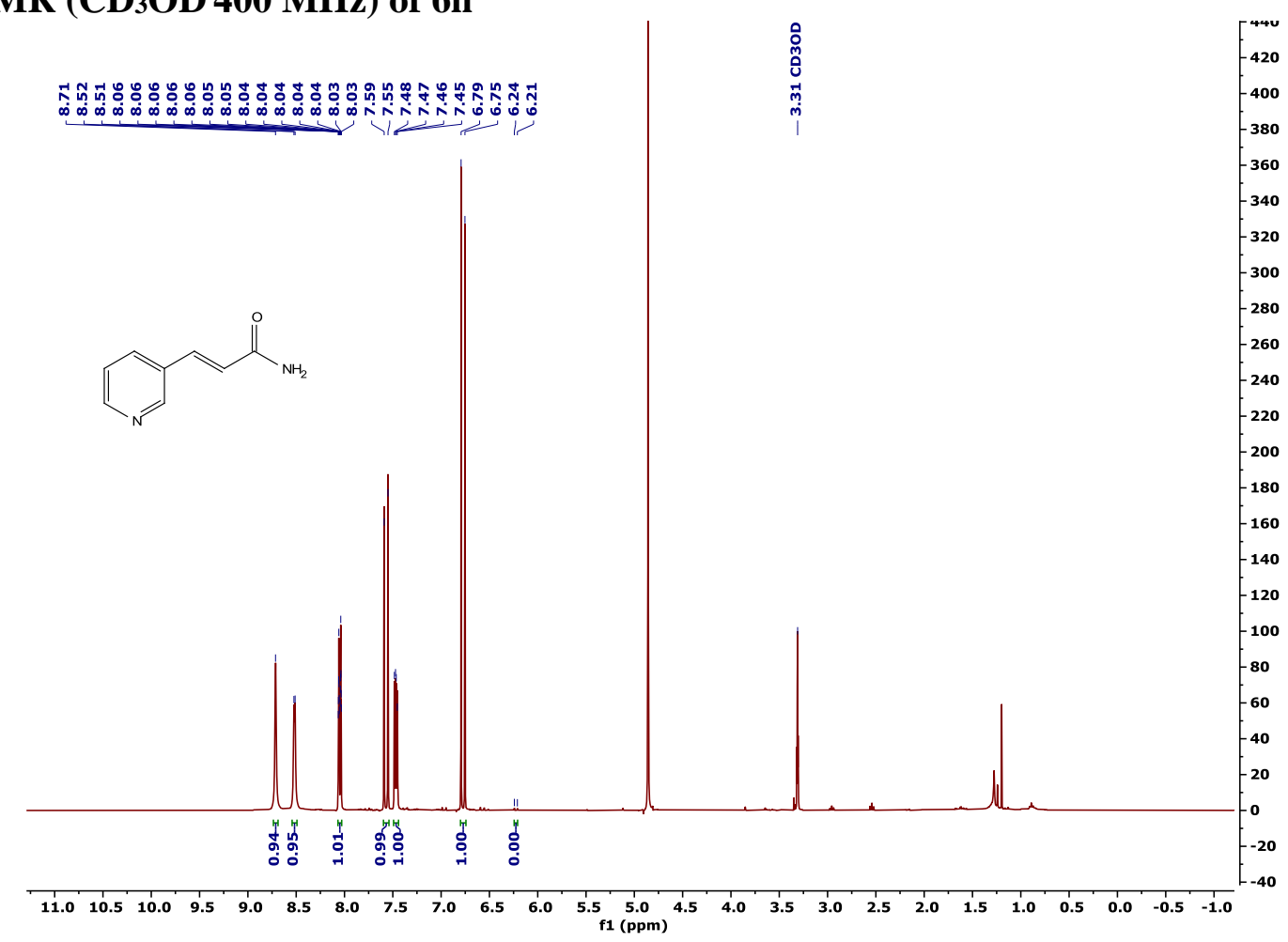

$\left.{ }^{13} \mathrm{C} \mathrm{NMR} \mathrm{(CD}{ }_{3} \mathrm{OD} 100 \mathrm{MHz}\right)$ of $6 \mathrm{~h}$

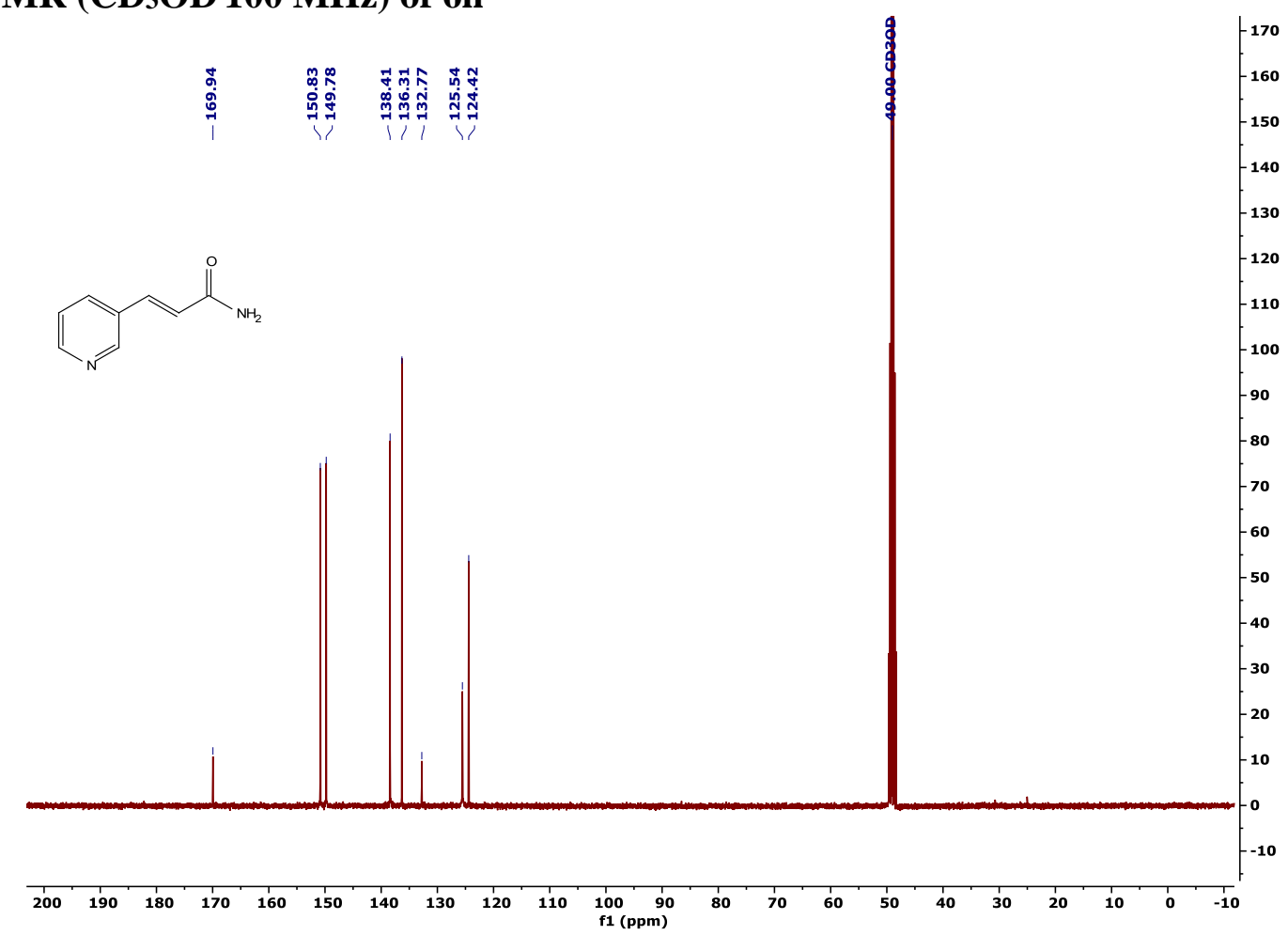


${ }^{1} \mathrm{H}$ NMR $\left(\mathrm{CD}_{3} \mathrm{OD} 400 \mathrm{MHz}\right)$ of $6 \mathrm{i}$

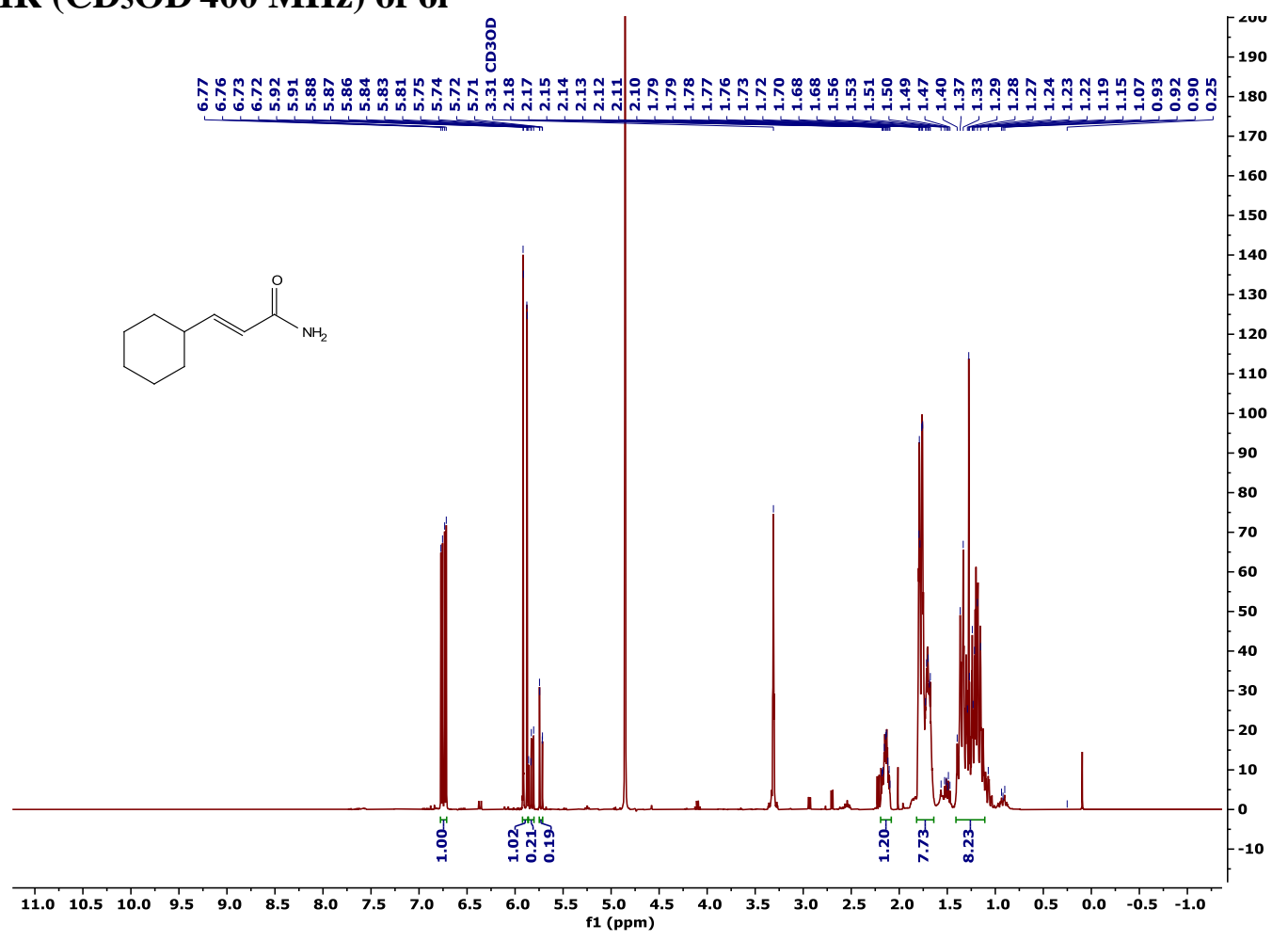

${ }^{13} \mathrm{C}$ NMR ( $\left(\mathrm{CD}_{3} \mathrm{OD} 100 \mathrm{MHz}\right)$ of $6 \mathrm{i}$

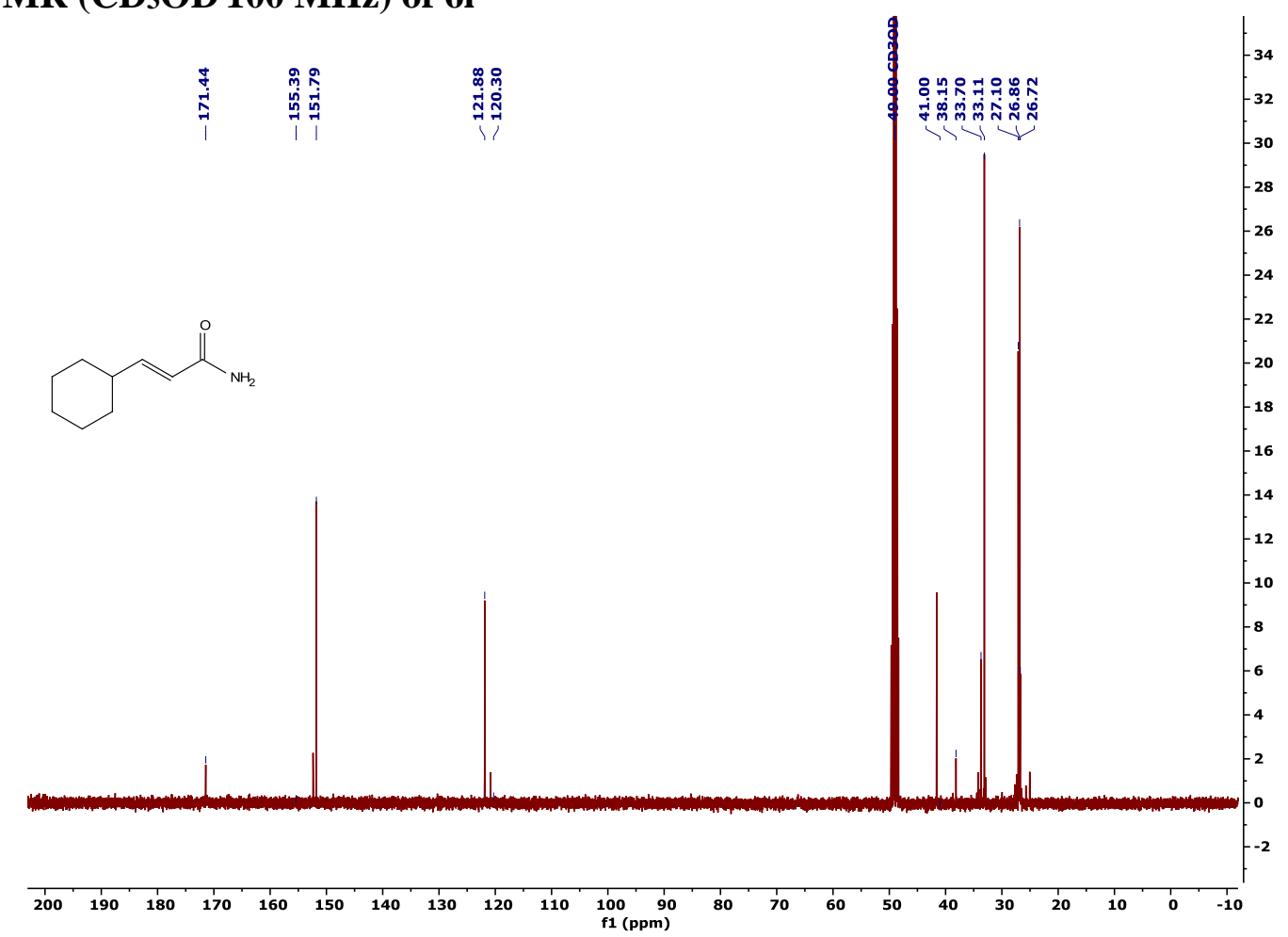


13. NMR Spectra of FK866 Analog: Intermediates and Final $E$-Cinnamamide ${ }^{1} \mathrm{H} \mathrm{NMR}\left(\mathrm{CDCl}_{3} 400 \mathrm{MHz}\right)$ of $8 \mathrm{I}$

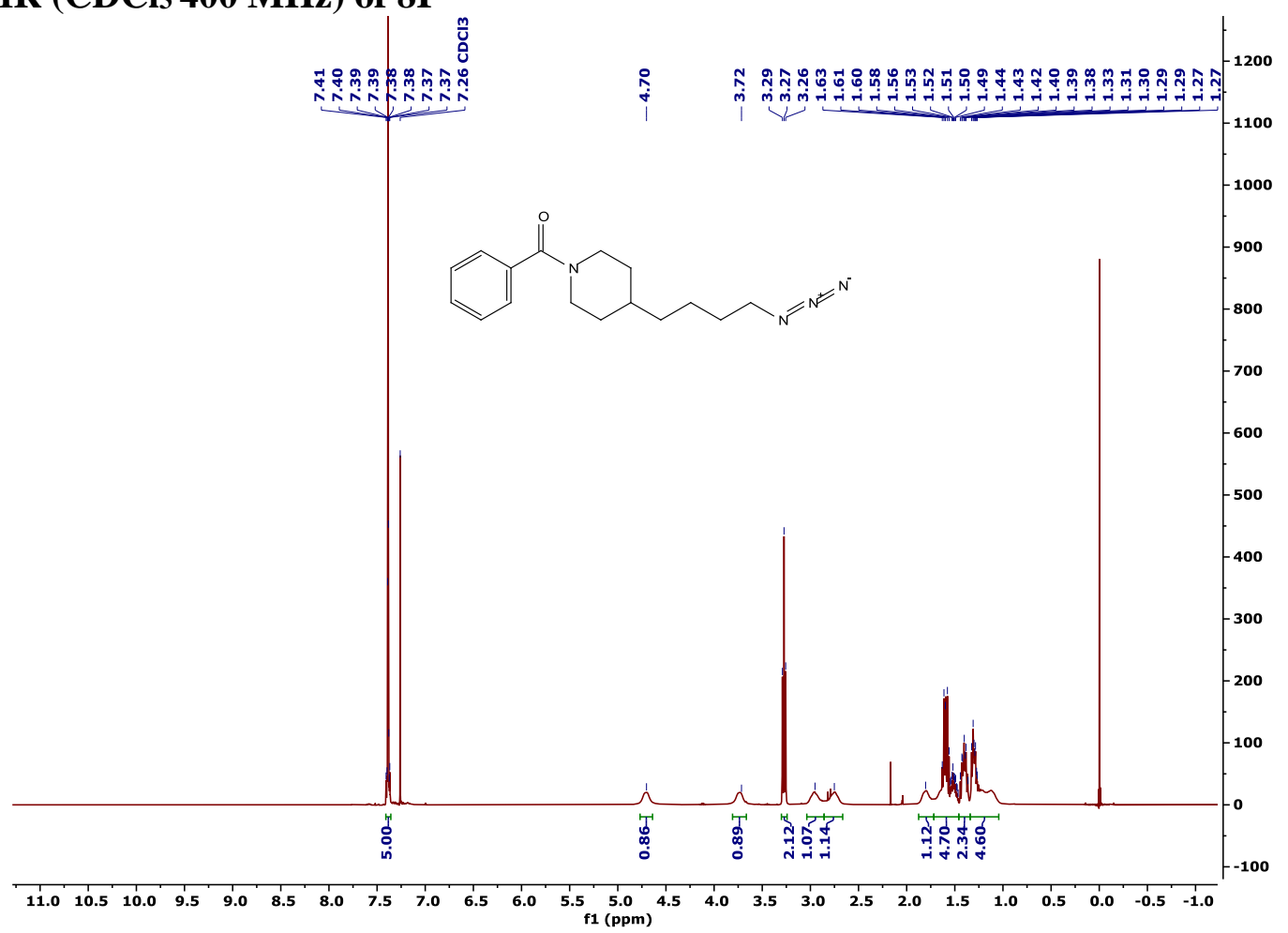

${ }^{13} \mathrm{C}$ NMR $\left(\mathrm{CDCl}_{3} 100 \mathrm{MHz}\right)$ of $8 \mathrm{I}$

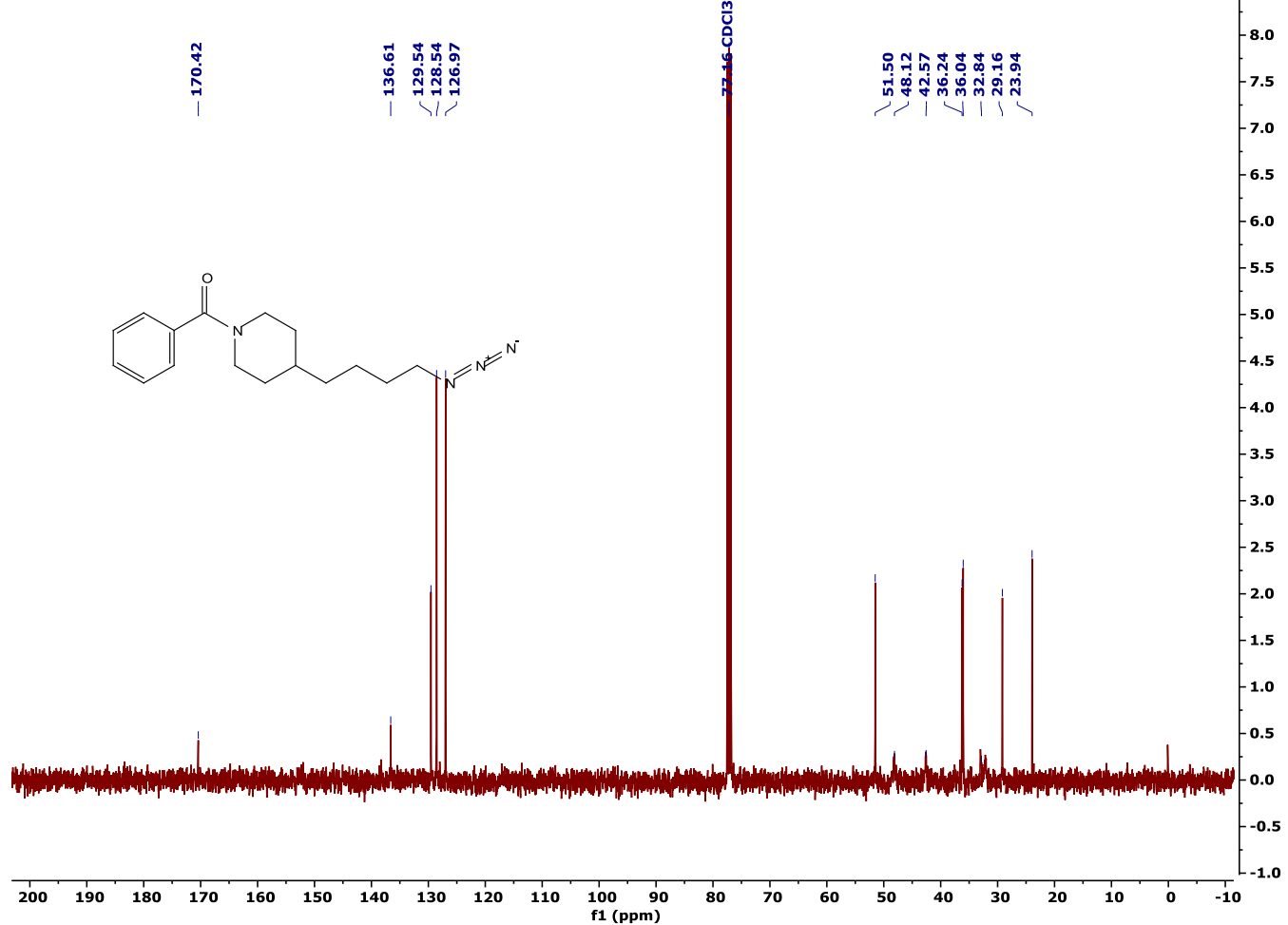


${ }^{1} \mathrm{H}$ NMR (CD $\left.{ }_{3} \mathrm{OD} 400 \mathrm{MHz}\right)$ of $8 \mathrm{II}$

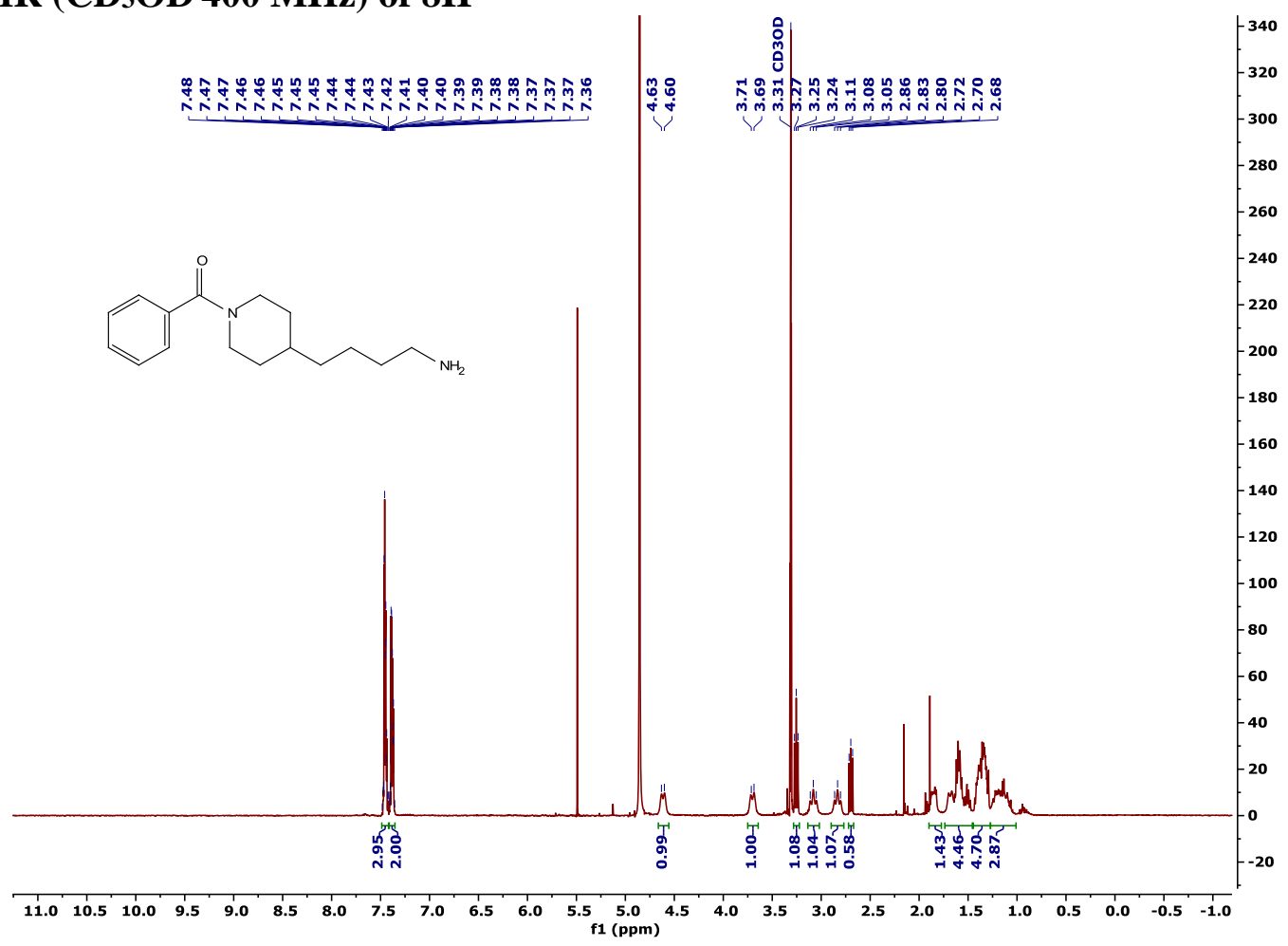

$\left.{ }^{13} \mathrm{C} \mathrm{NMR} \mathrm{(CD} \mathrm{OD} 100 \mathrm{MHz}\right)$ of $8 \mathrm{II}$
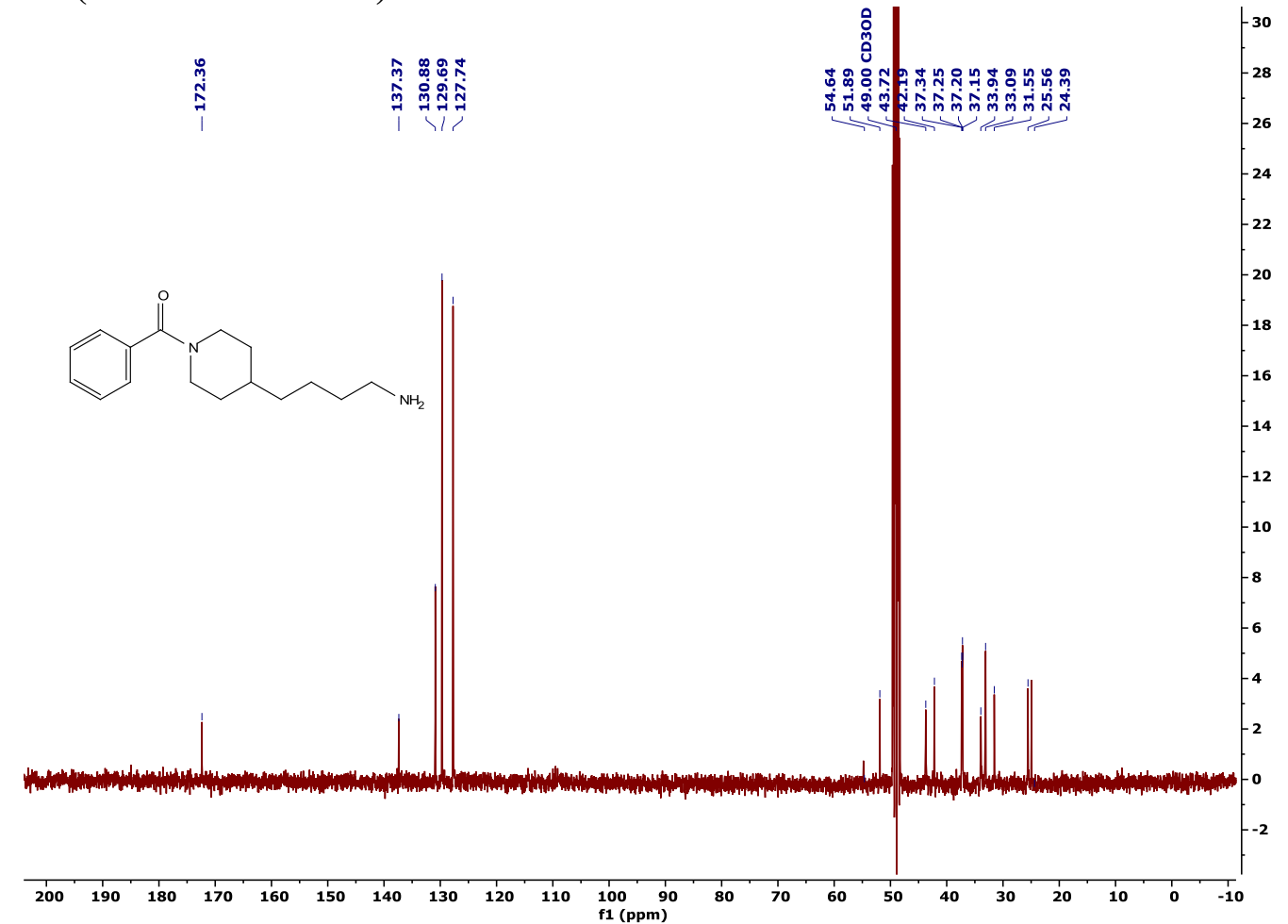

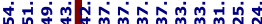
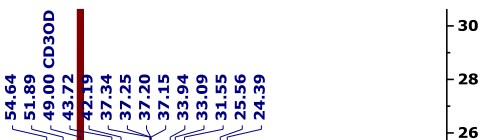

-30
-28

$-26$

$-24$

22 
${ }^{1} \mathrm{H}$ NMR (CD3 $\left.\mathrm{OD} 400 \mathrm{MHz}\right)$ of 8

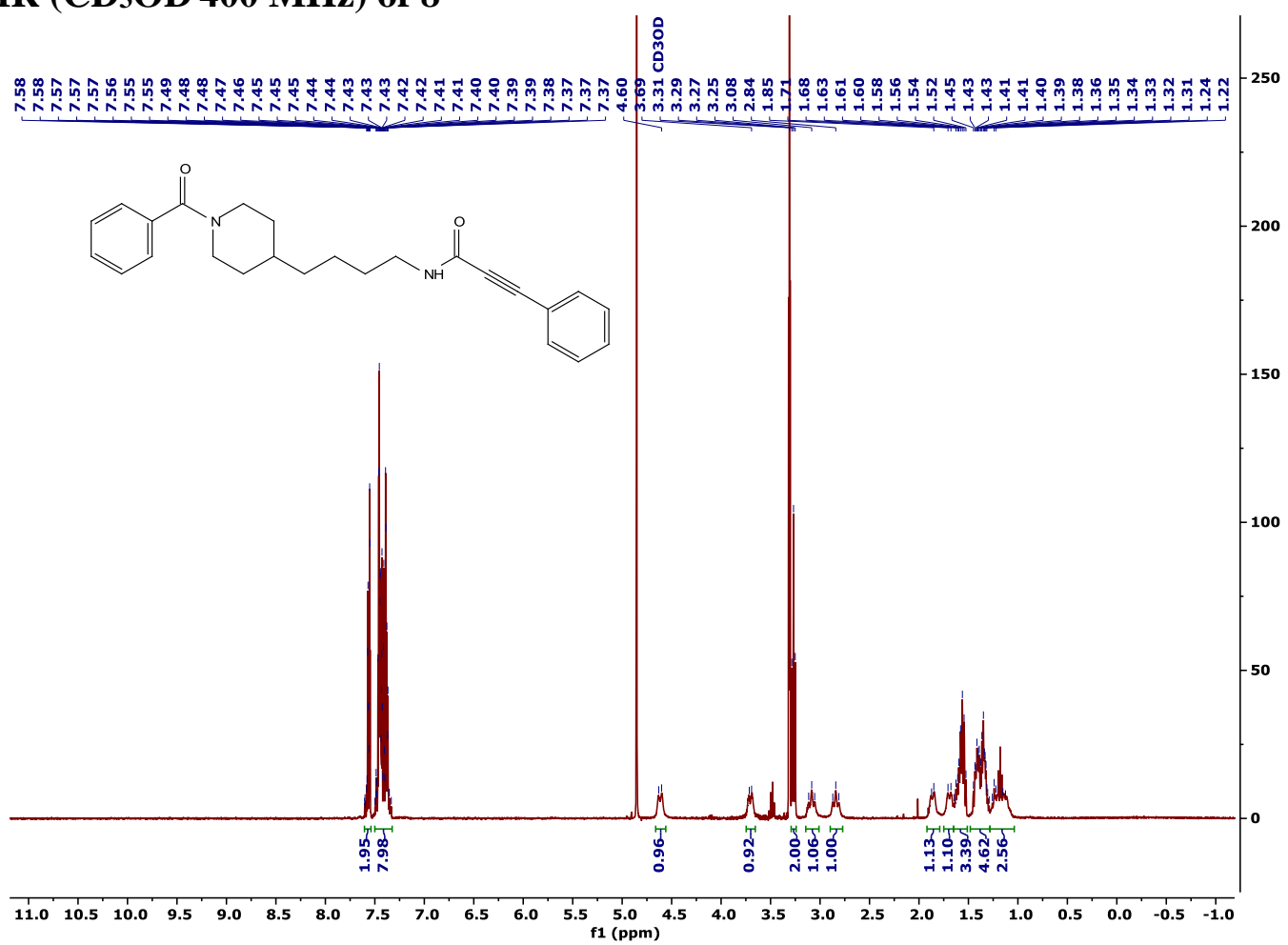

${ }^{13} \mathrm{C}$ NMR (CD 3 OD $\left.100 \mathrm{MHz}\right)$ of 8

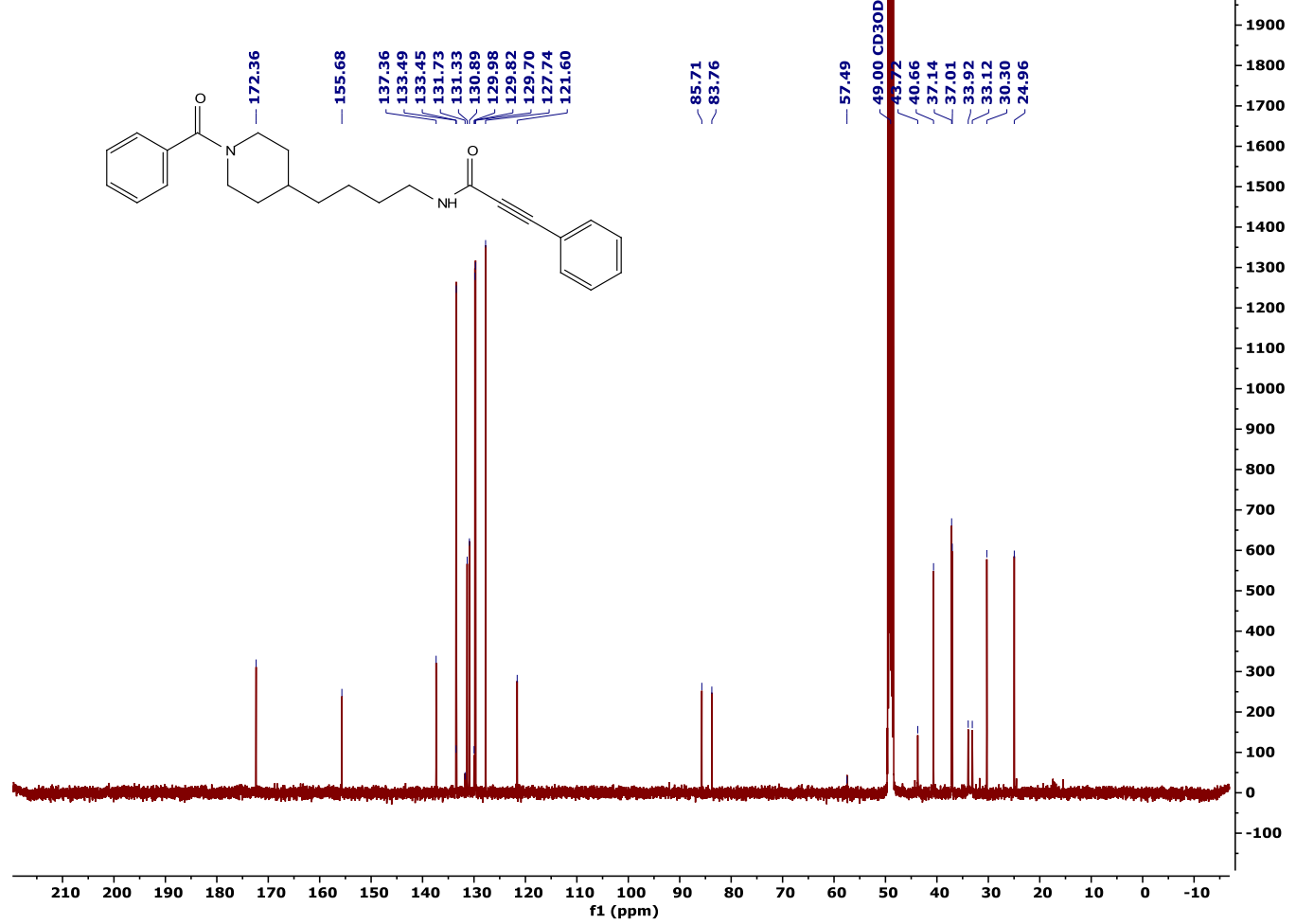


${ }^{1} \mathrm{H}$ NMR (CDCl $\left.400 \mathrm{MHz}\right)$ of 9

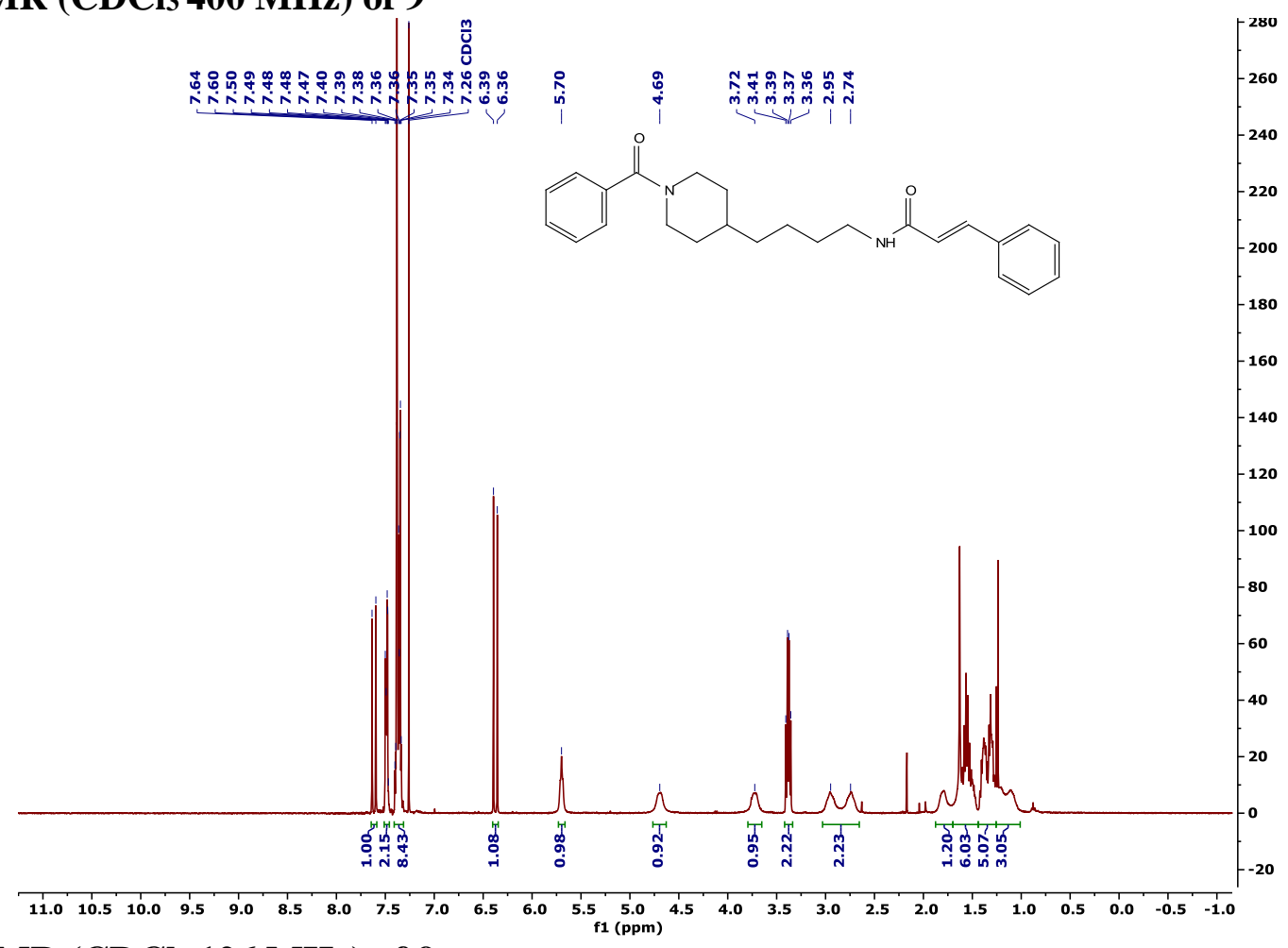

${ }^{13} \mathrm{C}$ NMR $\left(\mathrm{CDCl}_{3} 126 \mathrm{MHz}\right)$ of 9

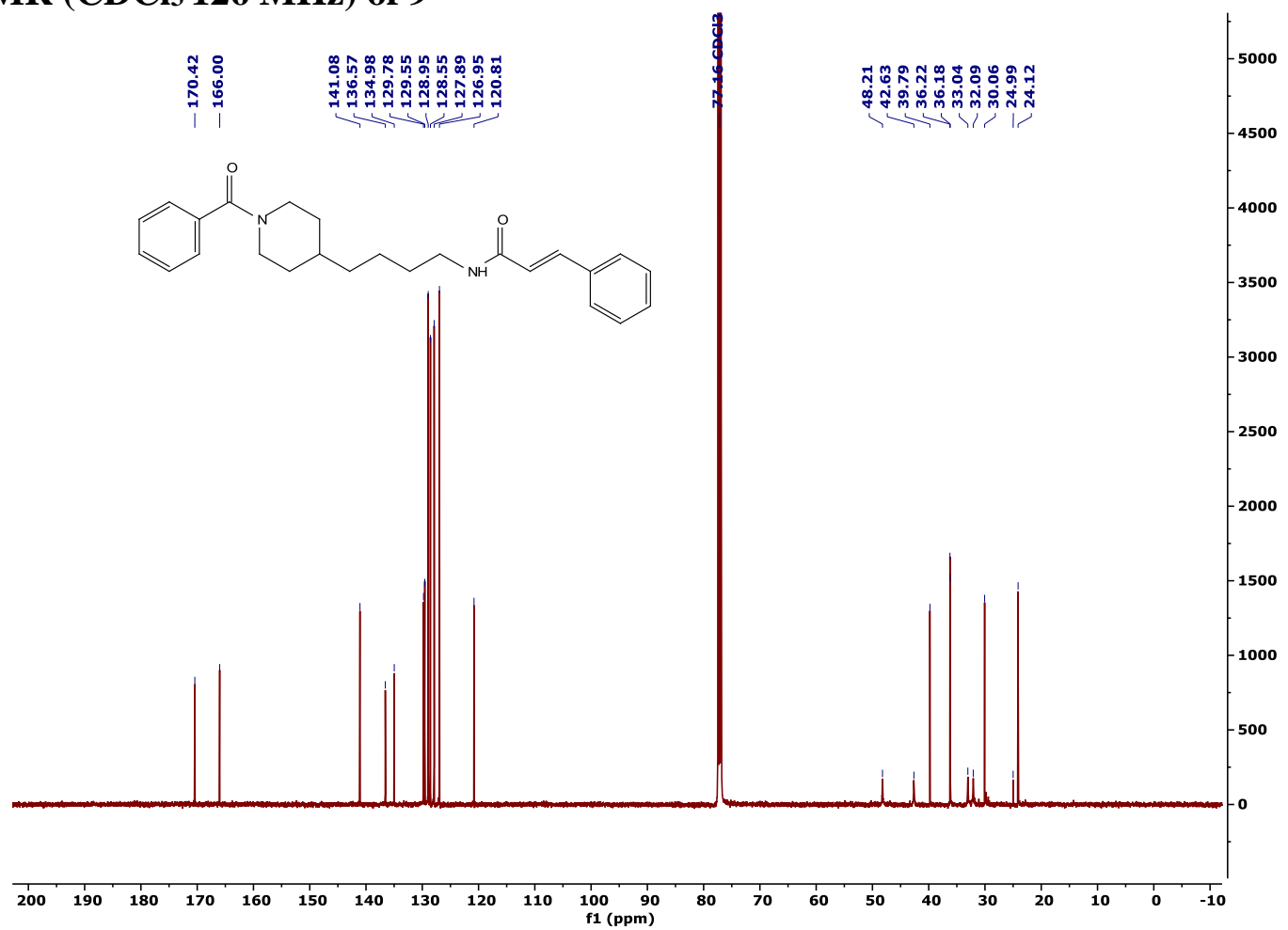


14. NMR Spectra of 11

${ }^{1} \mathrm{H}$ NMR $\left(\mathrm{CDCl}_{3} 400 \mathrm{MHz}\right)$ of $N$-deuterated 1a

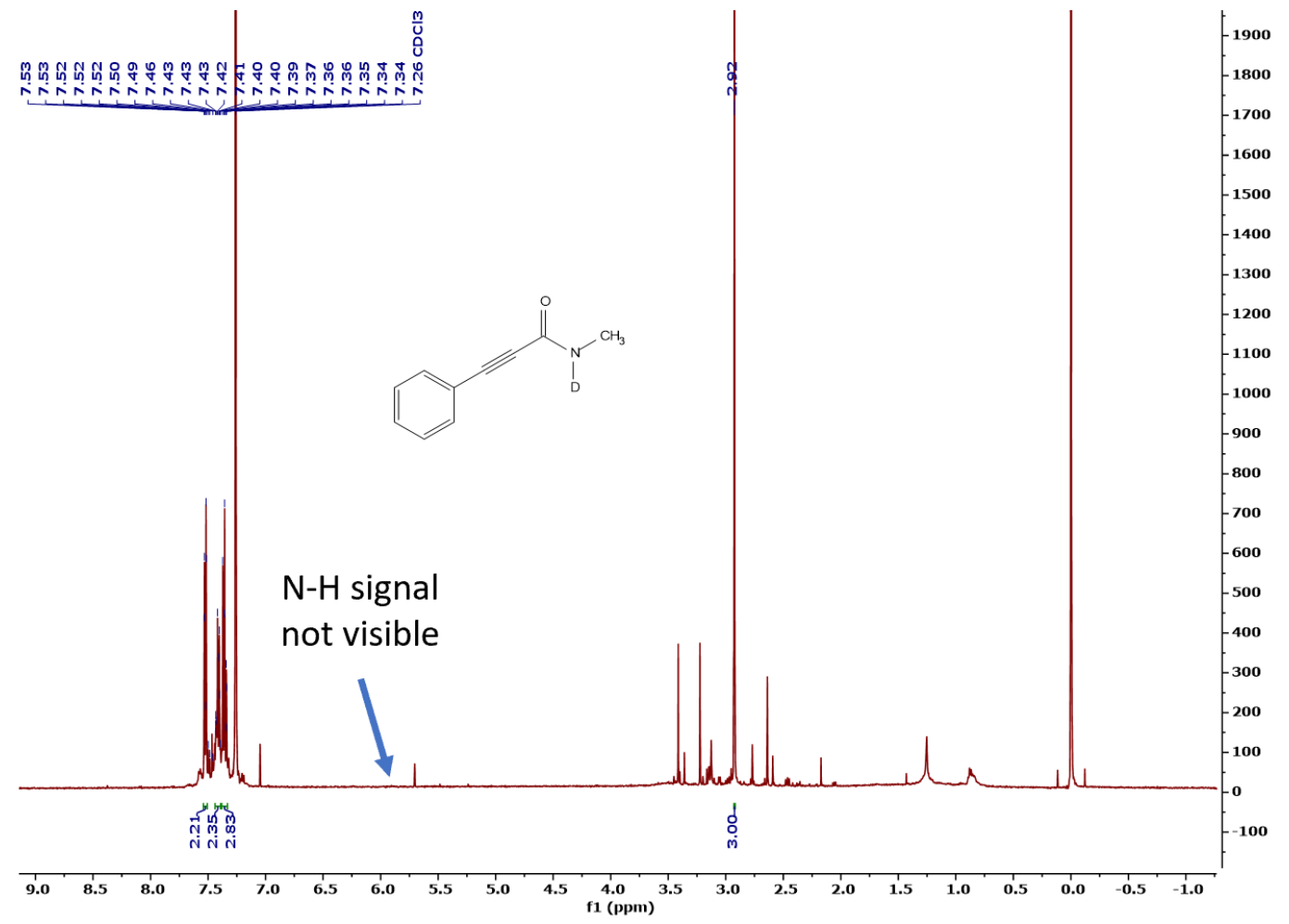

${ }^{1} \mathrm{H}$ NMR $\left(\mathrm{CDCl}_{3} 400 \mathrm{MHz}\right)$ of crude reaction mixture

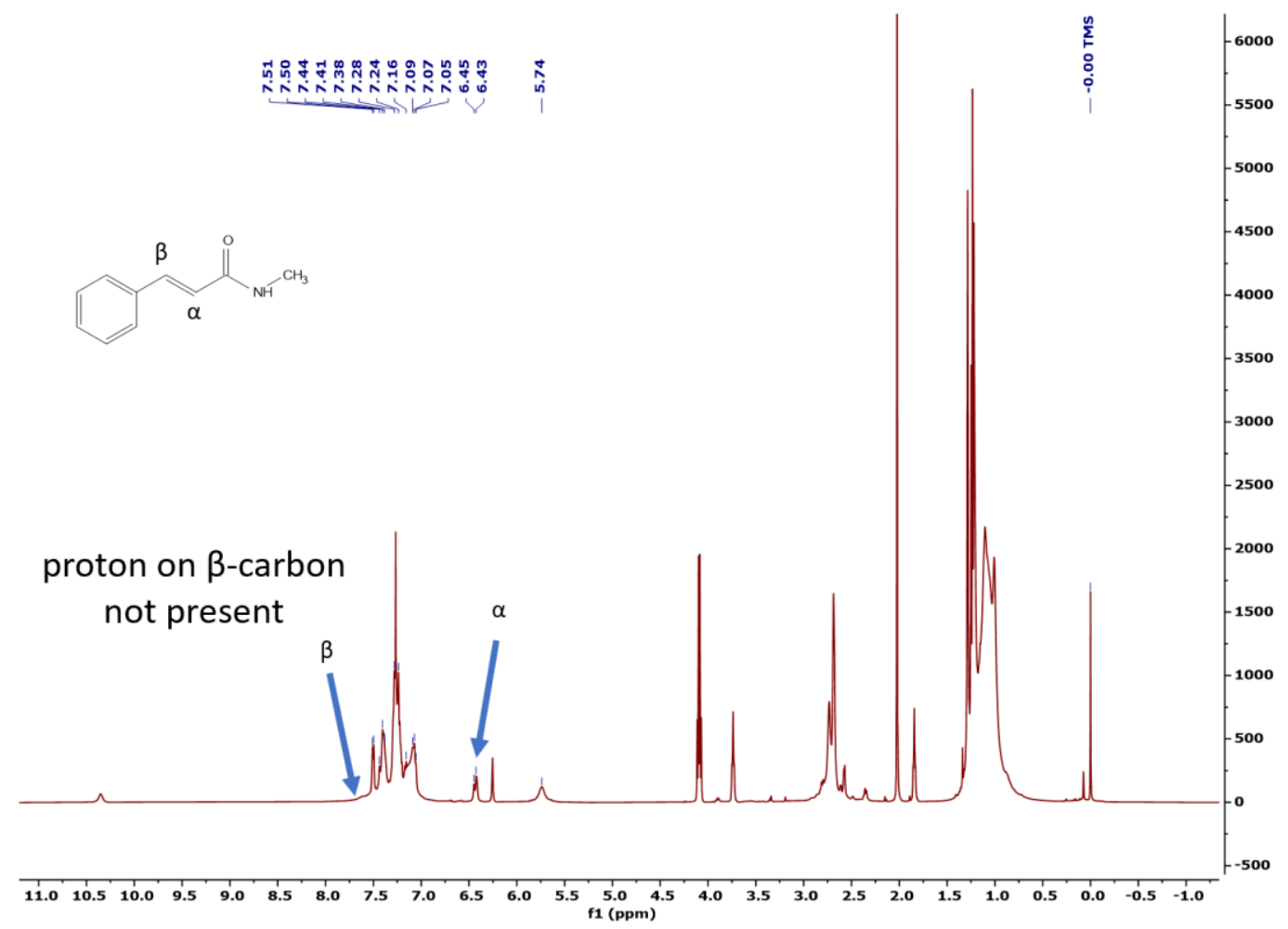


${ }^{1} \mathrm{H}$ NMR $\left(\mathrm{CDCl}_{3} 400 \mathrm{MHz}\right)$ of 11

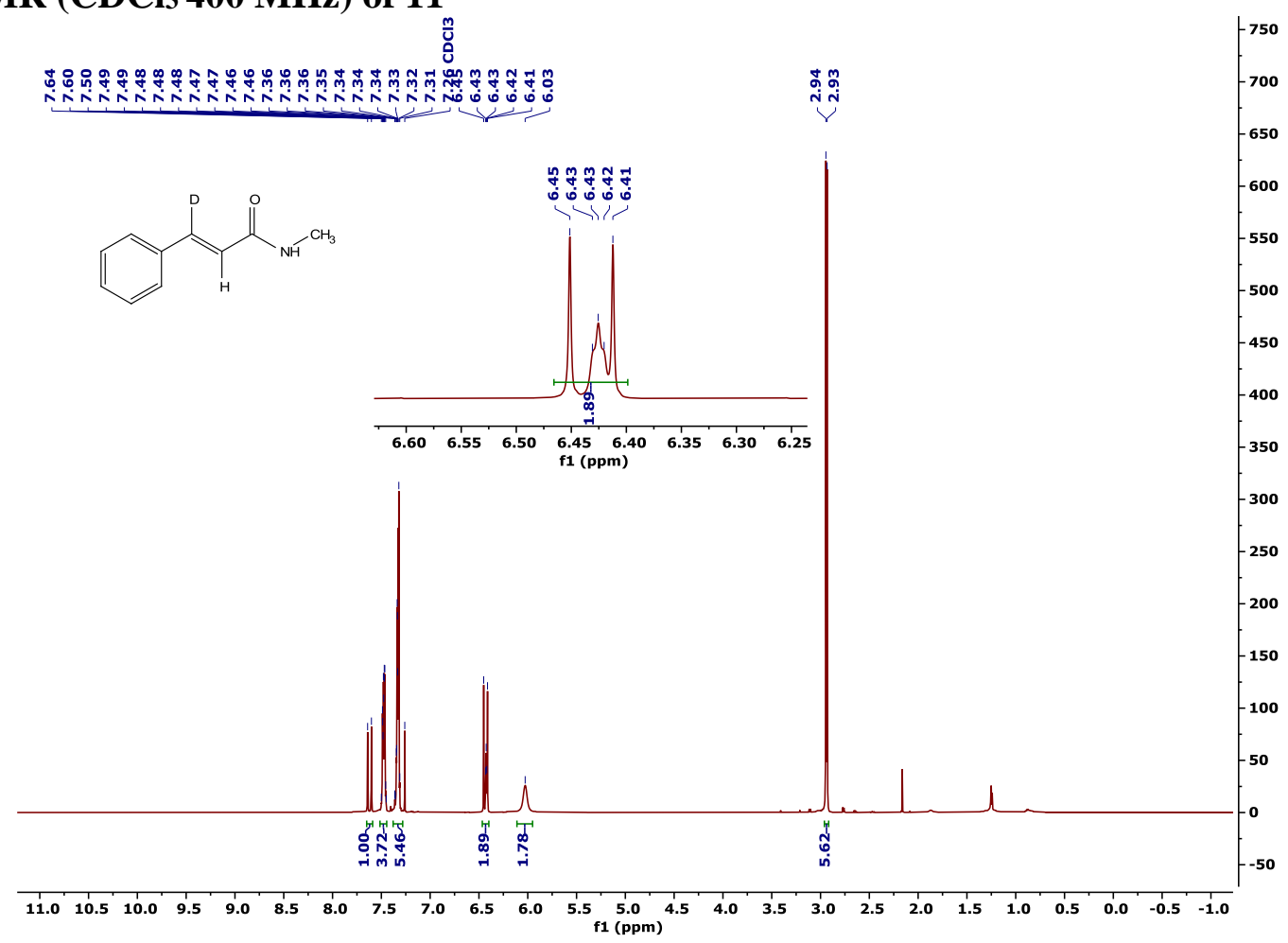

${ }^{13} \mathrm{C}$ NMR (CDCl $\left.3100 \mathrm{MHz}\right)$ of 11

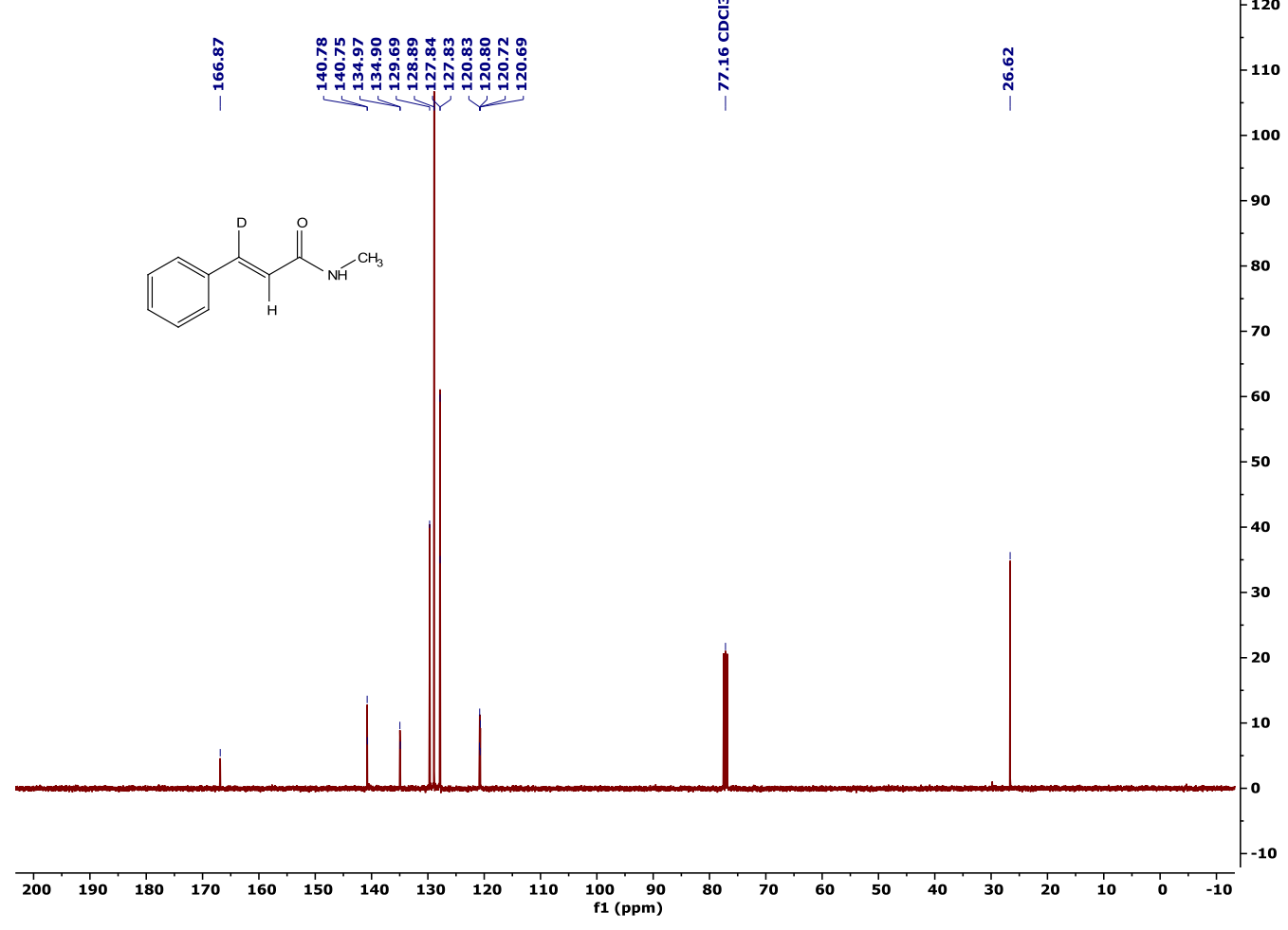


15. NMR Spectra of 12

${ }^{1} \mathrm{H}$ NMR $\left(\mathrm{CDCl}_{3} 400 \mathrm{MHz}\right)$ of 12

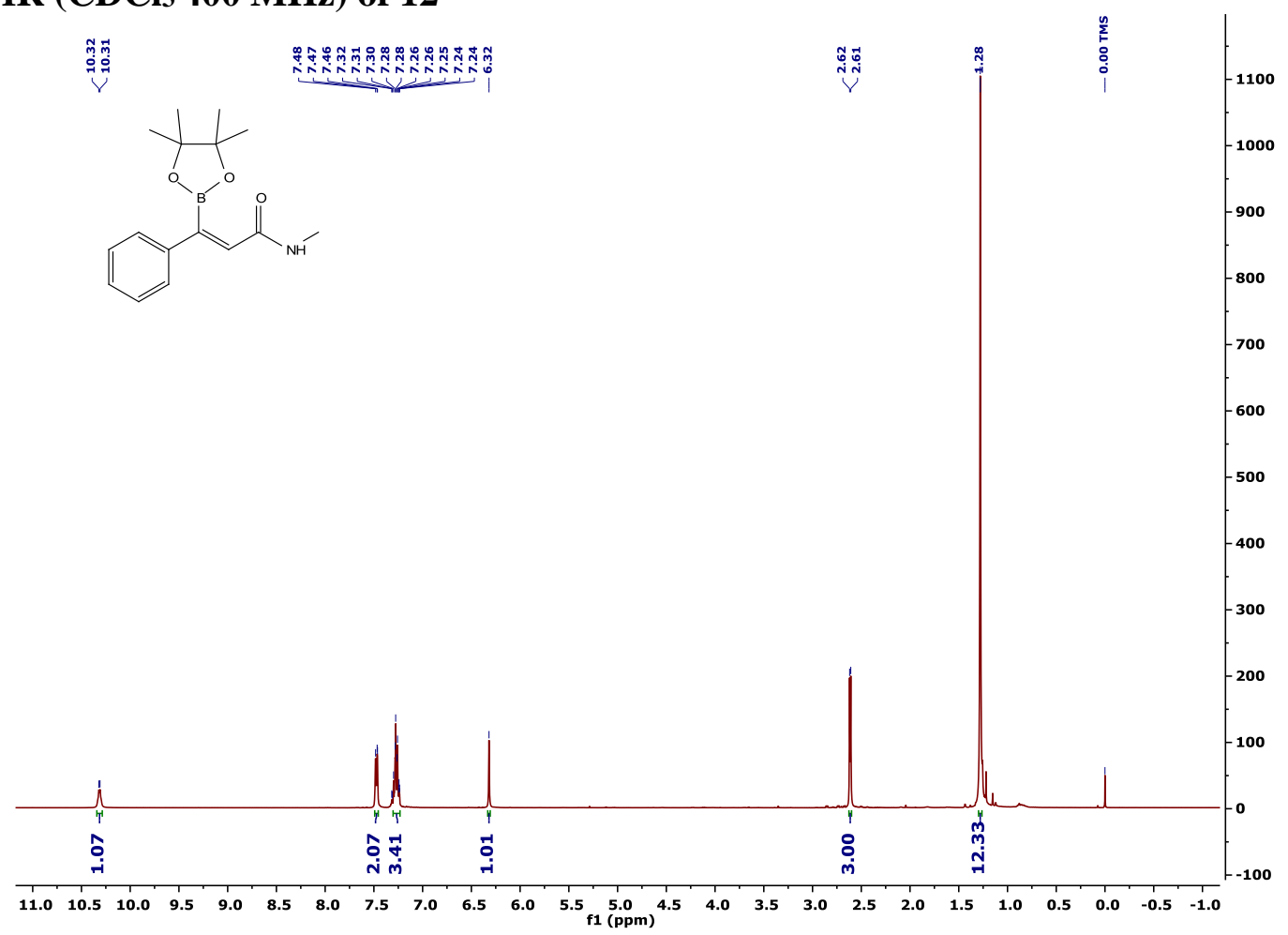

${ }^{13} \mathrm{C} \mathrm{NMR}\left(\mathrm{CDCl}_{3} 100 \mathrm{MHz}\right)$ of 12

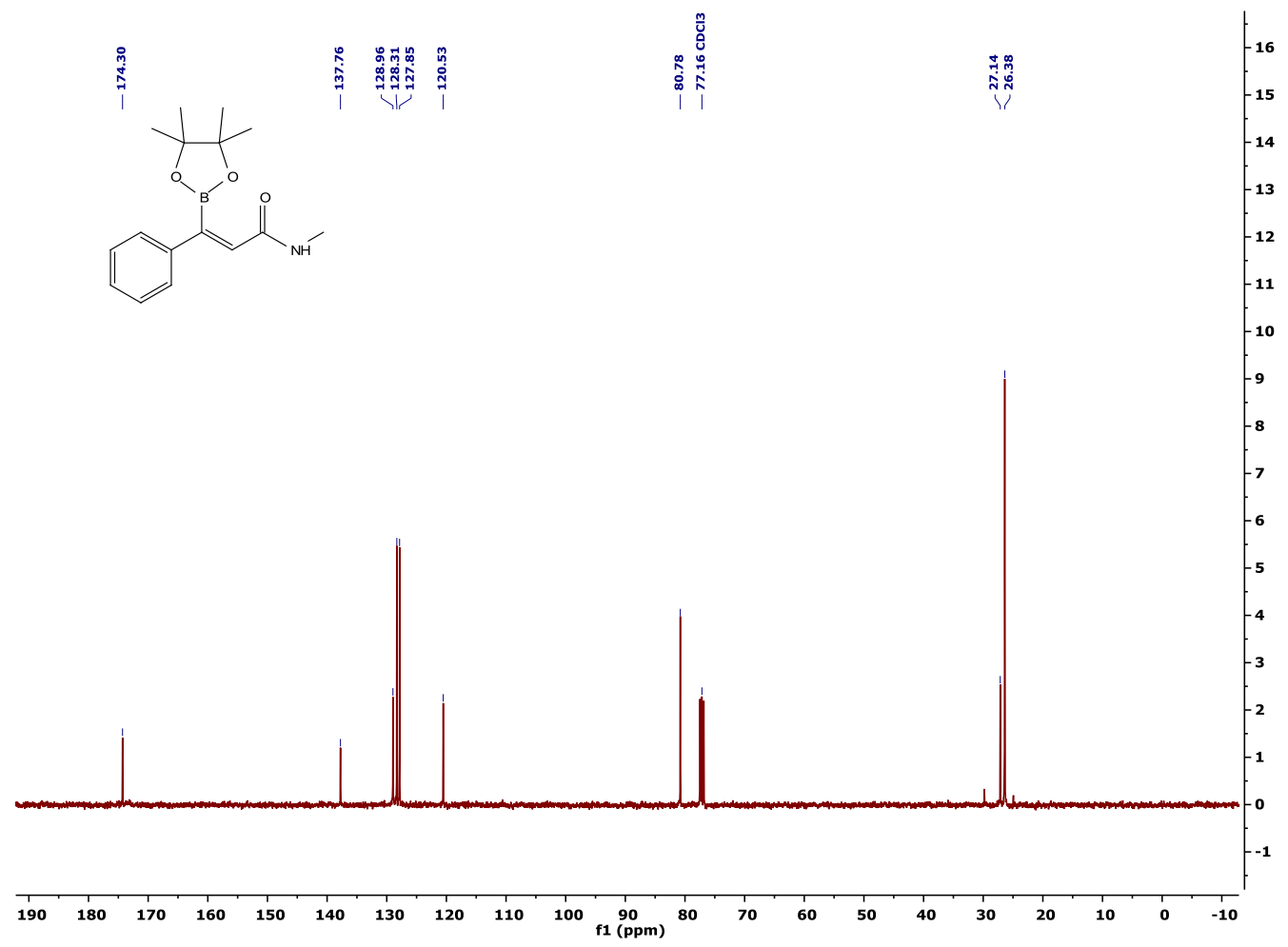




\section{References:}

1. K. Park, J.-M. You, S. Jeon, S. Lee, Eur. J. Org. Chem. 2013, 1973-1978.

2. T. Rankin, R. R. Tykwinski, Org. Lett. 2003, 5, 213-216.

3. J. Alfaro-Lopez, T. Okayama, K. Hosohata, P. Davis, F. Porreca, H. I. Yamamura, V. J. Hruby J. Med. Chem. 1999, 42, 5359-5368.

4. U. Galli, E. Ercolano, L. Carraro, C. R. B. Roman, G. Sorba, P. L. Canonico, A. A. Genazzani, G. C. Tron, R. A. Billington ChemMedChem 2008, 3, 771-779.

5. R. Justin Grams, Russell G. Fritzemeier, Carla D. Slebodnick, Webster L. Santos Org. Lett. 2019, 21, 6795-6799. 